FINAL REPORT

FHWA/IN/JTRP-2005/24

\title{
VALIDATION OF SUPERPAVE MIXTURE DESIGN AND ANALYSIS PROCEDURES USING THE NCAT TEST TRACK
}

\author{
by \\ Sivaranjan Sivasubramaniam \\ Graduate Research Assistant \\ John E. Haddock \\ Associate Professor \\ Joint Transportation Research Program \\ Project Number: C-36-56H \\ File Number: 2-13-8 \\ SPR-2199

\begin{abstract}
Conducted in Cooperation with the
And the

U.S. Department of Transportation

Federal Highway Administration
\end{abstract} \\ Indiana Department of Transportation
}

The content of this report reflect the views of authors who are responsible for the facts and accuracy of the data presented herein. The contents do not necessarily reflect the official views or policies of the Indiana Department of Transportation or the Federal Highway Administration at the time of publication. This report does not constitute a standard, specification, or regulation.

\author{
Purdue University \\ West Lafayette, IN 47907 \\ March 2006
}




\section{TECHNICAL Summary}

INDOT Research

Technology Transfer and Project Implementation Information

TRB Subject Code:31-5 Bituminous Characteristics and Tests

March 2006

Publication No.: FHWA/IN/JTRP-2005/24, SPR-2199

Final Report

\section{Validation of Superpave Mixture Design and Analysis Procedures Using the NCA Test Track}

\section{Introduction}

The Indiana Department of Transportation (INDOT) adopted the Superpave hot-mix asphalt (HMA) mixture design method and specifications during the 1990's and although long-term performance benefits were expected, long term data were not available. The INDOT therefore decided to participate in the full-scale test track experiment conducted at the National Center for Asphalt Technology (NCAT) in Alabama as a way to validate the Superpave design method and specifications. Additionally, INDOT was interested in establishing rutting performance relationships between the Accelerated Pavement Tester (APT), the Purdue Laboratory Wheel Tracker (PURWheel), and the NCAT test track (in-service) pavement sections.

The objectives of the project were fulfilled using a coordinated effort between the INDOT, the Alabama Department of Transportation (ALDOT), and the Federal Highway Administration (FHWA). Ten HMA mixtures were placed on the NCAT test track that included three binder types (one neat (unmodified) and two modified), two gradations (fine- and coarse-graded), and two binder contents (optimum and optimum plus one-half percent). Ten million Equivalent Single Axle Loads (ESAL) were applied to these test sections over a twoyear period. In addition, eight of the ten track mixtures were duplicated in the APT using the same materials as used for the track mixtures. Slab specimens were cut from both the APT and the test track before the application of traffic and these were tested in the PURWheel.

The volumetric properties of each of the ten mixtures was computed and statistically related to HMA rutting performance at the test track. Efforts to establish rutting performance relationships among the APT, PURWheel, and NCAT test track consisted of two approaches. The first directly related HMA mixture rutting performance from the PURWheel, APT, and test track. The second used finite element modeling to predict test track rutting based on PURWheel and APT test results. This latter approach first determined creep material parameters using APT and PURWheel data and used these as inputs into a finite element model of the NCAT test track. The track rutting predicted by this approach was then compared to the actual rutting experienced at the test track.

\section{Findings}

The validation portion of the study found that the Superpave mixture design method and specifications provide well-performing HMA mixtures with respect to permanent deformation. As with all HMA projects, quality materials should be used and sound construction practices followed. Additionally, the data indicate that the use of modified binders can provide higher rutting resistance than neat binders and that modified binders allow for larger binder content variation without compromising rutting performance. Finally, the fine-graded Superpave mixtures used in this study show good performance under the heavy traffic applications at the test track.

The direct rutting performance relationship between the APT and the 
PURWheel is very strong when the PURWheel specimens are taken directly from the APT test sections, but is weaker when PURWheel specimens are laboratory prepared. Likewise, there is also a fair correlation between the PURWheel and test track when PURWheel specimens are taken from the test track, but again, when laboratory prepared PURWheel specimens are used, the relationship is significantly weaker. The APT and test track also show a fair correlation in rutting performance. This correlation could be improved with better initial density control during APT test section construction.

Given the relationships established in this research, it should be possible for INDOT to place and test various HMA mixtures in the
APT and/or PURWheel and use the results to predict in-service performance of the mixtures. However, the PURWheel appears to be sensitive to the sample preparation (compaction) method and in order for the PURWheel test to better predict in-service performance, PURWheel test specimens must be prepared so as to duplicate the in-service compaction of HMA. Duplication of in-service mixtures (including initial density) in the APT also appears to be a critical element in performing accelerated testing.

Finite element modeling was used to predict test track rutting based on PURWheel and APT test results. It was concluded that the test track mixtures' rutting performance could be adequately predicted by the model.

\section{Implementation}

The research reported herein clearly demonstrates that the Superpave mixture design method and specifications do provide wellperforming HMA mixtures. INDOT should therefore fully adopt the Superpave mixture design method and specifications. Further, the INDOT should continue to monitor the performance of Superpave mixtures and make changes to the method and specifications as necessary.

Relationships among in-service pavements (as represented by the test track), APT, and
PURWheel were developed in this research and indicate good correlations between these and inservice pavements. The INDOT should establish a standard method for using the APT to test HMA mixture stability for new types of mixtures, mixture design approaches, and new mixture technologies. Also, a research program that uses the PURWheel to determine if another, more common laboratory wheel track test machine can be used for daily mixture design and field production testing should be considered.

\section{Contacts}

For more information:

Prof. John Haddock

Principal Investigator

School of Civil Engineering

Purdue University

West Lafayette IN 47907

Phone: (765) 496-3996

Fax: (765) 496-1364

E-mail: jhaddock@ecn.purdue.edu

\author{
Indiana Department of Transportation \\ Division of Research \\ 1205 Montgomery Street \\ P.O. Box 2279 \\ West Lafayette, IN 47906 \\ Phone: (765) 463-1521 \\ Fax: (765) 497-1665
}

\author{
Purdue University \\ Joint Transportation Research Program \\ School of Civil Engineering \\ West Lafayette, IN 47907-1284 \\ Phone: (765) 494-9310 \\ Fax: (765) 496-7996 \\ E:mail: jtrp@ecn.purdue.edu \\ http://www.purdue.edu/jtrp
}




\section{TABLE OF CONTENTS}

TABLE OF CONTNETS...................................................................................ii

LIST OF TABLES ...........................................................................................

LIST OF FIGURES .......................................................................................viii

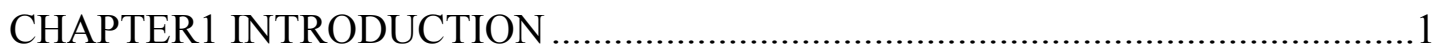

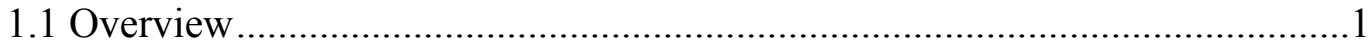

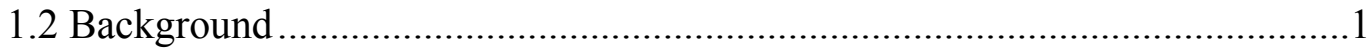

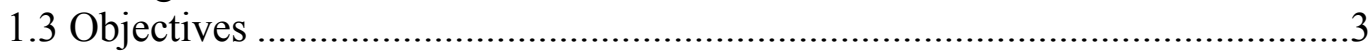

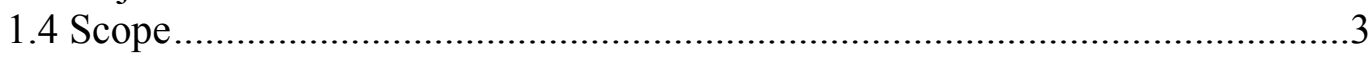

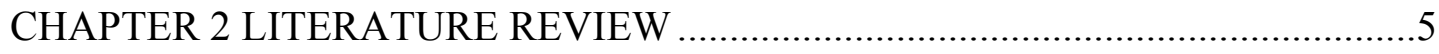

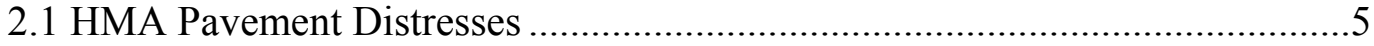

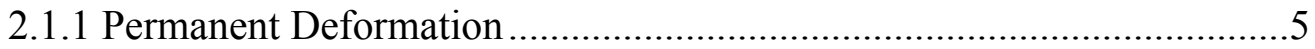

2.1.1.1 Rutting Development Mechanism ...............................................5

2.1.1.2 Definitions and Measurement of Rutting ..................................8

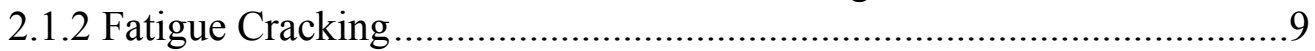

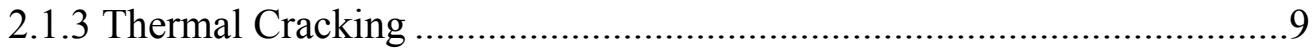

2.2 HMA Mixture Rutting Performance ............................................................ 10

2.2.1 Aggregate Properties................................................................. 10

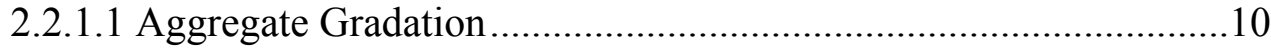

2.2.1.2 Aggregate Shape and Surface Texture...................................... 12

2.2.1.3 Dust Proportion ......................................................................... 13

2.2.1.4 Nominal Maximum Aggregate Size ......................................... 13

2.2.2 Binder Properties .................................................................... 14

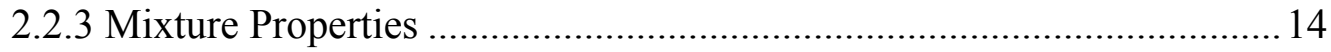

2.2.3.1 Binder Content ........................................................................ 14

2.2.3.2 Air Void Content ....................................................................... 15

2.2.3.3 Voids in Mineral Aggregate........................................................ 15

2.2.3.4 Voids Filled With Asphalt ....................................................... 16

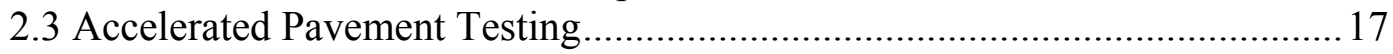

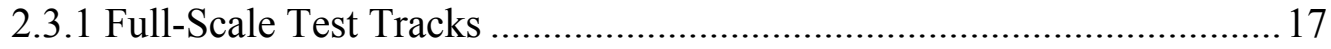


2.3.2 Full-Scale Accelerated Loading Devices............................................. 25

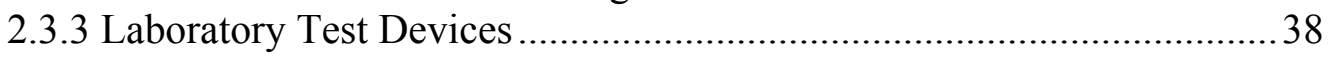

2.4 Modeling of HMA Mixtures and Pavements ............................................ 46

2.4.1 Behavior of Asphalt Binder ..............................................................46

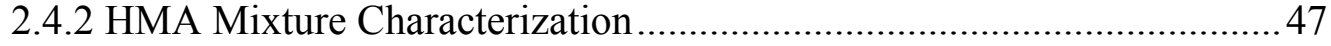

2.4.3 Modeling of HMA Rutting ......................................................... 51

CHAPTER 3 EXPERIMENTAL DESIGN AND RESEARCH APPROACH ...........61

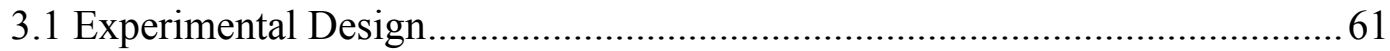

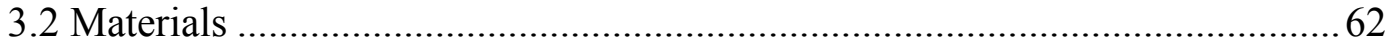

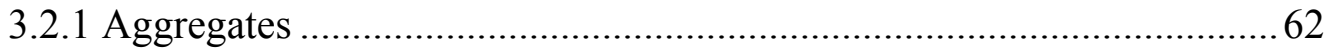

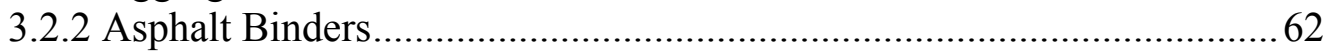

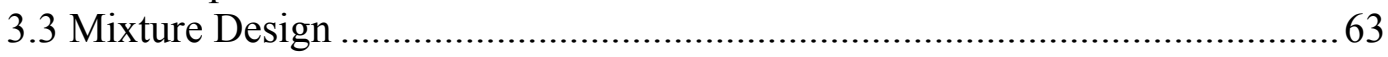

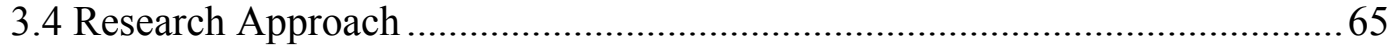

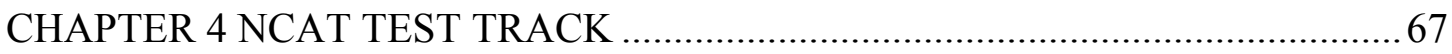

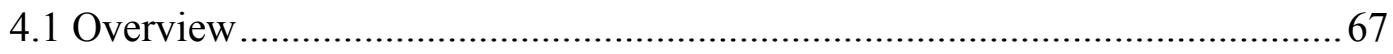

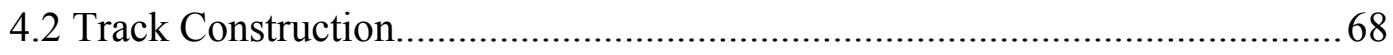

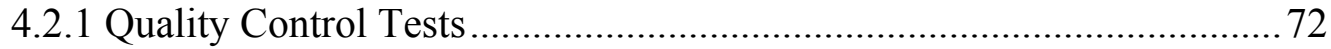

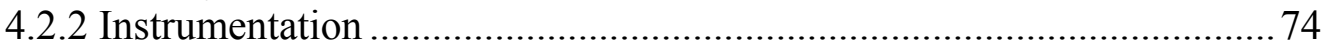

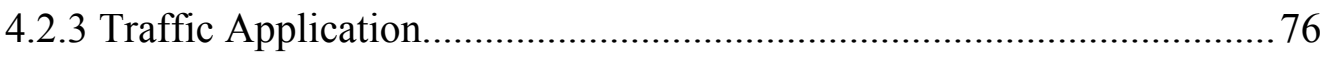

4.2.4 Performance Data Collection .......................................................... 77

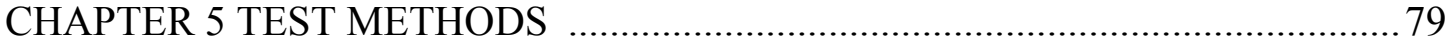

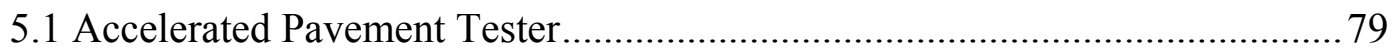

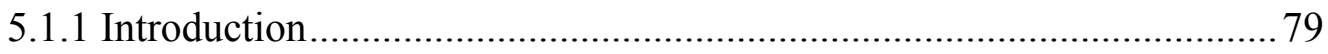

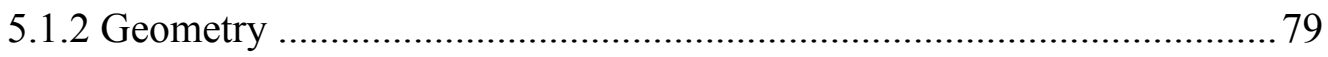

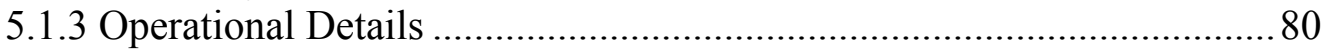

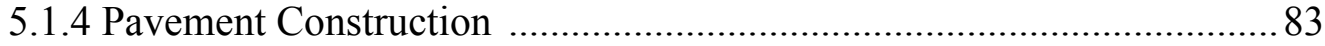

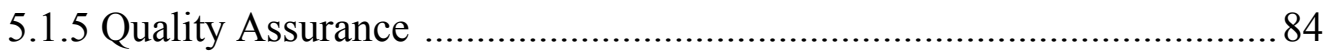

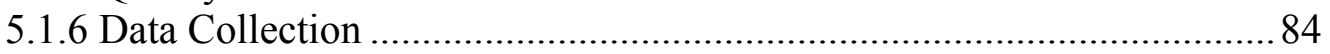

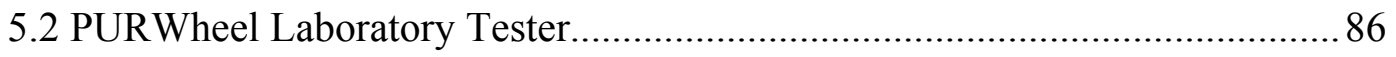

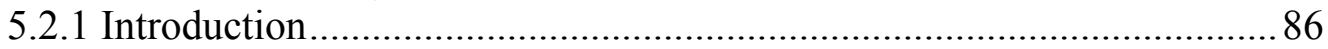

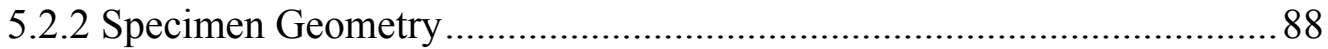

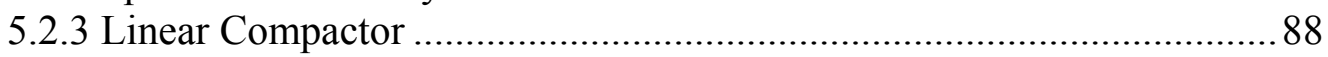

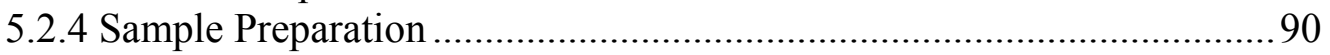

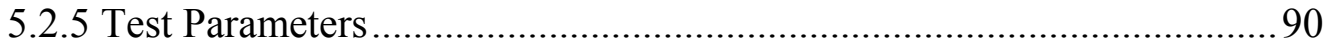

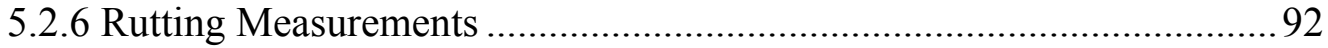

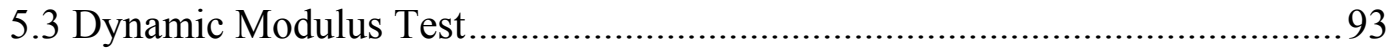

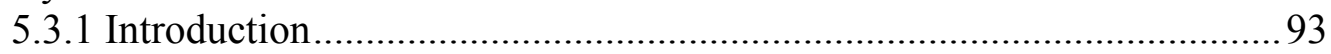

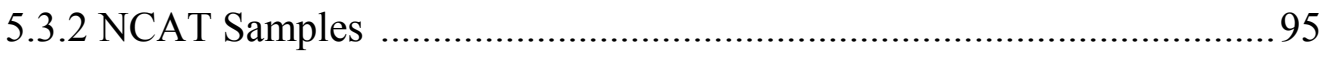

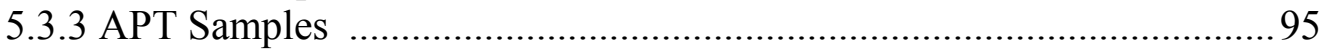


CHAPTER 6 EXPERIMENT TEST RESULTS AND DATA ANALYSIS .............97

6.1 Validation of Superpave Mixture Design Method .......................................... 97

6.1.1 Superpave Binder Requirements.......................................................97

6.1.2 Superpave Volumetric Requirements ............................................ 98

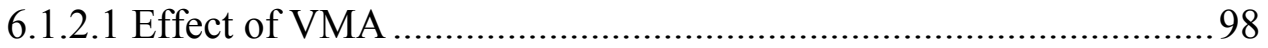

6.1.2.1 Effect of VMA ...................................................................... 100

6.1.2.3 Effect of Dust Proportion...................................................... 100

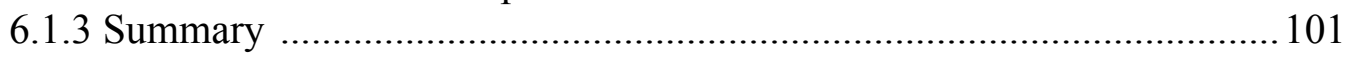

6.2 Effect of As-constructed Volumetrics on Rutting Performance 102

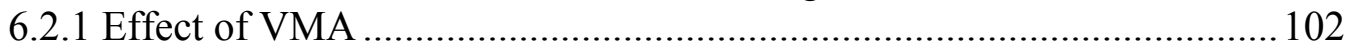

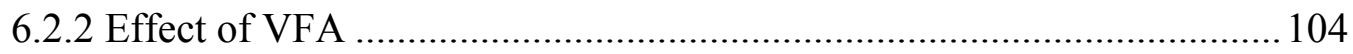

6.2.3 Effect of Dust Proportion................................................................... 105

6.2.4 Effect of Binder Film Thickness ........................................................ 106

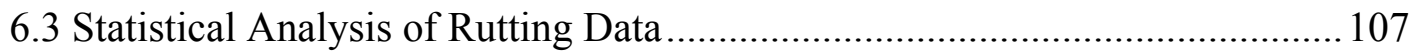

6.4 Effect of Mixture Properties on Rutting Performance .................................... 109

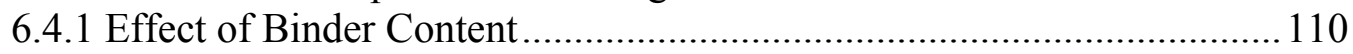

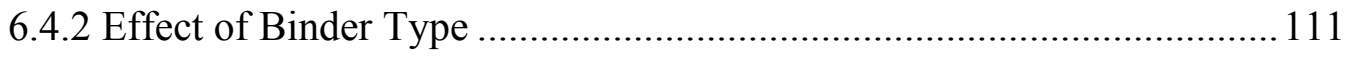

6.4.3 Effect of Aggregate Gradation...................................................... 113

6.4.4 Effect of Initial Density ................................................................ 114

6.5 Effect of Compaction Method on Rutting Performance ................................. 118

6.6 Correlation of Direct Rutting Performance................................................... 119

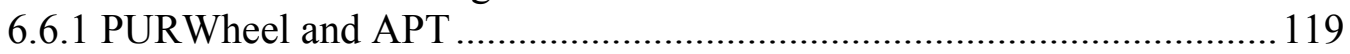

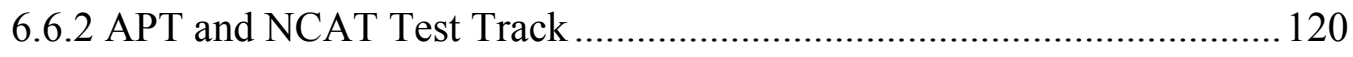

6.6.3 PURWheel and NCAT Test Track .................................................. 121

6.6.4 Dynamic Modulus Test and NCAT Track.......................................... 123

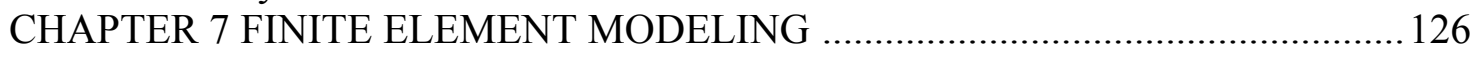

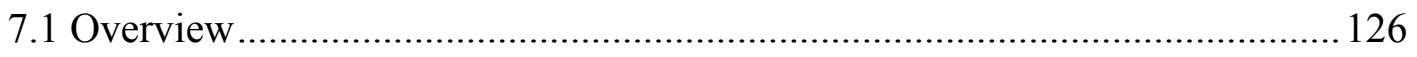

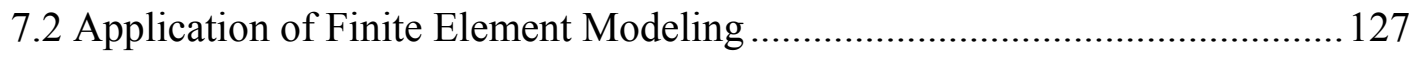

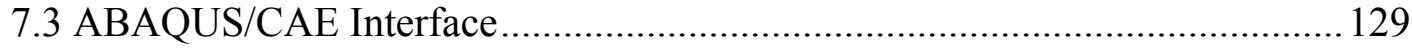

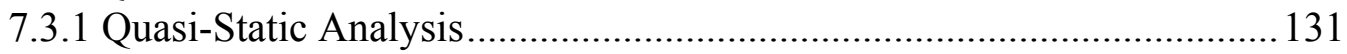

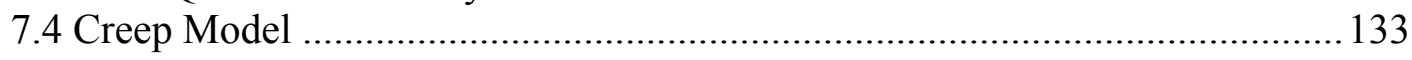

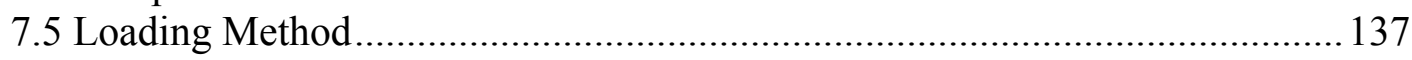

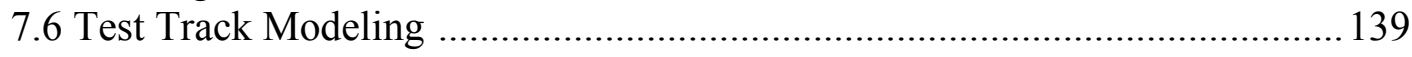

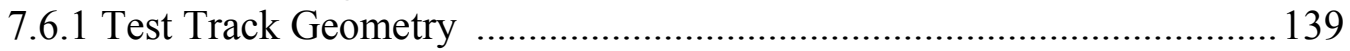

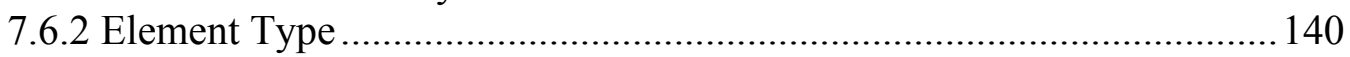

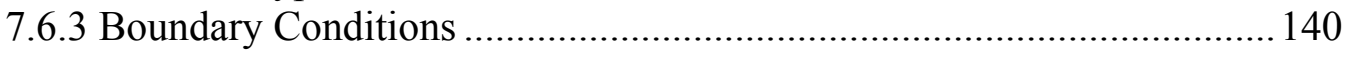

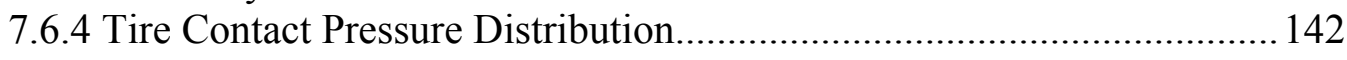

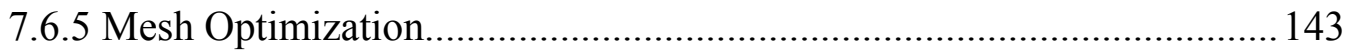

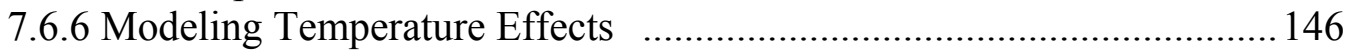

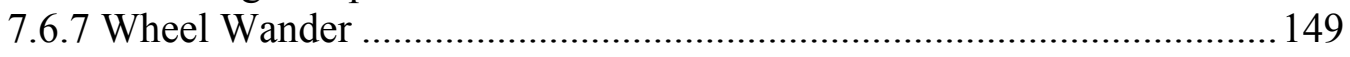




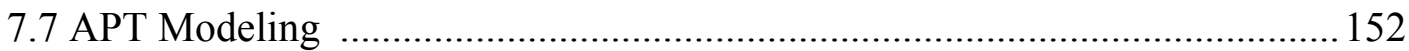

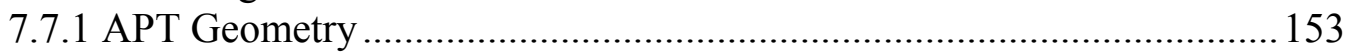

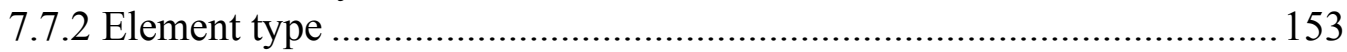

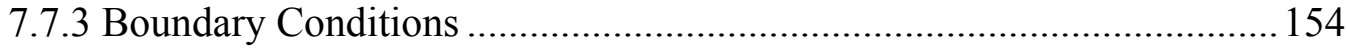

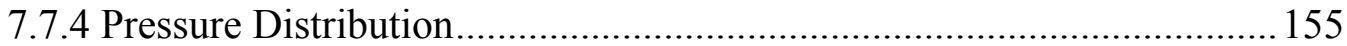

7.7.4.1 Vehicle-Road Surface Pressure Transducer Array (VRSPTA) .....156

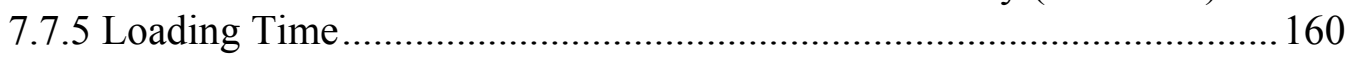

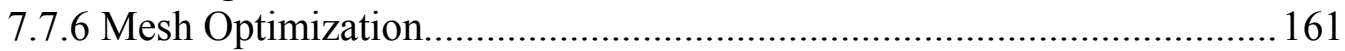

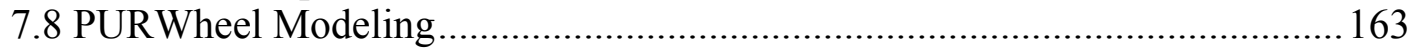

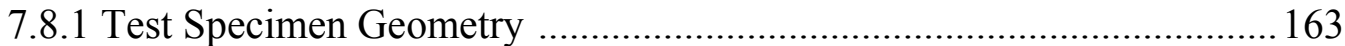

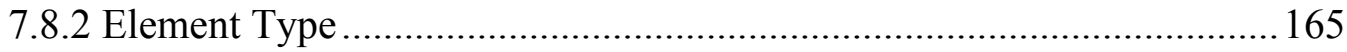

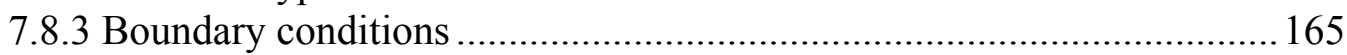

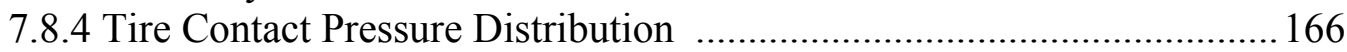

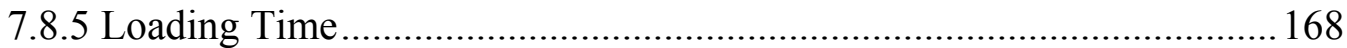

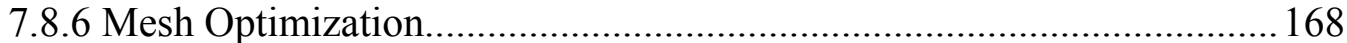

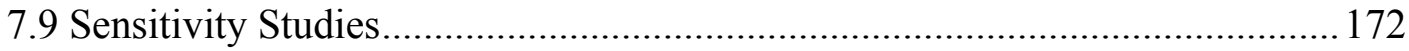

7.9.1 Effect of Creep Model Parameters.......................................................... 172

7.10 Determination of creep material parameters and Correlation Relationships. 176

7.10.1 Determination of NCAT Test Track Creep Parameters......................... 177

7.10.2 Determination of APT Creep Parameters .............................................. 179

7.10.3 Determination of PURWheel Creep Parameters...................................... 181

7.10.4 Rutting Relationships using Determined Creep Parameters .................. 184

7.10.5 Densification Effects on the Rutting Performance ……………............ 185

7.11 Temperature Effects on Rutting Performance (FEM) .................................... 187

CHAPTER 8 CONCLUSIONS AND RECOMMENDATIONS ………....................192

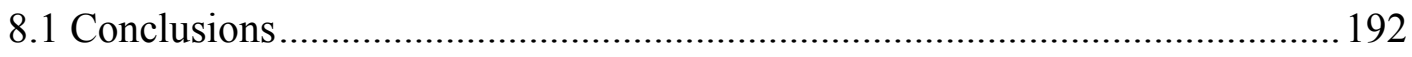

8.1.1 Superpave Mixture Design Method Validation ........................................ 193

8.1.2 Establishment of Rutting Performance Relationships ............................. 193

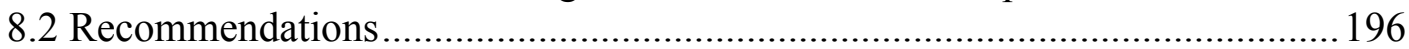

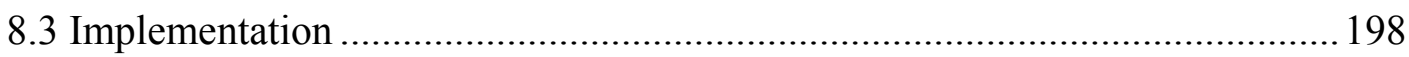

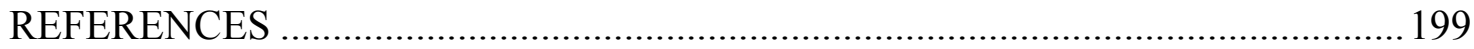

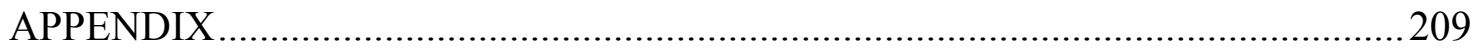




\section{LIST OF TABLES}

Table

Table 2-1 Loading of AASHO Road Test (After Huang, 1993).............................. 18

Table 2-2 Experimental Design of the Original WestTrack Sections (After Epps et al., 1998).

Table 2-3 WesTrack Rut Depth Summary (After Hand et al., 1998)

Table 2-4 FHWA ALF Superpave Binder Validation Study Design (Bonaquist et al., 1997) 29

Table 2-5 Asphalt Rutting Performance (After Bonaquist et al., 1997) ..................... 30

Table 2-6 National Pooled Funds No. 176 Experimental Design (After Stiady, 2000)

Table 2-7 Factor Levels of the SHRP Binder Validation Study (Leahy et al., 1994) . 39

Table 2-8 SHRP Binder Validation Study ANOVA Results (Leahy et al., 1994) ......40

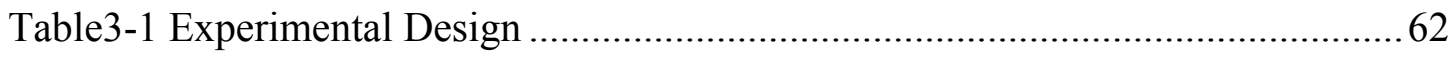

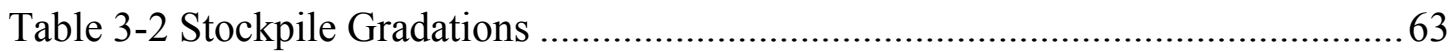

Table 3-3 Details of the mixture type, stockpile, and bin percentages ...................... 64

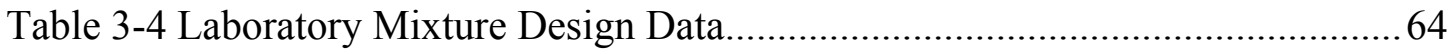

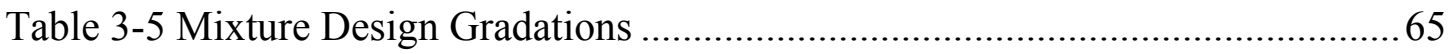

Table 4-1 As Constructed NCAT Test Track Mixture Properties .............................. 74

Table 5-1 Advantages and Disadvantages of the Triaxial Dynamic Modulus Test (Witzack et al., 2002). 
Table 6-2 NCAT Track As-Constructed Mixture Properties and Rutting Data 102

Table 6-3 APT As-Constructed Mixture Properties and Rutting Data..... 103

Table 6-4 Statistical Analysis of Total Rut Data ................................................ 108

Table 6-5 Dynamic Modulus Test Data.................................................................. 124

Table 7-1 Creep Parameters of Test Track Mixtures (Region III)............................ 178

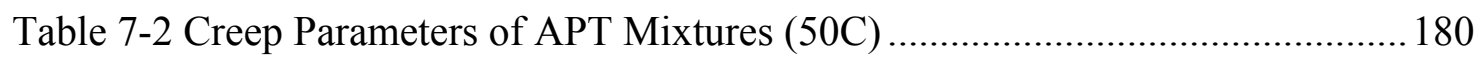

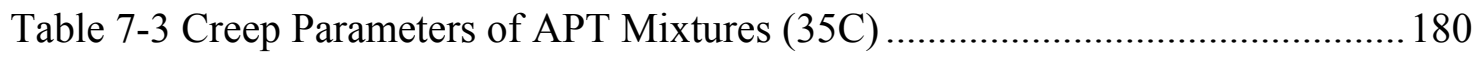

Table 7-4 Creep Parameters of PURWheel Mixtures (Track Slabs) ........................... 182

Table A-1 Creep Parameters of Test Track Mixtures (Region I) .............................208

Table A-2 Creep Parameters of Test Track Mixtures (Region II) ............................208

Table A-3 Creep Parameters of Test Track Mixtures (Region V)............................208

Table A-4 Creep Parameters of Test Track Mixtures (Region VI) ...........................209

Table A-5 Creep Parameters of PURWheel Mixtures (APT Slabs) ..........................209 


\section{LIST OF FIGURES}

Figure

Page

Figure 2-1 Rutting Caused by Weak Subgrade (After McGinnis et al., 1995)...........6

Figure 2-2 Rutting Caused by HMA Shear Failure (After McGinnis et al., 1995) .....6

Figure 2-3 Rut Definition, No Wander (After Steiady, 2000) ................................ 8

Figure 2-4 Effect of Gradation on Rutting at WesTrack (After Hand et al, 2000)...... 11

Figure 2-5 WesTrack Layout (After Epps et al., 1998) ........................................... 21

Figure 2-6 Target Gradations of WesTrack HMA Mixtures (After Epps et al., 1998)22

Figure 2-7 Effect of $\mathrm{G}^{*} / \sin \delta$ on Rutting (After Bonaquist and Mogawer, 1997).......31

Figure 2-8 Relationship between APT Total Rut Depth and PURWheel Rut Depth

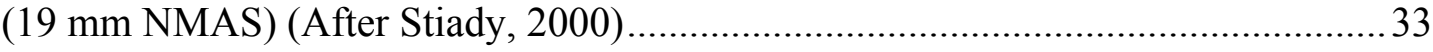

Figure 2-9 Relationship between APT Total Rut Depth and PURWheel Rut Depth

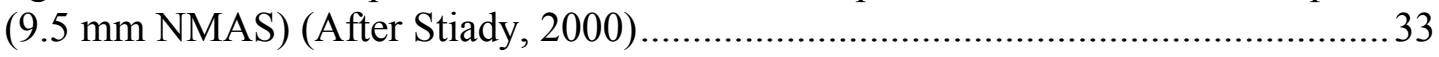

Figure 2-10 APT Rutting Performance of Conventional HMA mixtures Tested at 827 KPa Tire Pressure (After Haddock et al., 1998) ................................................ 34

Figure 2- 11 APT Rutting Performance of a $19.0 \mathrm{~mm}$ Superpave HMA mixture Testes at $827 \mathrm{KPa}$ (After Haddock et al., 1998) ................................................................ 34

Figure 2-12 Relationship between $\mathrm{G}^{*} / \sin \delta$ and Total Rut Depth (After Leahy et al.,

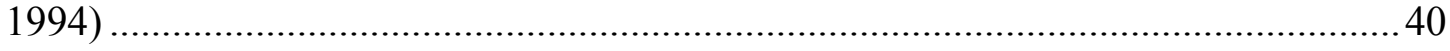

Figure 2-13 Relationship between $\mathrm{G}^{*} / \sin \delta$ and Rut Rate (Bouldin et al., 1994) ...... 41

Figure 2-14 Relationship between WesTrack Rut Depth and Number of PURWheel Passes required for $6.35 \mathrm{~mm}$ Rut Depth (After Brown et al., 2001)..... 
Figure

Figure 2-15 Relationship between Peak Strain at RSCH Test and Rut Depth in APA for Wearing Course with PG 64-22 Binder (After Kandhal et al., 1999)....

Figure 2-16 Visco-Elastic Behavior of Asphalt Binder

(After McGennis et al., 1995) 46

Figure 2-17 Predicted and Measured Surface Profiles of the Pavement Simulation Test $(\mathrm{t}=0.2 \mathrm{~s}$ and $\mathrm{T}=35 \mathrm{C}$ ) (After Seibi et al., 2000)..... 50

Figure 2-18 Relationship between Predicted Permanent Deformation by Mechanolattice (ML) and Measured Values by ALF (After Yandell and Behzadi, 1999) ........52

Figure 2-19 Relationship between predicted permanent deformation by VESYS and measured values by ALF (After Yandell and Behzadi, 1999) 53

Figure 2-20 Relationship between Predicted Permanent Deformation by Modified CIRCLY and Measured Values by ALF (After Yandell and Behzadi, 1999) 53

Figure 2-21 Predicted and Measured Rut Depth Development (After Huang et al., 2000) 55

Figure 2-22 Fitted Curve of Single Wheel Path Rutting for 39 Below Mixture, Low Density (After Hua, 2000) 57

Figure 4-1 Test Track Layout (After Powell, 2001) 68

Figure 4-2 Pavement Layer Structure at NCAT Test Track (After Powell, 2001)......69

Figure 4-3 Double Barrel On-site Drum Plant (After Hand et al., 2000) .70

Figure 4-4 Typical Paving Operation (After Hand et al., 2000) ............................... 72

Figure 4-5 RTSD Sampling (After Hand et al., 2000)........................................... 73

Figure 4-6 Temperature Probe Locations (After Brown et al., 2002) ....................... 75

Figure 4-7 Truck Configuration (NCAT Website) ................................................ 77

Figure 4-8 Transverse Profile Data Collection-Using Dipstick (After Powell, 2002) 78

Figure 5- 1 Typical APT Cross Section 80 
$\begin{array}{lll}\text { Figure } & \text { Page }\end{array}$

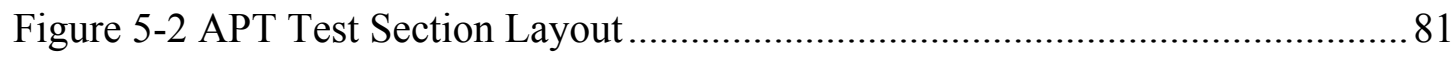

Figure 5-3 Accelerated Pavement Tester Schematic (After Hua, 2000) ..................... 82

Figure 5-4 APT Loading Configuration (After Hua, 2000)......................................... 83

Figure 5-5 APT Profile Data Collection (After Hua, 2000) ………………………..... 85

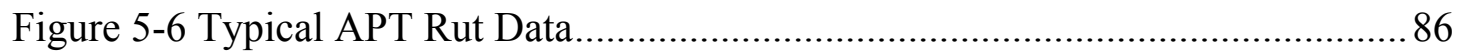

Figure 5-7 PURWheel Testing Device .................................................................. 88

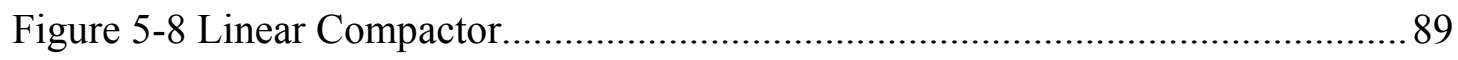

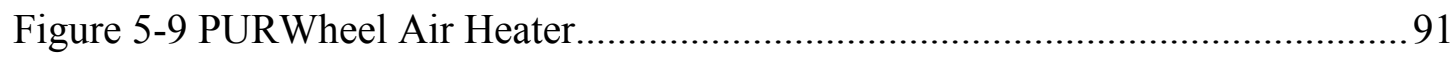

Figure 5-10 PURWheel Linear Transducer …………………………...................... 92

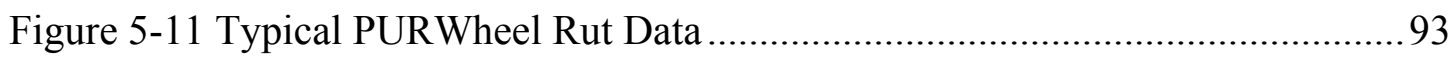

Figure 5-12 Dynamic Modulus Haversine Loading (After Witzack et al., 2002) .......94

Figure 5-13 Dynamic Modulus Test Configuration (After Witzack et al., 2002) .......95

Figure 6-1 Effect of G*/sin $\delta$ on Rutting Performance (NCAT Track).......................98

Figure 6-2 Effect of $\mathrm{G}^{*} / \sin \delta$ on Rutting Performance (APT) …………………....... 98

Figure 6-3 Effect of Design VMA on Rutting Performance (NCAT Track)...............99

Figure 6-4 Effect of Design VFA on Rutting Performance (NCAT Track) ................ 100

Figure 6-5 Effect of Design Dust Proportion on Rutting Performance

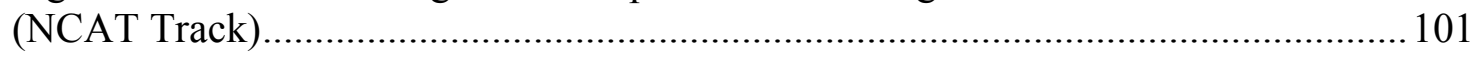

Figure 6-6 Effect of As-Constructed VMA on Rutting Performance (NCAT Track). 103

Figure 6-7 Effect of As-Constructed VMA on Rutting Performance (APT) .............. 104

Figure 6-8 Effect of As-Constructed VFA on Rutting Performance (NCAT Track) .. 104

Figure 6-9 Effect of As-Constructed VFA on Rutting Performance (APT) ................. 105 
Figure $\quad$ Page

Figure 6-10 Effect of Dust Proportion on Rutting Performance (NCAT Track)........ 105

Figure 6-11Effect of Dust Proportion on Rutting Performance (APT) ...................... 106

Figure 6-12 Effect of Asphalt Film Thickness on Rutting Performance (NCAT) ...... 107

Figure 6-13 Effect of Asphalt Film Thickness on Rutting Performance (APT).......... 107

Figure 6-14 Effect of Binder Content on NCAT Track Rutting Performance ............ 110

Figure 6-15 Effect of Binder Content on APT Rutting Performance ........................ 111

Figure 6-16 Effect of Binder Type on NCAT Track Rutting Performance................. 112

Figure 6-17 Effect of Binder Type on APT Rutting Performance.............................. 112

Figure 6-18 Effect of Aggregate Gradation on NCAT Track Rutting Performance ... 113

Figure 6-19 Effect of Aggregate Gradation on APT Rutting Performance ................. 114

Figure 6-20 Relationship between Initial density and Rutting for the Test Track.......115

Figure 6-21 Relationship between Initial density and Rutting for the APT ............... 116

Figure 6-22 Relationship between Initial density and Rutting for the PURWheel

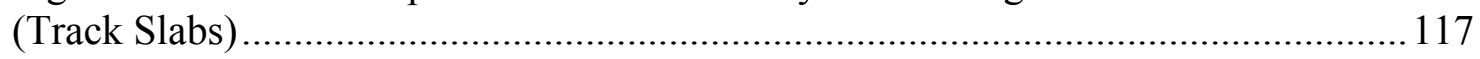

Figure 6-23 Relationship between Initial density and Rutting for the PURWheel

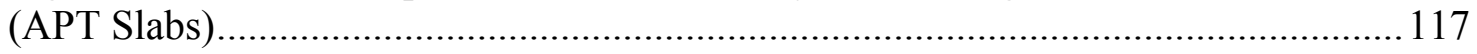

Figure 6-24 Relationship between Test Track and Linear Compaction ..................... 118

Figure 6-25 Comparison of APT and PURWheel Rutting .................................... 119

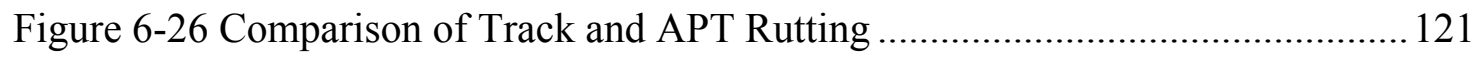

Figure 6-27 Comparison of Track and PURWheel Rutting................................... 122

Figure 6-28 Comparison of Track and PURWheel Rutting................................... 123

Figure 6-29 Relationship between Dynamic Modulus and Track Rut Depth (Track

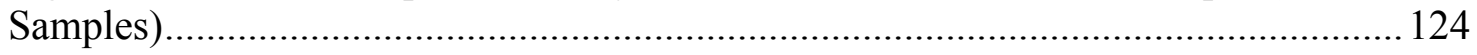


Figure $\quad$ Page

Figure 6-30 Relationship between Dynamic Modulus and Track Rut Depth (APT

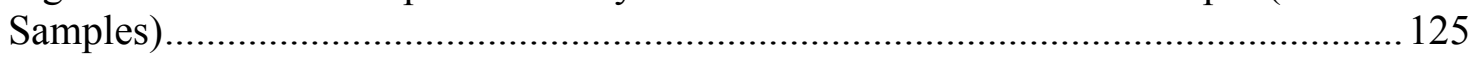

Figure 7-1 Schematic Representation of the Stress and Strain Components (After

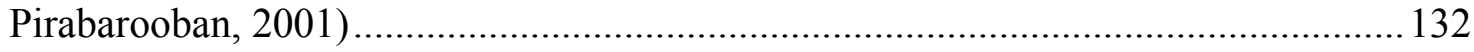

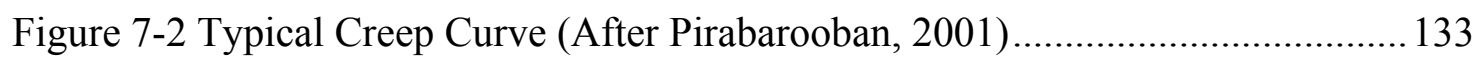

Figure 7-3 Simplified Loading Method (After Hua, 2000) ..................................... 138

Figure 7-4 NCAT Test Track Cross Section.................................................... 140

Figure 7-5 Four-node Bilinear Plane Strain Element (CPE4) ................................ 140

Figure 7-6 NCAT Test Track Boundary Conditions ............................................. 141

Figure 7-7 Effect of Elastic Foundation Stiffness............................................ 142

Figure 7-8 Track Pavement Stress Distribution.................................................. 143

Figure 7-9 Mesh Optimization Loading Configuration ......................................... 144

Figure 7-10 Effect of the Transverse Element Size (Test Track) ............................. 144

Figure 7-11 Effect of the Vertical Element Size (Test Track) ................................ 145

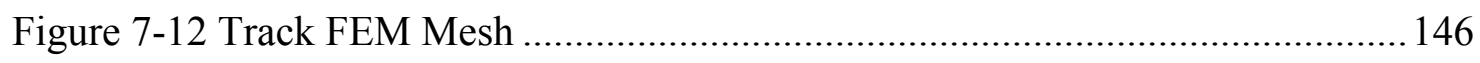

Figure 7-13 Total Rut Development at the NCAT Test Track (Neat Binders) ........... 147

Figure 7-14 Track Rut Depth Development .................................................. 148

Figure 7-15 Dual-wheel Assembly .......................................................... 151

Figure 7-16 Superposition of Dual-wheel Assembly (After Hua, 2000)................... 151

Figure 7-17 Loading Time Distribution for Wheel Wander (Test Track) .................. 152

Figure 7-18 Four-node Bilinear Plane Strain Element (CPE4) ............................... 153

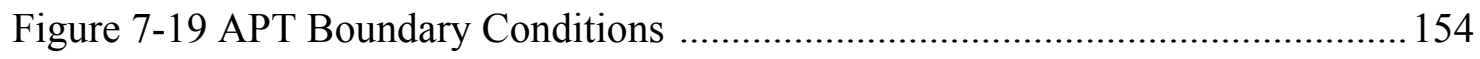

Figure 7-20 Effect of Elastic Foundation Stiffness............................................ 155 
$\begin{array}{ll}\text { Figure } & \text { Page }\end{array}$

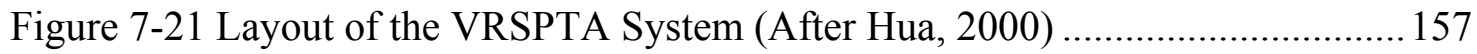

Figure 7-22 VRSPTA Diamond-shaped Effective area (After Hua, 2000) ................ 158

Figure 7-23 Contact Stress Distribution for Goodyear 11R22.5

$($ Tire Pressure $=620 \mathrm{kPa})($ Debeer and Fisher, 1997) 158

Figure 7-24 APT Tire Print................................................................................ 159

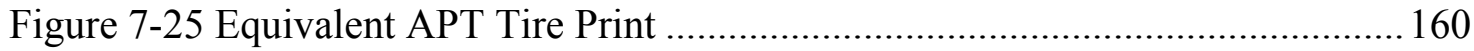

Figure 7-26 APT Contact Pressure Distribution................................................... 160

Figure 7-27 Effect of Transverse Direction Element Size (APT) ............................. 162

Figure 7-28 Effect of Vertical Direction Element Size (APT) .............................. 162

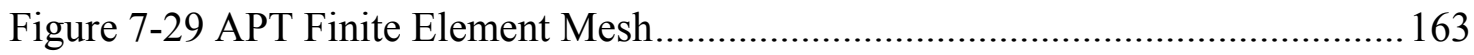

Figure 7-30 PURWheel Specimen Geometry (After Hua, 2000) ............................. 164

Figure 7-31 PURWheel Loading Configuration .................................................. 164

Figure 7-32 Eight-node Llinear Brick Element, C3D8R (After Hua, 2000) ............... 165

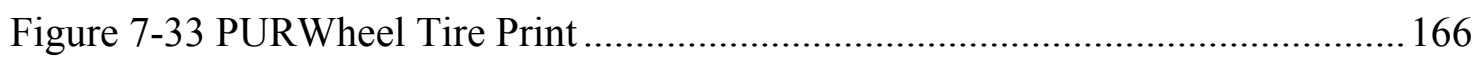

Figure 7-34 PURWheel Tire Print Conversion (After Hua,2000)........................... 167

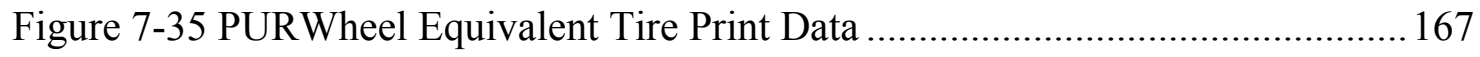

Figure 7-36 PURWheel Slab (quarter-size) used in the FEM ................................. 169

Figure 7-37 Effect of Transverse Direction Element Size (PURWheel)................... 170

Figure 7-38 Effect of Longitudinal Direction Element Size (PURWheel) ................. 170

Figure 7-39 Effect of Vertical Direction Element Size (PURWheel)........................ 171

Figure 7-40 Plan View of the PURWheel mesh .................................................. 171

Figure 7-41 Elevation View of the PURWheel mesh ......................................... 172 
$\begin{array}{lll}\text { Figure } & \text { Page }\end{array}$

Figure 7-42 Effect of Creep Parameter A on NCAT Test Track Rutting (FEM) ........173

Figure 7-43 Effect of Creep Parameter A on APT Rutting (FEM) .............................. 173

Figure 7-44 Effect of Creep Parameter A on PURWheel Rutting (FEM) ...................174

Figure 7-45 Effect of Creep Parameter m on NCAT Test Track Rutting (FEM) ........174

Figure 7-46 Effect of Creep Parameter m on APT Rutting (FEM) ……………….... 175

Figure 7-47 Effect of Creep Parameter m on PURWheel Rutting (FEM)................... 175

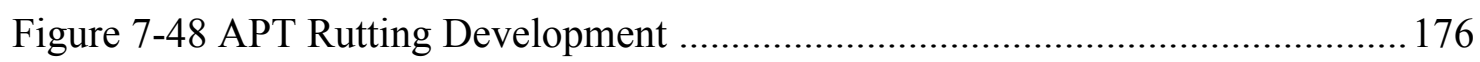

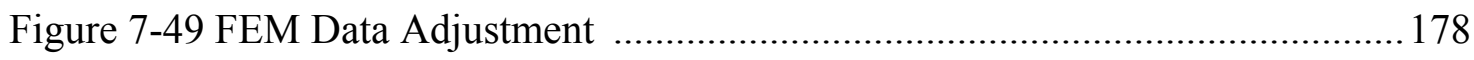

Figure 7-50 Rut Development in the N4 Test Track Section ..................................... 179

Figure 7-51 Parameter Determination for APT Section N3 .................................. 181

Figure 7-52 Parameter Determination for PURWheel Section N3 (Track Slabs) ....... 182

Figure 7-53 Comparison of Measured and Predicted Track Rut Depths

(APT Material Properties)........................................................................................ 183

Figure 7-54 Comparison of Measured and Predicted Track Rut Depth (PURWheel

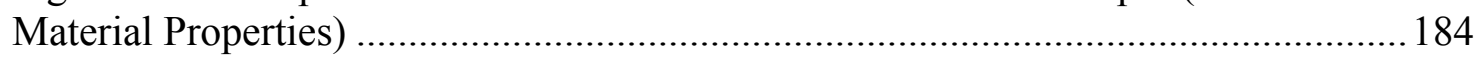

Figure 7-55 Comparison of Rutting Performance, Regions III and VI ...................... 186

Figure 7-56 Comparison of Rutting Performance, Regions III and VI .......................186

Figure 7-57 Effect of Temperature on Parameter m (APT) ………………………..... 187

Figure 7-58 Effect of Temperature on Parameter A (APT) ...................................... 188

Figure 7-59 Effect of Initial Density on Parameter $m$ at Different

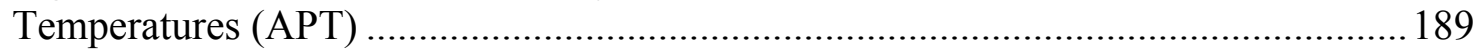

Figure 7-60 Effect of Initial Density on Parameter A at Different

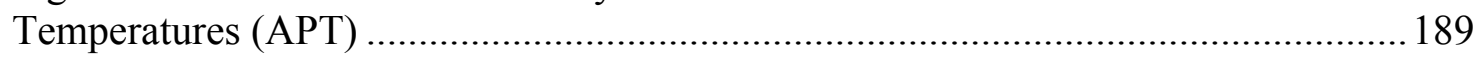

Figure 7-61 Variation of Parameter A with Temperature (Track, $1^{\text {st }}$ Summer) .......... 190 
Figure $\quad$ Page

Figure 7-62 Variation of Parameter m with Temperatures (Track, $1^{\text {st }}$ Summer)......... 191

Figure A-1 Rut Development in the N1 Test Track Section ...................................210

Figure A-2 Rut Development in the N2 Test Track Section ..................................210

Figure A-3 Rut Development in the N3 Test Track Section ..................................211

Figure A-4 Rut Development in the N5 Test Track Section ..................................211

Figure A-5 Rut Development in the N6 Test Track Section .................................212

Figure A-6 Parameter Determination for APT Section N1 .................................212

Figure A-7 Parameter Determination for APT Section N2 …............................. 213

Figure A-8 Parameter Determination for APT Section N4 ….............................213

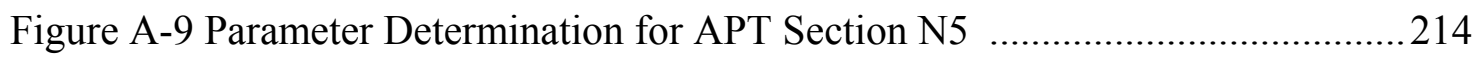

Figure A-10 Parameter Determination for APT Section N6 ................................ 214

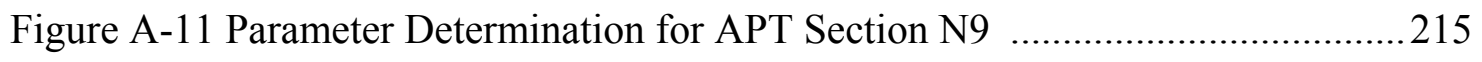

Figure A-12 Parameter Determination for APT Section N10 ............................... 215

Figure A-13 Parameter Determination for APT Section N1 (35C) ......................... 216

Figure A-14 Parameter Determination for APT Section N3 (35C) ......................... 216

Figure A-15 Parameter Determination for APT Section N4 (35C) ........................... 217

Figure A-16 Parameter Determination for PURWheel Section N1 (Track Slabs) ...... 217

Figure A-17 Parameter Determination for PURWheel Section N2 (Track Slabs) ...... 218

Figure A-18 Parameter Determination for PURWheel Section N4 (Track Slabs) ......218

Figure A-19 Parameter Determination for PURWheel Section N5 (Track Slabs) .....219

Figure A-20 Parameter Determination for PURWheel Section N6 (Track Slabs) ...... 219

Figure A-21 Parameter Determination for PURWheel Section N7 (Track Slabs) ...... 220 
Figure A-22 Parameter Determination for PURWheel Section N8 (Track Slabs) ..... 221

Figure A-23 Parameter Determination for PURWheel Section N1 (APT Slabs) ........222

Figure A-24 Parameter Determination for PURWheel Section N2 (APT Slabs) ........222

Figure A-25 Parameter Determination for PURWheel Section N4 (APT Slabs) ........223

Figure A-26 Parameter Determination for PURWheel Section N5 (APT Slabs) .......223

Figure A-27 Parameter Determination for PURWheel Section N6 (APT Slabs) ........224

Figure A-28 Parameter Determination for PURWheel Section N9 (APT Slabs) .......224

Figure A-29 Parameter Determination for PURWheel Section N10 (APT Slabs) ......225 


\section{CHAPTER 1 INTRODUCTION}

\subsection{Overview}

In 1987, the Strategic Highway Research Program (SHRP) was launched to improve the performance of hot-mix asphalt (HMA) pavements in the United States. One third of the money was allocated for research work focusing on asphalt materials. As part of the SHRP program, the Superior Performing Pavements (Superpave) mixture design and analysis system was developed. The Indiana Department of Transportation (INDOT) adopted this system and attendant specification in mid 1990’s. Though progress and benefits have been observed, long-term (20 years) performance data have not been available. When the National Center for Asphalt Technology (NCAT) began planning to build and operate a full-scale test track, INDOT saw an opportunity to further validate the Superpave design method and specifications. Additionally, the INDOT was interested in establishing rutting performance relationships between the Accelerated Pavement Tester (APT), the Purdue laboratory wheel-tracking device (PURWheel), and in-service pavements.

\section{$\underline{1.2 \text { Background }}$}

The highways in the United States are continuously subjected to increasing traffic volumes, loads, and tire pressure. In 1982, the legal load limit was increased from 326 to 
$356 \mathrm{kN}$ (73,280 to 80,000 lbs), resulting in approximately 40 to 50 percent higher pavement stresses for a given pavement structure (Superpave, 1996). The use of radial tires further increased the stress levels in the pavement structure. These changes resulted in an accelerated rate of pavement deterioration as pavement distresses such as rutting and fatigue developed more quickly. It became clear that older methods of pavement design and materials selection needed updating in order to extend the service life of HMA pavements (Metcalf, 1996).

As part of the attempt to update design methods, the Superpave mixture design and analysis system was developed. It includes performance models for three pavement distresses: rutting, fatigue and thermal cracking. However these models need calibration through actual traffic loading. Data collection from the existing pavements would take many years and other options were therefore considered. Additionally, many state agencies started using laboratory wheel tracking devices to predict pavement performance. Although, these devices predict rutting potential to a certain degree, further field validation was needed (Brown, 1998).

One option to collect performance data in a condensed amount of time is to construct and operate a test track loaded with actual trucks. This method should be most representative of actual highway loading. Therefore, the Alabama Department of Transportation (ALDOT) along with NCAT and the Federal Highway Administration (FHWA) decided to build and operate a test track near Opelika, Alabama. INDOT decided to participate in the study by sponsoring two sections. These two sections complement eight other test sections sponsored by the ALDOT and the FHWA. 


\subsection{Objectives}

The objectives of this research project are to validate the Superpave mixture design method and to establish rutting relationships between the APT, PURWheel, and in-service pavements (as represented by the test track).

\subsection{Scope}

The ten HMA mixtures included in this study were designed using the Superpave mixture design method and placed on the NCAT test track. Eight of the ten mixtures were duplicated and tested in the APT as well. In addition, PURWheel slabs were cut from the APT test sections as well as from the track and tested in the PURWheel. Loose mixture samples taken at the paver during APT construction were compacted in the Superpave Gyratory Compactor (SGC) and subjected to the dynamic modulus test. PURWheel specimens were also prepared in the laboratory using original aggregates and binders sampled during NCAT track construction; these specimens were tested to compare and contrast laboratory and field compaction.

HMA is a time, stress and temperature dependent material. Any efforts to characterize the material must account for these variables. A creep model was therefore selected and used to characterize the HMA mixtures. Finite element modeling (FEM) was used to determine material parameters for the creep model from the APT and PURWheel data and used as input for the NCAT FEM track model. By comparing FEM predicted and track-measured data, stronger rutting performance relationships between the APT, the PURWheel, and the NCAT test track were established. The factors that have 
significant effects on the rutting performance were identified and a better understanding of rutting phenomena was obtained. 


\section{CHAPTER 2 LITERATURE REVIEW}

\subsection{HMA Pavement Distresses}

HMA pavements primarily exhibit three types of distresses: permanent

deformation; fatigue; and thermal cracking.

\subsubsection{Permanent Deformation}

Permanent deformation is the accumulation of the irrecoverable strains resulting from the repeated load applications on HMA pavements and is characterized by depressions in the wheel paths and often with an uplift of the mixture between and at the sides of the wheel paths.

\subsubsection{Rutting Development Mechanism}

Generally two principal causes of rutting are identified; weak support layers, and weak or poor HMA mixtures (McGennis et al., 1995). The first case is a structural problem and occurs when pavement layers such as base, subbase and or subgrade are weakened by the high stresses acting on them as a result of traffic. It is often caused by an insufficient depth of HMA layer above the underlying layers or by moisture damage to the subgrade. In either case, the rutting occurs in one or more of the underlying layers. Figure 2-1 shows a diagram of rutting occurring in the subgrade. 


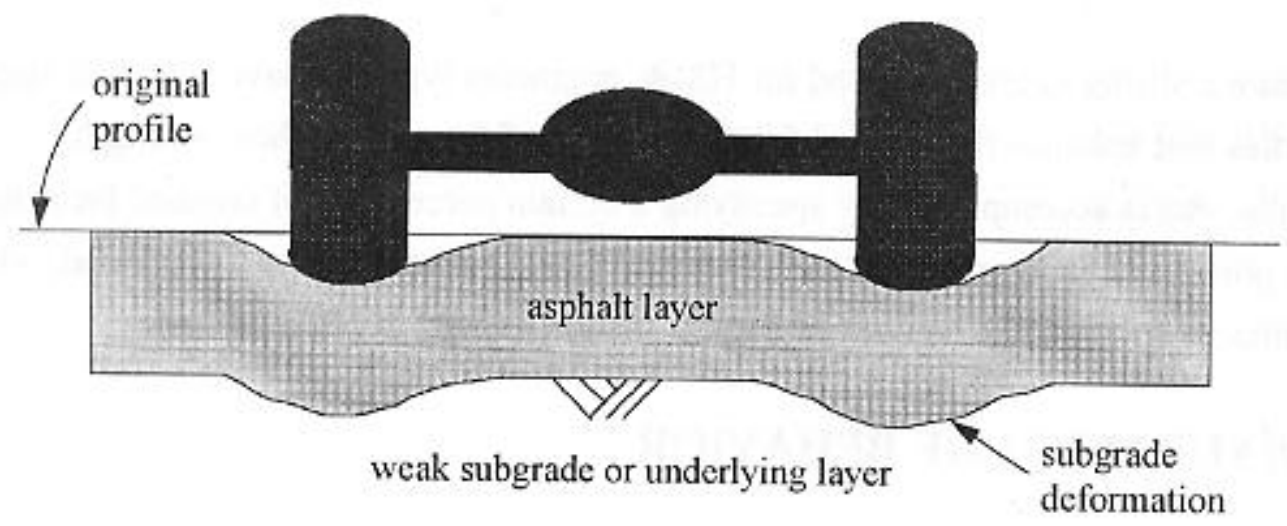

Figure 2-1 Rutting Caused by Weak Subgrade (After McGennis et al., 1995)

The second type of rutting occurs in the HMA mixture and can be a material and/ or construction problem. A schematic of this type of rutting is shown in Figure 2-2. HMA pavement rutting resulting from a poor performing HMA mixture is a temperature related phenomenon. Generally, more rutting is observed during the summer when pavement temperatures are elevated. The rutting in an HMA layer can occur due to consolidation (densification) and/or shear deformation (plastic flow).

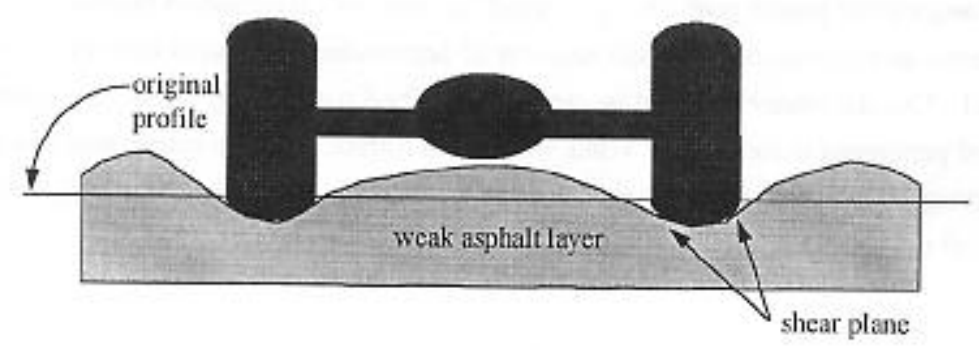

Figure 2-2 Rutting Caused by HMA Shear Failure (After McGennis et al., 1995)

Consolidation occurs in the HMA layer with a reduction in air voids due to traffic (Roberts et al., 1996). Typically, HMA mixtures are designed to have approximately 
three to five percent air voids at the design traffic level. However, HMA pavements are typically constructed to an initial air voids content of approximately 7 to 8 percent. Further, in some cases due to poor compaction, in-service pavements can have initial air voids ranging from 10 to 12 percent. With the application of traffic, further consolidation of the HMA mixture occurs gradually and the air voids in the mixture are reduced.

Downward and lateral movement of the HMA layer characterizes shear deformation (plastic flow). With the application of sufficient loads the shear deformation accumulates and rutting appears. Application of excessive asphalt binder can reduce the internal friction of the aggregates and can result in the binder attempting to carry applied loads rather than the aggregate skeleton (Roberts et al., 1996) (Mates, 1989). Trench studies of the test tracks and road studies reported that shear deformation is the primary factor causing rutting on properly constructed pavement (Hofstra and Klomp, 1972) (Highway Research Board, 1962).

Excessive rutting can cause a reduction in pavement service life and create unsafe conditions. Rutting in excess of $0.6 \mathrm{~cm}(0.25 \mathrm{in})$ on a pavement surface is typically considered a possible hydroplaning safety hazard by many state departments of transportation (Jackson and Baldwin, 1999). Recently, higher traffic volumes, traffic loads and the use of radial tires with higher inflation pressures have further aggravated the rutting problem. HMA mixtures that are prone to rutting therefore need to be identified and eliminated from use thus resulting in a savings to the highway industry. 


\subsubsection{Definitions and Measurement of Rutting}

To correctly quantify rutting, its components must be clearly understood. Total rut-depth is defined as the summation of rut depth (compactive rutting) and rise height (uplift) as shown in Figure 2-3. The rut depth is the vertical distance measured between the original surface and the lowest point of the surface profile. Rise height is defined as the vertical distance between the original surface and a point on the straight edge located directly above the depressed surface. The straight edge is constructed by connecting adjacent crest points on the profile.

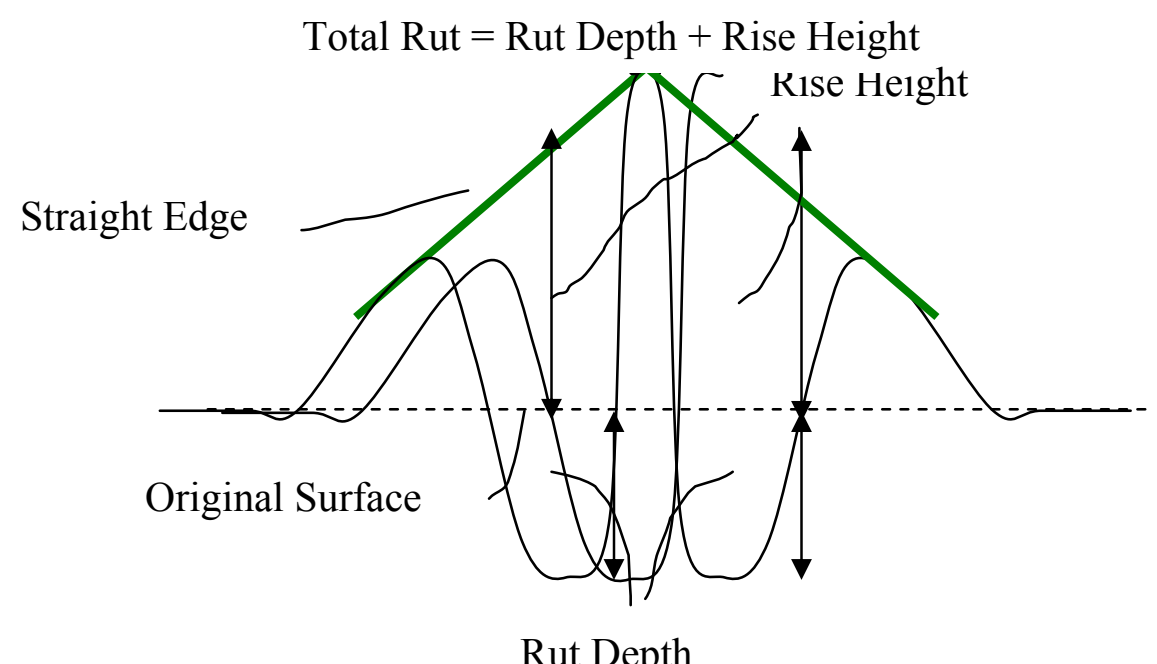

Figure 2-3 Rut Definition, No Wander (After Steiady, 2000)

Rutting is usually measured using profilometers. These devices record the profile data using horizontal and vertical transducers. An initial reference profile is generated before the application of the traffic and profile measurements are taken after various amounts of traffic have been applied. The rut depths caused by a given amount of traffic can thus be determined. Accurate rut depth measurements are important in order to make 
valid conclusions from the data. Currently, many methods are being used to measure pavement rutting. Among the most common are the rut-bar, dipstick, straightedge, rod and level, and trenching (Chen et al., 2001).

\subsubsection{Fatigue Cracking}

Fatigue cracking is a series of interconnected cracks formed as a result of the application of excessive wheel load repetitions or heavy loads on the HMA layer and or base (Roberts et al., 1996) (Huang, 1993). Fatigue traditionally develops at the bottom of the asphalt layer where tensile stresses and strains are higher and propagate up through the pavement. As additional cracks occur they tend to coalesce forming a pattern reminiscent of alligator hide, thus the term, "alligator cracking." When a pavement reaches this point it begins to experience failure.

Fatigue cracking resistance is dependent on binder content, binder viscosity, air voids, and the amount of aggregate fines (Epps and Quintus, 1990). Increasing the binder content initially results in improved fatigue resistance. However, once the binder content goes beyond a certain value, fatigue resistance decreases. Increasing air voids increases the fatigue resistance by reducing the mixture stiffness. Increasing binder viscosity and aggregate fines reduces the fatigue resistance by increasing mixture stiffness.

\subsubsection{Thermal Cracking}

Thermal cracking is the result of the shrinkage stresses that are induced in an HMA pavement by temperature changes. When these stresses exceed the tensile strength of the HMA mixtures in the pavement, the pavement is forced to crack to relieve the 
stress. Thermal cracking usually initiates at the pavement surface and propagates to underlying layers. It is mostly related to the binder characteristics at the lower temperatures (Badaruddin and White, 1995) (Galal and White, 2000). There are two variants of thermal cracking. The first is shrinkage cracking that occurs when the pavement temperature drops below a critical temperature. The second is thermal fatigue cracking that occurs due to repeated temperature cycling. Softer binders provide improved thermal cracking resistance while temperature susceptible binders have less resistance to thermal cracking.

\subsection{HMA Mixture Rutting Performance}

The factors affecting HMA rutting performance can be divided into three broad categories: 1) Aggregate properties; 2) binder properties; and 3) mixture properties.

\subsubsection{Aggregate Properties}

Gradation, shape, surface texture, and size are four aggregate properties that have been identified as properties that affect rutting performance.

\subsubsection{Aggregate Gradation}

Brown and Sniath (1974) and Monismith et al. (1985) reported that continuously, or dense-graded, mixtures have higher rutting resistance than gap-, or open-graded mixtures. Dense-graded aggregate blends produce HMA mixtures with fewer air voids and more aggregate contact points. These can increase the internal friction between 
aggregate particles thus increasing the HMA mixture stability and improving rut resistance.

There continues to be a debate whether coarse- or fine-graded HMA mixtures provide better rutting performance. Since the advent of the Superpave mixture design method, coarse-graded mixtures are those with a gradation passing below the restricted zone while fine-graded mixtures pass over it. The Superpave mixture design procedure originally recommended using coarse gradations. However, numerous state agencies had successfully used fine gradations as well as gradations passing through the restricted zone with adequate rutting performance prior to Superpave. The WesTrack experiment (Hand et al., 2000) also reported that fine-graded mixtures provide better rutting resistance than do coarse-graded mixtures as shown in Figure 2-4.

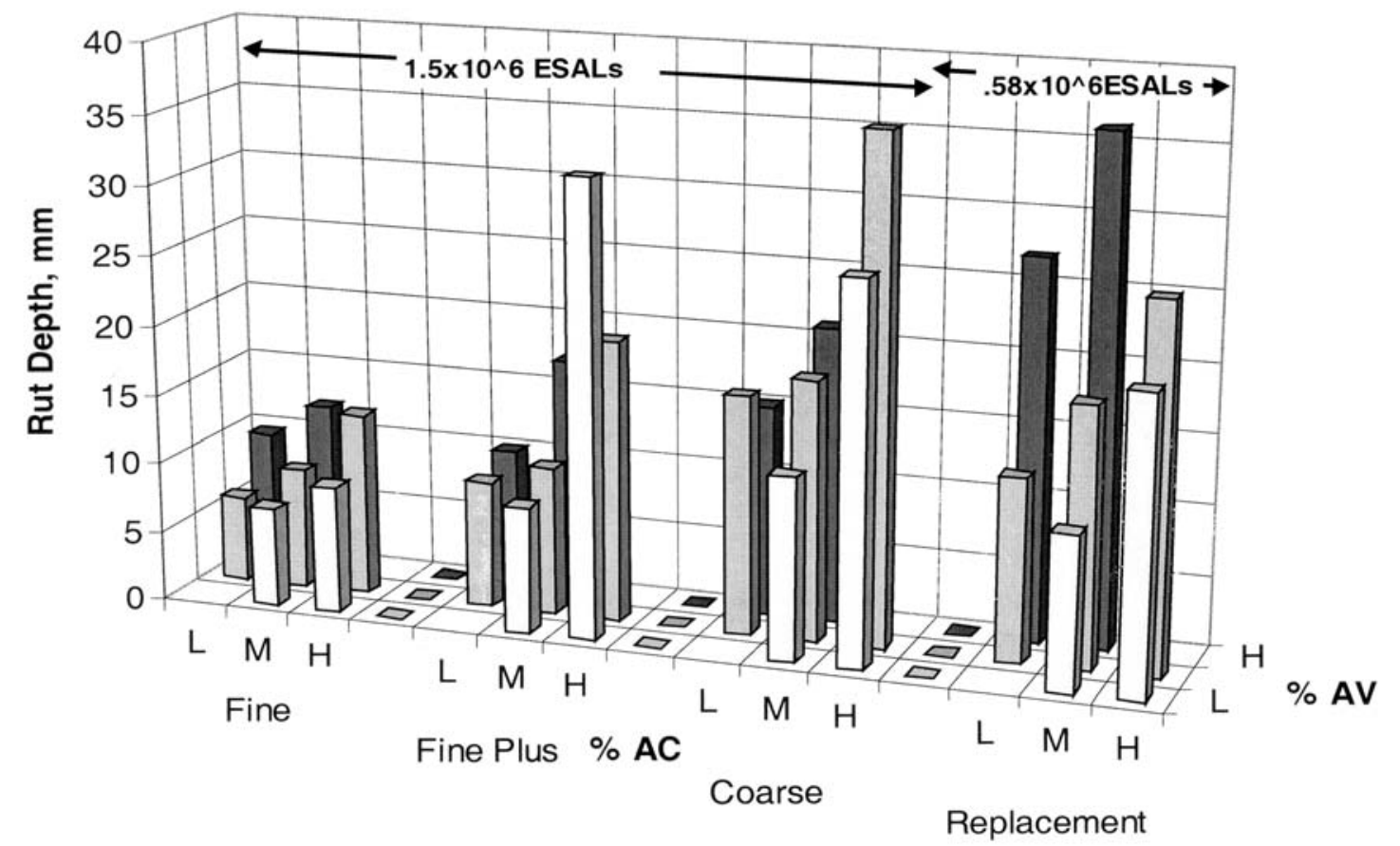

Figure 2-4 Effect of Gradation on Rutting at WesTrack (After Hand et al., 2000) 
The National Pooled Funds study No.176 was proposed to address the rutting performance of coarse and fine gradations (Stiady, 2000) (Stiady et al., 2003) (Hand et al., 2000). The results indicate that fine-graded mixtures provide slightly better rutting performance than do coarse-graded mixtures in the PURWheel test. However, no clear trends were observed in the APT tests. It was concluded that adequate rutting performance can be achieved using fine- or coarse-gradations, as well as gradations passing through the restricted zone. The researchers therefore recommended that the restricted zone should be omitted from the Superpave mixture design method.

\subsubsection{Aggregate Shape and Surface Texture}

Uge and Van de Loo (1974) reported that for a given gradation, HMA mixtures containing angular coarse aggregates exhibited improved rutting resistance over mixtures containing rounded coarse aggregates (river gravel). Aggregates that are angular and rough textured have higher internal friction and consequently provide better rut resistance than aggregates that are rounded and smooth. Therefore, mixtures made from crushed aggregates tend to provide higher rut resistance than do mixtures containing uncrushed materials.

The inclusion of excessive natural sand can also result in HMA mixtures that are prone to rutting as reported by many researchers (Brosseaud et al., 1993) (Galal and White, 1999). Brosseaud et al. (1993) reported as much as a 50 percent increase in rutting in HMA mixtures when crushed sand was replaced by uncrushed natural sand. Natural sand is usually rounded and produces a weak aggregate skeleton. 


\subsubsection{Dust Proportion}

The dust is considered to be the portion of aggregate material that passes the 0.075-mm (No. 200) sieve. The amount of dust in an HMA mixture can affect a mixture's rutting performance and therefore the Superpave mixture design and specification system uses a parameter called the dust proportion (dust to asphalt binder ratio) to limit the amount of aggregate dust in an HMA mixture (Superpave, 1996). If too much aggregate dust is used (higher dust proportion), durability may be a problem. If too low aggregate dust is used (lower dust proportion), stability may be a concern. The initial recommendation of dust proportion was a ratio of 0.6 to 1.2 , but was later changed to be 0.6 to 1.6 .

Christensen and Bonaquist (2005) reported that aggregate fines will improve rutting resistance of an HMA mixture and aggregate dust smaller than 2 microns can act as an extender to binder. If too much of aggregate dust smaller than 2 microns were used, stability may be a concern.

\subsubsection{Nominal Maximum Aggregate Size}

The nominal maximum aggregate size (NMAS) is defined by the Superpave system to be one sieve size larger than the first sieve to retain more than ten percent of the aggregate. Increasing the NMAS can often improve the rutting performance of HMA mixtures. For example, Davis (1988) reported that HMA pavements made from HMA mixtures that used soft binders, high volumes of aggregate, low air voids, and large NMAS aggregates showed better rutting resistance over those using smaller NMAS aggregates. Based on the results, he concluded that using larger aggregates would provide 
benefits in terms of rutting performance, especially on heavy-duty pavements. Similarly, Bonaquist et al. (1998) reported that increased NMAS in HMA mixtures resulted in improved rutting performance in full-scale accelerated rutting tests.

\subsubsection{Binder Properties}

For a given HMA mixture, increasing the viscosity of the asphalt binder in the mixture increases the mixture stiffness. The stiffer mixture therefore provides better rutting resistance (Monismith et al., 1985) (Brosseaud et al., 1993). While unmodified binders can often provide adequate stiffness, modified binders typically provide higher stiffness at high temperatures and can result in improved rutting performance. Modified binders are obtained when polymers or other such additives are incorporated into an unmodified binder. Monismith and Tayebeli (1988) reported improved rutting performance when an AR-2000 binder was modified using carbon black.

\subsubsection{Mixture Properties}

Mixture properties that can affect HMA mixture rutting performance include binder content, air void content, voids in the mineral aggregate (VMA), and voids filled with asphalt (VFA).

\subsubsection{Binder Content}

Too much binder in an HMA mixture can make the mixture prone to rutting. This is one factor causing plastic flow of HMA mixtures (Roberts et al., 1996). Excessive binder content causes a reduction in the aggregate particle interlock causing them to 
"float" in the binder rather than lock together. When this occurs, the binder is forced to carry the applied loads rather than the aggregate skeleton. Several research studies (Galal and White, 1999) (Archilla and Madanat, 2000) have shown that higher binder contents can result in poorer rutting resistance. Galal and White (1999) reported that increasing the binder content by $0.25 \%$ resulted in an approximately 40 percent increase in the rutting potential for gravel mixtures.

\subsubsection{Air Void Content}

Air void content, the volume of air present within a compacted HMA mixture, is one of the most important properties in determining the rutting performance of HMA mixtures. Too high an air void content can result in higher potential for stripping, oxidation, and densification rutting. Too low an air void content can result in higher potential for bleeding and shear flow rutting a mixture becomes unstable (Brown and Cross, 1992) (Harrigan, 2002). Typically, initial air void contents ranging from seven to nine percent are recommended for in-service pavements. The Superpave mixture design method requires that the optimum binder content be chosen to provide four percent air voids at the design compaction level.

\subsubsection{Voids in the Mineral Aggregate}

The VMA is the total volume of voids in a compacted aggregate and can considerably affect a mixture's rutting performance. VMA consists of two components, the volume of air voids and the volume of effective (unabsorbed) binder. The effective volume of binder is critical to the durability of HMA since the effective volume of binder 
and aggregate gradation determine the binder film thickness on the mineral aggregate (Roberts et al., 1996). Adequate binder film thickness is required to protect HMA mixtures against oxidation and the effects of moisture. For this reason, minimum VMA requirements have been established. Since smaller NMAS aggregates result in higher aggregate surface area, the minimum VMA requirements increase with decreasing NMAS of the aggregate. Stiady et al. (2003) reported that the relationship between VMA and the NMAS is greatly dependent on the fine aggregate and mixture gradation and that the minimum VMA requirements alone do not differentiate between stable and unstable HMA mixtures.

VMA that is too high would result in thicker binder film thickness, if excessive binder were used in the mixture. Consequently, aggregate particle interlocks can be reduced and traffic loads are resisted by binder instead of aggregate skeleton, resulting in lower rutting resistance. Therefore, for improved mixture performance, an optimum VMA is required.

\subsubsection{Voids Filled with Asphalt}

The VFA is the percent of VMA that is filled with binder. As such, air void content, VMA, and VFA are inter-related. If any two are controlled, the third is automatically controlled. As HMA mixtures are exposed to traffic loading a reduction in air voids can results thus decreasing the VMA and increasing the VFA. For warmer climates, higher binder content can be more prone to shear flow rutting since thermal expansion of the binder need more space to fill. Therefore, the VFA is limited to 65 to 80 percent depending on the design traffic level. 


\subsection{Accelerated Pavement Testing}

Accelerated pavement tests generally apply full-scale or partial scale traffic loads in an accelerated manner with or without environmental control. The result is that normal pavement distresses can occur in a condensed time frame. Three types of accelerated pavement testers are included in this study: 1) Full-scale test track; 2) full-scale accelerated loading devices; and 3) laboratory wheel tracking devices.

\subsubsection{Full-Scale Test Tracks}

Test tracks apply actual traffic loads to test sections under varying seasonal temperatures in an accelerated manner. Many test track studies have been completed or are still being conducted to enhance pavement performance. The American Association of State Highway Officials (AASHO) road test is perhaps the most well-known test road in the world and was performed in Ottawa, Illinois from 1958 through 1960 (Highway Research Board, 1962) (Huang, 1993) (Metcalf, 1996) (Brown and Powell, 2001). The objective of the test was to establish relationships among the repetitions of varying axle loads and configurations to pavement performance.

To meet the project objective, vehicles with varying loads and configurations were driven over pavement sections of assorted thicknesses as shown in Table 2-1. Six pavement tangents, each consisting of two lanes and varying in length from $1340 \mathrm{~m}$ (4400 ft) to $2070 \mathrm{~m}(6800 \mathrm{ft})$ were tested. North tangents were surfaced with HMA, while south tangents were surfaced with portland cement concrete (PCC). The tangents were further divided into test sections $30 \mathrm{~m}$ (100 ft) long. Over two years 1.114 million axle loads were applied and the performance data collected was used to develop the American 
Association of State Highway and Transportation Officials (AASHTO) pavement design guide.

Table 2-1 Loading of AASHO Road Test (After Huang, 1993)

\begin{tabular}{|l|c|c|c|c|c|c|c|c|c|c|c|c|}
\hline Loop No & \multicolumn{2}{|c|}{1} & \multicolumn{2}{|c|}{2} & \multicolumn{2}{|c|}{3} & \multicolumn{2}{|c|}{4} & \multicolumn{3}{|c|}{5} & \multicolumn{3}{|c|}{6} \\
\hline Lane No & 1 & 2 & 1 & 2 & 1 & 2 & 1 & 2 & 1 & 2 & 1 & 2 \\
\hline $\begin{array}{l}\text { Axle } \\
\text { Load(kN) }\end{array}$ & None & None & 0.45 & 1.35 & 2.7 & 5.4 & 4.0 & 7.2 & 5.0 & 9.0 & 6.7 & 10.8 \\
\hline $\begin{array}{l}\text { Axle } \\
\text { Type }\end{array}$ & None & None & $\mathrm{S}$ & $\mathrm{S}$ & $\mathrm{S}$ & $\mathrm{T}$ & $\mathrm{S}$ & $\mathrm{T}$ & $\mathrm{S}$ & $\mathrm{T}$ & $\mathrm{S}$ & $\mathrm{T}$ \\
\hline
\end{tabular}

$\mathrm{S}=$ Single, $\mathrm{T}=$ Tandem

Trench studies of the pavement sections conducted after the traffic loading concluded that HMA mixture rutting develops primarily due to the lateral movement of the materials rather than an increase in the density and that rutting in layers other than HMA accounts for only 9 percent of the total rutting. The study also concluded that increasing vehicle speed from 3 to $56 \mathrm{kph}$ ( 2 to $35 \mathrm{mph}$ ) resulted in a 38 percent reduction in pavement deflection.

The Pennsylvania Transportation Institute (PTI) research facility was built and operated between 1971 and 1983 (Metcalf, 1996). It included a test road $1.6 \mathrm{~km}$ (1 mile) long that served as a satellite station to the AASHO road test. Both flexible and rigid pavement sections were tested using different tractor-trailer combinations in four experimental cycles. During the first series of experiments, 17 test sections were tested. The loading was achieved using a 5-axle tractor-trailer combination loaded to $72-190 \mathrm{kN}$ $(16,000-24,000 \mathrm{lbs})$ and traveling at $36 \mathrm{kph}(22.3 \mathrm{mph})$. The structural numbers of the pavements were estimated from the data and resulted in lower than proposed structural coefficients. The study also compared three linear elastic layer models used to predict 
pavement performance. They were CHEVRON-1963, CRANLAY-1971, and BISAR1974. The Visco-elastic System model II (VESYS II) was also used. The study reported that the models predicted surface deformations within 10 percent.

A test track facility funded by the Minnesota Department of Transportation (MNDOT) and known as Mn/ROAD has recently been used to study the effects of materials, climate, and traffic on pavement performance in cooler climates (Metcalf, 1996) (Mulvaney and Worel, 2002). The study was also intended to serve as a basis for a mechanistic-empirical pavement design system for Minnesota.

The Mn/Road research project started in 1993 and consists of two pavements sections. One is a 4.8-km (3-mi), two-lane highway used for the heavy traffic application (mainline). The second is a 4-km (2.5-mi) closed loop, two-lane highway used for low traffic application. The 40 test sections, each $143 \mathrm{~m}$ (492 ft) long, were initially surfaced with asphalt, concrete, and gravel. Heavy traffic loading is achieved by diverting westbound interstate I-94 traffic while test trucks are used to traffic the low volume loop. Total wheel loads of the test trucks of the low volume loop vary between 356 and $454 \mathrm{kN}$ $(80,000$ to $102,000 \mathrm{lbs})$ and are applied at velocities ranging from 56 to $104 \mathrm{kph}$ (35 to 65 $\mathrm{mph})$.

A significant aspect of the $\mathrm{Mn} / \mathrm{Road}$ study is the use of extensive instrumentations including pressure cells, strain gauges, moisture blocks, time-domain reflectrometry, neutron probe, and thermocouples. The stain gauges have provided a means for comparison of measured strains and estimated strains at the bottom of the HMA layers using WESLEA (Waterways Experiment Station Layered Elastic Analysis) (Chadbourn 
et al., 1997). The results have confirmed the reasonableness of the WESLEA estimates and WESLEA is used in the MNDOT pavement design software.

Extensive laboratory tests were performed on the materials to provide a database for the material properties that can be used in a flexible pavement design system (Gardener, 1997). The study also reported that HMA mixture containing PG 64-22 binder rutted 59 percent less than did mixture containing PG 58-28 binders when placed in the driving lane. Two Superpave mixtures were placed on the Mn/Road in 1997 (Zerfas, 2003). A 100-mm (4-in.) overlay was placed above the existing $225 \mathrm{~mm}$ (9 in.) full-depth HMA on clay. One mixture is coarsely graded while the other has a gradation going through the restricted zone. A single binder grade (PG 58-22) was used for both mixtures. After 3 million Equivalent Single Axle Load (ESAL) applications, the observed rutting in both sections was similar, approximately $4.5 \mathrm{~mm}(0.2 \mathrm{in}$.$) .$

The WesTrack was constructed and tested in the mid 1990's near Reno, Nevada (Hand, 1998) (Epps et al., 1998, 1999). The primary purpose of this study was to “establish performance-based specifications and validation of the Superpave mix design analysis system.” WesTrack was a 2.8-km (1.8-mile) oval track consisting of 26 original test sections, each $70 \mathrm{~m}$ (230 ft) long. The layout is shown in Figure 2-5. Three levels of binder content and in-place density, and three aggregate gradations were included in the experimental design of the study as shown in Table 2-2. A crushed gravel aggregate source was used to produce a fine-gradation, a fine-plus gradation, and a coarsegradation. The HMA mixtures were designed using Superpave volumetric design method. Binder content, in-place density, and aggregate gradations were varied in an organized 
manner to simulate variables associated with construction. The target gradations are shown in Figure 2-6.

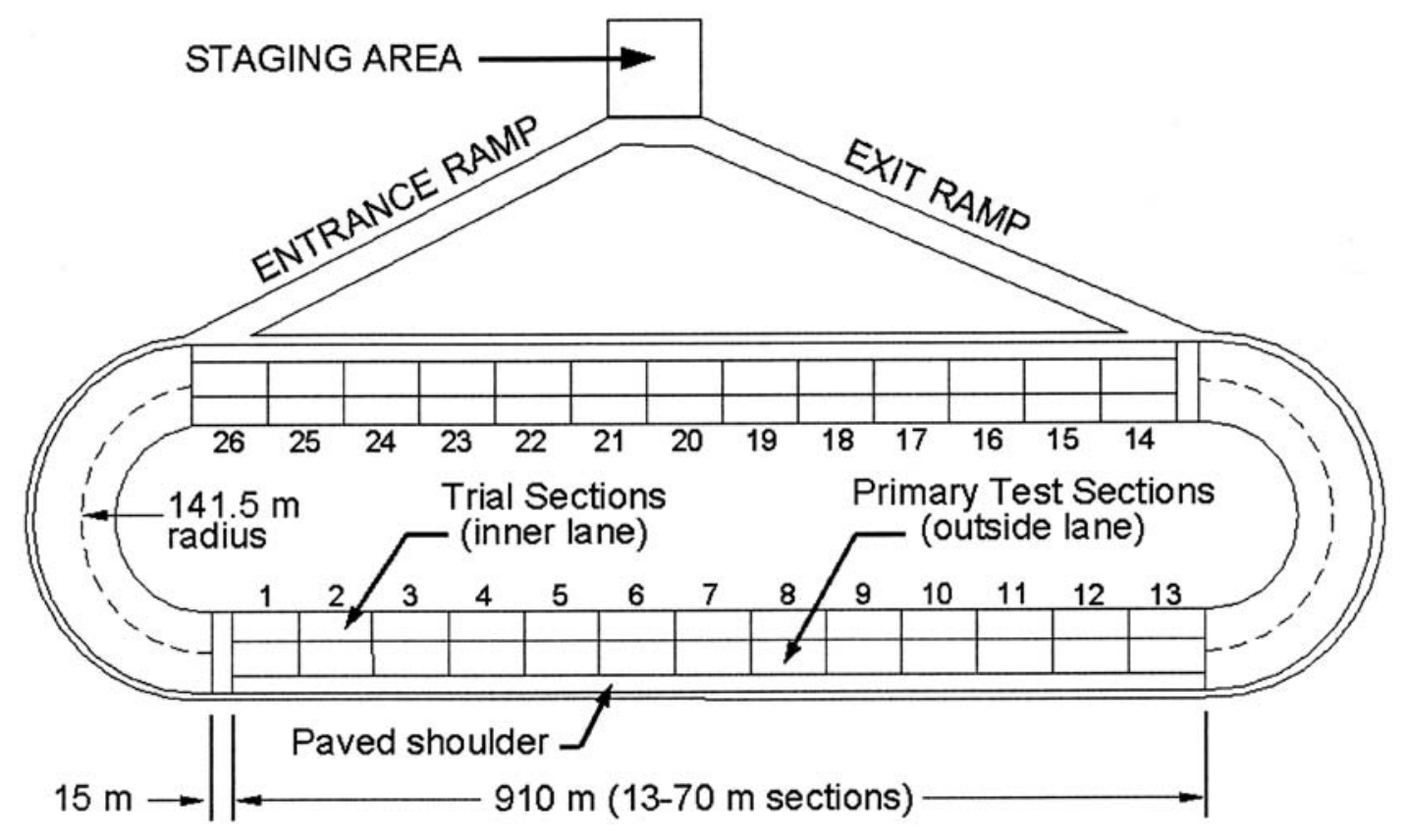

Figure 2-5 WesTrack Layout (After Epps et al., 1998)

Table 2-2 Experimental Design of the Original WestTrack Sections (After Epps et al., 1998)

\begin{tabular}{|c|c|c|c|c|c|c|c|c|c|}
\hline \multirow{4}{*}{$\begin{array}{c}\text { Air } \\
\text { Void } \\
\text { Content } \\
(\%)\end{array}$} & \multicolumn{9}{|c|}{ Aggregate Gradation } \\
\hline & \multicolumn{3}{|c|}{ Fine } & \multicolumn{3}{|c|}{ Fine plus } & \multicolumn{3}{|c|}{ Coarse } \\
\hline & \multicolumn{9}{|c|}{ Binder Content (\%) } \\
\hline & Low & Opt. & High & Low & Opt. & High & Low & Opt. & High \\
\hline Low & $\mathrm{X}$ & 04 & 18 & $\mathrm{X}$ & 12 & $09 / 12$ & $\mathrm{X}$ & 23 & 25 \\
\hline Medium & 02 & $01 / 15$ & 14 & 22 & $11 / 19$ & 13 & 08 & $05 / 24$ & 07 \\
\hline High & $03 / 16$ & 17 & $\mathrm{X}$ & 10 & 20 & $\mathrm{X}$ & 26 & 06 & $\mathrm{X}$ \\
\hline
\end{tabular}

Numbers in each cell indicate actual test section numbers.

$\mathrm{X}$ indicates no sections available for that cell. 


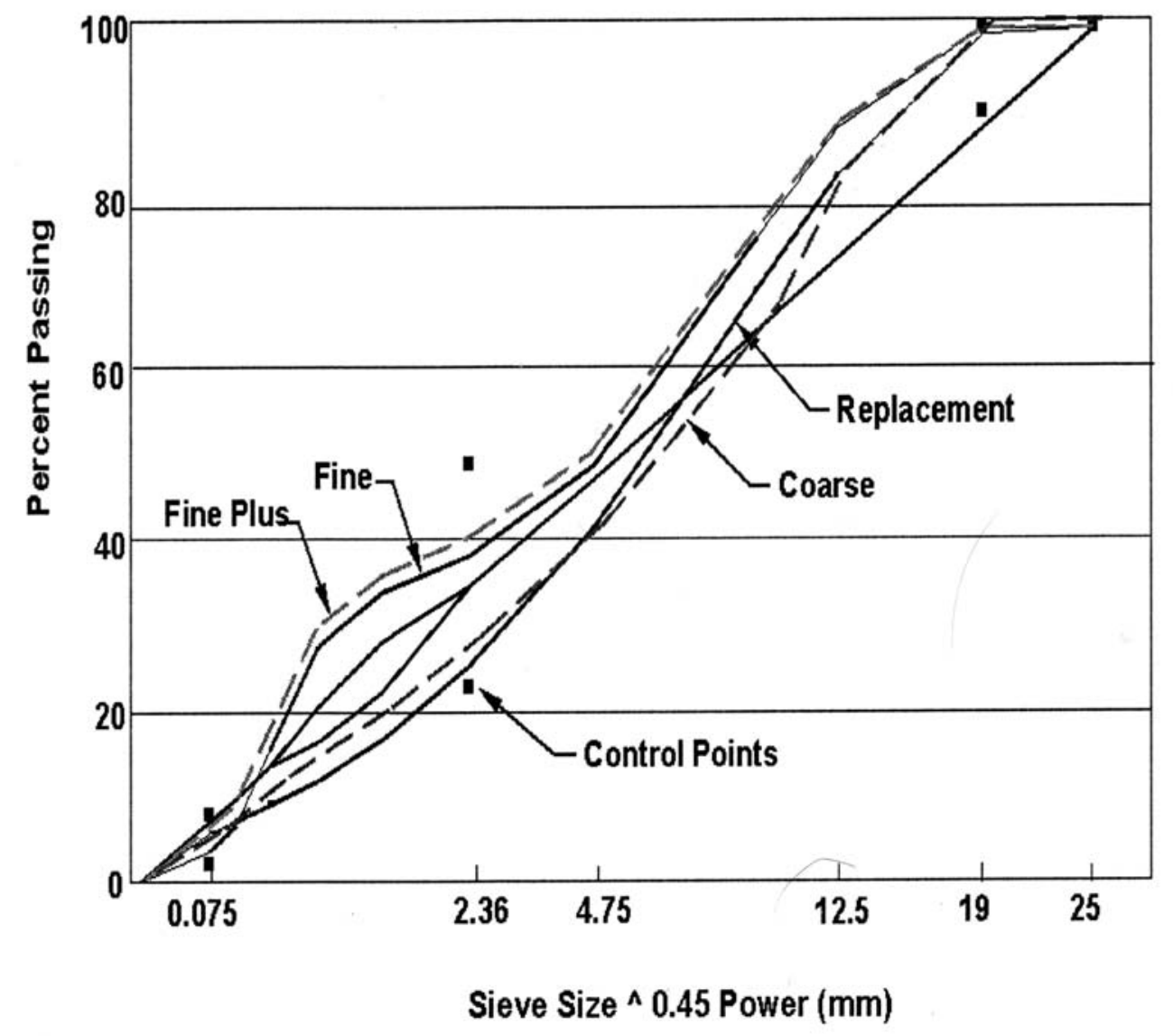

Figure 2-6 Target Gradations of WesTrack HMA Mixtures (After Epps et al., 1998)

The pavement structure consisted of a $150-\mathrm{mm}$ (6 in.) surface layer, a 150-mm (6 in.) dense graded aggregate base, a 300-mm (12 in.) engineered fill and a uniform siltyclay subgrade. The pavement structure was designed so that rutting and fatigue failure would occur within the time frame of the project. Traffic was applied using four tractortrailers combination, operated by computer-controlled methods. Each pass of the truck assembly applied approximately $10.5 \mathrm{ESAL}$ at a speed of $64 \mathrm{kph}(40 \mathrm{mph})$ with wheel wander. The truck tires were inflated to $700 \mathrm{kPa}$ (100 psi). Rutting, and fatigue 
performance data was collected every two weeks. Rutting performances of the WesTrack mixtures are shown in Table 2-3.

Table 2-3 WesTrack Rut Depth Summary (After Epps et al., 1998)

\begin{tabular}{|c|c|c|c|c|c|}
\hline \multirow{2}{*}{$\begin{array}{l}\text { Section } \\
\text { Number }\end{array}$} & \multicolumn{2}{|c|}{ Designation } & \multirow{2}{*}{$\begin{array}{l}\text { Rut Depth, } \\
\mathrm{mm}\end{array}$} & \multirow{2}{*}{$\begin{array}{l}\text { ESALs, } \\
\times 10^{6} * *\end{array}$} & \multirow{2}{*}{$\begin{array}{c}\text { Status } \\
\text { (after } 2.8 \text { million ESALs) }\end{array}$} \\
\hline & Gradation & AC/AV Content * & & & \\
\hline 01 & Fine & MM1 & 9 & 2.8 & In Service \\
\hline 02 & Fine & LM & 6 & 2.8 & In Service \\
\hline 03 & Fine & LH1 & 10 & 2.8 & In Service \\
\hline 04 & Fine & $\mathrm{ML}$ & 9 & 2.8 & In Service \\
\hline 05 & Coarse & MM1 & 22 & 2.8 & Out of Test \\
\hline 06 & Coarse & $\mathrm{MH}$ & 30 & 2.8 & Out of Test \\
\hline 07 & Coarse & $\mathrm{HM}$ & 36 & 1.5 & Out of Test \\
\hline 08 & Coarse & LM & 23 & 2.8 & Out of Test \\
\hline 09 & Fine Plus & HL2 & 30 & 1.5 & Out of Test \\
\hline 10 & Fine Plus & $\mathrm{LH}$ & 12 & 2.8 & In Service \\
\hline 11 & Fine Plus & MM2 & 11 & 2.8 & In Service \\
\hline 12 & Fine Plus & ML & 10 & 2.8 & In Service \\
\hline 13 & Fine Plus & $\mathrm{HM}$ & 20 & 1.5 & Out of Test \\
\hline 14 & Fine & $\mathrm{HM}$ & 10 & 2.8 & In Service \\
\hline 15 & Fine & MM2 & 10 & 2.8 & In Service \\
\hline 16 & Fine & LH2 & 9 & 2.8 & In Service \\
\hline 17 & Fine & MH & 10 & 2.8 & In Service \\
\hline 18 & Fine & $\mathrm{HL}$ & 7 & 2.8 & In Service \\
\hline 19 & Fine Plus & MM1 & 10 & 2.8 & In Service \\
\hline 20 & Fine Plus & $\mathrm{MH}$ & 11 & 2.8 & In Service \\
\hline 21 & Fine Plus & HL1 & 35 & 1.5 & Out of Test \\
\hline 22 & Fine Plus & LM & 10 & 2.8 & In Service \\
\hline 23 & Coarse & ML & 12 & 2.8 & In Service \\
\hline 24 & Coarse & MM2 & 26 & 2.8 & Out of Test \\
\hline 25 & Coarse & $\mathrm{HL}$ & 27 & 1.5 & Out of Test \\
\hline 26 & Coarse & LH & 19 & 2.8 & Out of Test \\
\hline
\end{tabular}

** For out-of-test sections, this is the cumulative ESALs at the time the section was removed from test.

Eight coarse-graded mixtures were replaced due to premature failure. These sections exhibited severe rutting of more than $25 \mathrm{~mm}$ (1 in.) after the application of only 2.8 million ESAL. It was hypothesized that the use of partially crushed gravels lead to the poor rutting performance of the mixtures and therefore more angular aggregates were used for the replacement sections. The newly placed replacement sections also exhibited 
significant rutting within first five days of trafficking (Brown et al., 1998). This concern resulted in a forensic investigation by a team of pavement researchers to determine the likely cause of premature rutting failure of the coarse-graded mixtures. A test program was completed to determine the in-place mixture properties (gradations, densities, binder contents, and mixture volumetrics) of the replacement sections and to compare them to the original production data. The mixture volumetric data indicated that the replacement mixtures did meet the Superpave requirements. However, these mixtures had high VMA and binder contents that could have increased rutting potential. The better performing mixtures had higher dust to binder ratios. The forensic investigation led to several recommendations for modifications to the Superpave mixture design system and specifications. Chief among these were the use of a maximum VMA in addition to the minimum value, use of a laboratory performance test to supplement volumetric design, and an increase in the upper range of the dust to bonder ratio for coarse-graded mixtures.

Based on the rutting performance of the HMA mixtures at the WesTrack other conclusions were also drawn. Higher air-voids resulted in slightly lower rutting resistance and mixtures with higher binder content experienced lower rutting resistance. Further, the rutting rate increases above a certain critical high temperature regardless of the mixture type. It was therefore concluded that the use of a critical high temperature for laboratory tests is important so that rutting performance might be predicted. The fatigue data showed that coarse-graded mixtures exhibited lower fatigue resistance compared to fine and fineplus graded HMA mixtures. Also, HMA mixtures with lower binder content showed less fatigue resistance than those with higher binder contents. HMA mixtures with higher air void contents exhibited lower fatigue resistance. As part of the WesTrack study, a series 
of regression rutting models were developed. These are discussed in section 2.4.3 of this report.

Although some Superpave validation efforts were completed through the WesTrack study, other issues need additional research work. For instance, the experimental design of the study was limited to only one binder type and one aggregate source. The calibration of the performance models should be validated over a range of aggregate sources and binder types. Further, it was observed that contrary to the study's expectation, the fine-graded mixtures performed better than the coarse-graded mixtures in the highly trafficked areas (Brown et al., 1998).

The limitations of the Westrack study, the need for the evaluation of the loaded wheel testing (LWT) devices for performance prediction, as well as the calibration needs of the SHRP performance models resulted in an increased interest in the design and operation of yet another test track (Brown, 1998). Such a test track could also identify the best materials and construction methods that could result in longer lasting HMA pavements. After investigating available options, NCAT along with ALDOT decided to build and test an oval-shaped test track near Opelika, Alabama. Several other states, including INDOT, were also interested in participating in the construction and funding the operation of the track.

\subsubsection{Full-Scale Accelerated Loading Devices}

These types of devices typically apply full-scale traffic loads using some type of machine rather than actual truck traffic to accelerate the damage to test lanes. Occasionally the environment of the system is also controlled. This class of devices 
includes the facility at the Laboratoire Central des Ponts et Chausse'es (LCPC) in France, South African Heavy Vehicle Simulator (HVS), Caltrans Mobile Load Simulator, the Accelerated Pavement Tester in Indiana, Texas Mobile Load Simulator (TxMLS) and various Accelerated Loading Facilities (ALF) including those in Australia, the FHWA ALF, Kansas State University, Louisiana, and Florida.

In the 1970's the French LCPC began operating a full-scale accelerated loading facility in Nantes, France. The facility applies loads ranging from 40 to $140 \mathrm{kN}(9,000$ to $31,500 \mathrm{lbs})$ on a circular test track made of pavement sections that are $120 \mathrm{~m}(400 \mathrm{ft})$ long and $6 \mathrm{~m}(20 \mathrm{ft})$ wide. Standard truck axles can be mounted on four arms that rotate at speeds between 30 and $100 \mathrm{kph}$ (19 to $63 \mathrm{mph}$ ). Among other research, the LCPC facility was used to investigate the effect of binder type on rutting performance (Brosseaud et al., 1993). Compared to the neat binders, modified binders exhibited improved rutting resistance in both laboratory wheel-tracking tests and in the full-scale LCPC facility.

An HVS is in use in South Africa since 1971 (Metcalf, 1996). Traditionally the South African HVS was used on in-service pavements and therefore evaluation of rehabilitation techniques is more common (Hugo and Epps, 2004). However, limited materials evaluation was also performed particularly involving base materials. Tests can be conducted on test sections $8 \mathrm{~m}(27 \mathrm{ft})$ long by $1.5 \mathrm{~m}(5 \mathrm{ft})$ also. Half axle single or dual tires can apply wheel loads varying from 20 to $100 \mathrm{kN}$ (4500 to $22500 \mathrm{lbs}$ ). The wheel velocity is $12 \mathrm{kph}(7.5 \mathrm{mph})$. Radiant heating is used to maintain temperature in the test area along with water. 
The California Department of Transportation (Caltrans) is using two HVS machines that have the same features as that of the South African HVS for its accelerated pavement testing since 1994. A study conducted with this equipment reported that a gapgraded, crumb-rubber-modified binder overlay exhibited improved rutting resistance performance over dense-graded mixture with a conventional binder when underlying layers have adequate rutting resistance (Harvey and Popescu, 2000).

The ALF was originally developed and is still in use in Melbourne, Australia. It applies a half axle load ranging from 40 to $80 \mathrm{kN}(9,000$ to $18,000 \mathrm{lbs})$ on a dual wheel assembly. The carriage speed varies from 1 to $20 \mathrm{kph}(0.5$ to $13 \mathrm{mph})$ and the usual operating speed is $20 \mathrm{kph}(13 \mathrm{mph})$. Using skirting and heat lamps, temperatures can be controlled between ambient and 60C (140F). The ALF has been used to investigate the effects of binder type and aggregate gradations (filler contents) on rutting performance (Sharp et al., 1999). The study reported that modified binders outperformed the neat binders with respect to the rutting resistance. Good correlation was reported between the full-scale testing and laboratory wheel tracking rutting performance. The Superpave binder rutting parameter $\mathrm{G}^{*} / \sin \delta$ showed a good correlation with rutting performance for the neat binders. However, its use as a rutting indicator was invalid for modified binders. Dynamic creep tests conducted on field cores at the ALF testing temperature did not rank the rutting performance correctly compared to the full-scale loading tests.

Many accelerated pavement-testing studies have been conducted by the FHWA using its ALF located at the Turner Fairbanks Highway Research Center (TFHRC) in MacLean, Virginia (Bonaquist and Mogawer, 1997) (Bonaquist, et al., 1998). The facility has been in operation since 1988 and uses a half-axle wheel assembly to apply loads 
varying from 45 to $100 \mathrm{kN}(10,000$ to $22,500 \mathrm{lbs})$ at a speed of $18.5 \mathrm{kph}(11.5 \mathrm{mph})$.

Traffic can be applied with or without wheel wander. The ALF is mobile and can be moved to various test sites in order to conduct testing on in-service pavements. The advantages of using accelerated pavement testing have been clearly established through the findings of various studies conducted at this facility.

A research study was started in 1993 was undertaken to verify some of the SHRP asphalt research program findings (Bonaquist et al., 1998). The main focus of the study was to investigate the effect of binder properties on rutting and fatigue performance of flexible pavements. The objectives were to: 1) "Confirm that the binder properties identified by the SHRP research as the determinants of pavement performance do significantly affect pavement performance;" 2) "verify the performance prediction capability of the proposed Superpave accelerated mixture tests"; and 3) "provide controlled loading performance data that can be used in the validation of the pavement performance models in the Superpave." This study made use of full-scale accelerated pavement tests, laboratory binder and mixture tests and the comparison of the expected and the actual pavement performance.

Twelve test lanes; each $4 \mathrm{~m}$ wide (13.2 ft) and $44 \mathrm{~m}(145 \mathrm{ft})$ long were tested.

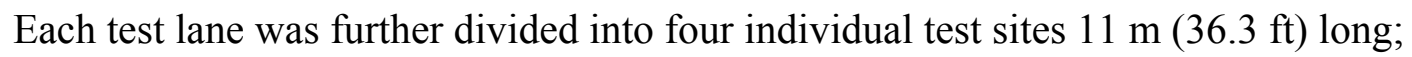
resulting in 48 individual test sites that were tested using a wide range of binders. The details of the binder grades, mixture types, and the pavement thickness are shown in the Table 2-4. The Superpave binder rutting parameter $\mathrm{G}^{*} / \sin \delta$ was validated using the ALF rutting performance and DSR data. Rutting performance data of the asphalt is shown in Table 2-5. 
Table 2-4 FHWA ALF Superpave Binder Validation Study Design (Bonaquist and Mogawer, 1997)

\begin{tabular}{|c|c|c|c|c|c|}
\hline $\begin{array}{c}\text { Lane } \\
\text { No. }\end{array}$ & $\begin{array}{c}\text { Binder } \\
\text { Grade }\end{array}$ & $\begin{array}{c}\text { Mixture } \\
\text { Type }\end{array}$ & $\begin{array}{c}\text { HMA Thickness } \\
(\mathrm{mm})\end{array}$ & $\begin{array}{c}\text { Base } \\
\text { Thickness (mm) }\end{array}$ & $\begin{array}{c}\text { Subgrade } \\
\text { Thickness mm) }\end{array}$ \\
\hline 1 & PG 58-34 & SM-3B & 102 & 559 & 610 \\
\hline 2 & PG 64-22 & SM-3B & 102 & 559 & 610 \\
\hline 3 & PG 58-34 & SM-3B & 204 & 457 & 610 \\
\hline 4 & PG 64-22 & SM-3B & 204 & 457 & 610 \\
\hline 5 & PG 58-28 & SM-3B & 204 & 457 & 610 \\
\hline 6 & PG 64-22 & SM-3B & 204 & 457 & 610 \\
\hline 7 & PG 82-22 & SM-3B & 204 & 457 & 610 \\
\hline 8 & PG 76-22 & SM-3B & 204 & 457 & 610 \\
\hline 9 & PG 58-34 & SM-3B & 204 & 457 & 610 \\
\hline 10 & PG 64-22 & SM-3B & 204 & 457 & 610 \\
\hline 11 & PG 58-34 & BM-3 & 204 & 457 & 610 \\
\hline 12 & PG 64-22 & BM-3 & 204 & 457 & 610 \\
\hline
\end{tabular}

As shown in Figure 2-7, the study reported that HMA rutting behavior could be characterized into two zones. At lower values of $\mathrm{G}^{*} / \mathrm{sin} \delta$, the rutting performance is very sensitive to binder grades and therefore the rutting parameter $\mathrm{G}^{*} / \sin \delta$ had a strong correlation with the rutting performance measured by the ALF and laboratory wheel testers. At higher values of $\mathrm{G}^{*} / \sin \delta$, rutting performance was less sensitive; indicating that for the modified mixtures, this parameter failed to characterize rutting resistance based on ALF or laboratory wheel testers. This study confirmed the need for additional binder and mixture characterization of modified binders. Mixtures with larger nominal maximum sizes showed improved rutting resistance as well.

A national pooled fund study (NPFS 176) (Stiady, 2000) (Hand et al., 2000) (Stiady et al., 2003) was conducted at Purdue University to validate the SHRP mixture specifications using accelerated pavement testing. The research study specifically focused on the validation of various aggregate specifications and volumetrics established by the Superpave mixture design method. The study addressed the effects of VMA, fine 
aggregate angularity (FAA), and gradation on the rutting performance of Superpave mixtures. The study consisted of APT, PURWheel, triaxial, and Superpave Shear Tests. HMA mixtures containing fine and coarse gradations with respect to Superpave restricted zone were investigated along with in-place density and the VMA. The experimental design of the study is shown in Table 2-6.

Table 2-5 Asphalt Rutting Performance (After Bonaquist and Mogawer, 1997)

\begin{tabular}{|c|c|c|c|c|c|c|c|c|c|c|c|c|}
\hline \multirow[b]{3}{*}{ Passes } & \multicolumn{12}{|c|}{ Asphalt Layer Rut Depth (mm) } \\
\hline & \multirow{2}{*}{$\begin{array}{l}\text { Lane 5 } \\
\text { Site 2 }\end{array}$} & \multicolumn{2}{|c|}{ Lane 7} & \multicolumn{2}{|c|}{ Lane 8} & \multicolumn{2}{|c|}{ Lane 9} & \multicolumn{2}{|c|}{ Lane 10} & \multicolumn{2}{|c|}{ Lane 11} & \multirow{2}{*}{$\frac{\text { Lane } 12}{\text { Site } 2}$} \\
\hline & & Site 1 & Site 2 & Site 1 & Site 2 & Site 1 & Site 2 & Site 1 & Site 2 & Site 1 & Site 2 & \\
\hline 1 & 0.2 & 0.8 & 0.2 & 1.4 & 0.2 & 0.2 & 0.2 & 1.9 & 0.6 & 0 & 0.3 & 0.5 \\
\hline 10 & 2.9 & 2.5 & 1.5 & 3.4 & 0.7 & 4.3 & 3.0 & 4.6 & 2.8 & 2.1 & 2.4 & 3.4 \\
\hline 100 & 6.3 & 6.3 & 3.5 & 5.6 & 2.2 & 10.1 & 9.6 & 8.8 & 8.0 & 5.4 & 5.7 & 5.8 \\
\hline 500 & 11.9 & 8.7 & 5.6 & 7.7 & 3.4 & 16 & 14.5 & 12.3 & 13.8 & 8.8 & 10.1 & 9.3 \\
\hline 1000 & 14.7 & 10.1 & 6.3 & 8.2 & 2.9 & 21.1 & 27.7 & 14.2 & 15.5 & 9.3 & 10.6 & 10.6 \\
\hline 1500 & - & - & - & - & - & 23.5 & - & - & - & - & - & - \\
\hline 2000 & 20.7 & - & - & - & - & $30.9^{b}$ & $36.8^{b}$ & - & - & - & 13.4 & - \\
\hline 3000 & 23.8 & - & - & - & - & & & 19.8 & - & 11.9 & 15.2 & 10.6 \\
\hline 4000 & $27.4^{b}$ & - & - & - & - & & & - & - & - & 16.9 & - \\
\hline 5000 & & 13.6 & 7.1 & 9.1 & 2.9 & & & 27.2 & 27.4 & 13.8 & 18.1 & 14.4 \\
\hline 7000 & & - & - & - & - & & & 24.9 & - & 15.7 & - & - \\
\hline 10000 & & 14.9 & 12.0 & 10.7 & 4.4 & & & $27.1^{b}$ & $36.3^{b}$ & 17.7 & 22.3 & 15.2 \\
\hline 15000 & & - & - & - & - & & & & & 20.8 & 23.6 & - \\
\hline 20000 & & - & - & - & - & & & & & 21.6 & 24.1 & - \\
\hline 24000 & & - & - & - & - & & & & & - & $24.9^{b}$ & - \\
\hline 25000 & & 17.0 & 14.3 & 11.3 & - & & & & & $23.4^{\mathrm{b}}$ & & 18.2 \\
\hline 35500 & & - & - & - & 5.8 & & & & & & & - \\
\hline 50000 & & 18.3 & 16.6 & 14.0 & - & & & & & & & 20.6 \\
\hline 60000 & & - & - & - & - & & & & & & & 20.9 \\
\hline 60500 & & - & - & - & 7.5 & & & & & & & - \\
\hline 75000 & & 18.6 & 17.0 & 15.3 & - & & & & & & & 21.2 \\
\hline 85500 & & - & - & - & 7.7 & & & & & & & 21.9 \\
\hline 100000 & & 19.1 & 16.9 & 16.2 & - & & & & & & & - \\
\hline 110500 & & & - & - & 7.6 & & & & & & & - \\
\hline 125000 & & $19.7^{\mathrm{b}}$ & 17.6 & $18.3^{b}$ & - & & & & & & & 22.9 \\
\hline 135500 & & & - & & 8.5 & & & & & & & - \\
\hline 150000 & & & 17.9 & & - & & & & & & & 23.5 \\
\hline 160500 & & & - & & 8.9 & & & & & & & - \\
\hline 175000 & & & 18.2 & & - & & & & & & & - \\
\hline 176735 & & & - & & - & & & & & & & 23.5 \\
\hline 185500 & & & - & & 9.2 & & & & & & & - \\
\hline 200000 & & & $18.1^{\mathrm{b}}$ & & - & & & & & & & $24.1^{b}$ \\
\hline 208805 & & & & & $9.2^{b}$ & & & & & & & \\
\hline \multicolumn{13}{|c|}{ Regression Model Parameters } \\
\hline $\bar{A}$ & 0.933 & 2.956 & 1.546 & 2.304 & 0.574 & 1.389 & 1.132 & 2.397 & 1.924 & 1.242 & 1.910 & 2.906 \\
\hline$\underline{B}$ & 0.406 & 0.166 & 0.248 & 0.170 & 0.227 & 0.398 & 0.459 & 0.268 & 0.296 & 0.289 & 0.258 & 0.176 \\
\hline
\end{tabular}




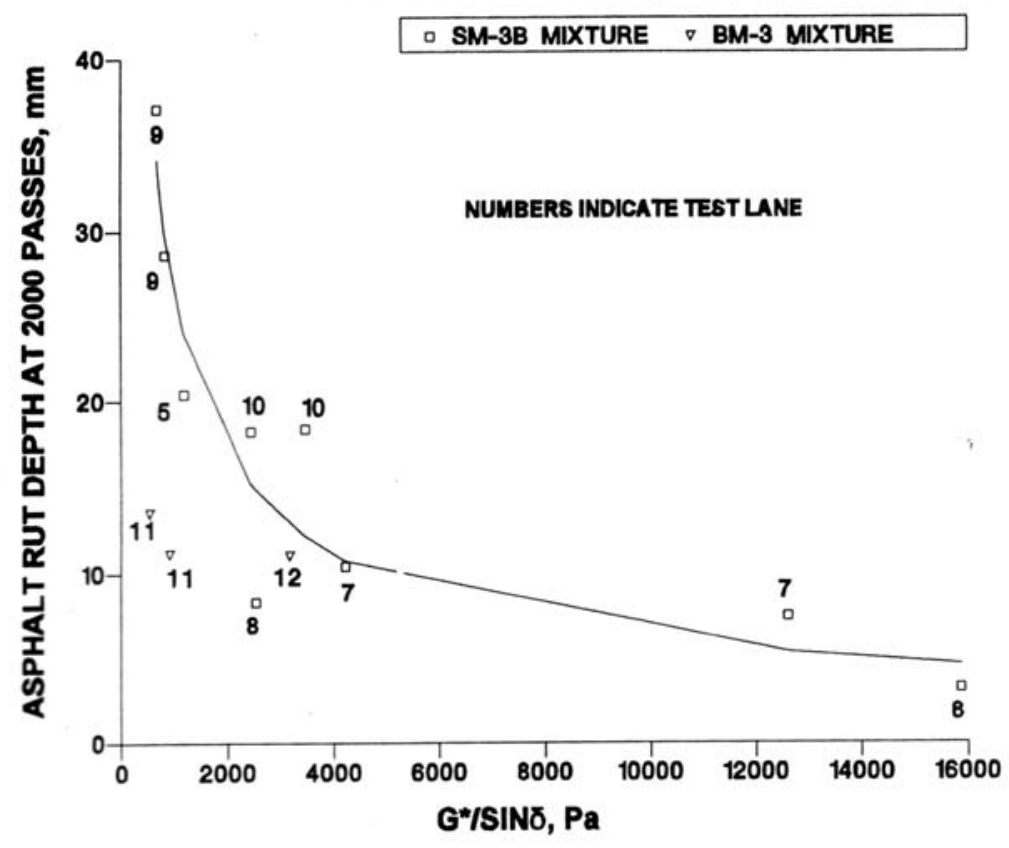

Figure 2-7 Effect of $\mathrm{G}^{*} / \sin \delta$ on Rutting (After Bonaquist and Mogawer, 1997)

Table 2-6 NPFS 176 Experimental Design (After Stiady, 2000)

\begin{tabular}{|c|c|c|c|c|c|c|c|c|c|c|c|c|c|c|c|c|c|}
\hline \multirow[t]{3}{*}{ FAA } & \multirow[t]{3}{*}{ Gradation } & \multicolumn{8}{|c|}{ 9.5 NMAS } & \multicolumn{8}{|c|}{$19 \mathrm{~mm}$ NMAS } \\
\hline & & \multicolumn{4}{|c|}{ Limestone } & \multicolumn{4}{|c|}{ Granite } & \multicolumn{4}{|c|}{ Limestone } & \multicolumn{4}{|c|}{ Granite } \\
\hline & & $\mathrm{A}$ & $\mathrm{P}$ & $\mathrm{T}$ & $\mathrm{M}$ & A & $\mathrm{P}$ & $\mathrm{T}$ & $\mathrm{M}$ & $\mathrm{A}$ & $\mathrm{P}$ & $\mathrm{T}$ & $\mathrm{M}$ & $\mathrm{A}$ & $\mathrm{P}$ & $\mathrm{T}$ & $\mathrm{M}$ \\
\hline \multirow[t]{2}{*}{39} & Above & & & & & & & & & & & & $\mathrm{X}$ & & & & \\
\hline & Below & & & & & & & & & & & & $X$ & & & & \\
\hline \multirow[t]{3}{*}{44} & Above & & $\mathrm{X}$ & & $\mathrm{X}$ & & & & & & & & $\mathrm{X}$ & & & & \\
\hline & Below & $\mathrm{X}$ & $\mathrm{X}$ & & $\mathrm{X}$ & $\mathrm{X}$ & $\mathrm{X}$ & $\mathrm{X}$ & $\mathrm{X}$ & $\mathrm{X}$ & $\mathrm{X}$ & & $\mathrm{X}$ & & $\mathrm{X}$ & $\mathrm{X}$ & $\mathrm{X}$ \\
\hline & Through & $\mathrm{X}$ & $\mathrm{X}$ & & $\mathrm{X}$ & $\mathrm{X}$ & $\mathrm{X}$ & $\mathrm{X}$ & $\mathrm{X}$ & & & & $\mathrm{X}$ & $\mathrm{X}$ & $\mathrm{X}$ & $\mathrm{X}$ & $\mathrm{X}$ \\
\hline \multirow[t]{3}{*}{50} & Above & & & & & & & & & & & $\mathrm{X}$ & $\mathrm{X}$ & & & & \\
\hline & Below & $\mathrm{X}$ & $\mathrm{X}$ & $\mathrm{X}$ & $\mathrm{X}$ & & $\mathrm{X}$ & $\mathrm{X}$ & $\mathrm{X}$ & $\mathrm{X}$ & $\mathrm{X}$ & $\mathrm{X}$ & $\mathrm{X}$ & $\mathrm{X}$ & $\mathrm{X}$ & $\mathrm{X}$ & $\mathrm{X}$ \\
\hline & Through & & $\mathrm{X}$ & $\mathrm{X}$ & $\mathrm{X}$ & $\mathrm{X}$ & $\mathrm{X}$ & $\mathrm{X}$ & $\mathrm{X}$ & $\mathrm{X}$ & $\mathrm{X}$ & $\mathrm{X}$ & $\mathrm{X}$ & & $\mathrm{X}$ & $\mathrm{X}$ & $X$ \\
\hline
\end{tabular}

$\mathrm{A}=$ INDOT/PURDUE APT

$\mathrm{P}=$ PURWheel

$\mathrm{T}=$ Dry Triaxial Tests

$\mathrm{M}=$ Mixture designs

$\mathrm{X}=$ Tests to Study Effects

The study reported that the effect of gradation on rutting performance is significant for some performance indicators (PURWheel and triaxial test) while not 
significant for others (APT). Mixtures plotting through the restricted zone exhibited higher rutting resistance than did fine- or coarse-graded mixtures in the PURWheel. Finegraded mixtures exhibited higher shear strength than did the coarse-graded mixtures and those with gradations passing through the restricted zone in the triaxial tests. The researchers noted that this result may indicate that the restricted zone alone might not be sufficient to optimize rutting performance. However, adequate rutting resistance can be achieved using the gradations plotted above, through, and below the restricted zone. The results also suggested that increasing FAA resulted in improved rutting performance.

NPFS 176 also compared the rutting performance of mixtures tested in the APT and PURWheel as shown in Figure 2-8 and 2-9. An excellent correlation existed between the rutting performances of the mixtures (9.75 $\mathrm{mm}$ and 19.0 NMAS) tested in the PURWheel and the APT for field-mixed, field compacted (FMFC) specimens. The data shows the ability of the PURWheel to correlate well with full-scale loading tests. The data also reported weaker correlations when field-mixed, laboratory compacted (FMLC) specimens or laboratory-mixed, laboratory compacted (LMLC) specimens were used for the PURWheel tests. This observation showed that compaction method and mixture preparation do effect the rutting performance of the mixtures tested at PURWheel.

A separate study conducted at Purdue using the APT to compare the rutting performance of various Superpave mixtures with that of a conventional mixture (Haddock and Ward, 1998). Two mixtures, a 19-mm intermediate and a 9.5-mm surface mixture were designed according to the Superpave volumetric design method. The conventional mixture was designed using the 75-blow Marshall method. Both APT PURWheel tests were conducted. Figures 2-10 and 2-11 show the APT rutting 
performances of the conventional and 19-mm Superpave mixtures. The Superpave mixtures outperformed the conventional mixture by 50 percent in the APT.

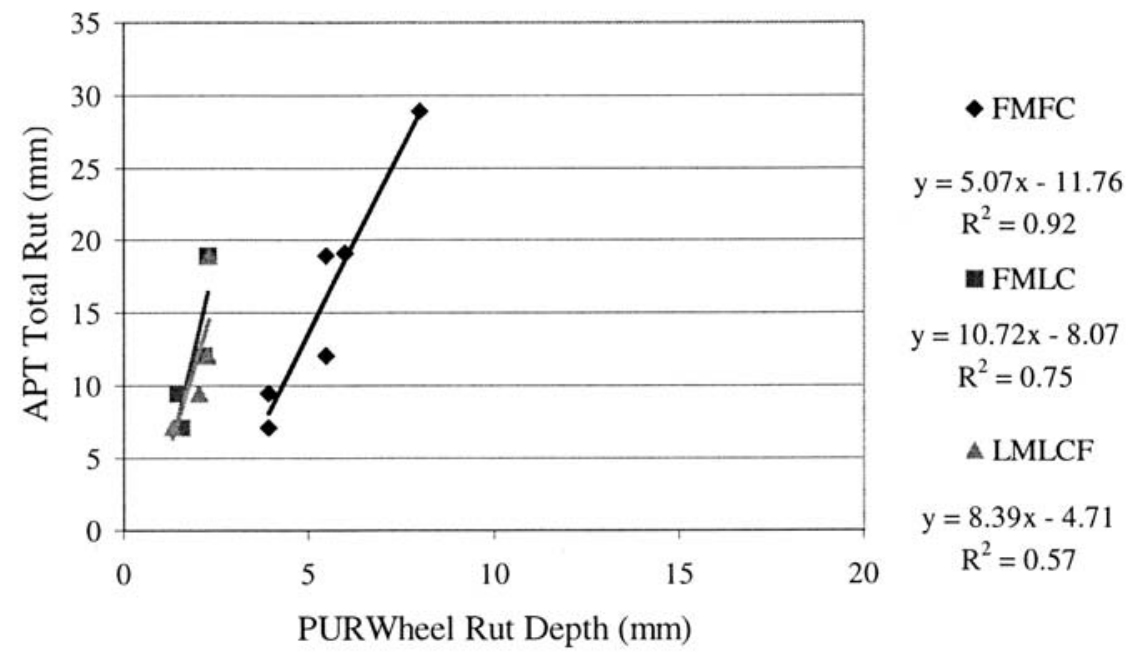

Figure 2-8 Relationship between APT Total Rut Depth and PURWheel Rut Depth (19 mm NMAS) (After Stiady, 2000)

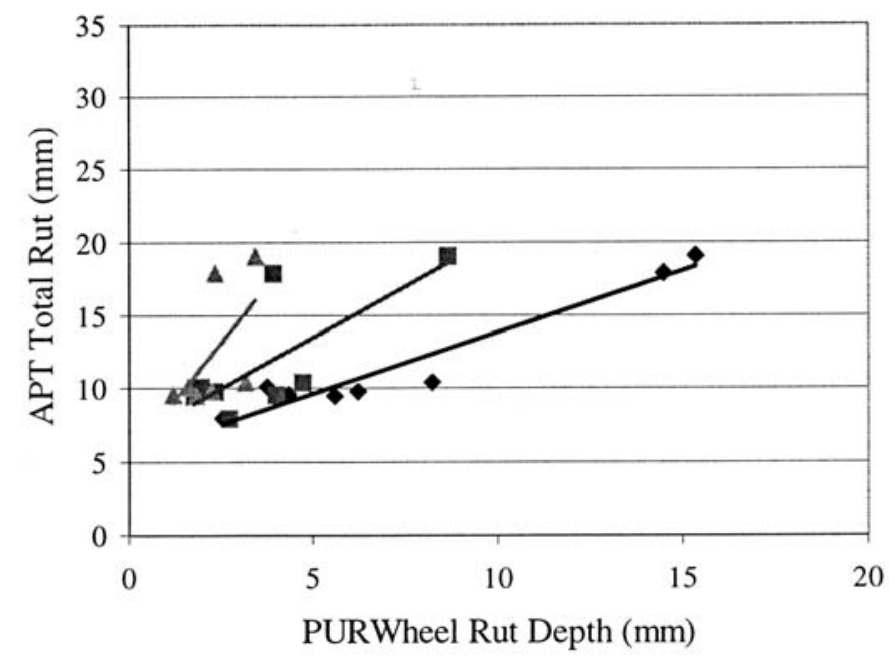

$$
\begin{aligned}
& \text { - FMFC } \\
& y=0.84 x+5.41 \\
& \mathrm{R}^{2}=0.93 \\
& \text { a FMLC } \\
& y=1.40 x+6.52 \\
& \mathrm{R}^{2}=0.55 \\
& \triangle \mathrm{LMLCF} \\
& y=3.14 x+5.21 \\
& \mathrm{R}^{2}=0.36
\end{aligned}
$$

Figure 2-9 Relationship between APT Total Rut Depth and PURWheel Rut Depth (9.5 mm NMAS) (After Stiady, 2000) 


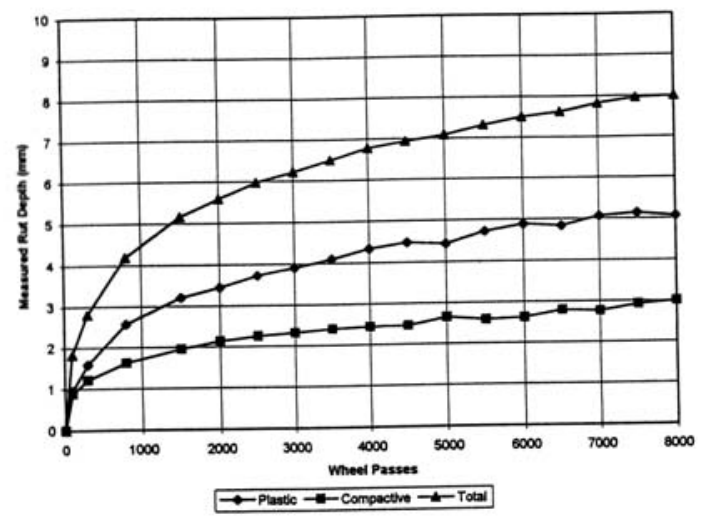

Figure 2-10 APT Rutting Performance of Conventional HMA Mixture Tested at $827 \mathrm{kPa}$ Tire Pressure (After Haddock and Ward, 1998)

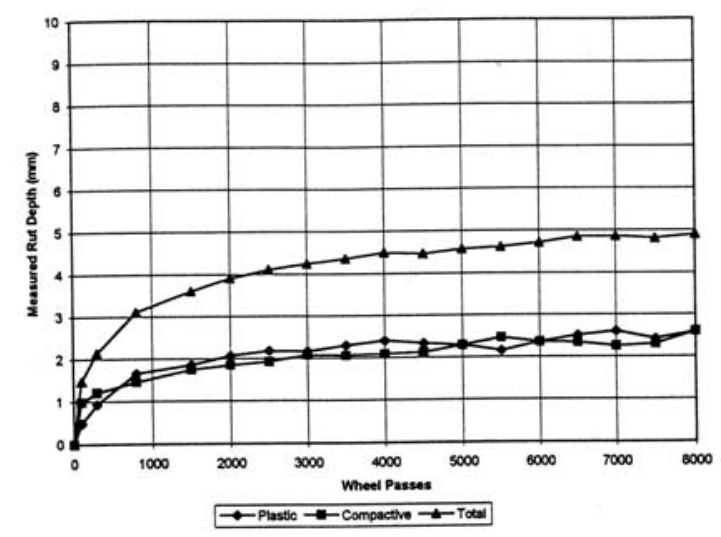

Figure 2- 11 APT Rutting Performance of a $19.0 \mathrm{~mm}$ Superpave HMA Mixture Testes at $827 \mathrm{kPa}$ (After Haddock and Ward, 1998)

Another study conducted at Purdue used the APT to investigate the effect of HMA mixture components on their rutting performance (Galal and White, 1999) (White et al., 1999). The study investigated the effect of aggregate type, percentage of crushed aggregate, and percentage of natural versus crushed sand and binder. A total of 32 Test sections were trafficked at 38C (100.4F). A standard $40 \mathrm{kN}(9,000 \mathrm{lbs})$ half axle was applied on the dual wheel assembly. The study reported that coarse aggregate type significantly affects HMA rutting and mixtures with crushed aggregates exhibited 50 
percent higher rutting resistance than mixtures containing rounded, uncrushed gravel aggregates. This study confirmed the importance of the aggregate type, shape and texture on the rutting performance of HMA mixtures.

The TxMLS is a full-scaled accelerated pavement tester designed and manufactured in Texas. It applies loads on test sections that are $11 \mathrm{~m}(36 \mathrm{ft})$ long. The significant difference between TxMLS and other ALF is that this device is loaded to six tandem axles, each weighing $150 \mathrm{kN}(34,000 \mathrm{lbs})$ while other APTs carry a-half single axles. The tire pressure is maintained at $690 \mathrm{kPa}(100 \mathrm{psi})$. The operating speed is $18 \mathrm{kph}$ (11 mph). A study was conducted to develop rutting performance models using TxMLS in Texas (Chen and Lin, 2001). The study involved in testing of TxMLS at three test sites; one in Victoria and two Jacksboro. The test section in Victoria consisted of a 75mm (3-in) surface layer, a 300-mm (12-in) lime treated gravel base and a 150-mm (6-in) lime stabilized subgrade. This section is $3 \mathrm{~m}(10 \mathrm{ft})$ wide and $12 \mathrm{~m}(40 \mathrm{ft})$ long. The inservice pavement test section in Jacksboro consisted of a 180-mm (7.2-in) asphalt concrete overlay on a 380-mm (15.2-in) base. The second site in Jacksboro was used to verify the model. The study recommended a rutting model that related rutting performance to surface deflections determined by the Falling Weight Deflection (FWD) tests, calculated stress at the top of base, and ESAL. The main drawback of this approach is that models are specific for the site on which the test data are collected.

The Louisiana Transportation Research Center houses an accelerated loading facility called the Louisiana ALF (LA ALF) in Port Allen, Louisiana. The LA ALF is 12 $\mathrm{m}(40 \mathrm{ft})$ long and travels along the pavement test section that are $61 \mathrm{~m}(201 \mathrm{ft})$ long by 4 $\mathrm{m}(13.2 \mathrm{ft})$ wide. Standard dual-tire, half axle loads ranging from $40 \mathrm{kN}(9,000 \mathrm{lbs})$ to 80 
$\mathrm{kN}(18,000 \mathrm{lbs})$ can be applied at a speed of $20 \mathrm{kph}(12.5 \mathrm{mph})$. One of the studies, conducted at this facility compared the rutting performance of a crumb rubber modified binder with that of the conventional binder (Mohammad et al., 2000). The researchers reported no significant improvement in the rutting performance for crumb rubber modified binder. This finding was consistent with the results obtained using laboratory characterization tests including the Superpave shear tester, indirect and axial creep tests, and resilient modulus.

The Kansas ALF was established in 1997 (Kansas ALF webpage) and consists of a test frame $12.8 \mathrm{~m}(42 \mathrm{ft})$ long that is attached to a standard axle (single or tandem). Varying the pressure in the suspension system produces the desired axle loading up to $180 \mathrm{kN}(40,000 \mathrm{lbs})$. The speed of the wheel assembly is $11.2 \mathrm{kph}(7 \mathrm{mph})$. The temperature of the pavement can be maintained between -10 to $45 \mathrm{C}$ (14 to $123 \mathrm{~F}$ ) using coils that circulate cool or hot ethylene glycol fluid and buried at three positions in the pavement structure. Tests can be conducted in two test pits each $2.4 \mathrm{~m}(8 \mathrm{ft})$ wide and $6.1 \mathrm{~m} \mathrm{(20} \mathrm{ft)} \mathrm{long.}$

The Kansas facility (Wu et al., 2000) has been used to validate the performance of two fine-graded Superpave HMA surface mixtures containing varying (15 to 30) percentages of the natural sand. A $150 \mathrm{kN}(34,000 \mathrm{lbs})$ tandem axle load was applied for 10,000 passes and after which varying loads ( 160 to $80 \mathrm{kN}$ ) and axles (tandem to single) were applied, while maintaining $620 \mathrm{kPa}(90 \mathrm{psi})$ tire pressure. The tests were conducted at 38C (98F). Both test sections exhibited severe rutting after the application of 80,000 passes. On the section with the higher amount of sand most of the rutting was due to shear flow. For the other section, most of the rutting was due to consolidation of HMA 
surface and/or other layers. HMA mixtures containing lower amounts of river sand had much better rutting resistance than mixtures containing larger amounts of river sand.

The Florida Department of Transportation (FDOT) initiated an accelerated pavement testing and research program in Gainesville, Florida in 2000 (Hugo and Epps, 2004). The objectives of this research program include "...validation of existing methods and materials, validation of Superpave mixture design methods and materials, long-range research, and trouble shooting type problem solving." The typical test section consists of eight linear tracks each $6 \mathrm{~m}(20 \mathrm{ft})$ long and $1.6 \mathrm{~m}(5.3 \mathrm{ft})$ wide. Accelerated loading is achieved using a HVS that travels at up to $12.8 \mathrm{kph}(8 \mathrm{mph})$ and is capable of hydraulically applying loads ranging from $32 \mathrm{kN}(7,000 \mathrm{lbs})$ to $204.5 \mathrm{kN}(45,000 \mathrm{lbs})$ on a super single or dual-tire assembly. The loads can be applied in one or two directions with or without a maximum wander of $0.8 \mathrm{~m}$ (30 in.). Both static and dynamic load in the sinusoidal form can be applied. The rutting measurements are taken using an automated transverse laser profiler and temperature can be controlled between room temperature and $70 \mathrm{C}(158 \mathrm{~F})$. Heating is provided using infrared heaters installed along both sides of the assembly.

A study conducted using the Florida HVS investigated the effect of binder type on HMA mixture rutting performance (Moseley et al., 2003). Two binders were used, a neat PG 67-22, and a modified PG 76-22. Testing was conducted at 50C (130F). The results showed that the use of modified binder increased rutting resistance by about 43 percent after the application of 175,000 wheels passes applied with $40 \mathrm{kN}(9,000 \mathrm{lbs})$ load. 


\subsubsection{Laboratory Test Devices}

Laboratory test devices used for the rutting evaluation apply scaled down loads at elevated temperatures resulting in accelerated damage. Such laboratory test devices include the Georgia Loaded Wheel Tester (GLWT) and its successor the Asphalt Pavement Analyzer (APA), Hamburg-Wheel Tester, French Rut Tester (FRT), and PURWheel.

Many studies were conducted using the FRT to establish standard specifications, and evaluate rutting performance of HMA mixtures in France (Brossead, et al., 1993). The studies investigated the effects of gradation, binder type and content, and temperature on rutting performance. The results indicated that reducing the sand content approximately 5 percent increases the rutting resistance measured in the FRT by 15 to 25 percent and that the use of larger amounts of coarse aggregates increases the HMA rutting resistance significantly. Gap-graded mixtures tended to reduce mixture stability resulting in lower rutting resistance. The modified binder increased the rutting resistance and exhibited only half the rutting as the neat binder mixtures.

As part of the SHRP validation of the Superpave binder properties, the $\mathrm{G}^{*} / \sin \delta$ rutting parameter was correlated with rutting performance measured in a laboratory wheel-tracking device at the University of Nottingham, United Kingdom. This study was executed under the contract A-003A (Leahy et al., 1994). The experimental study consisted of 16 binders each with different binder grades, two aggregate sources and two air void contents as shown in Table 2- 7. Rutting response was measured on $200 \mathrm{~mm}$ (8 in.) cylindrical specimens that had 5000 wheel passes applied while maintaining a contact pressure of $730 \mathrm{kPa}(105 \mathrm{psi})$. Both rut depth and rutting rate were measured. The results 
of the experiment are shown in Table 2-8. The results indicated that a poor relationship exists between the Superpave $\mathrm{G}^{*} / \sin \delta$ parameter and rutting performance as shown in Figure 2-12. It was concluded that the most of the rutting variation can be attributed to the aggregate properties and air void contents.

Table 2-7 Factor Levels of the SHRP Binder Validation Study (Leahy et al., 1994)

\begin{tabular}{|c|c|c|}
\hline $\begin{array}{c}\text { Binder } \\
\text { Grade }\end{array}$ & Aggregate Source & $\begin{array}{c}\text { Target Air } \\
\text { voids (Percent) }\end{array}$ \\
\hline $150 / 200$ & Crushed limestone & 4 \\
\hline AC-10 & Partially crushed gravel & 7 \\
\hline AC-8 & & \\
\hline AR-4000 & & \\
\hline AC-20 & & \\
\hline AR-4000 & & \\
\hline AC-30 & & \\
\hline $150 / 200$ & & \\
\hline AC-20 & & \\
\hline AC-5 & & \\
\hline AC-20 & & \\
\hline AC-20 & & \\
\hline AC-20 & & \\
\hline AC-20 & & \\
\hline AC-20 & & \\
\hline AR-4000 & & \\
\hline 16 binder grades & & \\
\hline
\end{tabular}

16 binder grades $\times 2$ aggregates $\times 2$ air voids $=64$ factor combinations 
Table 2-8 SHRP Binder Validation Study ANOVA Results (Leahy et al., 1994)

\begin{tabular}{|c|c|c|}
\hline \multirow{4}{*}{ Response } & $\begin{array}{c}\text { Factor or } \\
\text { interactions }\end{array}$ & $\begin{array}{c}\text { Proportional } \\
\text { Effects }\end{array}$ \\
\hline \multirow{4}{*}{ Rut Rate } & Binder & 26 \\
\cline { 2 - 3 } & Aggregate & 29 \\
\cline { 2 - 3 } & Air voids & 8 \\
\cline { 2 - 3 } & Binder $\times$ aggregate & 27 \\
\cline { 2 - 3 } & Error & 5 \\
\hline \multirow{4}{*}{ Rut Depth } & Binder & 31 \\
\cline { 2 - 3 } & Aggregate & 19 \\
\cline { 2 - 3 } & Air voids & 8 \\
\cline { 2 - 3 } & Binder $\times$ aggregate & 28 \\
\cline { 2 - 3 } & Error & 5 \\
\hline
\end{tabular}

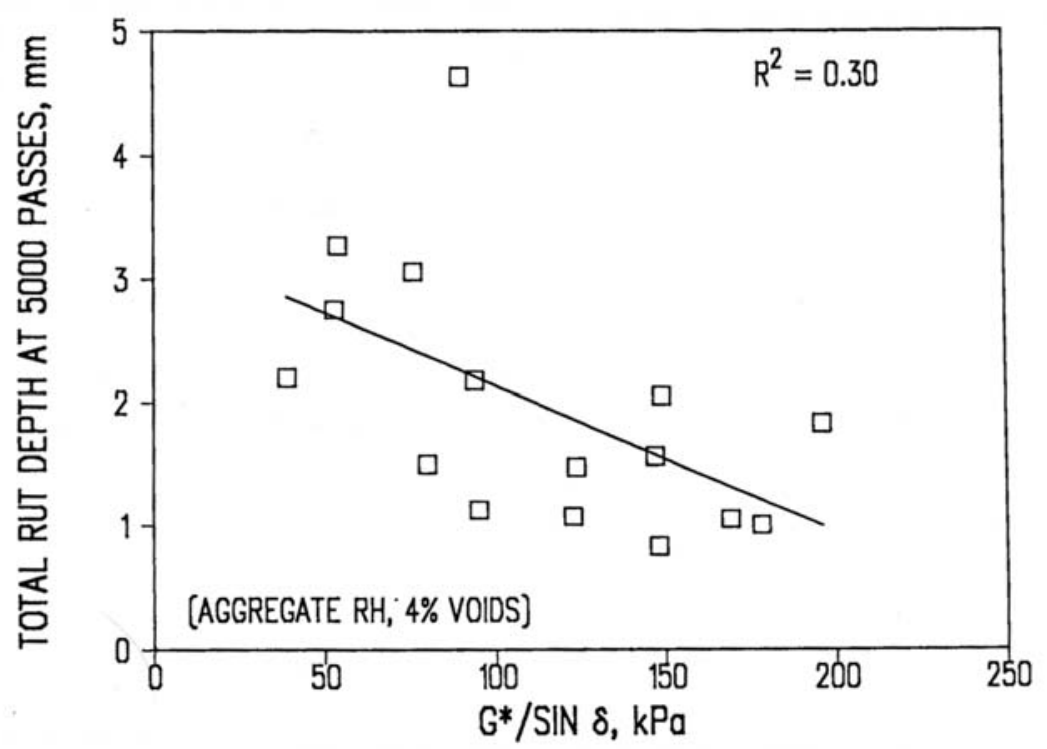

Figure 2-12 Relationship between $\mathrm{G}^{*} / \sin \delta$ and Total Rut Depth (After Leahy et al., 1994)

Though the study suggested that the effects of aggregate properties and air void contents are more pronounced on rutting performance than are the effects of $\mathrm{G}^{*} / \sin \delta$, several concerns related to the wheel-tracking device reduced the validity of the 
conclusions. The experimental error on the wheel-tracking device was large enough that it could have masked the effects of the binder properties. Further, the test temperature of the study was 40C (104F) and may not have been high enough to account for the viscous effects of binders. However, in a separate study, an excellent correlation between $\mathrm{G}^{*} / \mathrm{sin}$ $\delta$ and rut rate for modified binders was reported as shown in Figure 2-13 (Bouldin et al., 1994). Based on these conflicting results, it was decided that additional research was needed to evaluate the $\mathrm{G}^{*} / \sin \delta$ effects on rutting performance. The study also recommended that improvements to wheel-tracking tests were required to minimize experimental error and that tests should be conducted at higher contact stresses and temperatures.

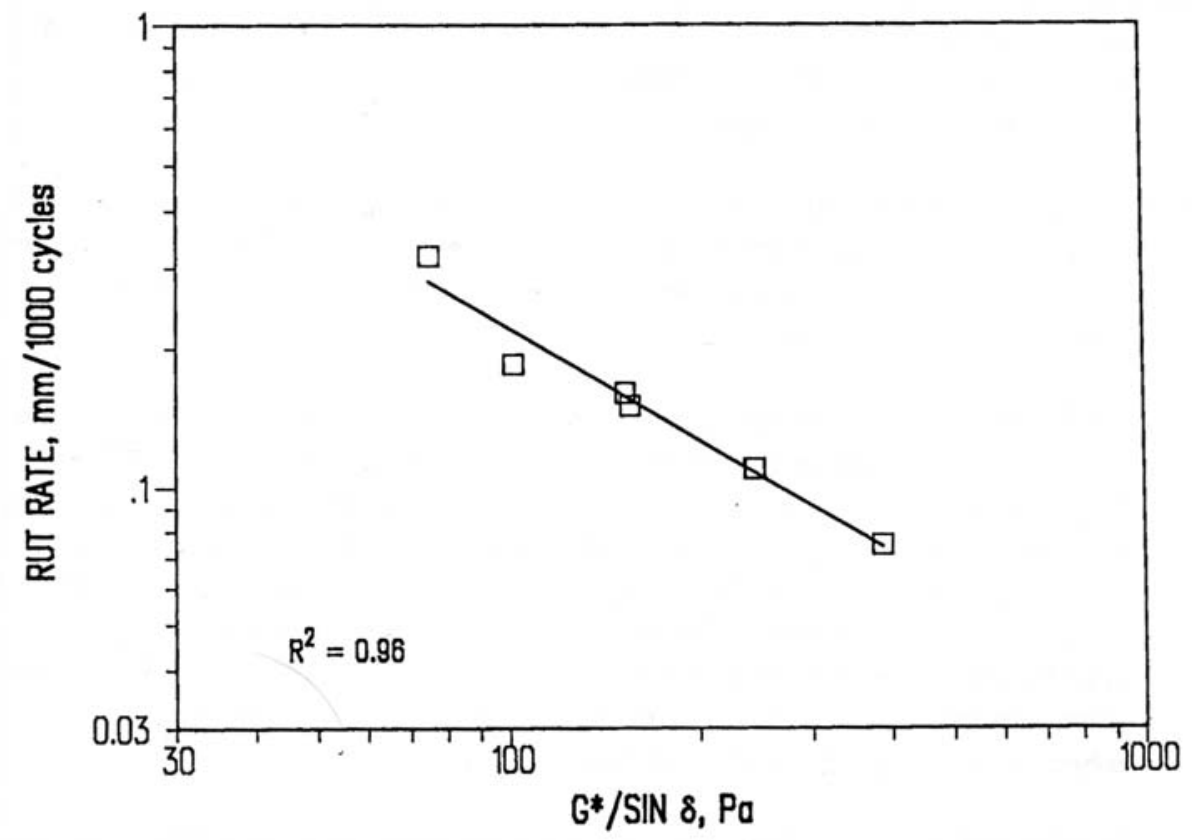

Figure 2-13 Relationship between $G^{*} / \sin \delta$ and Rut Rate (After Bouldin et al., 1994) 
In 1996, Brown and Gibb (1996) conducted tests in the Nottingham Pavement Test Facility to evaluate the capability of the repeated load, uniaxial compression test in ranking the rutting potential of HMA mixtures. The study included two aggregates (gravel and crushed granite) and two binders (neat and SBR modified) resulting in four aggregate binder combinations. The pavement structure consisted of $100 \mathrm{~mm}$ (4 in.) of HMA placed over $200 \mathrm{~mm}$ ( 8 in.) of crushed aggregate base supported by a silty-clay subgrade. The tests were conducted at a single wheel loading of $10 \mathrm{kN}(2,250 \mathrm{lbs})$ and $620 \mathrm{kPa}(90 \mathrm{psi})$ tire inflation pressure. The wheel speed was $4 \mathrm{kph}(2.5 \mathrm{mph})$ and loading was applied in one direction at 30C (86F) without wheel wander. HMA mixtures with modified binder showed improved rutting resistance. Further, the repeated load, uniaxial compression test ranking showed excellent agreement with the rutting performance observed on the small-scale test pavements.

When the WesTrack Forensic team (Brown et al., 1998, 2001) conducted a study to evaluate the rutting performances of coarse-graded HMA mixtures, four laboratory wheel testers were used: the APA, Hamburg, FRT, and PURWheel. The relationship between the APA and the test track was strong with a correlation coefficient $\left(\mathrm{R}^{2}\right)$ of 0.797. Tests were conducted in the APA according to NCHRP project 9-17 recommendations. Comparison of the Hamburg and test track yielded an $\mathrm{R}^{2}$ of 0.756 . The study suggested that the use of a steel wheel in the Hamburg generally results in severe rutting and that if an HMA mixture performs well in the Hamburg it should perform well in the field. However, HMA mixtures that fail in the Hamburg may also perform well in the field. The study cautioned against the potential rejection of an acceptable HMA mixture when tested using the Hamburg device. The FRT yielded an $\mathrm{R}^{2}$ 
of 0.694 and the forensic team concluded that the FRT can provide useful information when previous experience is available with the materials. The PURWheel yielded an $\mathrm{R}^{2}$ of 0.797 when an outlier was neglected as shown in Figure 2-14. The forensic team concluded that the PURWheel provides useful data where a previous relationship between in-service rutting performance and PURWheel rutting exists.

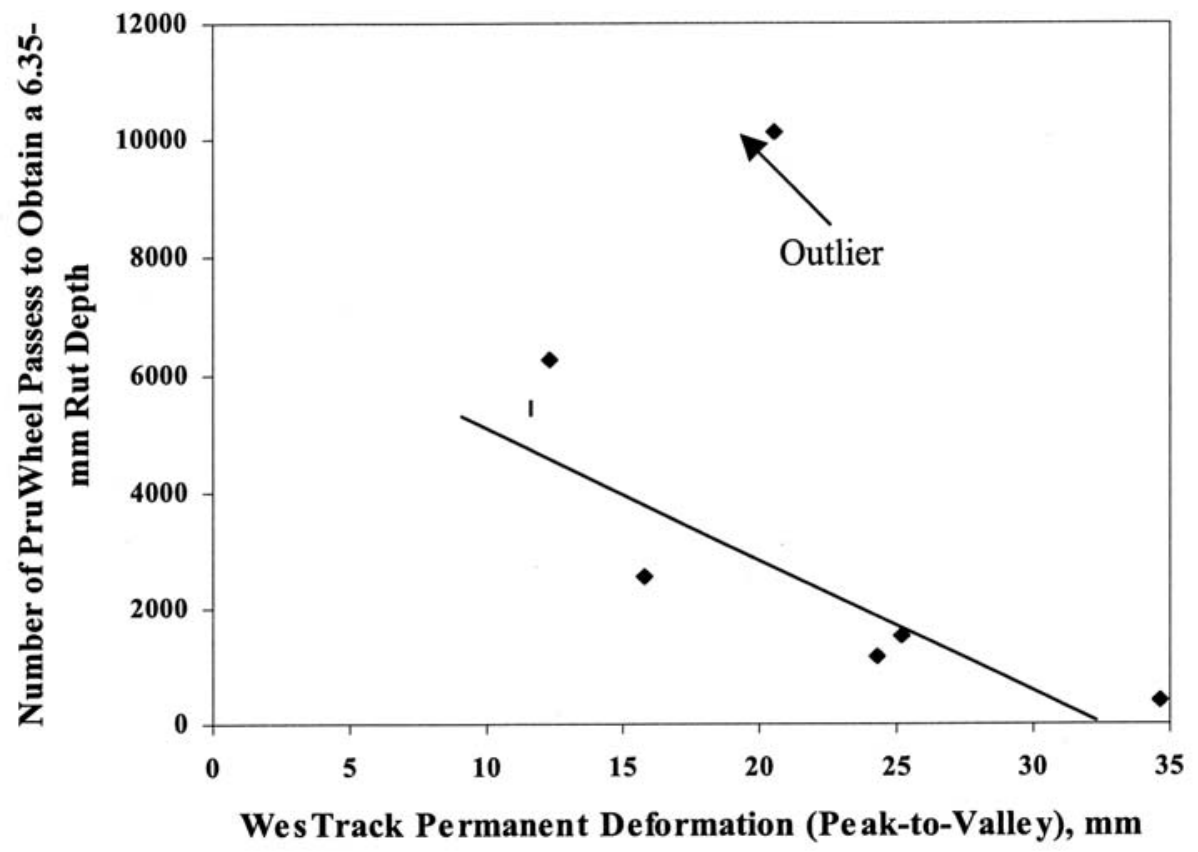

Figure 2-14 Relationship between WesTrack Rut Depth and Number of PURWheel Passes required for $6.35 \mathrm{~mm}$ Rut Depth (After Brown et al., 2001)

In addition, the APA performance was further analyzed by using mixtures placed on the Mn/Road project (Kandhal and Cooley, 2003). The rutting performance relationship between the APA and the actual in-service pavements at $\mathrm{Mn} / \mathrm{Road}$ yielded an $\mathrm{R}^{2}$ of 0.691 . However, when the data from both projects were combined rather weak correlation resulted. This was expected and can be explained by the difference in materials, climate, and loading conditions. 
Rutting data obtained from the FHWA ALF program was also used to evaluate laboratory rut testers (Bonaquist et al., 1998) (Stewart et al., 2000). The laboratory wheel tracking testers evaluated included the FRT, Hamburg, and GLWT. The rutting performance of the HMA mixtures at the ALF was compared with the laboratory measured rutting performance determined by the wheel testers. Tests were conducted at 60,50 , and 40C $(140,122$, and 104F) in the FRT, Hamburg, and GLWT, respectively. The comparison indicated that none of the laboratory testers provided significant advantage over the others for rutting prediction. The devices were not sensitive to NMAS in that increasing the NMAS did not increase rutting resistance as was observed in the ALF. However, rutting performance of laboratory rut testers was sensitive to widely varying binder properties. The study concluded that the geometry of the test specimens, stresses generated due to scale loading, and fixed temperature conditions do not simulate the rutting development of in-service pavements. However, the use of laboratory tests to evaluate the rutting potential of HMA mixtures is better than arbitrarily placing a volumetrically designed HMA mixture with no performance data.

As part of the same study, specimens were taken from the ALF facility prior to traffic application and tested in the PURWheel at 58C (136F). Based on the limited data generated, the PURWheel did show good correlation with ALF rutting performance $\left(\mathrm{R}^{2}=0.8\right)$ when all mixtures were included. It also identified the effects of the NMAS on the rutting performance, whereas the other laboratory testers did not. However, they were more sensitive to binder grades than was the PURWheel.

Many research studies were conducted to evaluate the potential of APA for rutting prediction (Kandhal and Mallick, 1999). Specifically, the sensitivity of the APA to the 
aggregate gradation and binder grades was investigated and a comparison made with the rutting performance of Superpave shear tester. Limestone, granite, and gravel aggregate were used to produce gradations above, through and below the Superpave restricted zone. The results indicated that performance of the APA is sensitive to aggregate gradation and binder grades and therefore can be used for the HMA rutting potential evaluation. Based on the test results, mixtures with gradations through the restricted zone exhibited higher rutting resistance than did coarse-graded HMA mixtures for limestone and granite aggregates. However, fine-graded mixtures showed the least resistance while coarsegraded mixtures showed high rutting resistance for mixtures made from gravel aggregates. PG 64-22 binder exhibited improved rutting performance over PG 58-22. The APA also showed a fair correlation $\left(\mathrm{R}^{2}\right.$ of 0.62$)$ with the repeated shear constant height test performed on Superpave shear tester as shown in Figure 2-15.

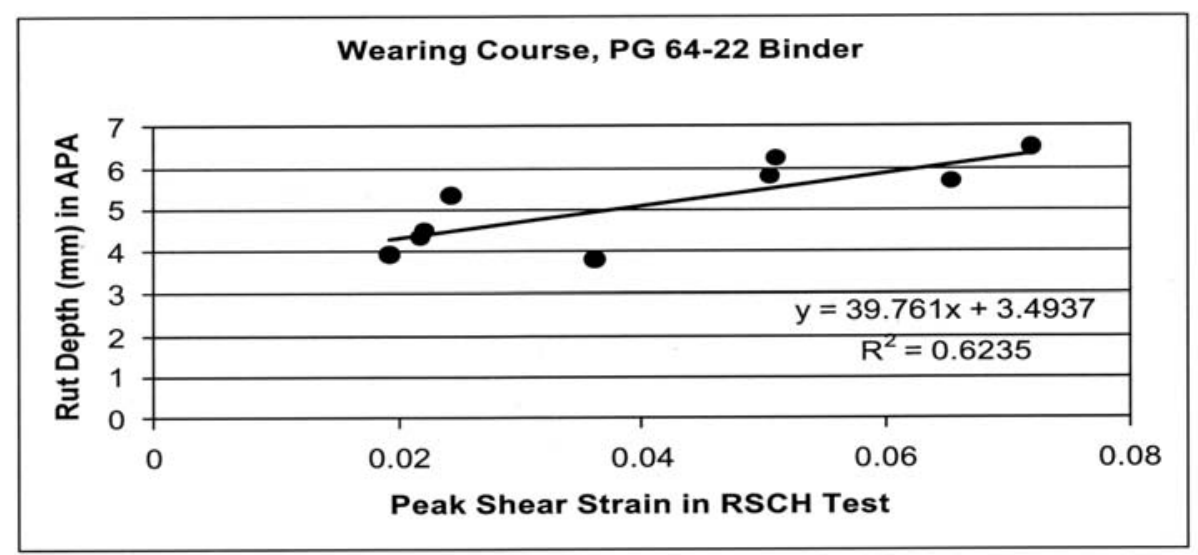

Figure 2-15 Relationship between Peak Strain in Repeated Shear Constant Height Test and Rut Depth in APA for Wearing Course with PG 64-22 Binder (After Kandhal and Mallick, 1999) 


\subsection{Modeling of HMA Mixtures and Pavements}

\subsubsection{Behavior of Asphalt Binder}

It is important to clearly understand the behavior of HMA mixtures in order to make improvements in their performance. HMA consists of aggregates and asphalt binder. The aggregates form a skeletal structure that resist permanent deformation under traffic loads while the binder acts to bond the structure together. It is apparent that the behavior of HMA is dependent on the behavior of its individual components, their proportioning, and their interaction (McGennis et al., 1995).

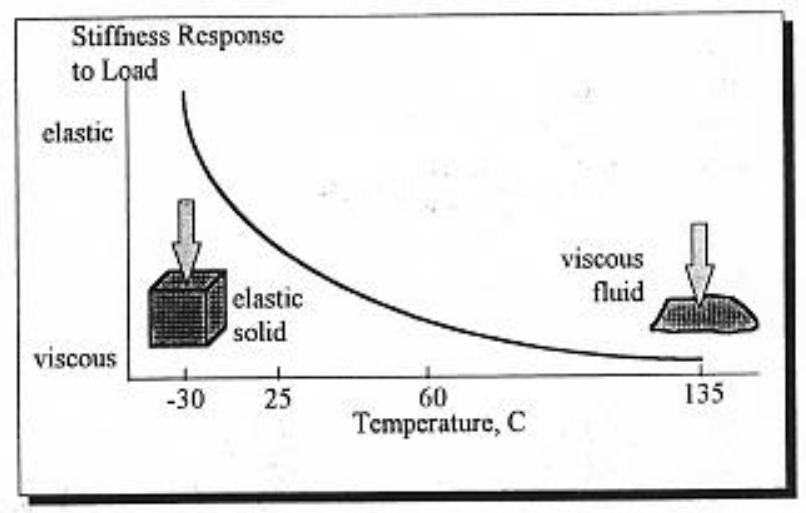

Figure 2-16 Visco-Elastic Behavior of Asphalt Binder (After McGennis et al., 1995)

The behavior of asphalt binder is quite complex. As shown in Figure 2-16, it behaves like a viscous material at high temperatures and an elastic material at low temperatures (McGennis et al., 1995). At intermediate temperatures, it behaves as both a viscous and an elastic material and so is called a visco-elastic material. The behavior of 
asphalt at slow loading rates is synonymous with high temperatures. Likewise, binder behaves elastically under fast loading rates just as if for low temperatures. Similar to most construction materials, its behavior also depends upon the applied stress. Asphalt binder is therefore known to be a material that is time, temperature and stress dependent.

\subsubsection{HMA Mixture Characterization}

Similar to asphalt binder, HMA is a time, temperature and stress dependent material. At higher temperatures and/or slow moving loads, it acts as a viscous material and begins to flow. This flow is irrecoverable and results in permanent deformation (rutting). In the literature, HMA mixtures are characterized as linear-elastic, plastic, visco-elastic and visco-plastic (creep) material.

Many researchers have used a linear-elastic model to characterize HMA mixtures (Kirkner et al., 1996). Many computer programs such as ELSYM5, BISAR, and others, have been developed using multi-layer, linear-elastic characterization. Two parameters are used to characterize linear-elastic materials, the modulus of elasticity and poison's ratio. Both can be determined using the resilient modulus or dynamic modulus tests. Linear-elastic models provide a simplified HMA material characterization as HMA mixture behavior is not linear-elastic as reported by Huang et al. (2000) and Seibi et al. (2000) among others.

A visco-elastic model captures the time, temperature, and stress dependent nature of HMA. One such model used by the Visco-elastic System (VESYS) program (FHWA, 1978) relates permanent strain to the elastic strain and visco-elatic behavior of the HMA material with the equation: 


$$
\varepsilon_{\mathrm{p}}=\varepsilon_{\mathrm{r}} \mathrm{N}^{\alpha} \mu / \alpha
$$

where,

$$
\begin{aligned}
& \varepsilon_{\mathrm{p}}=\text { percent permanent strain; } \\
& \varepsilon_{\mathrm{r}}=\text { percent resilient strain; } \\
& \mathrm{N}=\text { number of load applications; and } \\
& \mu \text { and } \alpha=\text { constants obtained from repeated load laboratory test. }
\end{aligned}
$$

Though the visco-elastic models are an improvement over the linear-elastic models, the visco-elastic models still fail to capture the important plastic response of a pavement.

Plastic models, such as the Drucker-Prager model, have been used to characterize HMA material (Olsson et al., 2000) (Huang et al., 2000). The linear Drucker-Prager model takes the form:

$$
\mathrm{F}=\mathrm{t}-\mathrm{p} \tan \beta-\mathrm{d}\left(\mathrm{w}_{\mathrm{p}}\right)=0
$$

where,

$$
t=\frac{q}{2}\left[1+\frac{1}{K}-\left(1-\frac{1}{K}\right)\left(\frac{r}{q}\right)^{3}\right]
$$

and,

$\mathrm{p}=$ equivalent pressure stress (psi);

$\mathrm{d}\left(\mathrm{w}_{\mathrm{p}}\right)=$ material parameter that includes plastic work hardening;

$\beta=$ slope of q Vs $p$ linear relationship;

$\mathrm{q}=$ Von Mises equivalent stress (psi);

$\mathrm{r}=$ third invariant of the deviatoric stress (psi); and

$\mathrm{K}=$ ratio of yield stresses in triaxial tension and compression. 
The parameters can be determined using triaxial tests with confining pressures that simulate in-situ conditions. This plastic model does not take into account the time dependent nature of HMA mixtures.

A nonlinear, elastic/viscous material was used to model HMA mixtures and develop damage models for their permanent deformation by Sousa et al. (1993). This model incorporated many of the observed characteristics demonstrated by HMA when subjected to rate-dependent loading at high temperatures. Among these are dilation under the shear loading, rate and temperature dependency, and increasing effective shear modulus under hydrostatic pressure conditions. The model used a number of threedimensional Maxwell elements connected in a parallel configuration. The Maxwell elements included a non-linear spring to represent the dilatancy and hardening behavior associated with aggregates and a dashpot to reflect the temperature and rate dependency behavior of the binder.

Seibi et al. (2000) developed an elastic, visco-plastic material model for HMA under high loading rates. It combines the rate dependent behavior with the DruckerPrager plastic model. Seibi used samples cored from the FHWA-ALF pavement to conduct uniaxial compression and pavement simulation tests. The simulation tests were conducted at various stress rates and three different temperatures. The uniaxial compression test data provided estimates for the material properties that were used as initial inputs in finite element modeling. However, actual pavements are subjected to multiaxial loading and the uniaxial material properties do not reflect the actual material behavior. The material characteristics were optimized by comparing the dynamic deflection predicted by the finite element model and the pavement simulation test data. 
ABAQUS and CONMIN were used for the FEM and optimization, respectively.

Optimized material properties were used as inputs to predict another loading history different form the one that was used for the calibration. Predicted deflections and the measured deflections showed excellent agreement as shown in Figure 2-17.

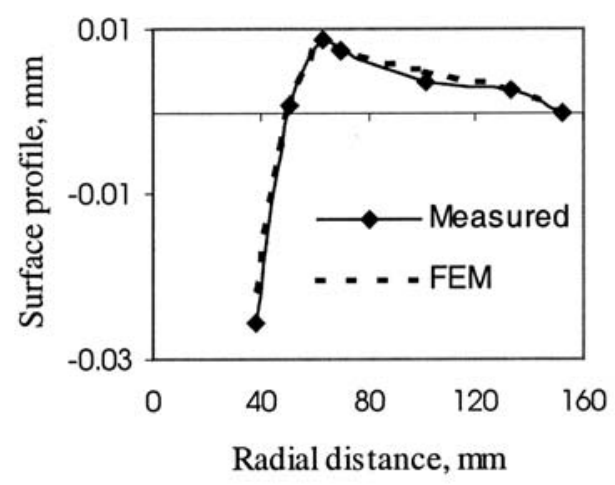

Figure 2-17 Predicted and Measured Surface Profiles of the Pavement Simulation Test $(\mathrm{t}=0.2 \mathrm{~s}$ and $\mathrm{T}=35 \mathrm{C})$ (After Seibi et al., 2000)

Studies conducted at Purdue University have used a visco-plastic (creep) model to characterize HMA mixtures (Huang, 1995) (Pan, 1997) (Hua, 2000) (Fang, 2000). It has been shown that a creep model successfully represents the time, temperature, and stress dependent nature of HMA. The creep model typically takes the form:

$$
\varepsilon_{C}=A \sigma^{n} t^{m}
$$

where,

$$
\begin{aligned}
& \varepsilon_{\mathrm{C}}=\text { creep strain; } \\
& \sigma=\text { stress (psi); } \\
& \mathrm{t}=\text { time (s); and }
\end{aligned}
$$

$\mathrm{A}$, and $\mathrm{m}=$ constants that are temperature dependent. 


\subsubsection{Modeling of HMA Rutting}

Generally, rutting modeling is performed using numerical methods such as FEM, direct-empirical regression methods that relate rutting to the mixture properties and traffic, or mechanistic-empirical methods.

Ljung and Sjoberg (2000) used a visco-elastic model for the HMA mixture and an elasto-plastic model for the unbound layers in their model. They compared the results of the finite element simulation with VEROAD; a multi-layer program, and results from the Lintrack test facility. ABAQUS was used for the FEM while the moving load was introduced using Matlab and Fortan. Finite element simulation yielded vertical and longitudinal strains that compared well with field measured data and analytical results estimated using VEROAD. The researchers concluded that FEM techniques provide more advantages with the potential for inclusion of different types of material models, boundary conditions, and load applications compared to VEROAD.

Yandell and Behzadi (1999) used elasto-plastic models and developed a numerical procedure called mechano-lattic analysis. They applied and validated this procedure using tests conducted in United States, South Africa, and Australia. They compared three methods to predict the rutting performances of $12 \mathrm{HMA}$ ALF test sections. They were modified CIRCLY, VESYS, and Mechano-lattice, elasto-plastic. CIRCLY is an elastic program capable of computing the elastic deformations at the desired depth in a pavement structure. Kinder's (1987) equation was used to estimate the permanent strain and plastic material parameters were either estimated using triaxial test data or obtained from Kinder's work. VESYS estimates a materials' permanent deformation using a visco-elastic material model. Repeated load, traxial tests were 
conducted at in-situ stress levels to estimate the model parameters required for the VESYS model. Mechano-lattice methods (Yandell, 1971, 1984) incorporated the directional movement of the wheel loads using three-dimensional finite element methods. Repeated triaxial loading tests were conducted to estimate the resilient modus and plastic properties. The results are shown in Figures 2-18 through 2-20. They indicate that in general, the modified CIRCLY method over estimates the rutting since it assumes that a perfect contact exists between the pavement layers, while VESYS underestimates the rutting. The mechano-lattice method resulted in the best prediction.

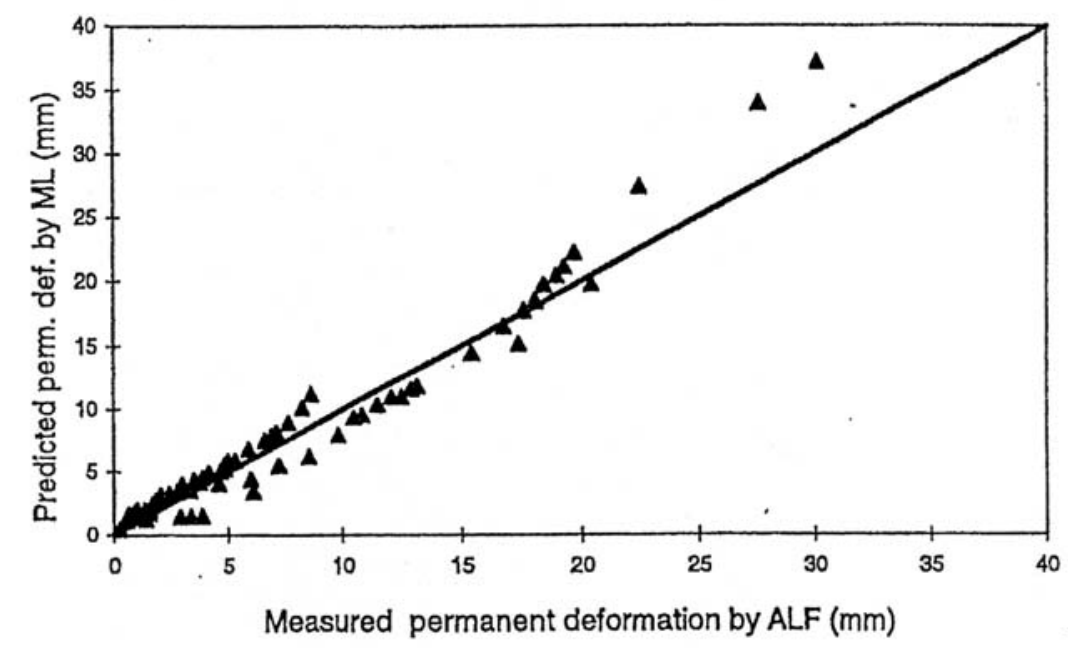

Figure 2-18 Relationship between Predicted Permanent Deformation by Mechano-lattice (ML) and Measured Values by ALF (After Yandell and Behzadi, 1999) 


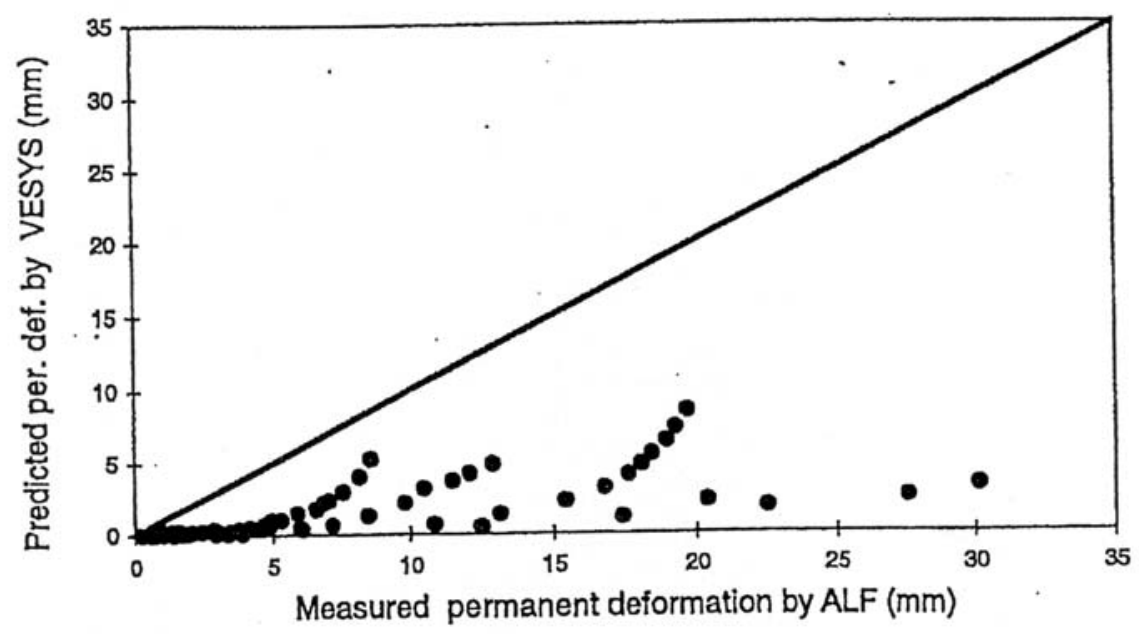

Figure 2-19 Relationship between Predicted Permanent Deformation by VESYS and Measured Values by ALF (After Yandell and Behzadi, 1999)

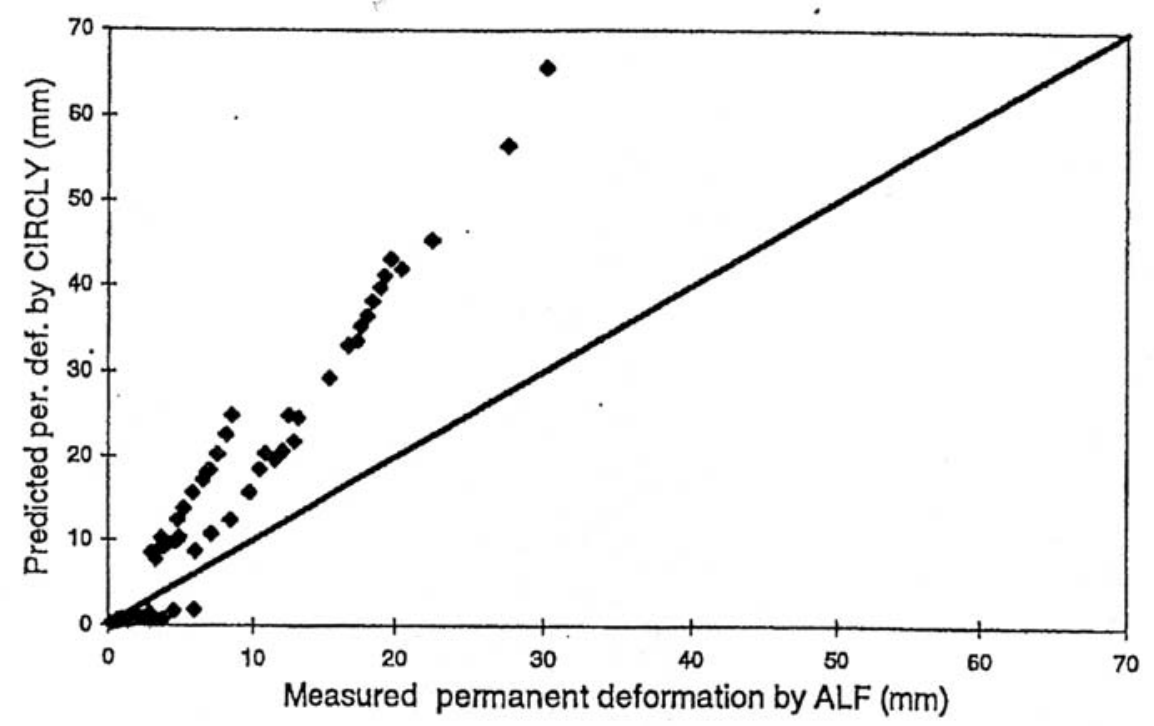

Figure 2-20 Relationship between Predicted Permanent Deformation by Modified CIRCLY and Measured Values by ALF (After Yandell and Behzadi, 1999) 
Huang et al. (2000) compared four types of finite element models used for pavement response prediction. They included two-dimensional static analysis with a linear-elastic materials model, three-dimensional dynamic analysis with a linear elastic model, three-dimensional dynamic analysis with a visco-plastic material model for HMA and elasto-plastic model for other layers, and three-dimensional rutting analysis with a visco-plastic creep model for HMA. The objective of the study was to "develop a 3-D finite element procedure using fundamental engineering properties acquired from mix characterization to simulate the pavement response under dynamic traffic load." The general purpose commercial finite element software ABAQUS was used. FWD-back calculation provided the elastic material parameters while uniaxial compressive tests conducted at varying strain rates provided the rate dependent visco-plastic material parameters for HMA. Triaxial tests were used to provide the parameters for the DruckerPrager model. One test lane of the LA ALF was used to calibrate the finite element modeling procedure. The study concluded that two-dimensional static analysis would not capture the dynamic loading effects and responses and that three-dimensional dynamic analysis are capable of predicting dynamic stress and strain responses. Further, they found that dynamic loading could be incorporated using the load functions of ABAQUS and permanent deformation could be predicted using a creep model and load function that includes the actual wheel wander in the three-dimensional dynamic finite element procedure. The study also compared the FEM predicted rut depth with average rutting observed on eight sections of the ALF as shown in Figure 2-21. 


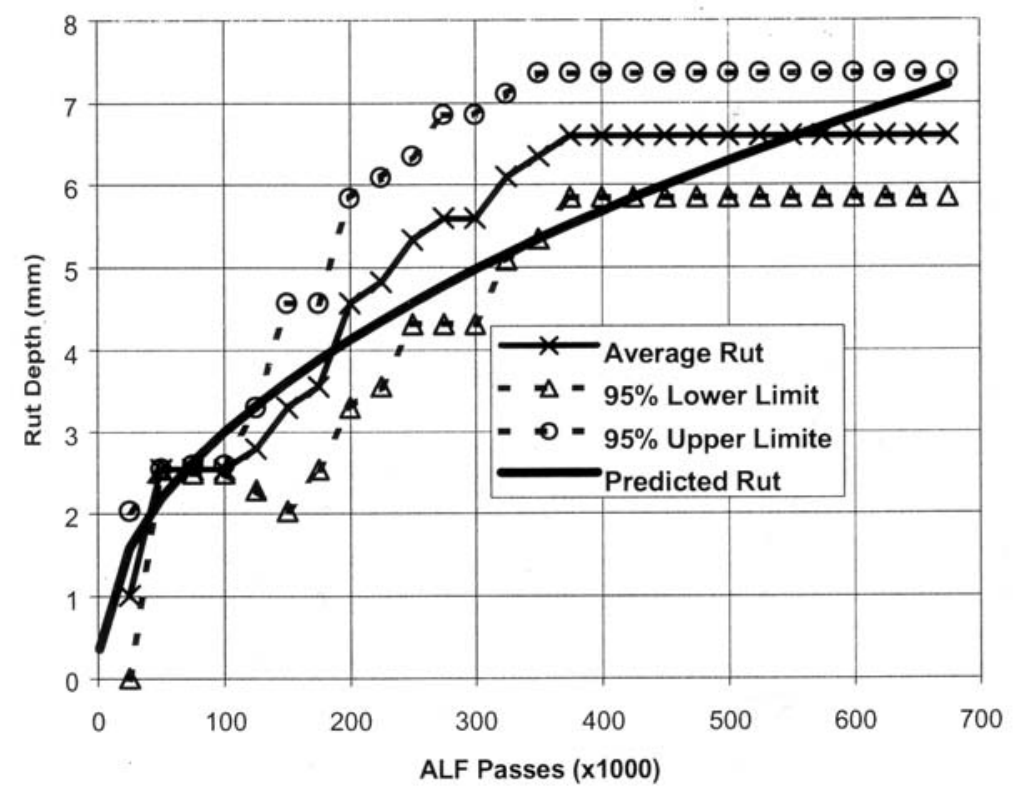

Figure 2-21 Predicted and Measured Rut Depth Development (After Huang et al., 2000)

Olsson et al. (2000) used a non-linear, visco-plastic material model to predict HMA rutting. This model combined creep (power law) with a conventional plastic model. The material parameters inputs for the FEM were obtained using laboratory experiments. ABAQUS was used for the modeling and the results were compared with Swedish design code data. It was assumed that the total strain consisted of elastic strain, plastic strain, creep strain and an environmental strain component. Elastic strain was computed using Hooks law. The Drucker-Prager yield surface defined the plastic strain while the power law defined creep strain. They found that this non-linear, visco-plastic model is appropriate for capturing HMA rutting phenomena and concluded that FEM could incorporate different material models applicable to the surface and the unbound layers. 
Zaghloul and White (1993) used a visco-elastic model to represent HMA and a plastic model for the unbound layers. Moving loads were simulated, but due to computational capability limits, only a few loading cycles were modeled. Typically, accelerated pavement testers need many loading cycles to develop rutting. Further, the contribution of visco-plasticity is not considered using a visco-elastic material model for the HMA.

Various other studies conducted at Purdue University have used a visco-plastic (creep) model to characterize the HMA mixtures (Huang, 1995) (Pan, 1997) (Hua, 2000) (Fang, 2000). Traffic loading was simplified using a total cumulative time based on wheel velocity and tire print measurements and applied as a static load. Studies by Hua (2000) and Fang (2000) incorporated wheel wander by distributing the loading time in the transverse direction. Creep material parameters were calculated by comparing the observed rutting development with the FEM predicted rutting development. An example of the material parameter determination is shown in Figure 2-22. Hua (2000) used a twodimensional finite element model to simulate in-service pavements using a full-depth HMA layer. Although, he calculated creep parameters, the results were not validated using in-service pavement data. Also, since the APT and PURWheel tests were conducted at a constant temperature, the effect of temperature on the rutting performance could not be investigated. 


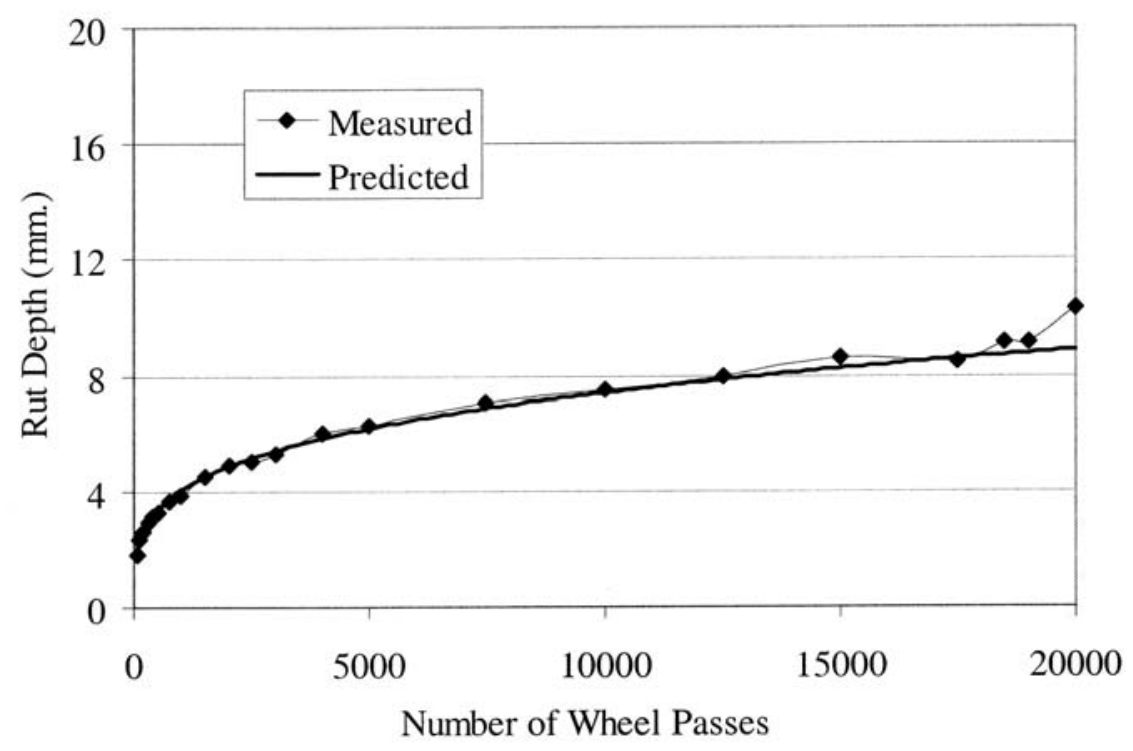

Figure 2-22 Fitted Curve of Single Wheel Path Rutting for 39 Below Mixture, Low density (After Hua, 2000)

Direct regression equations have also been used to relate HMA pavement rutting performance to mixture properties and traffic. Hand (1998) and Epps et al. (1998) developed a rutting regression model based on the WesTrack performance data using multiple linear regression techniques. The resulting equation is:

$$
\begin{aligned}
\operatorname{Ln} \mathrm{ESAL}_{10}=\mathrm{A}_{0}+ & \mathrm{A}_{1} \mathrm{P}_{\text {asp }}+\mathrm{A}_{2} \mathrm{~V}_{\text {air }}+\mathrm{A}_{3} \mathrm{P}_{\text {asp }}^{2}+\mathrm{A}_{4} \mathrm{~V}_{\text {air }}^{2}+\mathrm{A}_{5} \mathrm{P}_{\text {asp }} \mathrm{V}_{\text {air }} \\
& +\mathrm{A}_{6} \text { fines }+\mathrm{A}_{7} \text { fineplus }+\mathrm{A}_{8} \text { coarse }
\end{aligned}
$$

where,

$\mathrm{ESAL}_{10}=$ number of ESAL required to reach a $10 \mathrm{~mm}$ rut depth;

$\mathrm{P}_{\mathrm{asp}}=$ binder content $(\%)$;

$\mathrm{V}_{\mathrm{air}}=$ air voids content $(\%)$; and

$\mathrm{A}_{0}, \mathrm{~A}_{1}, \mathrm{~A}_{2}, \mathrm{~A}_{3}, \mathrm{~A}_{4}, \mathrm{~A}_{5}, \mathrm{~A}_{6}, \mathrm{~A}_{7}$, and $\mathrm{A}_{8}=$ regression coefficients. 
Fine, fine-plus, and coarse terms assume values of 1 for the type of mixture while other terms are 0 . These equations have limited use since they are only applicable to the conditions that existed at the test site from which they are developed and must be recalibrated for use in other climates.

As part of a national rutting study, Brown and Cross (1992) developed various rutting regression models. One relates the rutting rate to the aggregate properties and takes the form:

$$
\mathrm{P}=0.0031515-0.0000035(\mathrm{CF})-0.0005968(\mathrm{NAA})
$$

where,

$$
\begin{aligned}
& \mathrm{P}=\text { predicted rutting rate (in./square root } \mathrm{ESAL}) ; \\
& \mathrm{CF}=\text { percent coarse aggregate with } 2 \text { or more crushed faces (individual test); and } \\
& \mathrm{NAA}=\text { uncompacted air voids, method } \mathrm{A}(\%) .
\end{aligned}
$$

The study was initiated in 1987, and forty in-service pavement test sections from throughout the United States were sampled and thereby investigated a wide range of materials, climates, specifying agencies and construction practices. The study reported that most of the rutting developed in the top 75 to $100 \mathrm{~mm}$ (3 to 4 in.) of the pavement and therefore high quality materials should be used for HMA surface layers. The researchers also concluded that control of in-place air voids content above three percent is critical to prevent rutting due to premature instability. A rutting rate of 0.00023 inches per square root of ESAL delineated between good and poor rutting performance.

Mechanistic-empirical methods use the mechanical response of the pavement system (stress or strain) to predict the rutting performance. The material properties, loads and the geometry of the pavements are used as inputs and mechanical responses are 
typically determined using Burmister's layer theory (Kopperman et al., 1986). Empirical relationships that relate pavement response to rutting are established based on laboratory tests and in-service pavement performance data. These relationships are then used to predict and model the rutting performance. Vertical compressive strain at top of the subgrade was initially used to predict rutting based on the assumption that plastic strains are proportional to the elastic strains. This type of methodology predicts anticipated distress type and provides an opportunity for extrapolation of the limited laboratory and in-service data. ELSYM5 is one example of the mechanistic-empirical method and was developed at University of California, Berkley. This program is capable of handling twodimensional multiple wheels loads and uses a linear-elastic material model to characterize HMA. Assuming that plastic strains are proportional to the elastic strains, the rutting performance can be predicted.

Finn et al. (1986) used the AASHO rut depth data to develop two rutting models, depending on the depth of the layer in the pavement. These models are:

For HMA surface thickness $<150 \mathrm{~mm}$ (6 in.)

$$
\log (R R)=-5.617+4.343 \log d-0.167 \log (N 18)-1.118 \log \sigma_{c}
$$

For HMA surface thickness $>150 \mathrm{~mm}$ (6 in.)

$$
\log (R R)=-1.173+0.717 \log d-0.658 \log (N 18)+0.666 \log \sigma_{c}
$$

where,

$\mathrm{RR}=$ rate of rutting in (micro-inches/axle repetition);

$\mathrm{D}=$ surface deflection (mils under a load of $40 \mathrm{kN}(9,000 \mathrm{lbs})$ ); 
$\sigma_{\mathrm{c}}=$ vertical compressive stress at the HMA-base interface (psi); and

$\mathrm{N} 18=$ number of 18-kip single axle repetitions divided by 100,000 .

The Asphalt Institute (1991) also developed a rutting model:

$$
\mathrm{N}_{\mathrm{d}}=1.365 \times 10^{-9}\left(\varepsilon_{\mathrm{c}}\right)-^{4.447}
$$

where,

$\mathrm{N}_{\mathrm{d}}=$ number of axle loads required to reach a rutting of $12.5 \mathrm{~mm}(0.5 \mathrm{in}$.$) ; and$ $\varepsilon_{\mathrm{c}}=$ vertical compressive strain at the top of the subgrade.

Newcomb et al. (1999) developed a rutting model that was subsequently implemented in the ROADNET pavement design program.

$$
\mathrm{N}=5.5 \times 10^{15}\left(1 / \varepsilon_{\mathrm{v}}\right)^{3.949}
$$

where,

$\mathrm{N}=$ number of ESALs required to develop a $12.5 \mathrm{~mm}$ (0.5 in.) rut; and $\varepsilon_{\mathrm{v}}=$ vertical compressive strain at the top of the subgrade.

Finally, Williams et al. (1999) used the following relationship to establish a correlation between the permanent strain measured in the repeated load shear test at constant height and the number of load applications at the WestTrack:

$$
\varepsilon_{\mathrm{p}}=\mathrm{aN}^{\mathrm{b}}
$$

where,

$$
\begin{aligned}
& \varepsilon_{\mathrm{p}}=\text { percent permanent strain; } \\
& \mathrm{N}=\text { number of load applications, and } \\
& \mathrm{a} \text { and } \mathrm{b}=\text { modeling constants. }
\end{aligned}
$$




\section{CHAPTER 3 EXPERIMENTAL DESIGN AND RESEARCH APPROACH}

\subsection{Experimental Design}

For any research project it is essential that the appropriate design of experiment be made and implemented. The design should be made such that the effect of one variable is not masked by another important variable. Additionally, it should provide answers to the questions that are of interest to the sponsoring agency. In this study, INDOT was interested in validation of the Superpave mixture design method and in establishing rutting performance relationships among in-service pavements, the APT, and the PURWheel (Hand et al., 2000) (White, 1998). The experimental design attempts to address these issues; it is shown in Table 3-1.

The design includes three factors, each with two factor levels. The binder type factor has levels of neat and modified binder. The binder content factor includes optimum and optimum plus one-half percent, and the aggregate gradation factor incorporates fineand coarse-graded mixtures. The fine-graded mixtures plot above the maximum density line and Superpave restricted zone while the coarse-graded mixtures plot below these.

As shown in Table 3-1, each of the resulting test mixtures was placed on the NCAT test track. INDOT sponsored two sections (N3, and N4) while ALDOT supported four (N5, N6, N7, and N8). The FHWA funded two sections (N9, and N10) while the remaining two sections (N1, and N2) were paid for by the track project. 
Table 3-1 Experimental Design

\begin{tabular}{|c|c|c|c|}
\hline \multirow{2}{*}{ Binder } & Binder & \multicolumn{2}{|c|}{ NCAT/APT Section } \\
\cline { 3 - 4 } & Content (\%) & Fine-graded & Coarse-graded \\
\hline $67-22$ & Optimum & N4 & N6 \\
\cline { 2 - 4 } Neat & Optimum+0.5 & N3 & N5 \\
\hline $76-22$ & Optimum & N1 & N9 \\
\cline { 2 - 4 } SBS & Optimum+0.5 & N2 & N10 \\
\hline $76-22$ & Optimum & -1 & N8 \\
\cline { 2 - 4 } SBR & Optimum+0.5 & $-{ }^{-}$ & N7 $^{2}$ \\
\hline
\end{tabular}

${ }^{1}$ These mixtures were not included in the original study.

${ }^{2}$ These mixtures were not duplicated in the APT due to a lack of materials.

\section{$\underline{\text { 3.2 Materials }}$}

\subsubsection{Aggregates}

It was originally planned that Indiana limestone and blast furnace slag aggregates would be transported to the NCAT track site and used in mixture production for the Indiana sections (White, 1998). However, it was more practical to use aggregates from Alabama during track construction. Sufficient quantities of these aggregates were then transported to a local hot-mix plant in Lafayette, Indiana and used in the construction of the APT test sections. Table 3-2 shows the stockpile gradations of the three aggregates that were blended to meet target mixture gradations. The aggregates were tested and were found to meet the applicable INDOT aggregate requirements for a 10 million ESAL design.

\subsubsection{Asphalt Binders}

The three asphalt binders in the study were an unmodified (neat) PG 67-22, a Styrene Butadiene Styrene (SBS) modified PG 76-22, and a Styrene Butadiene Rubber 
(SBR) modified PG 76-22 binder. Each of the binders was shipped from the manufacturer to both the track and to the hot-mix plant in Lafayette. Superpave binder grade verification tests were performed by NCAT personnel and to verify that they did comply with applicable INDOT specifications.

Table 3-2 Stockpile Gradations

\begin{tabular}{|c|c|c|c|}
\hline \multirow{2}{*}{ Sieve Size } & $\begin{array}{c}|c| \\
\text { Limestone 892 } \\
\text { (\% Passing) }\end{array}$ & $\begin{array}{c}\text { Slag 78 } \\
\text { (\% Passing) }\end{array}$ & $\begin{array}{c}\text { Slag 8910 } \\
\text { (\% Passing) }\end{array}$ \\
\hline $3 / 4$ & 100 & 100 & 100 \\
\hline $1 / 2$ & 100 & 99.2 & 100 \\
\hline $3 / 8$ & 100 & 67.0 & 100 \\
\hline No 4 & 99.7 & 7.2 & 93.2 \\
\hline No 8 & 90.6 & 3.2 & 68.4 \\
\hline No 16 & 61.6 & 1.8 & 52.4 \\
\hline No 32 & 39.8 & 0.0 & 36.5 \\
\hline No 50 & 22.6 & 0.0 & 20.5 \\
\hline No 100 & 9.4 & 0.0 & 14.4 \\
\hline No 200 & 4.5 & 0.0 & 8.2 \\
\hline
\end{tabular}

\subsection{Mixture Design}

Fine and coarse gradations were obtained by blending stockpiles of limestone and blast furnace slag as shown in Table 3-3. NCAT personnel performed the HMA mixture designs using the Superpave volumetric mixture design method. Specimens for the design procedure were compacted with 100 gyrations of the SGC. The optimum binder content was chosen at 4 percent air voids. The mixture design data are shown in Tables 3-4 and 3-5. Five designs were completed to determine optimum binder content and the remaining five mixtures were produced by adding one-half percent more binder to each 
of these. As shown in Table 3-4, the design air voids and VMA are given only for designed mixtures.

Table 3-3 Details of the Mixture Type and Stockpile Percentages (Hand et al., 2000)

\begin{tabular}{|c|c|c|c|c|c|}
\hline \multirow[b]{4}{*}{ Mixture Type } & \multirow{4}{*}{$\begin{array}{l}\text { Number of } \\
\text { Test Sections }\end{array}$} & \multicolumn{4}{|c|}{ Material Type } \\
\hline & & \multirow{2}{*}{\multicolumn{2}{|c|}{$\begin{array}{c}\text { Blast Furnace Slag } \\
\text { Stockpile Percentages }\end{array}$}} & \multicolumn{2}{|c|}{ Limestone } \\
\hline & & & & Stockp & tages \\
\hline & & \# 78 & \# 8910 & \# 820 & $\# 892$ \\
\hline Fine & 4 & 31 & 34 & 0 & 35.0 \\
\hline Coarse & 6 & 47.5 & 17.5 & 35.0 & 0.0 \\
\hline
\end{tabular}

Table 3-4 Laboratory Mixture Design Data

\begin{tabular}{|c|c|c|c|c|c|c|c|}
\hline $\begin{array}{c}\text { NCAT } \\
\text { Section }\end{array}$ & $\begin{array}{c}\text { Design } \\
\text { Method }\end{array}$ & $\begin{array}{c}\text { Compactive } \\
\text { Effort } \\
\text { (gyrations) }\end{array}$ & $\begin{array}{c}\text { Binder } \\
\text { Type }\end{array}$ & $\begin{array}{c}\text { Binder } \\
\text { Content } \\
(\%)\end{array}$ & $\begin{array}{c}\text { Gradation } \\
\text { Type }\end{array}$ & $\begin{array}{c}\text { Air Voids } \\
(\%)\end{array}$ & $\begin{array}{c}\text { VMA } \\
(\%)\end{array}$ \\
\hline N1 & Superpave & 100 & SBS & 7.5 & ARZ & 4.0 & 15.8 \\
\hline N2 & Superpave & 100 & SBS & 8.0 & ARZ & - & - \\
\hline N3 & Superpave & 100 & Neat & 8.4 & ARZ & - & - \\
\hline N4 & Superpave & 100 & Neat & 7.9 & ARZ & 4.0 & 16.5 \\
\hline N5 & Superpave & 100 & Neat & 8.2 & BRZ & - & - \\
\hline N6 & Superpave & 100 & Neat & 7.7 & BRZ & 4.0 & 14.8 \\
\hline N7 & Superpave & 100 & SBR & & BRZ & - & - \\
\hline N8 & Superpave & 100 & SBR & 7.7 & BRZ & 4.0 & 15.1 \\
\hline N9 & Superpave & 100 & SBS & 7.3 & BRZ & 4.0 & 14.8 \\
\hline N10 & Superpave & 100 & SBS & 7.8 & BRZ & - & - \\
\hline
\end{tabular}

$\mathrm{ARZ}=$ Above restricted zone (Fine-graded) $\mathrm{BRZ}=$ Below restricted zone (Coarse-graded)

- Not a designed mixture. One-half percent binder content was added for the corresponding design mixture. 
Table 3-5 Mixture Design Gradations

\begin{tabular}{|c|c|c|c|c|c|}
\hline \multirow{2}{*}{$\begin{array}{c}\text { Sieve } \\
\text { Size }\end{array}$} & $\begin{array}{c}\text { N1 } \\
\text { (\% Passing) }\end{array}$ & $\begin{array}{c}\text { N4 } \\
\text { (\% Passing) }\end{array}$ & $\begin{array}{c}\text { N6 } \\
\text { (\% Passing) }\end{array}$ & $\begin{array}{c}\text { N8 } \\
\text { (\% Passing) }\end{array}$ & $\begin{array}{c}\text { N9 } \\
\text { (\% Passing) }\end{array}$ \\
\hline $3 / 4$ & 100 & 100 & 100 & 100 & 100 \\
\hline $1 / 2$ & 99.8 & 99.8 & 99.6 & 99.6 & 99.6 \\
\hline $3 / 8$ & 89.8 & 89.8 & 84.3 & 84.3 & 84.3 \\
\hline No 4 & 68.8 & 68.8 & 51.9 & 51.9 & 51.9 \\
\hline No 8 & 56.1 & 56.1 & 36.3 & 36.3 & 36.3 \\
\hline No 16 & 40.3 & 40.3 & 25.4 & 25.4 & 25.4 \\
\hline No 32 & 26.9 & 26.9 & 17.1 & 17.1 & 17.1 \\
\hline No 50 & 15.7 & 15.7 & 11.4 & 11.4 & 11.4 \\
\hline No 100 & 9.1 & 9.1 & 8.6 & 8.6 & 8.6 \\
\hline No 200 & 5.3 & 5.3 & 6.5 & 6.5 & 6.5 \\
\hline
\end{tabular}

\subsection{Research Approach}

The research consisted of HMA mixture testing at the NCAT test track, and in the APT and PURWheel. The ten HMA mixtures shown in Table 3-1 were initially produced and placed on the NCAT test track and subjected to 10 million ESAL in approximately two years time using four tractor-trailer combinations operating at $72 \mathrm{kph}(45 \mathrm{mph})$. Performance data was collected and included air and pavement temperatures, rut depths, moisture, and traffic. The rutting performance data from the test track sections provide validation information for the Superpave mixture design method.

Eight of the ten mixtures placed on the test track were also produced and placed in the APT. The two remaining mixtures were not duplicated in the APT due to a lack of materials. The APT sections were built following typical paving procedures. The sections were then trafficked with dual wheel assembly loaded to $40 \mathrm{kN}$ (9,000 lbs) and rutting data obtained. APT tests were primarily conducted at 50C (122F). To study the effects of 
temperature on rutting, tests were also conducted in the APT at 35C (86F) as well. Due to time and budget constraints only three mixtures were tested at both temperatures.

PURWheel testing was also completed during the project. Slabs from the test track and APT sections were tested in the PURWheel. The test track specimens were cut from the inside lane of the track and thus had no traffic applied prior to PURWheel testing. The test specimens taken from the APT sections were obtained prior to traffic application as well. PURWheel specimens were also fabricated in the laboratory using the linear compactor. This task was performed to compare the linear laboratory compaction method with the typical compaction methods used in highway pavement construction. These laboratory mixed, laboratory compacted (LMLC) specimens had the same binder content, aggregate gradation, and air voids of the test track mixtures. For each mixture, four replicates were produced and tested in the PURWheel at 50C (122F).

HMA is a time, stress and temperature dependent material and any efforts to characterize the material must account for these variables. A creep model was therefore selected and used to characterize the HMA mixtures. FEM was used to determine material parameters for the creep model and these used as inputs into the track model. FEM predicted data was compared with the measured data to establish rutting performance relationships between the test track, the APT, and the PURWheel. 


\section{CHAPTER 4 NCAT TEST TRACK}

\subsection{Overview}

The NCAT test track is located near Opelika, Alabama and is $2744 \mathrm{~m} \mathrm{(9000} \mathrm{ft)}$ long, including two tangents with a combined length of 1829 m (6000 ft) (White, 1998). Track construction began in the fall of 1998 and was completed in the summer of 2000. It was built and operated as a pooled fund study with the ALDOT serving as the host state and eight other states participating along with the FHWA. The eight other participating states were Indiana, Florida, Georgia, South Carolina, North Carolina, Tennessee, Mississippi, and Oklahoma. FHWA-Region 4 served as the coordinator of the study.

The test track includes 46 test sections, 26 of which are in the tangents and 20 on the curves. The curve sections were originally intended to be constructed continuously with the same HMA mixture (using gravel aggregates) and not included in the research. However, that decision was changed due to the fact that the same traffic would be applied to the curves as to the tangents and additional sections could be constructed with little increase in cost (Brown and Powell, 2001) (Powell, 2001) (Brown et al., 2002). The tangent sections were designated North (N) and South (S) while the curve sections were called East (E) and West (W). The layout of the test track is shown in Figure 4-1.

The primary objective of the test track study was to evaluate the performance of the various surface mixtures. Therefore, each participating agency was able to design 
surface mixtures that were placed as the top $100 \mathrm{~mm}$ (4 in.) of their test sections. Below this the surface, the entire test track had the exact same structure. The pavement was designed for 30 million ESAL to ensure that no structural problems would occur during testing. The application of 10 million ESAL took approximately two years to complete.

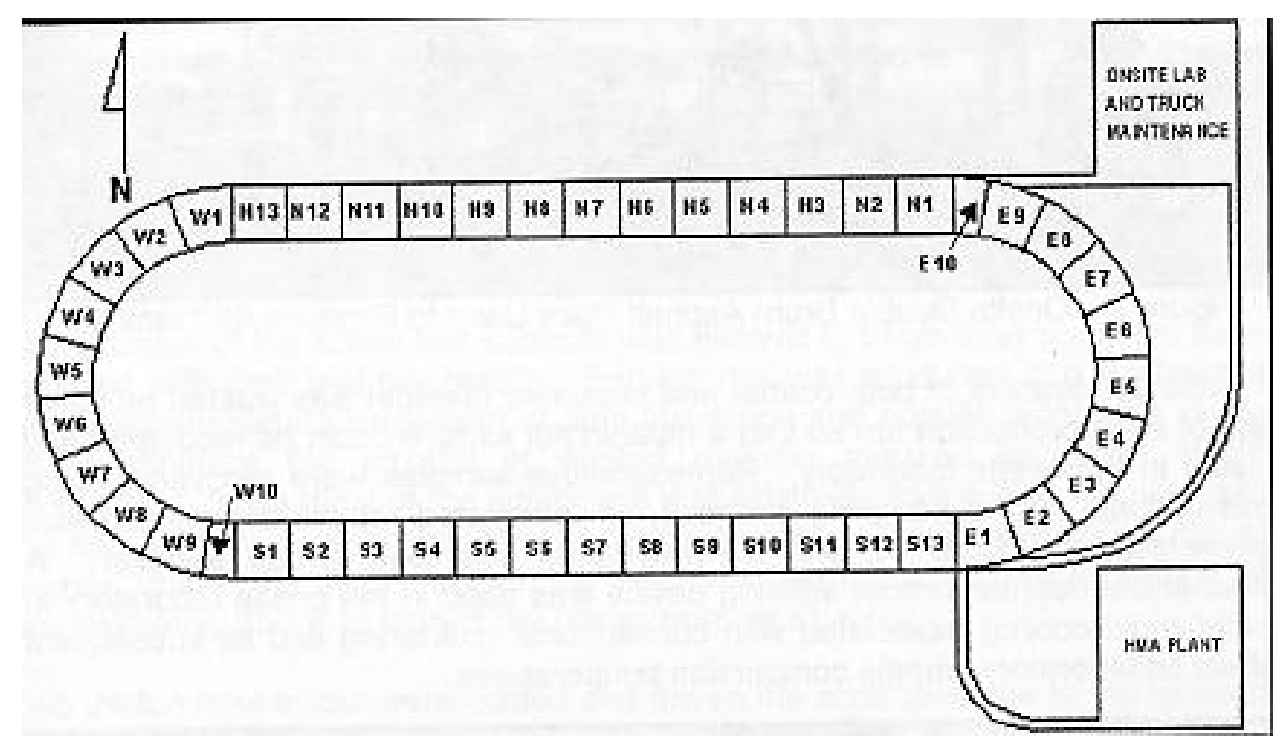

Figure 4-1 Test Track Layout (After Powell, 2001)

\section{$\underline{4.2 \text { Track Construction }}$}

Construction of the track began by clearing the obstructions, including trees, along the intended oval (Brown and Powell, 2001) (Brown et al., 2002). Efforts were made to keep the cut and fill volumes approximately equal. To form a strong foundation for other layers, a 300-mm (12-in.) thick improved subgrade (compacted to 100 percent proctor density) was laid over the finished earthwork. The base course was constructed and compacted on top of the improved subgrade and consisted of 150-mm (6 in.) of crushed aggregate. A 125-mm (5 in.) permeable black base was then placed and it was 
covered by a 375-mm (15 in.) HMA base mixture placed in two lifts. The permeable black base layer was incorporated for drainage purposes. The cross-section of the pavement structure is shown in the Figure 4-2.

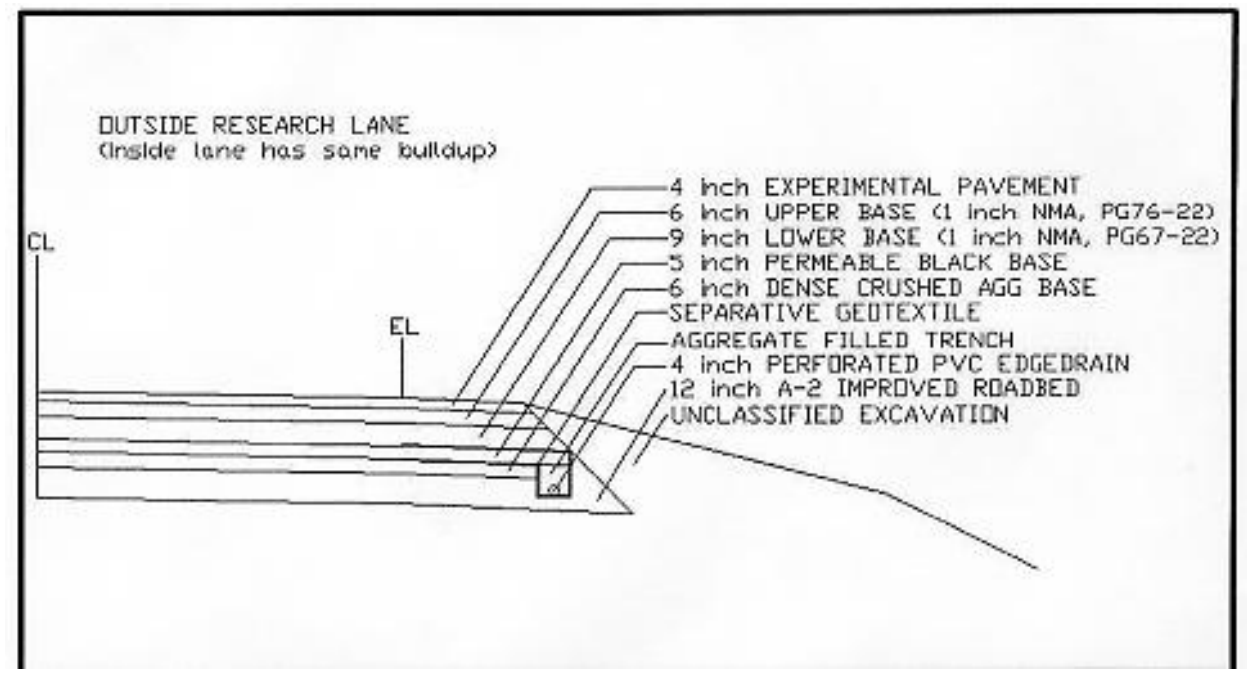

Figure 4-2 Pavement Structure at Test Track (After Powell, 2001)

To minimize hauling, a hot-mix plant was built adjacent to the track. As shown in Figure 4-3, it was a counter flow, double drum plant (Hand et al., 2000) (Powell, 2001). Contact between the burner flames and the binder was thereby prevented as the mixing occurred in the outer drum. Plant operation was programmed to produce $86 \mathrm{Mg}$ (95 tons) of HMA in a single production run. This was enough material to produce one lift in each test section. To provide uniform HMA for the paving operation, the first $18 \mathrm{Mg}$ (20 tons) and the last $14 \mathrm{Mg}$ (15 tons) of HMA produced from a single run was discarded. A 68Mg (75-ton) capacity surge bin was used to store the HMA to be used on the track. Once the first $18 \mathrm{Mg}$ (20 tons) of HMA were produced in a single production run, it was loaded 
into a truck and discarded. The remaining HMA (about $68 \mathrm{Mg}$ (75 tons)) was stored in the surge bin and plant production for the single run was stopped. This procedure eliminated the need for storage silos and preheated the surge bin as well. Two 22-Mg (24ton) trucks were used to haul the HMA from the plant to the paving site.

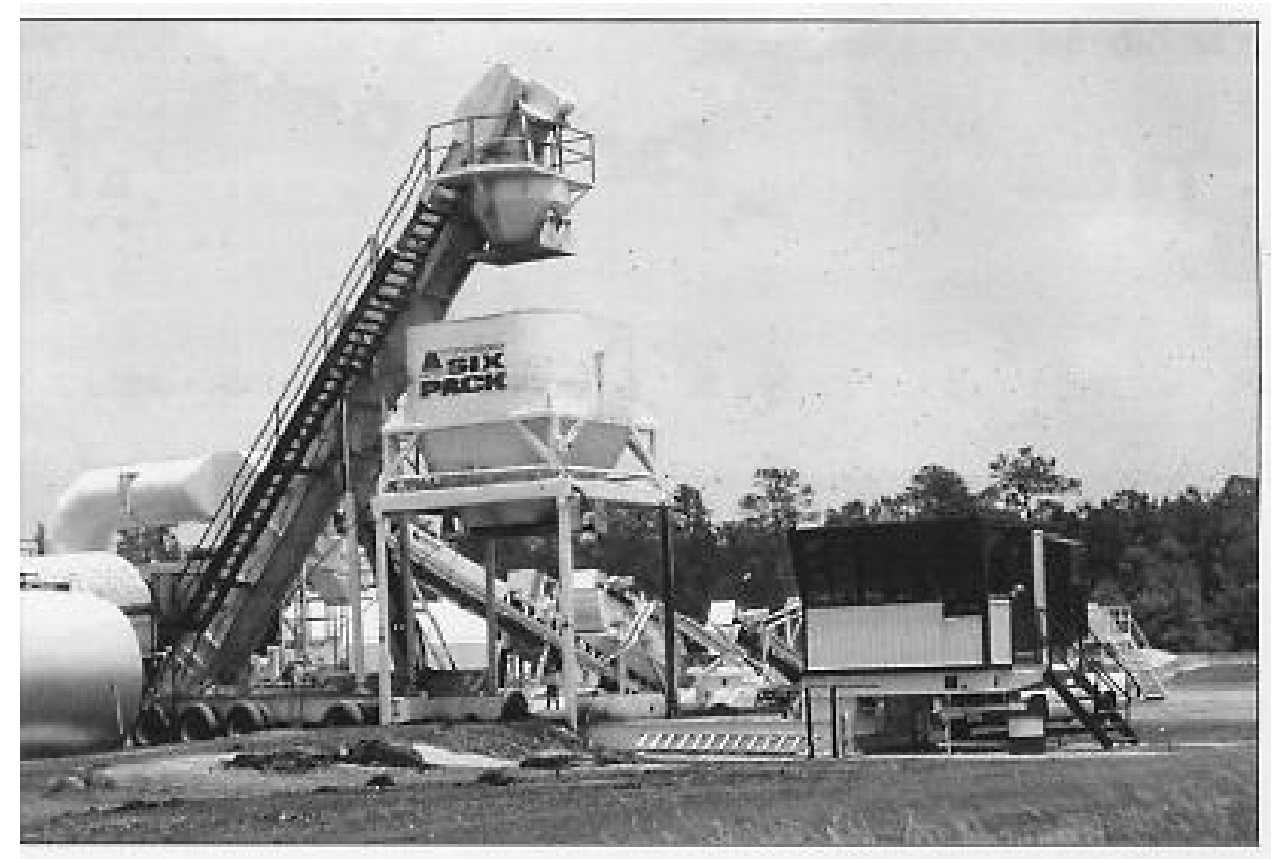

Figure 4-3 Double Barrel Drum Plant (After Hand et al., 2000)

The laboratory mixture design data were used to begin initial plant runs of the HMA mixtures. These were tested for binder content and gradation and the plant settings adjusted as needed until the plant production achieved the target values (Powell, 2001). The hot-mix plant was operated at 182.2 and 160C (360 and 320F) for modified and neat binders respectively (Hand et al., 2000). Since the plant was not operated continuously, it was necessary to apply higher end temperatures during production. 
For each test section the 100-mm (4-in.) thick experimental surface layer was placed in two lifts of 50-mm (2 in.). The inside lane was constructed first so that the results could be used to determine the rolling pattern for the more critical outside lane. Further, the corrections for the nuclear density testing of the outside lanes were obtained using destructive coring of the inside lanes (Brown and Powell, 2001) (Powell, 2001) (Brown et al., 2002). The limited length of the test sections resulted in the placement of the top and bottom lifts on different days.

Every effort was taken to prevent segregation in the HMA mixtures. Mixtures were loaded into the haul trucks using multiple drops from the surge bin and a Material Transfer Vehicle (MTV) was used to load the HMA from the haul trucks into the paving machine. This reduced mixture and temperature segregation (Hand et al., 2000). The paving operation was performed continuously in the direction of intended traffic. Generally, two conventional, gravity-feed pavers were used for placement operations, one each for the inside and outside lanes (Powell, 2001). The width of the outside lane (research lane) was $3.6 \mathrm{~m}$ (12 ft) with a.1.2 m (4 ft) shoulder attached. The transverse slope of the test lane and shoulder were two percent and four percent, respectively. The outside lane and shoulder were paved in a single placement operation using an extended screed. Figure 4-4 shows the typical paving operation.

An $11 \mathrm{Mg}$ (11.8 tons), 2 m (78 in.) wide vibratory double drum roller was utilized to compact the mat. The roller was initially used in the vibratory mode and subsequently in the static mode for the breakdown stage and in the static mode for the finish rolling. Rolling was performed at low amplitude and high frequency for the fine-graded mixtures while high amplitude and high frequency were utilized for the coarse-graded mixtures 
(Hand et al., 2000). At the completion of each pass, the density and temperature of the mat were monitored using nuclear density gauges and infrared thermometers, respectively.

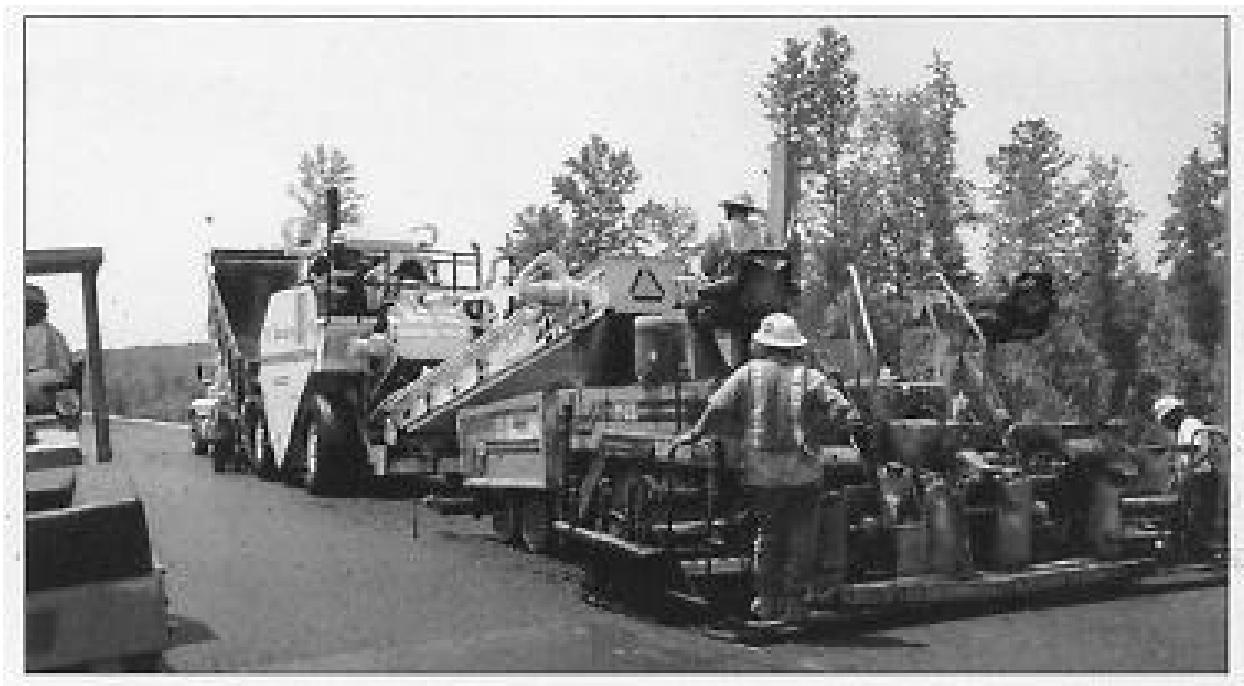

Figure 4-4 Typical Paving Operation (After Hand et al., 2000)

\subsubsection{Quality Control Tests}

Representative samples were collected at different stages of the track construction for quality control testing. Sampling locations included the haul truck, the extruded mat, cold feed belt, and cores from the finished pavement.

A remote truck-sampling device (RTSD) as shown in Figure 4-5 was utilized to collect samples from the hauling trucks after the HMA was loaded from the surge bin. The RTSD utilizes a hollow sampling tube and consists of a sampler with a clamshell (Hand et al., 2000). The clamshell was driven into the HMA to an approximate depth of $0.3 \mathrm{~m}$ (12 in.) and was then opened. After advancing an additional $0.6 \mathrm{~m}$ (24 in.) into the HMA, the clamshell was closed. The sample was then transferred into 5-gallon buckets. 
Asphalt binder contents were determined from the samples using the following test methods:

1. Reflux (Solvent extraction) (AASHTO T 164);

2. Nuclear (American Society for Testing and Materials (ASTM) D 4125); and

3. Ignition test (AASHTO TP53).

The mixture aggregate gradation was also determined from the extracted aggregate. Mixture volumetrics (AASHTO TP4) were determined as well.

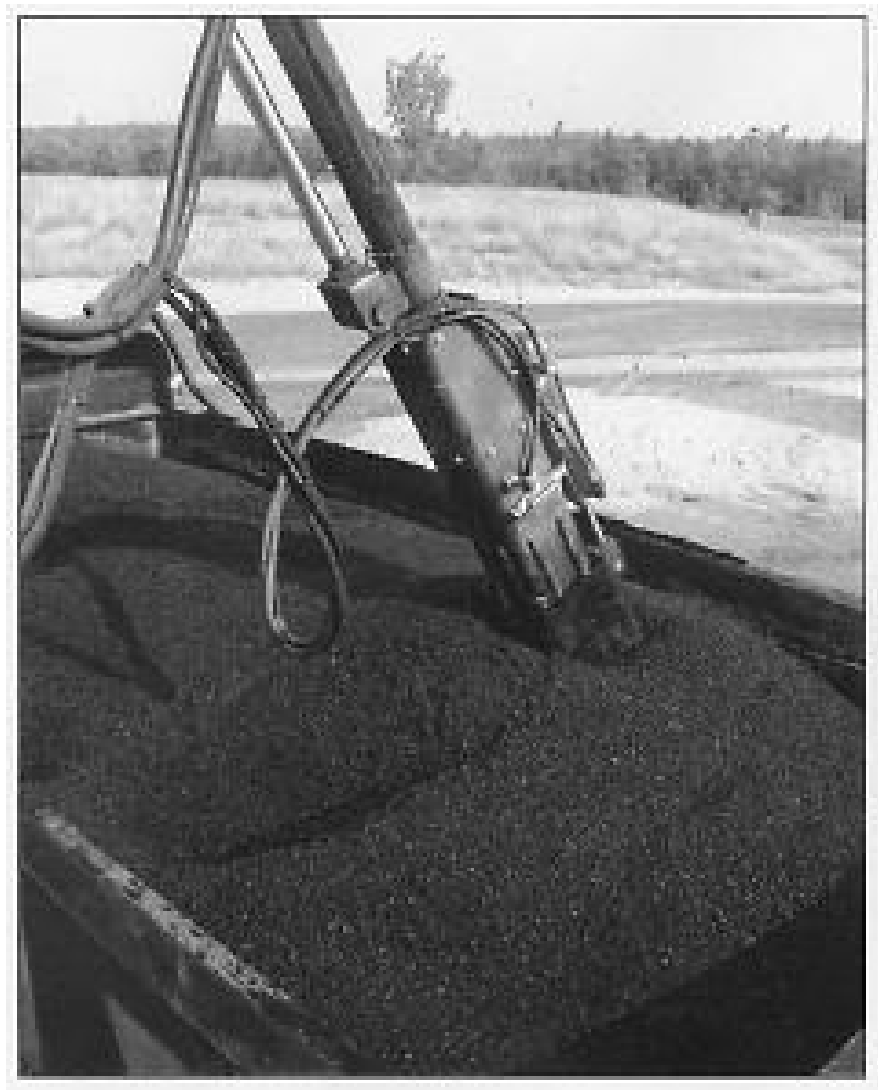

Figure 4-5 RTSD Sampling (After Hand et al., 2000) 
A belt sampler was employed to collect aggregate samples while the plant was in operation using a sweeping technique. These samples were used to obtain the aggregate gradations of the mixture being produced according to AASHTO T11 and T27.

In-place density (ASTM D2726) was measured on cores taken from the inside lane and a nuclear density gauge was used to measure the in-place density of the fresh mat on the outside lane (ASTM D2950). The mat temperature was determined using an infrared thermometer. The as-constructed test track mixture properties are shown in Table 4-1.

Table 4-1 As-Constructed NCAT Test Track Mixture Properties (Powell, 2001)

\begin{tabular}{|c|c|c|c|c|c|c|c|c|c|c|}
\hline Test Section & N1 & N2 & N3 & N4 & N5 & N6 & N7 & N8 & N9 & N10 \\
\hline Gradation ${ }^{1}$ & ARZ & ARZ & ARZ & ARZ & BRZ & BRZ & BRZ & BRZ & BRZ & BRZ \\
\hline Aggregate & $\begin{array}{c}\text { Lms/ } \\
\text { Slag }\end{array}$ & $\begin{array}{c}\text { Lms/ } \\
\text { Slag }\end{array}$ & $\begin{array}{c}\text { Lms/ } \\
\text { Slag }\end{array}$ & $\begin{array}{c}\text { Lms/ } \\
\text { Slag }\end{array}$ & $\begin{array}{c}\text { Lms/ } \\
\text { Slag }\end{array}$ & $\begin{array}{c}\text { Lms/ } \\
\text { Slag }\end{array}$ & $\begin{array}{c}\text { Lms/ } \\
\text { Slag }\end{array}$ & $\begin{array}{c}\text { Lms/ } \\
\text { Slag }\end{array}$ & $\begin{array}{c}\text { Lms/ } \\
\text { Slag }\end{array}$ & $\begin{array}{c}\text { Lms/ } \\
\text { Slag }\end{array}$ \\
\hline Sieve Size & \multicolumn{8}{|c|}{ Percent Passing } \\
\hline 1” & 100 & 100 & 100 & 100 & 100 & 100 & 100 & 100 & 100 & 100 \\
\hline 3/4” & 100 & 100 & 100 & 100 & 100 & 100 & 100 & 100 & 100 & 100 \\
\hline 1/2” & 100 & 99 & 99 & 99 & 99 & 99 & 98 & 99 & 99 & 98 \\
\hline 3/8” & 92 & 90 & 91 & 91 & 84 & 85 & 83 & 85 & 87 & 84 \\
\hline No. 4 & 69 & 66 & 68 & 68 & 52 & 54 & 52 & 55 & 57 & 51 \\
\hline No. 8 & 52 & 50 & 51 & 52 & 38 & 37 & 36 & 37 & 40 & 34 \\
\hline No. 16 & 33 & 33 & 33 & 35 & 26 & 25 & 24 & 24 & 26 & 23 \\
\hline No.30 & 22 & 22 & 22 & 23 & 18 & 17 & 17 & 17 & 19 & 17 \\
\hline No.50 & 15 & 16 & 15 & 15 & 14 & 13 & 13 & 13 & 14 & 13 \\
\hline No. 100 & 10 & 11 & 10 & 9 & 11 & 10 & 10 & 10 & 11 & 10 \\
\hline No. 200 & 6.7 & 7.6 & 6.5 & 6.0 & 8.3 & 8.2 & 7.8 & 7.5 & 8.8 & 7.7 \\
\hline Air Voids, \% & 4.9 & 5.3 & 5.9 & 6.6 & 6.2 & 5.6 & 6.1 & 5.3 & 5.5 & 5.3 \\
\hline Pb, $\%$ & 7.4 & 7.8 & 7.6 & 6.8 & 6.9 & 6.8 & 6.9 & 6.6 & 6.7 & 6.8 \\
\hline Modifier & SBS & SBS & NA & NA & NA & NA & SBR & SBR & SBS & SBS \\
\hline
\end{tabular}

\subsubsection{Instrumentation}

As the pavement structure was similar for all test sections, a decision was made to install only temperature and moisture gauges (Brown et al., 2002). The configuration of 
the temperature probes in the pavement structure is shown in Figure 4-6. They were installed at four depths:

1. Top of the surface layer;

2. Mid-depth of the surface layer (0.05 m (2 in.) below the surface);

3. Bottom of the surface layer ( $0.1 \mathrm{~m}$ (4 in.) below the surface); and

4. Bottom of the upper binder course ( $0.25 \mathrm{~m}$ (10 in.) below the surface).

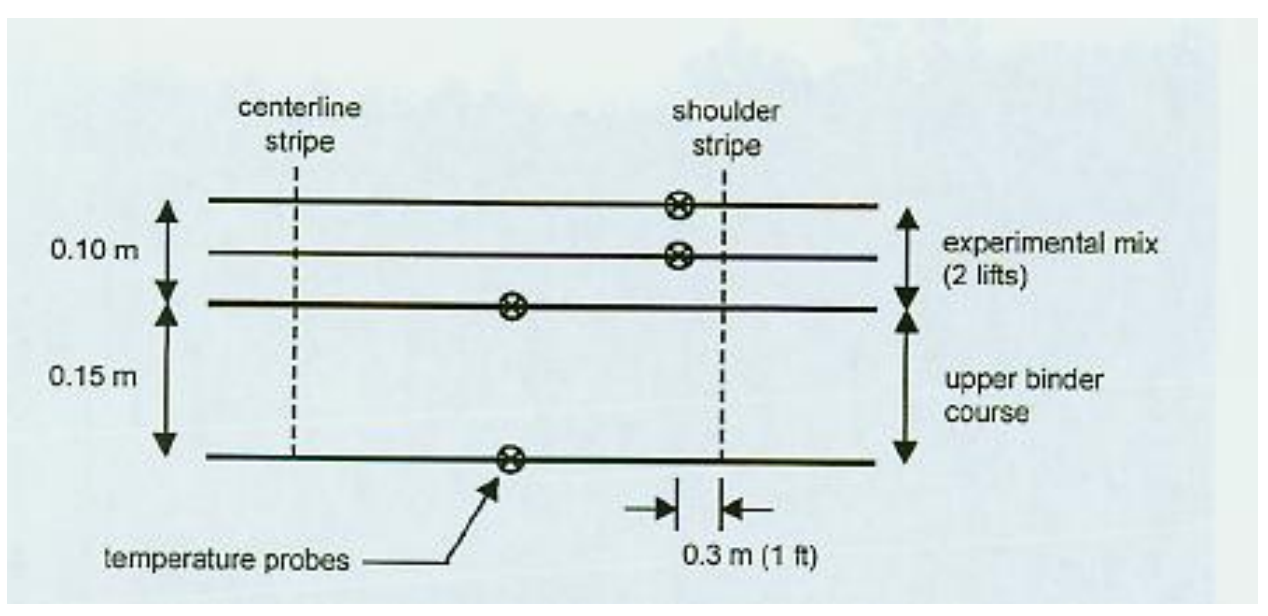

Figure 4-6 Temperature Probe Locations (After Brown et al., 2002)

The probes installed at the depths indicated in 3 and 4 above were located in the center of the outer lane. The probes described in items 1 and 2 above were located $0.3 \mathrm{~m}(1 \mathrm{ft})$ from the outside edge of the outside lane. The data from the temperature probes were recorded to data loggers and transferred to the data acquisition computers every hour. Each data logger served two test sections and software was used for the automated data collection. For every hour, the average temperature, maximum temperature, and minimum temperature were processed for each section. 
Since the test sections were built over both cut and fill, moisture gauges were placed to monitor moisture changes in the subgrade. The moisture gauges were installed at a depth of $0.075 \mathrm{~m}$ (3 in.) below the surface of the subgrade, directly below the alternate transverse joints.

\subsubsection{Traffic Application}

Four tractor-trailer assemblies applied traffic to the track. Each tractor $(13,636 \mathrm{~kg}$ (30,000 lbs) towed three trailers, each loaded to 18,182 kg (40,000 lbs) with 9,091 kg (20,000 lbs) each per axle. This resulted in a total truck weight of 68,182 kg (150,000 lbs) (Brown, 1998) (Brown and Powell, 2001) (Powell, 2001) (Brown et al., 2002). The truck configuration is shown in Figure 4-7. This configuration results in the application of approximately 10 ESAL per pass.

The tractor-trailer assemblies were driven at $72 \mathrm{kph}$ (45 mph) around the oval track for 18 hours a day, six days a week, for approximately two years. Trafficking was stopped on each Monday for data collection and maintenance of the truck assemblies. A vehicle identification system was installed at the track to record the ESAL applications (Brown et al., 2002). Further, as a backup measure, driver logs and counters were used to maintain accurate ESAL application records. 


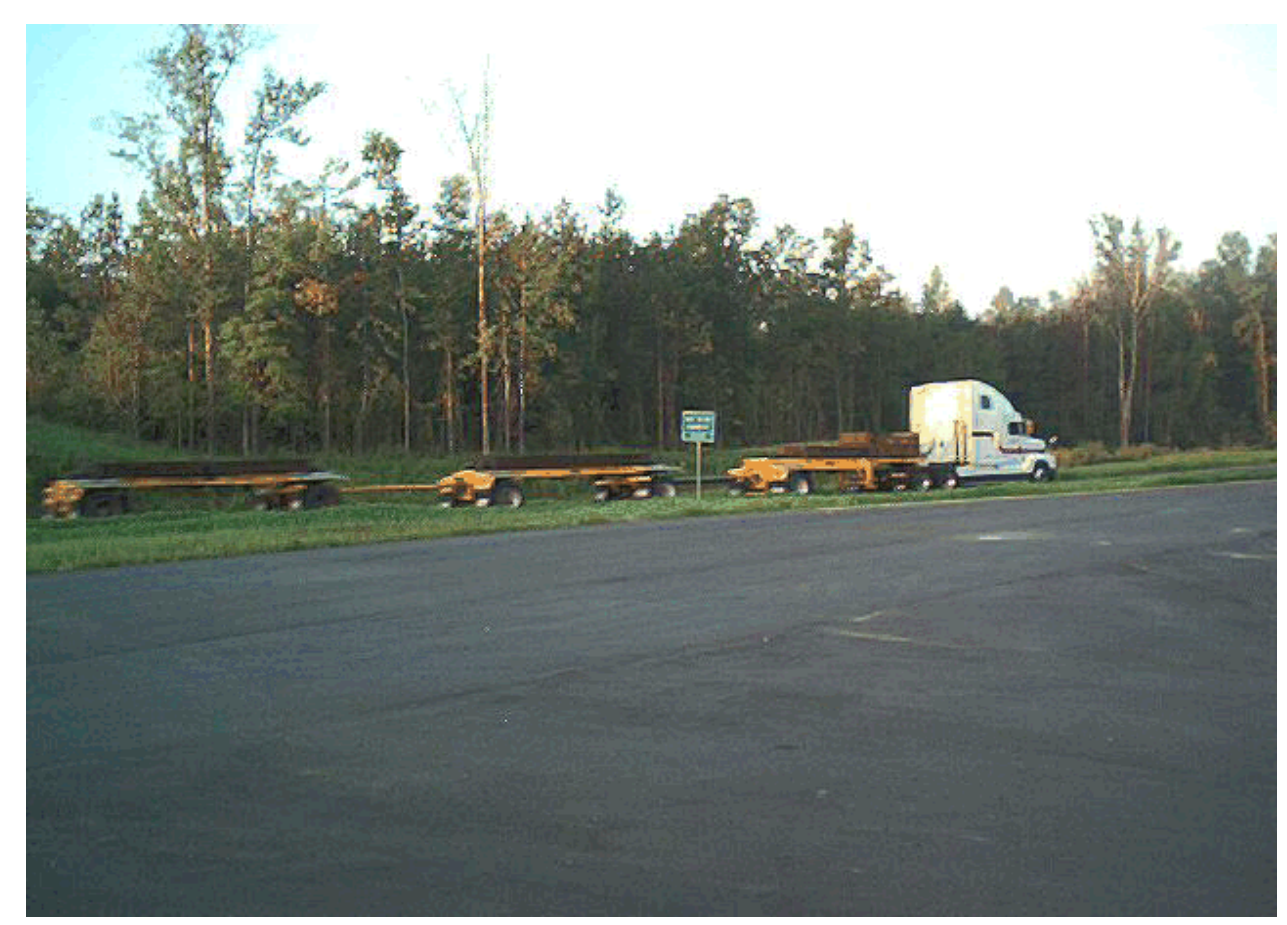

Figure 4-7 Truck Configuration (NCAT Website)

\subsubsection{Performance Data Collection}

Rutting data were obtained using laser technology and transverse profilers (Brown et al., 2002) (Powell, 2002). To eliminate biased readings, random number principles were applied to establish the location of the transverse profiles for the rutting measurements. As shown in Figure 4-8, a dipstick was used to determine the transverse profile at each location. Each $60 \mathrm{~m}$ (200 ft) long test section had the center $45 \mathrm{~m}(150 \mathrm{ft})$ of the section divided into three sub-lots (replications). This resulted in three replicates of rutting data available for each section. The $7.5 \mathrm{~m}$ (25 ft) long areas on both ends of a section were treated as transition zones. 


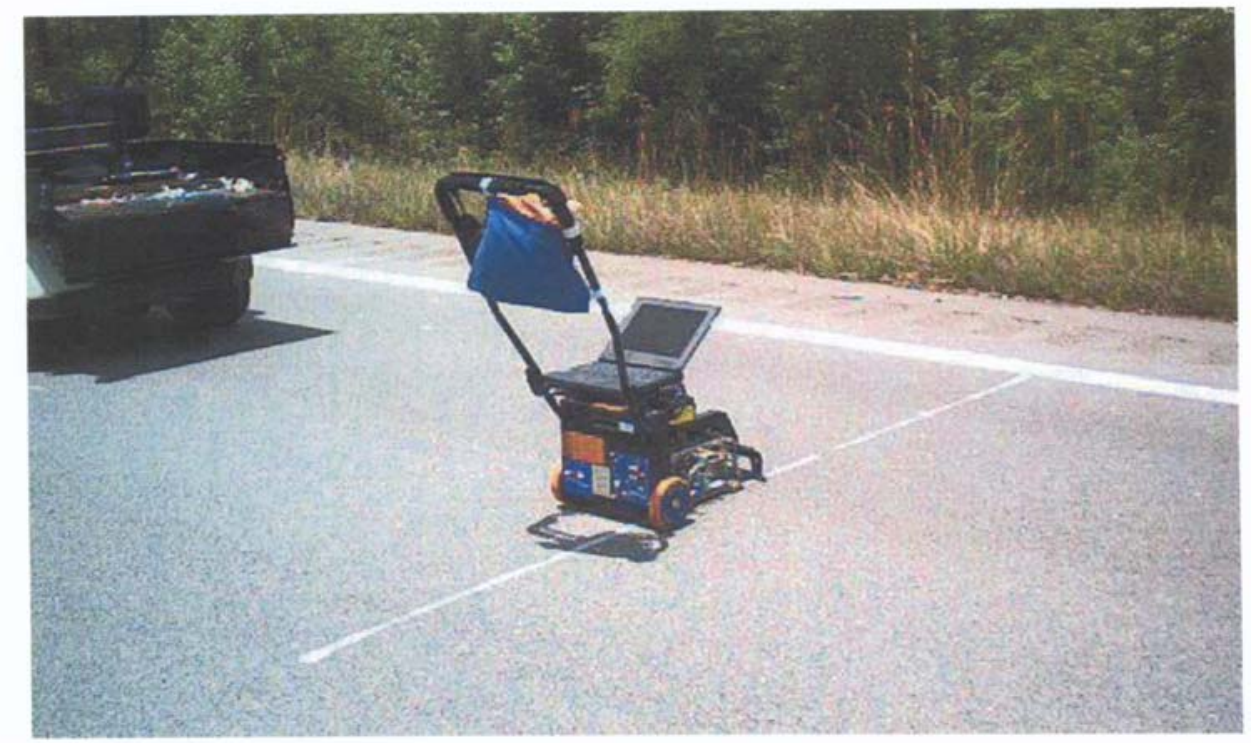

Figure 4-8 Transverse Profile Data Collection with Dipstick (After Powell, 2002)

Two deflection tests per section were also performed on a monthly basis, as well as surface friction (wet skid) tests. Cores were extracted every three months for testing, and wheel path densification was measured using nondestructive testing techniques such as nuclear gauge and non-nuclear devices. 


\section{CHAPTER 5 TEST METHODS}

\subsection{Accelerated Pavement Tester}

\subsubsection{Introduction}

In 1992, a prototype full-scale accelerated loading facility was designed and built under a cooperative agreement between INDOT and Purdue University. Named the Accelerated Pavement Tester, or APT for short (Galal and White, 1999) (Stiady, 2000) (Hua, 2000) (Fang, 2000), its primary purpose is to accelerate pavement damage on the test sections constructed at the facility. This can be done by a combination of high temperature, increased load, and slow load application.

Being able to control the test temperature, loading conditions, and materials allows the effects of these variables on rutting performance to be investigated using the APT. Further, other factors, such as pavement structure and construction techniques, can also be evaluated. For example, new materials, and mixture design methods can be evaluated for optimal rutting performance before they are used in actual pavements.

\subsubsection{Geometry}

The APT consists of a loading carriage that travels over pavement sections that are built in the test pit. The test pit is $6 \mathrm{~m}$ (20 ft) square and has a depth of approximately $2 \mathrm{~m}(6 \mathrm{ft})$. The cross section of the pavement structure used in this study is shown in 
Figure 5-1. It consisted of $1.4 \mathrm{~m}$ (4.6 ft) of compacted pea fill over which $0.3 \mathrm{~m}$ (12 in.) thick heavily reinforced concrete slabs were placed. Each slab was $1.5 \mathrm{~m}$ (5 ft) wide. Thus four were used to completely cover the area as shown in Figure 5-2. The HMA test mixtures were placed over the concrete slabs and were $0.1 \mathrm{~m}$ (4 in.) thick.

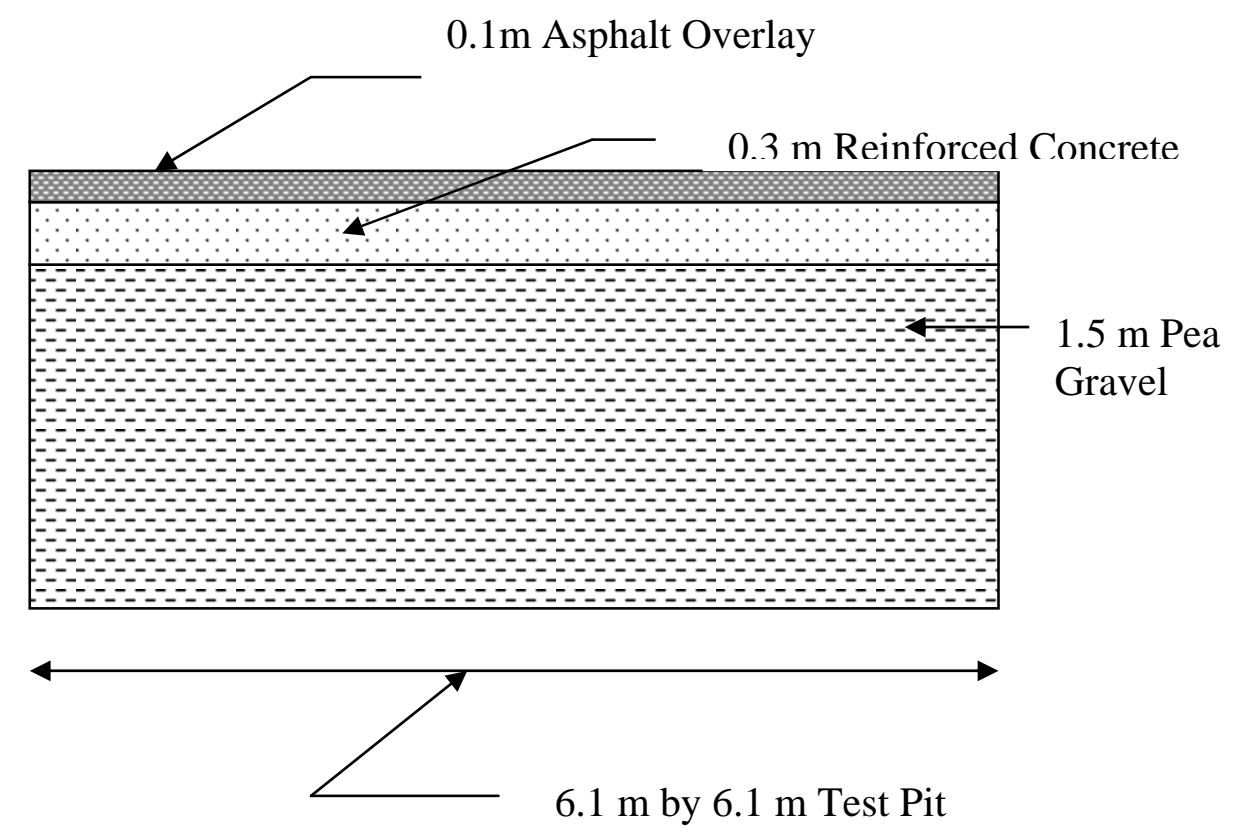

Figure 5- 1 Typical APT Pavement Cross Section 


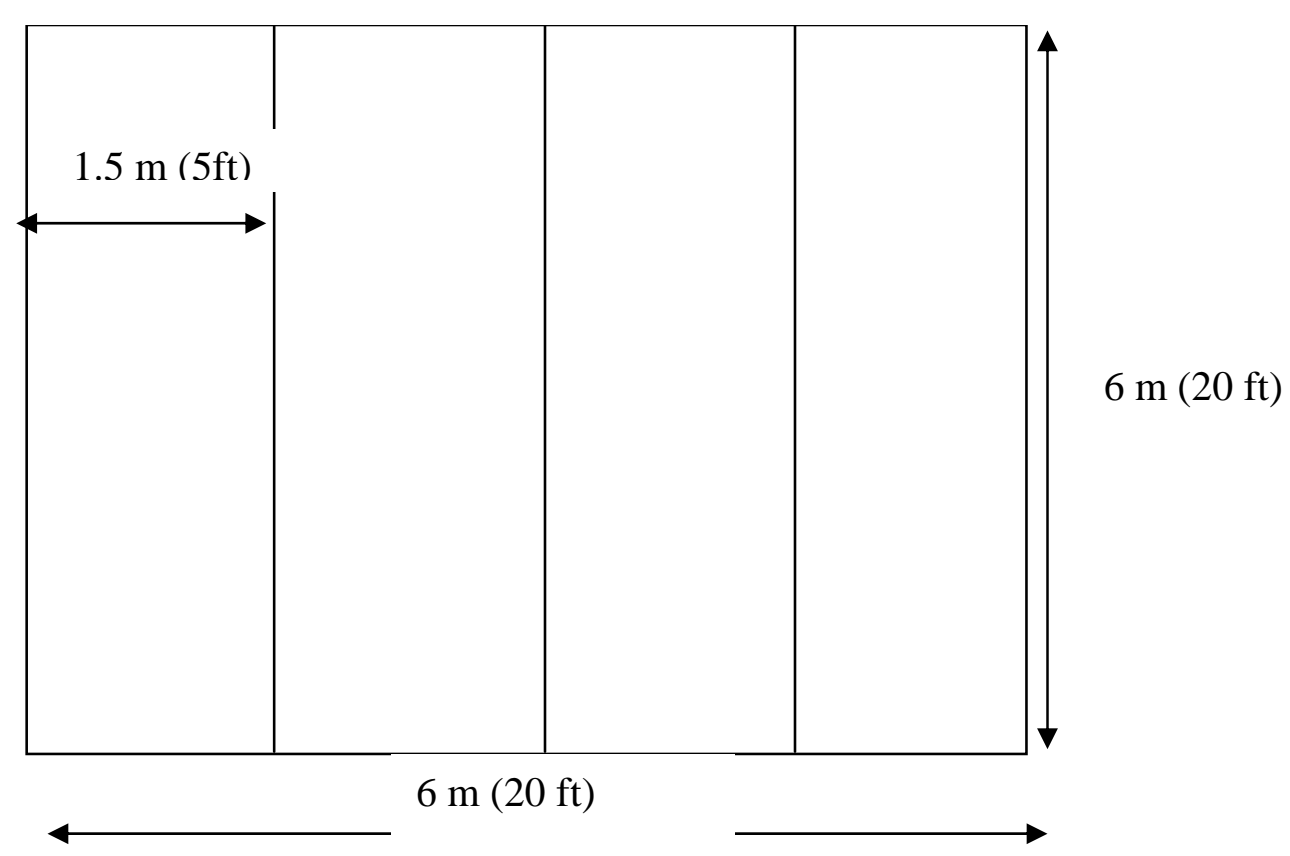

Figure 5-2 APT Test Section Layout

\subsubsection{Operational Details}

The APT pavement temperature is controlled by two heating systems (Huang, 1995) (Galal and White, 1999) (Stiady, 2000) (Hua, 2000) (Fang, 2000). The test sections are heated from below using hot water flowing through hoses embedded in the concrete slabs as shown in Figure 5-3. Each slab can be heated independently. A radiant heating system in the ceiling is used to minimize the pavement/air temperature difference therefore allowing a uniform temperature to be maintained in the facility, even during the winter months. Typical pavement test temperatures range from 20 to 60C (68 to 140F). APT traffic can be applied with or without wander in one or two directions. The testing carriage is capable of applying a load up to $89 \mathrm{kN}$ (20,000 lbs) on a single or dual wheel assembly using springs in tension and compression to exert a constant load on the wheels. This type of loading mechanism is unique and provides energy savings over a 
mass loading system. Four pneumatic cylinders are used to help lift the wheels so they can be returned to their original starting position in the case of one-directional traffic. Transverse movement of the wheels for traffic wander is provided by an electric motor and computer program that used a random number generator.

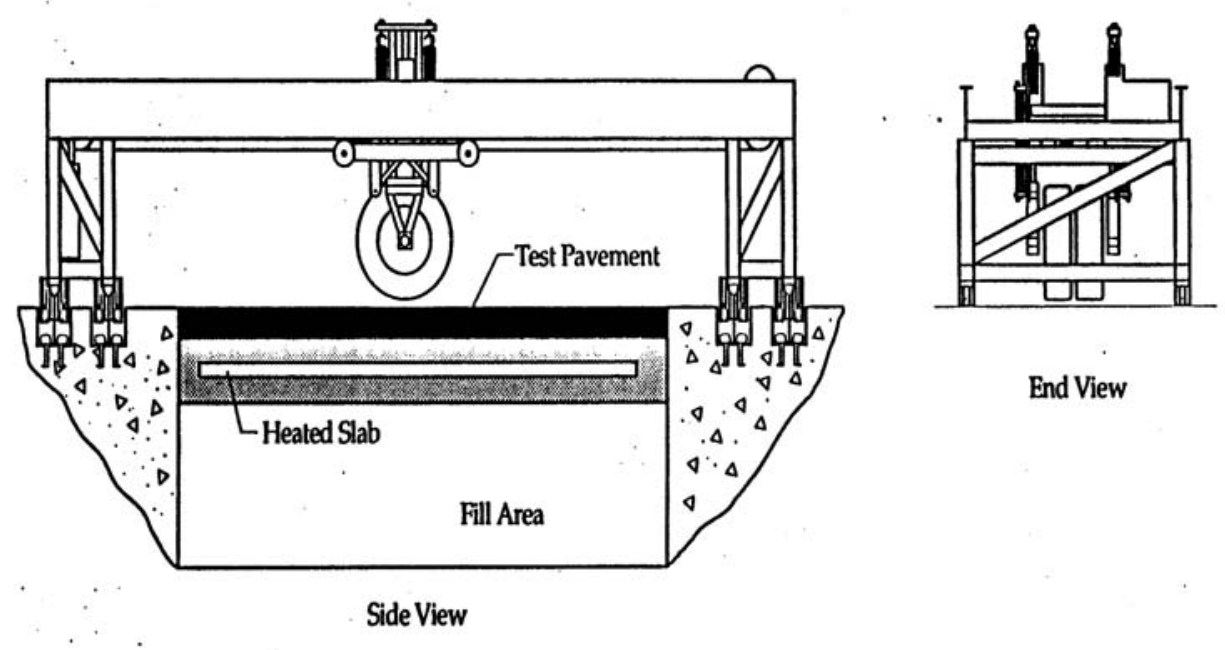

Figure 5-3 Accelerated Pavement Tester Schematic (After Hua, 2000)

For this study, a standard half-axle dual wheel assembly was loaded to $40 \mathrm{kN}$ (9,000 lbs) as shown in Figures 5-3 and 5-4. The wheels were inflated to $690 \mathrm{kPa}(100$ psi). Traffic was applied in one direction with the carriage traveling at $8 \mathrm{kph}(5 \mathrm{mph})$. Wheel wander was not employed in order to further accelerate the rutting damage. 


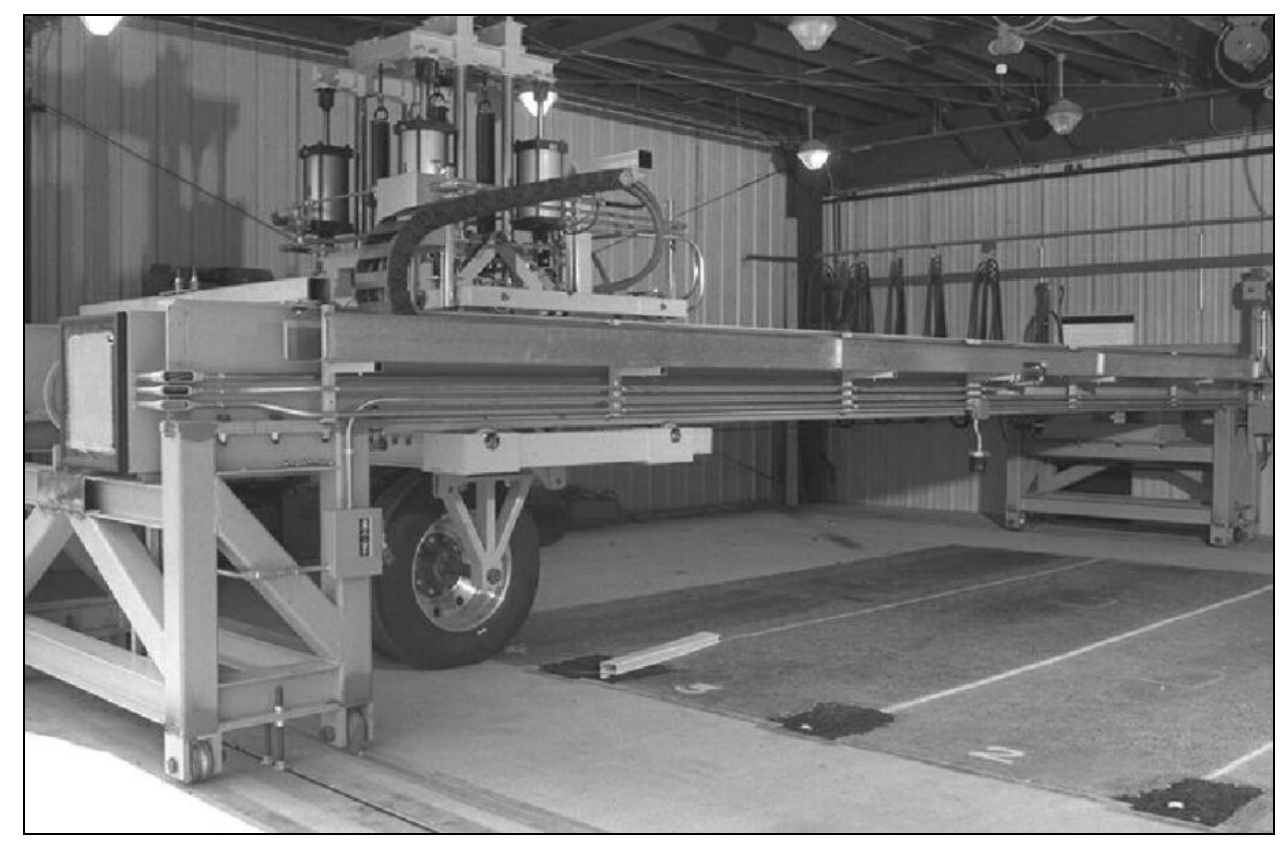

Figure 5-4 APT Loading Configuration (After Hua, 2000)

\subsubsection{Pavement Construction}

The pavement test sections were constructed using conventional HMA

construction methods. Aggregates and binder were transported from Alabama to a local hot-mix plant where the NCAT track mixtures were duplicated (binder content and gradation). Prior to mixture placement, a thin tack coat was applied to facilitate bonding between the concrete slab and the HMA mixtures.

The HMA mixtures were hauled from the hot-mix plant to the APT and loaded into the hopper of the paving machine. The loaded paving machine then backed into the test pit, lowered the screed and placed the mixtures as it traveled out of the facility. Typically, the mixtures were placed in two lifts and the desired density was achieved using static steel-wheeled rollers. During the first construction cycle, the densities were 
monitored using a nuclear density gauge and a similar roller pattern was used in the subsequent construction cycles.

Two test mixtures were placed during each construction cycle. As shown in the Figure 5-2, the pit consists of four test lanes. The paving machine places two identical test lanes in a single pass. The second mixture is then placed on the other half of the test pit. In this study, the same mixture type with two different binder contents (optimum and optimum plus one-half percent) were placed in a single construction cycle.

\subsubsection{Quality Assurance}

Quality procedures followed during test section construction involved obtaining loose mixture samples at the paving machine for laboratory testing. Extraction and gradations were performed on these samples and the results were compared with target gradation and binder content values. Prior to traffic application, cores were taken from the test lanes to determine the initial in-place density of each test section. Additional cores were taken from the test sections after traffic application to determine the in-place density after traffic. Slab specimens were also cut from the test sections prior to traffic application and later used for PURWheel testing.

\subsubsection{Data Collection}

Rutting data from the APT were collected using a profilometer attached to the fixed beams of the APT. Nine transverse cross sections were laid out two feet apart along the longitudinal direction and these locations were used to obtain the transverse profile at predetermined passes as demonstrated in Figure 5-5 (Hua, 2000) (Stiady, 2000). A small 
wheel of the profilometer was lowered and allowed to roll across the transverse direction. Both the vertical and horizontal positions of the profilometer wheel were obtained electronically from the transducers.

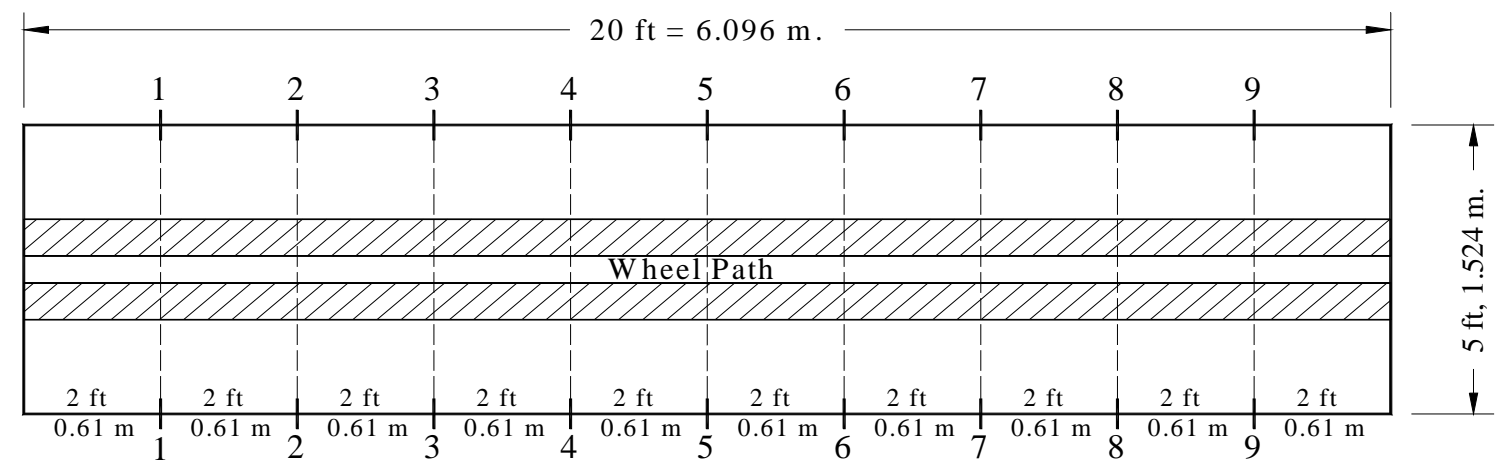

Figure 5-5 APT Profile Data Collection (After Hua, 2000)

Before traffic application an initial transverse profile of each test section was recorded. After application of a predetermined number of passes, another profile was obtained at the same locations. This process was repeated throughout the testing of each section. Computer software automatically recorded the rutting profile data and transferred it to a spreadsheet (Stiady et al., 1998). Rutting measurements were taken after the application of 25, 50, 75, 100, 200, 300, 400, 500, 600, 700, 800, 900, 1,000, 1,500, 2,000, 2,500, 3,000, 4,000, 5,000 wheel passes and at increments of 2,500 passes thereafter until the testing was terminated. Rutting data were obtained more frequently in the initial stages of traffic application due to the higher rut rate that exists in this domain.

Once the profile data were obtained, the measurements were averaged from locations 4 through 6 . The APT carriage needs some distance to accelerate from rest to 
operating speed as well as to decelerate from operating speed to rest. To account for this speed change, the profiles from locations 1 to 3 and 7 to 9 (sections at the ends of the test lanes) were not considered in the rutting calculations. Four types of rutting measurements were made during data collection, total rut depth, rut depth, rise height, and severe rut. As shown in Figure 2-3, rut depth is the deformation measured below the original pavement surface and rise height is the deformation measured above the original surface. Total rut depth is the summation of the absolute value of rut depth and the rise height. Severe rut is simply the maximum rut depth observed at any one of the locations, 4 through 6. Figure 5-6 is a plot of three of the rut depth measurements (severe rut is not included) as a function of APT wheel passes.

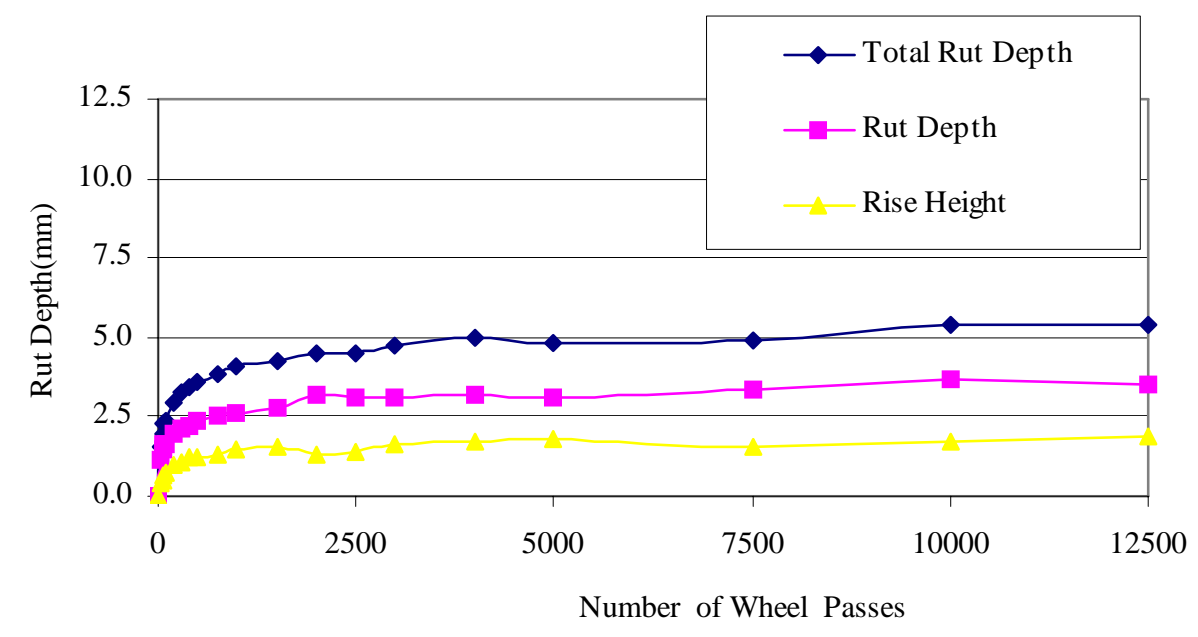

Figure 5-6 Typical APT Rut Data

\subsection{PURWheel Laboratory Tester}

\subsubsection{Introduction}

Laboratory tests that reliably predict in-service rutting performance would be very beneficial to HMA industry. Currently, laboratory loaded wheel testers are commonly 
used for this purpose. They apply moving loads in a condensed amount of time under controlled test conditions. The corresponding rutting data can be conveniently collected and the rutting performance of the HMA mixtures can be compared, ranked, and related to in-service rutting. Many state agencies have begun using such devices to supplement mixture design procedures and to evaluate the rutting potential of HMA mixtures.

The PURWheel testing device located at Purdue University was developed to simulate the conditions associated with rutting and stripping (Huang, 1995) (Pan, 1997) (Stiady, 2000) (Hua, 2000). A photograph of the equipment is shown in Figure 5-7. Capable of establishing and maintaining high temperature and moisture conditions, the PURWheel was the end product of modifications performed on the basic German Hamburg device. Two air cylinders drive two wheels separately and maintain a constant wheel velocity throughout the stroke. A transverse mechanism is used to provide wheel wander and the wheel mounting assembly can accommodate different types of wheels. Additionally, a larger sample box was included to minimize the boundary effects and the testing chamber was modified to facilitate testing in both hot/dry and hot/wet conditions. Rutting is measured over the entire length of a specimen using moving Linear Variable Differential Transducers (LVDT). 


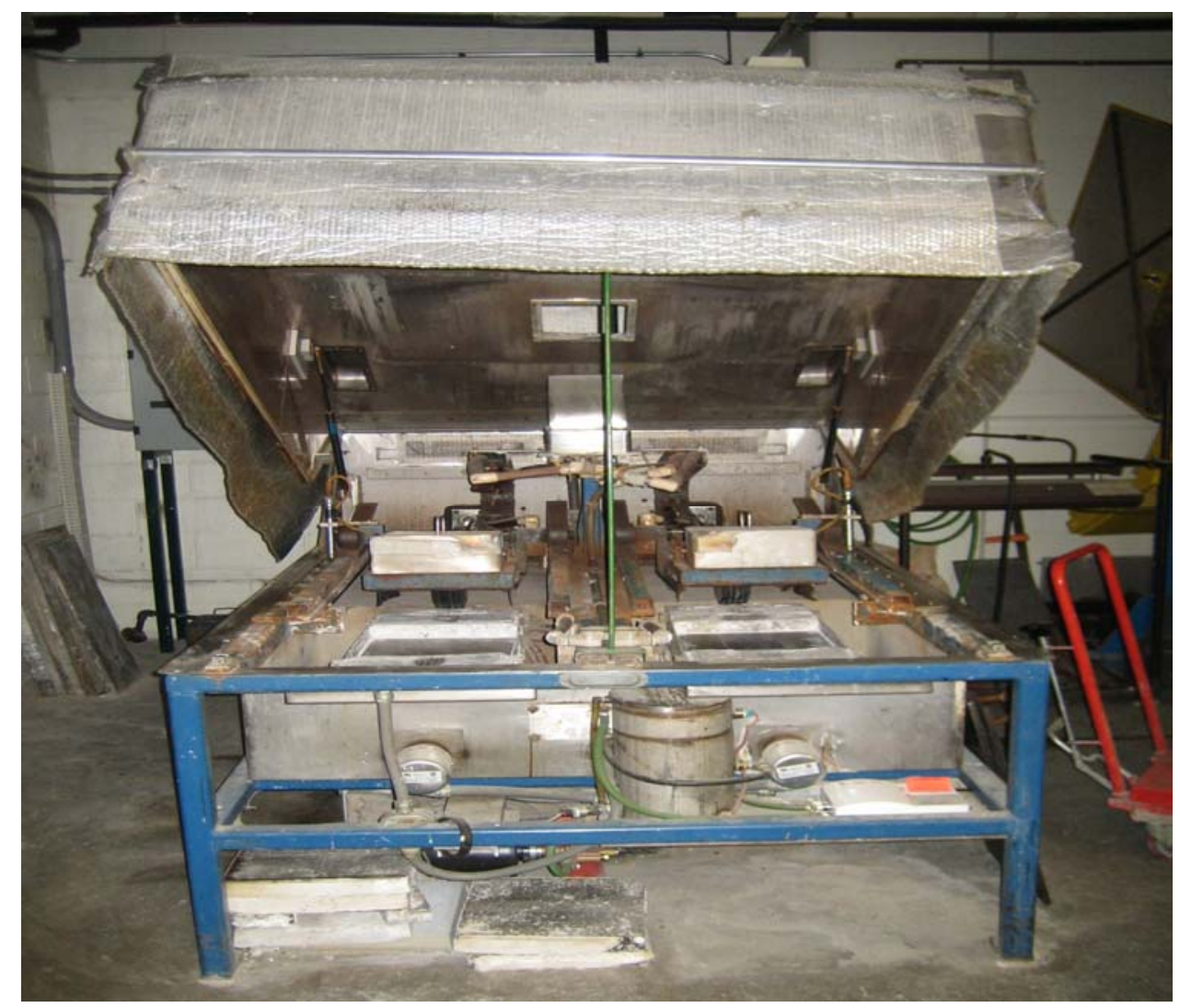

Figure 5-7 PURWheel Testing Device

\subsubsection{Specimen Geometry}

PURWheel specimens are 305 mm (12 in.) long and 290 mm (11.25 in.) wide and are held into the sample boxes using concrete blocks and plaster of Paris. Specimen thickness is determined by the NMAS and is typically $50 \mathrm{~mm}$ (2 in.) for laboratorycompacted specimens. The thickness of the specimens taken from the APT test sections varied from 50 to $100 \mathrm{~mm}$ (2 to 4 in.).

\subsubsection{Linear Compactor}

Laboratory compacted specimens were prepared using the linear compactor. This compactor was developed from the same concept as a similar device used for preparing 
Hamburg test specimens (Habermann, 1994). However, some modifications were made to facilitate the fabrication of larger samples. Test specimens of thickness up to $125 \mathrm{~mm}$ (5 in.) can be compacted in the equipment.

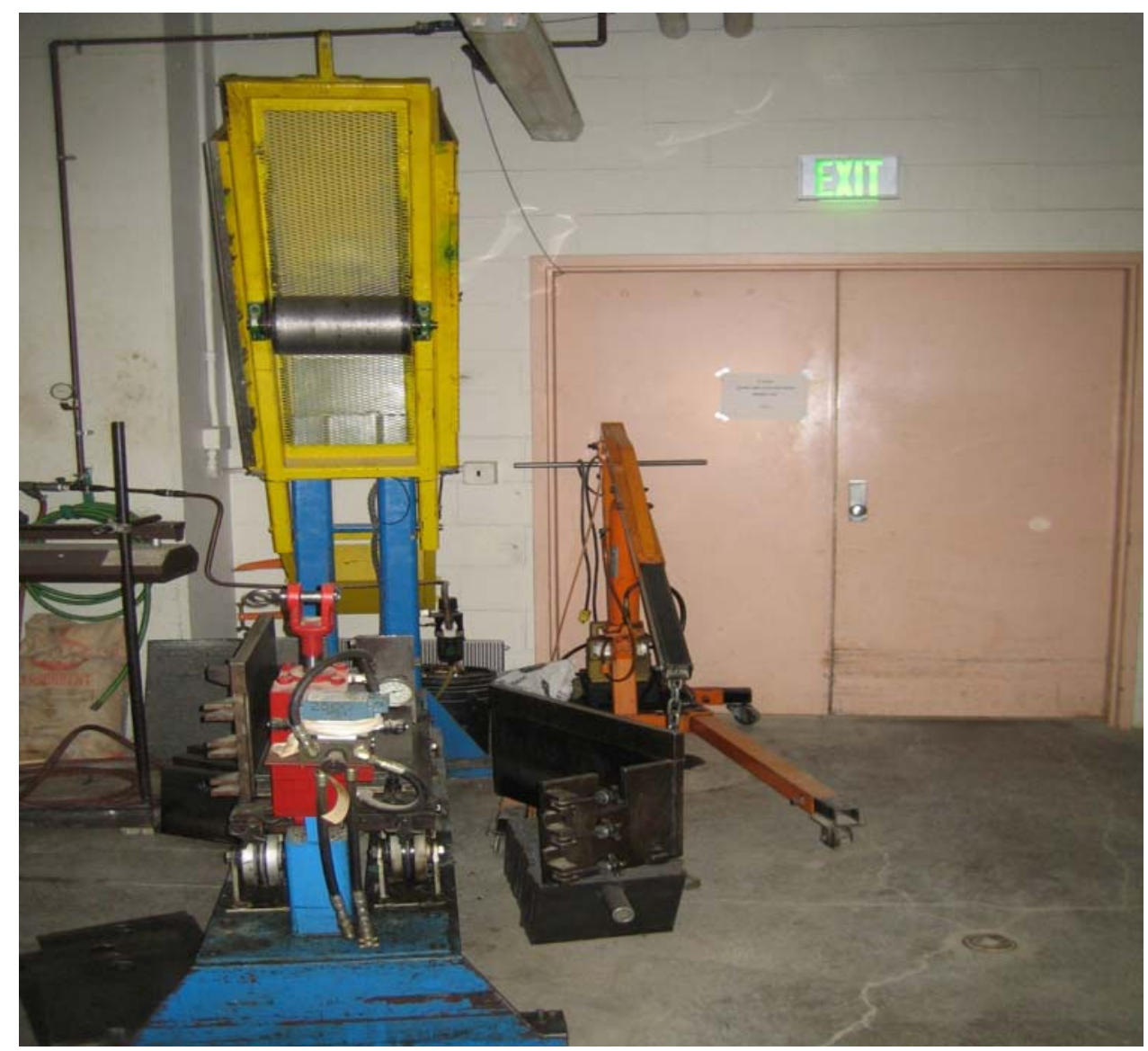

Figure 5-8 Linear Compactor

The main features of the linear compactor include a rectangular steel mold attached to an air cylinder, a loading frame with a steel roller, a set of steel plates, and a hydraulic ram as shown in Figure 5-8. The steel mold is $300 \mathrm{~mm}$ (12 in.) wide by 625 mm (25 in.) long by $368 \mathrm{~mm}$ (14.5 in.) deep and is driven back and forth by an attached air cylinder. The steel plates are 12.5 mm (0.5 in.) thick by 300 mm (12 in.) wide and are 
placed on their edges on top of the HMA mixture and the compactive force is applied by the hydraulic ram through the steel roller onto the steel plates. During the compaction process, each steel plate compacts the HMA mixture independently of the other plates. This action is analogous to compaction by a static steel wheel roller.

\subsubsection{Sample Preparation}

Generally, LMLC specimens are compacted to achieve a specified target density. In this study the target values were equal to the in-place densities found at the NCAT test track for each given mixture. The sample thickness was based on the NMAS of the mixture; in this study the samples were compacted to a thickness of $50 \mathrm{~mm}$ (2 in.). The total mass of material required for compaction was estimated based on the target density and the known compaction box volume. Allowances were made for material loss during the material handling process.

FMFC specimens can also be tested in the PURWheel. In this study specimens were cut from the track and APT test sections prior to traffic application. They were tested in the PURWheel after being trimmed to fit into the sample box.

\subsubsection{Test Parameters}

Test temperature in the PURWheel can vary from room temperature to 65C (145F). The specimens in this study were tested under dry conditions at 50C (122F). There are two heating systems used to provide hot/dry conditions. A heater and pump are used to circulate hot water through conduits located under the specimen-mounting box while an air-heating device is used to minimize the sample/air temperature differential 
using electrical resistance to generate heat as shown in Figure 5-9. Thermocouples installed inside dummy samples provide the necessary feedback data for temperature control.

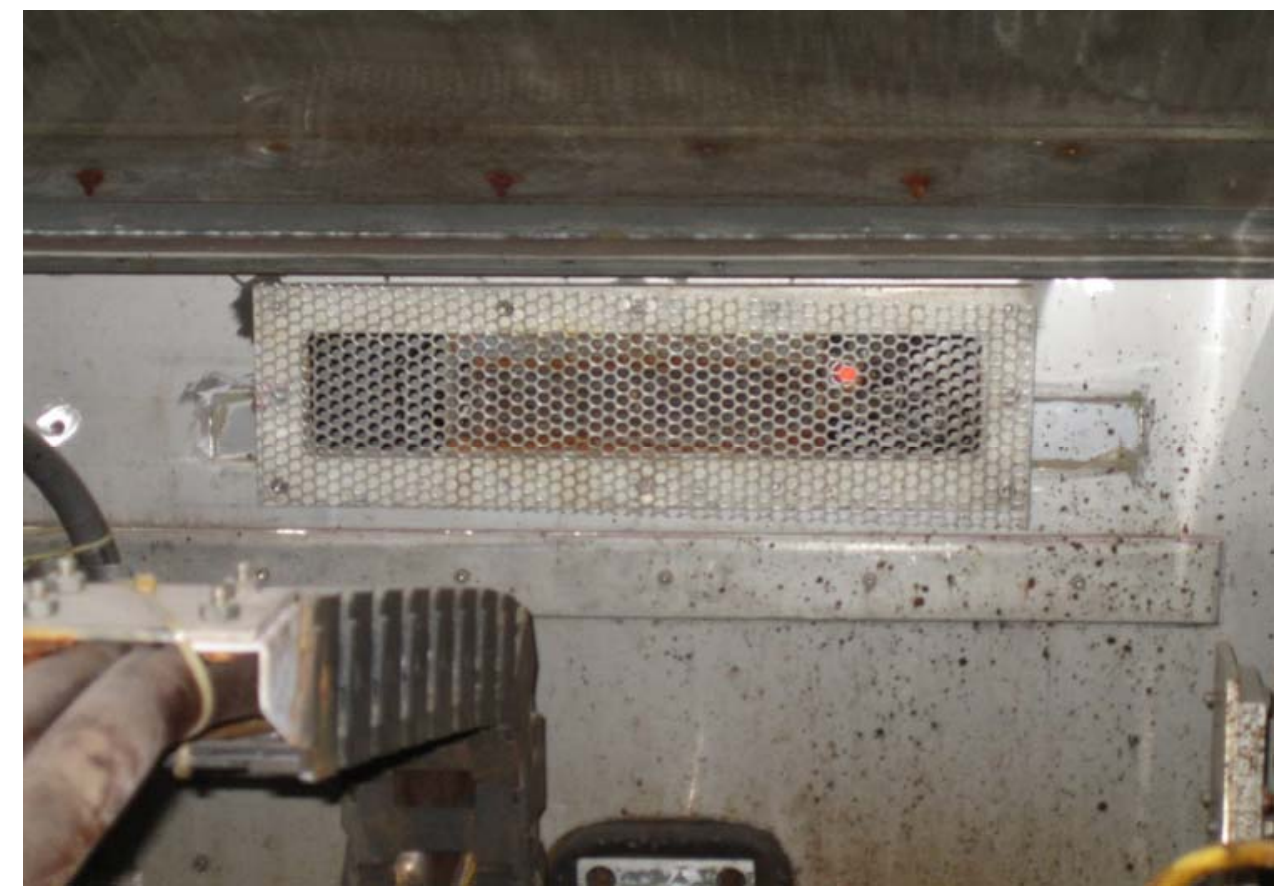

Figure 5-9 PURWheel Air Heater

Two specimens are tested at a time with a wheel velocity of $33 \pm 2 \mathrm{~cm} / \mathrm{s}(13 \pm 0.8$ in./s). The pneumatic tires were inflated to $690 \mathrm{kPa}$ (100 psi) and each wheel was loaded to $1.5 \mathrm{kN}$ (334 lbs). The specimens were tested until 20,000 passes were applied or a 20 mm (0.8 in.) rut was observed, whichever came first. One wheel pass consists of a forward or backward movement of the wheel over the specimen. 


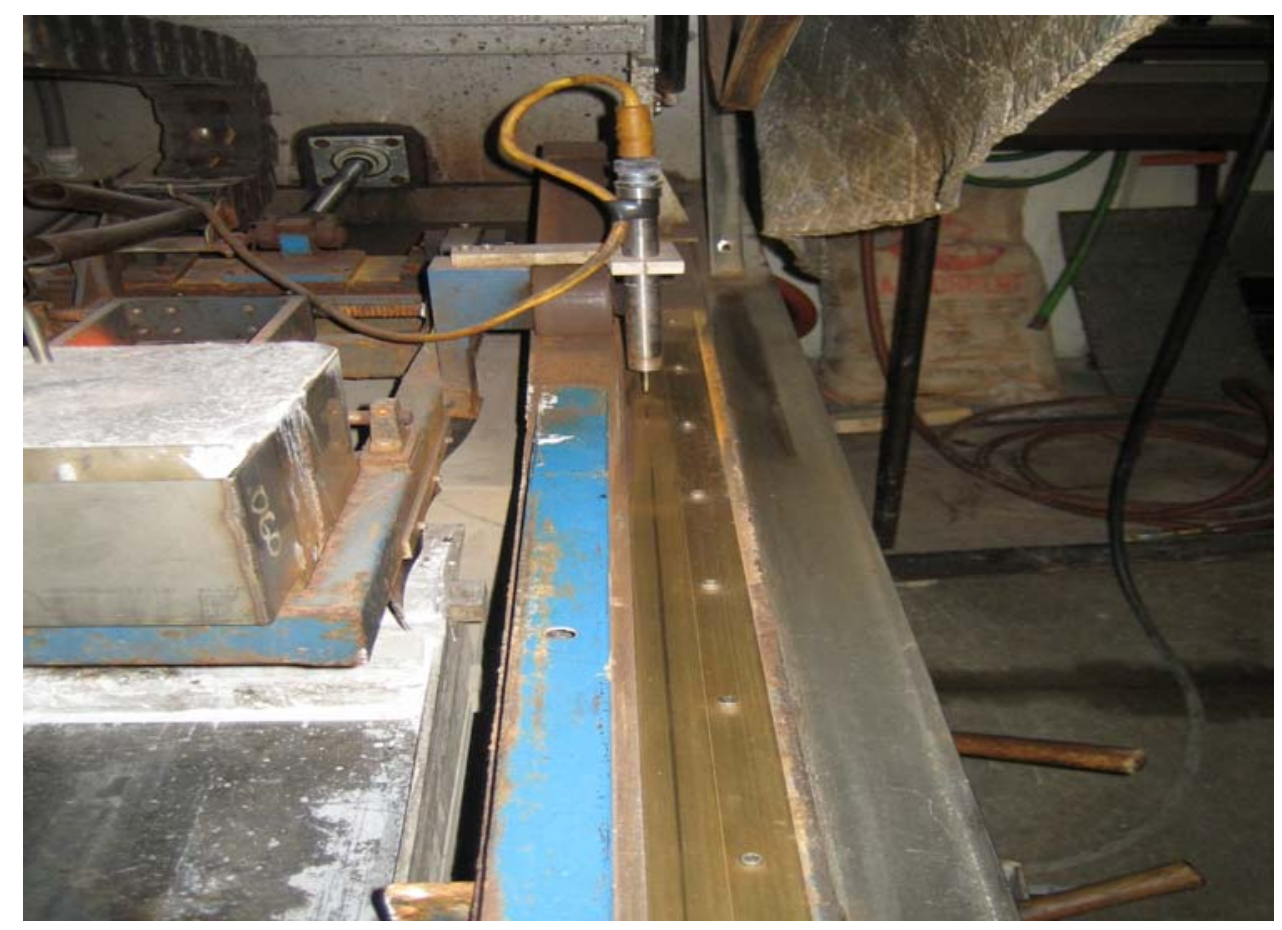

Figure 5-10 PURWheel Linear Transducer (After Hua, 2000)

\subsubsection{Rutting Measurements}

The PURWheel uses transducers to measure rutting. They are attached to the moving parts as indicated in Figure 5-10 so that vertical deformations can be taken at any point on the wheel path over the entire slab length. Rutting data can automatically be collected and recorded at predetermined intervals. For example, in this study, data were recorded every 250 passes. The vertical deformation is measured with respect to the original specimen surface downward; rise height data are not measured using the transducers. This is a drawback of using transducers to measure rutting. At the conclusion of the test, the rise height must be measured manually using a calipers and a T-shaped aluminum bar. A typical plot showing the rut depth history (variation of rut depth with number of wheel passes) of a specimen is presented in Figure 5-11. The transducers are 
subject to electrical noise and the accuracy of the measurement depends on the magnitude of the noise. Stiady (2000) reported an accuracy of $\pm 0.03 \mathrm{~mm}(0.001 \mathrm{in}$.) for rut depth measurements in the PURWheel.

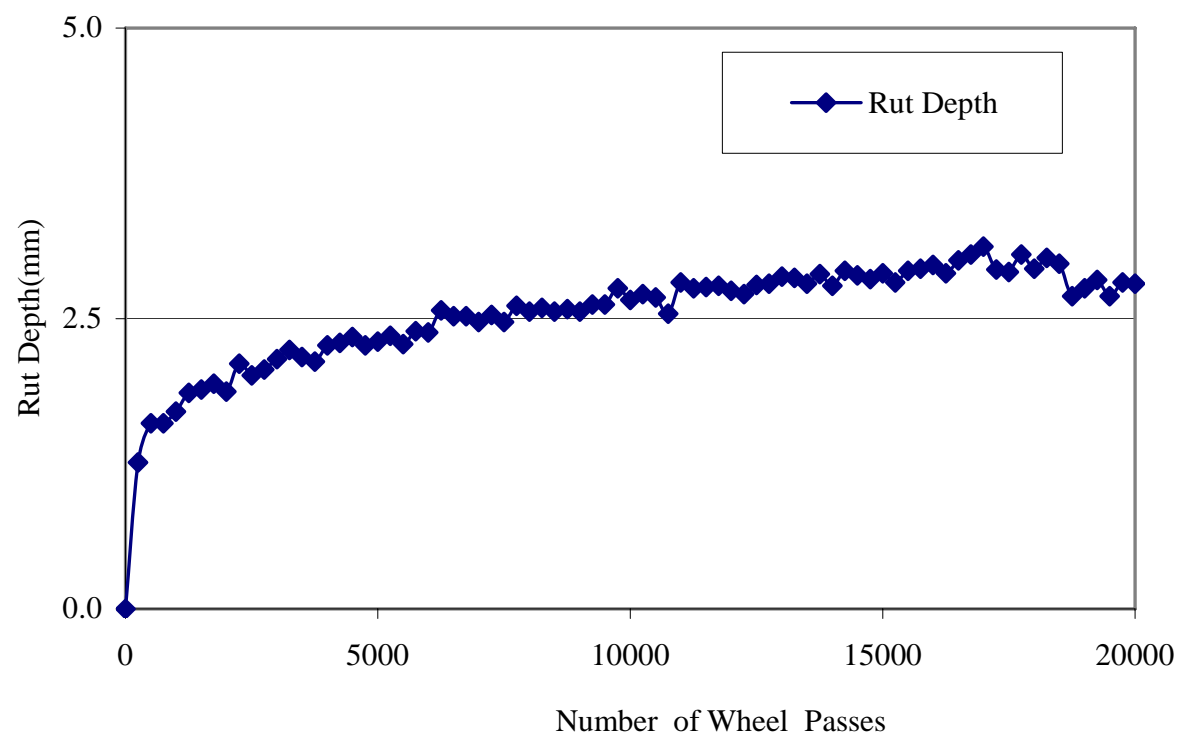

Figure 5-11 Typical PURWheel Rut Data

\section{$\underline{5.3 \text { Dynamic Modulus Test }}$}

\subsubsection{Introduction}

The traditional Marshall and Hveem mixture design methods include a strength test. However, the Superpave mixture design method does not. A simple test to support the volumetric design is needed. One possible method to fill this need is the dynamic modulus test (Witczak et al., 2002). In this study, the dynamic modulus test was used to 
evaluate and predict in-service rutting performance. Table 5-1 lists the advantages and disadvantages associated with this test method.

The dynamic modulus test consists of the application of a haversine compressive stress to a cylindrical specimen that is unconfined as shown in Figure 5-12. Figure 5-13 shows the testing setup used in the dynamic modulus test. Dynamic modulus tests were conducted on NCAT samples as well as specimens fabricated at the Purdue University.

Table 5-1 Advantages and Disadvantages of the Dynamic Modulus Test (Witczak et al., 2002)

\begin{tabular}{|l|l|}
\hline \multicolumn{1}{|c|}{ Advantages } & \multicolumn{1}{c|}{ Disadvantages } \\
\hline $\begin{array}{l}\text { Possible direct application On 2002 Design } \\
\text { Guide }\end{array}$ & $\begin{array}{l}\text { Possible problems with sample } \\
\text { preparation (coring and sawing) }\end{array}$ \\
\hline The need for master curve is eliminated & $\begin{array}{l}\text { Data from the confined tests appear } \\
\text { to be of poor quality }\end{array}$ \\
\hline $\begin{array}{l}\text { Easily related to Witczak regression } \\
\text { equation }\end{array}$ & $\begin{array}{l}\text { Possible mechanical problems with } \\
\text { LVDTs }\end{array}$ \\
\hline $\begin{array}{l}\text { Data available for pavement structural } \\
\text { analysis }\end{array}$ & $\begin{array}{l}\text { Becomes complicated at high } \\
\text { temperatures }\end{array}$ \\
\hline
\end{tabular}

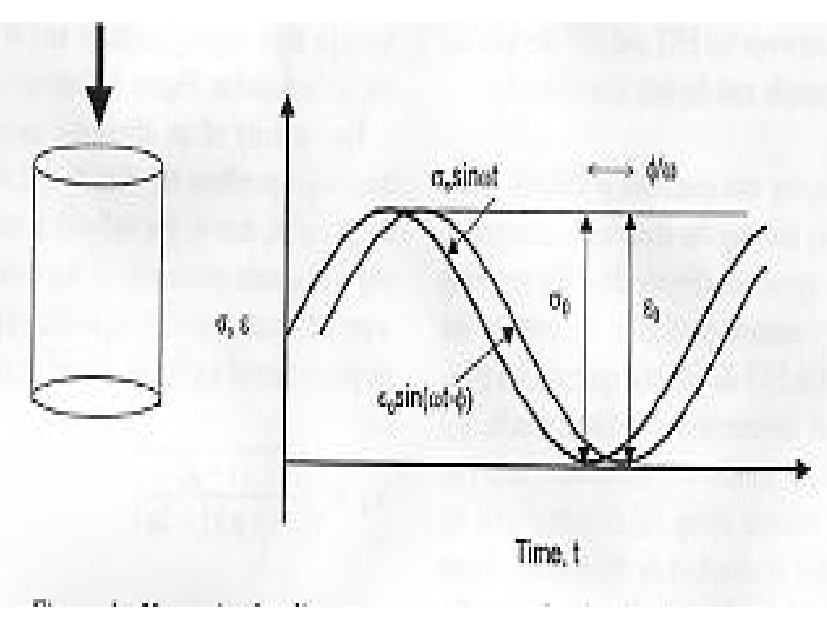

Figure 5-12 Dynamic Modulus Haversine Loading (After Witczak et al., 2002) 


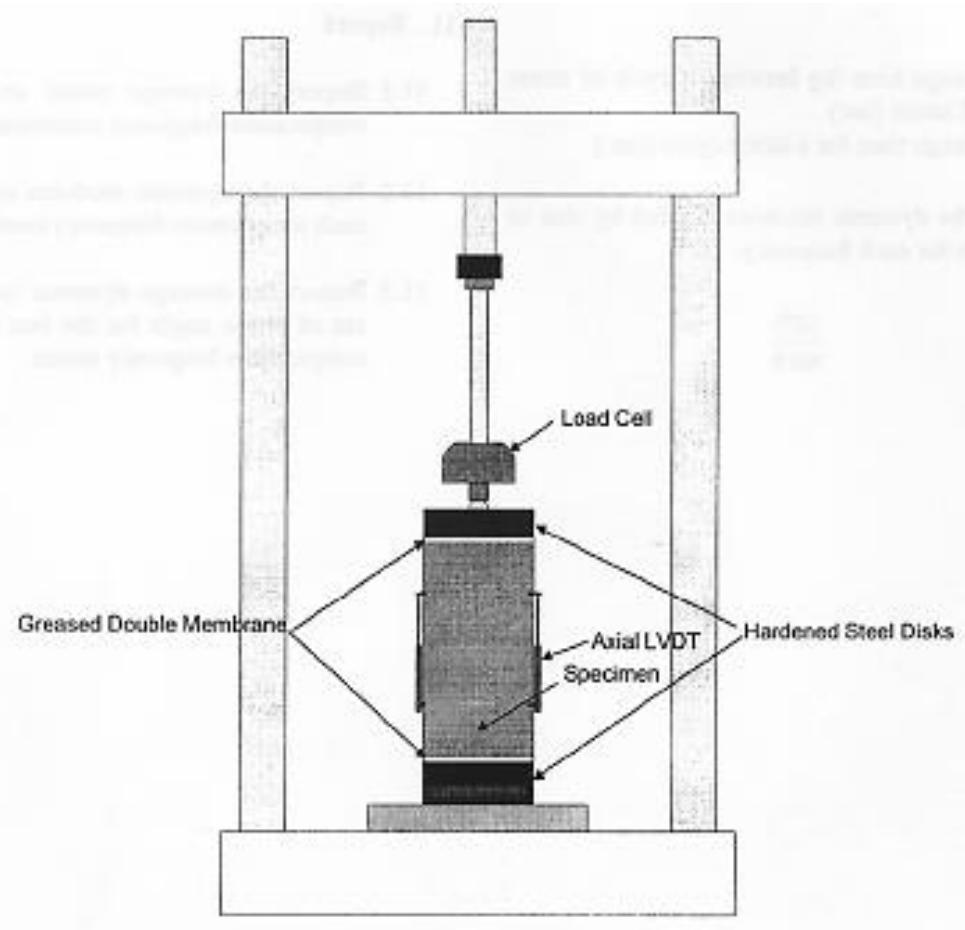

Figure 5-13 Dynamic Modulus Test Configuration (After Witczak et al., 2002)

\subsubsection{NCAT Samples}

Tests on NCAT samples were performed according to the Superpave model draft test guidelines of the NCHRP 9-19 study (referred to as Procedure "X1" at that time). The test temperature was 64C (115F) and conducted on samples compacted to asconstructed air voids. Loose mixture sampled during the construction was compacted using the SGC and reduced to required test size using coring and sawing operations (Powell, 2002).

\subsubsection{APT Samples}

The Heritage Research Group performed the dynamic modulus tests in accordance with the Superpave model draft test guidelines of NCHRP 9-19 study on 
samples prepared at Purdue University. These specimens were fabricated using loose mixture sampled at the paving machine during APT construction. The decision was made to duplicate the in-place air voids of the track mixtures in these specimens so that valid conclusions could be drawn. The $150 \mathrm{~mm}$ (6 in.) diameter specimens were compacted using the SGC and were initially $172 \mathrm{~mm}$ (6.9 in.) tall. They were later reduced to $150 \mathrm{~mm}$ (6 in.) tall by $100 \mathrm{~mm}$ (4 in.) in diameter by coring and sawing operations. Three specimens were fabricated for each mixture tested in the APT. The test temperature was 40C (104F). 


\section{CHAPTER 6 EXPERIMENT TEST RESULTS AND DATA ANALYSIS}

\section{$\underline{6.1 \text { Validation of Superpave Mixture Design Method }}$}

As described in Chapter three, there were five 12.5-mm NMAS mixture designs completed for the test track project. Two contained neat PG 67-22 (N4, N6), two had SBS modified PG 76-22 (N1, N9), and one was made with an SBR modified PG 76-22 (N8). The additional five mixtures placed on the test track were these mixtures with an additional one-half percent binder added.

\subsubsection{Superpave Binder Requirements}

Superpave binder requirements place a minimum limit on the rutting parameter $\mathrm{G}^{*} / \sin \delta$ in an attempt to reduce the rutting potential of an HMA mixture. Figures $6-1$ and 6-2 show the relationship between the Rolling Thin Film Oven (RTFO) aged G*/sin $\delta$ and rutting performance at the NCAT test track and the APT, respectively. Since HMA rutting performance also depends on aggregate gradation and binder content, the comparisons are shown for each factor combination of gradation and binder content. The figures indicate that on average, for both neat and modified binders in the same environmental conditions, that as the $\mathrm{G}^{*} / \sin \delta$ value increases, the HMA mixtures become more resistant to rutting. Finally, as shown in Figure 6-2, the APT data indicates that the $\mathrm{G}^{*} / \sin \delta$ effects are more pronounced for fine-graded mixtures than for coarsegraded mixtures. 


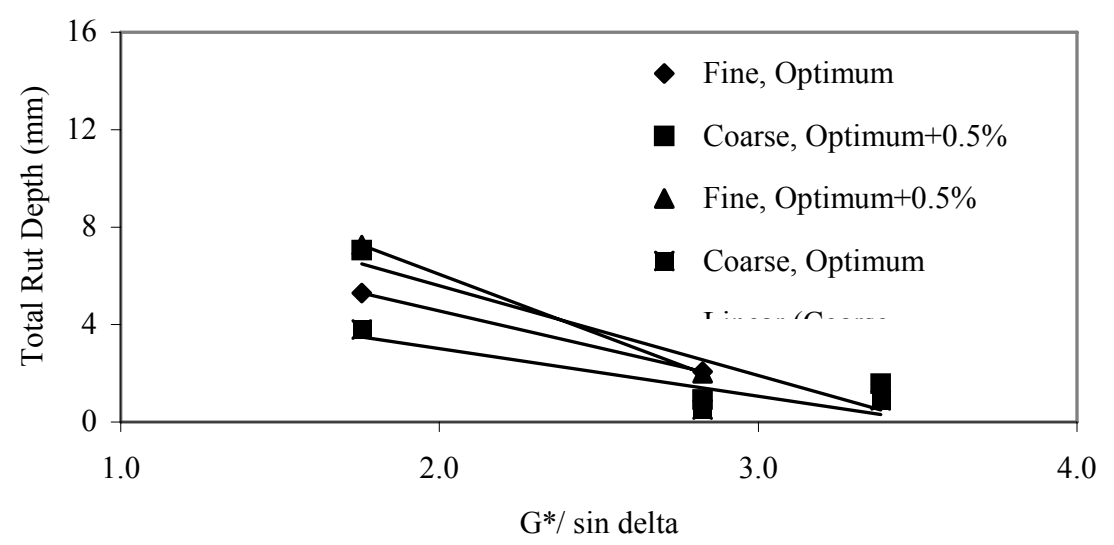

Figure 6-1 Effect of G*/sin $\delta$ on Rutting Performance (NCAT Track)

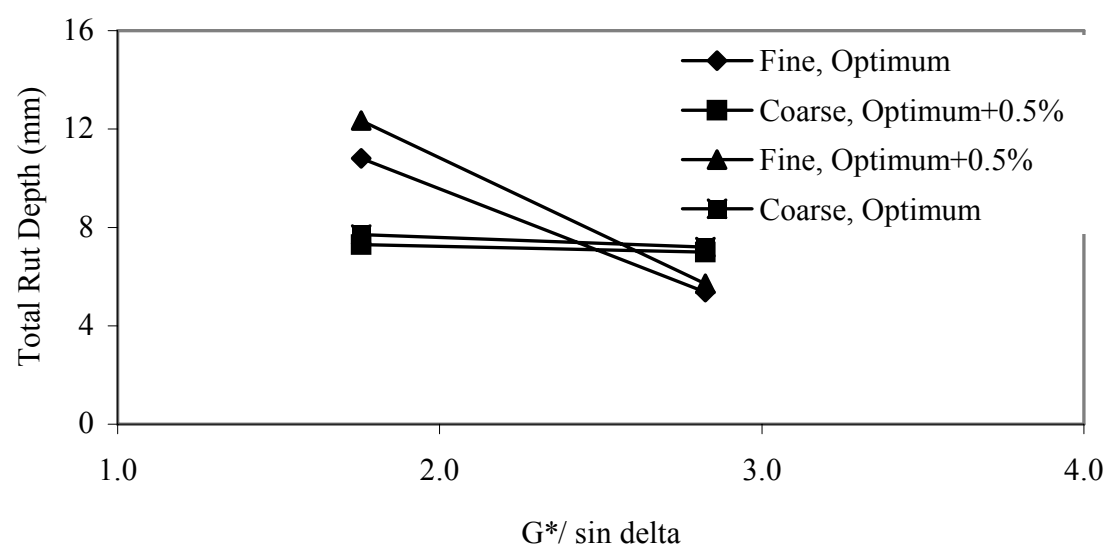

Figure 6-2 Effect of $G^{*} / \sin \delta$ on Rutting Performance (APT)

\subsubsection{Superpave Volumetric Requirements}

\subsubsection{Effect of VMA}

HMA mixtures with low asphalt binder contents can exhibit durability problems due to the thin binder film thickness. A minimum VMA value is specified by the Superpave design method to ensure that sufficient binder is incorporated in a mixture to provide adequate binder film thickness while maintaining enough air voids space to allow for thermal expansion of the binder. For a 12.5-mm NMAS mixture, the minimum 
specified VMA in a compacted HMA mixture is 14 percent. The design VMA data for the test track mixtures is presented in Tables 6-1 along with the final rutting data. It indicates that all mixtures satisfied the Superpave VMA requirement. Figure 6-3 shows the final rut depths as a function of design VMA for the NCAT test track. Since there are only five data points it is hard to draw any firm conclusions. It does appear that the slope of the best fit line for the modified binder mixtures is slightly steeper than the line for the neat mixtures.

Table 6-1 NCAT Test Track Mixture Design Properties and Rutting Data

\begin{tabular}{|c|c|c|c|c|c|c|}
\hline Section & $\begin{array}{c}\text { Binder } \\
\text { Content (\%) }\end{array}$ & $\begin{array}{c}\text { Air Voids } \\
(\%)\end{array}$ & $\begin{array}{c}\text { VMA } \\
(\%)\end{array}$ & $\begin{array}{c}\text { VFA } \\
(\%)\end{array}$ & $\begin{array}{c}\text { Dust } \\
\text { Proportion }\end{array}$ & $\begin{array}{c}\text { Total } \\
\text { Rut (mm) }\end{array}$ \\
\hline N1 & 7.5 & 4.0 & 15.8 & 74.7 & 1.0 & 2.1 \\
\hline N4 & 7.9 & 4.0 & 16.5 & 75.8 & 0.9 & 5.3 \\
\hline N6 & 7.7 & 4.0 & 14.8 & 73.0 & 1.3 & 3.8 \\
\hline N8 & 7.7 & 4.0 & 15.1 & 73.5 & 1.3 & 0.9 \\
\hline N9 & 7.3 & 4.0 & 14.8 & 73.0 & 1.3 & 0.5 \\
\hline
\end{tabular}

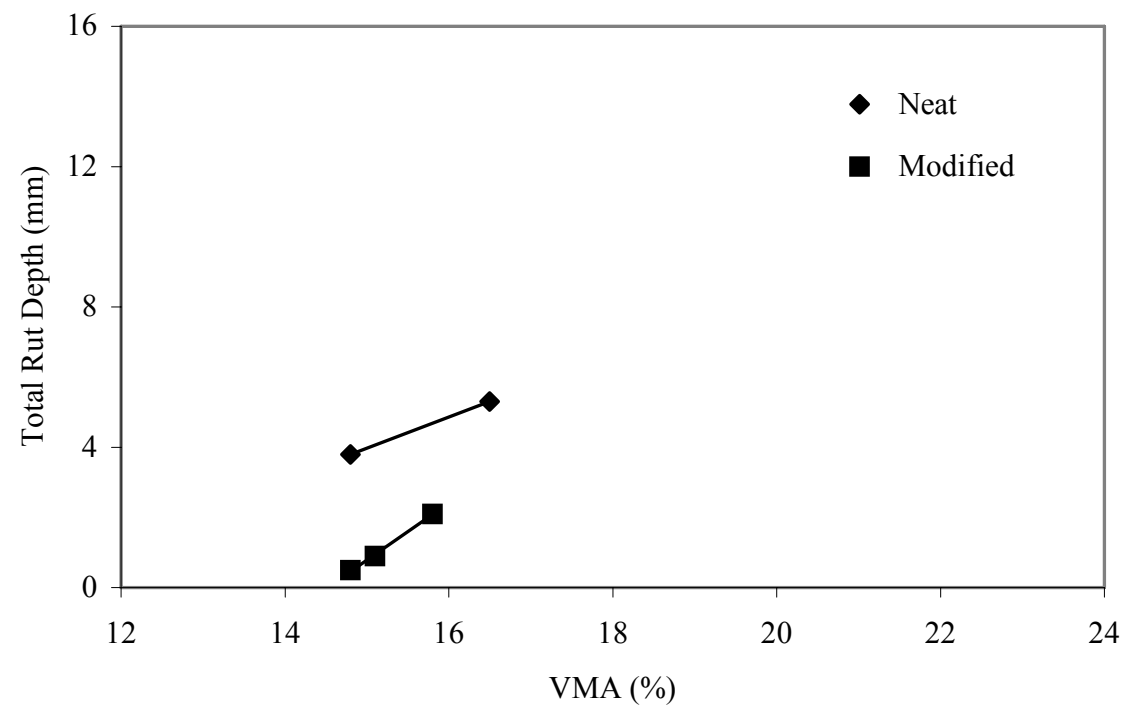

Figure 6-3 Effect of Design VMA on Rutting Performance (NCAT Track) 


\subsubsection{Effect of VFA}

Superpave specifications require a VFA in the range of 65-78 percent for the design traffic level used in the study. The data in Table 6-1 indicates that design mixtures of the NCAT test track satisfied the Superpave VFA requirement. Figure 6-4 shows the rut depth as a function of the design VFA for the NCAT test track. Again the modified binder mixtures appear to have a slightly steeper slope than does the line for the neat binder mixtures. This might indicate that the modified mixtures are a bit more sensitive, but with such a small data set, this cannot be concluded.

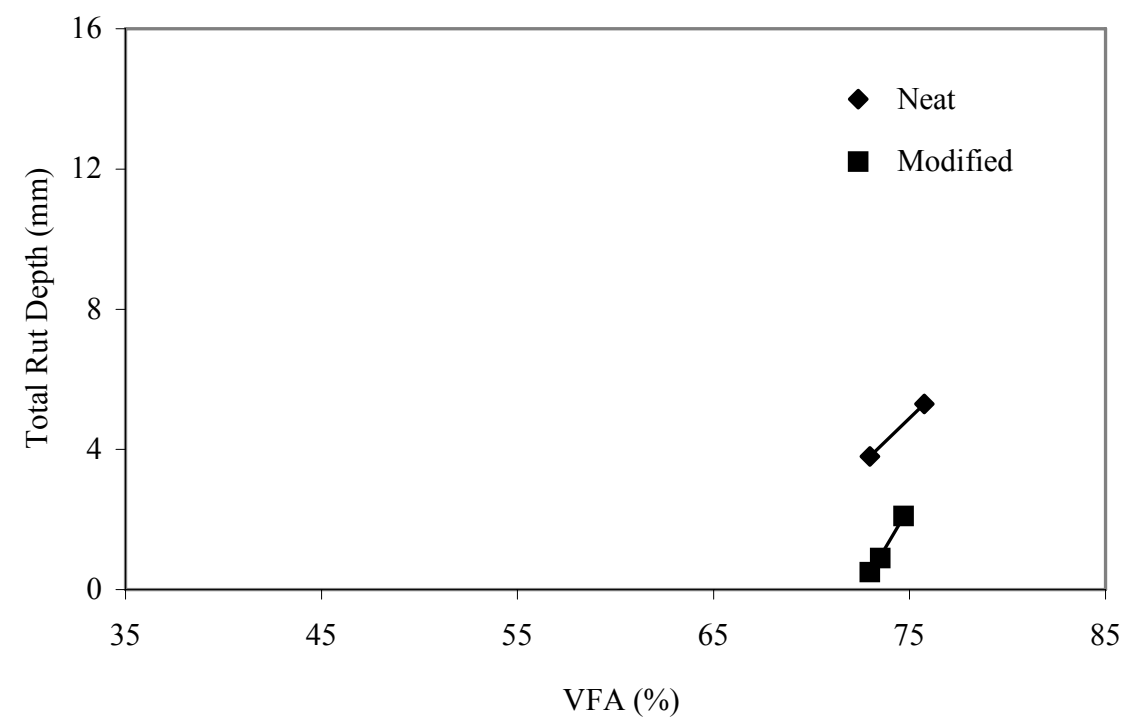

Figure 6-4 Effect of Design VFA on Rutting Performance (NCAT Track)

\subsubsection{Effect of Dust Proportion}

The dust proportion specification was implemented with the Superpave mixture design method to ensure that a mixture has adequate quantities of filler material (smaller than the $0.075-\mathrm{mm}$ (No. 200) sieve). Superpave specifications require a dust proportion in the range of 0.6 and 1.6. The data in Table 6-1 indicates that design mixtures satisfied 
the dust proportion requirement. Figure 6-5 shows the rut depth as a function of dust proportion. As the dust proportion increases, the rut depths observed at the test track decreases. This seems logical since aggregate dust can act to produce a stiffer mastic, resulting in better rutting resistance.

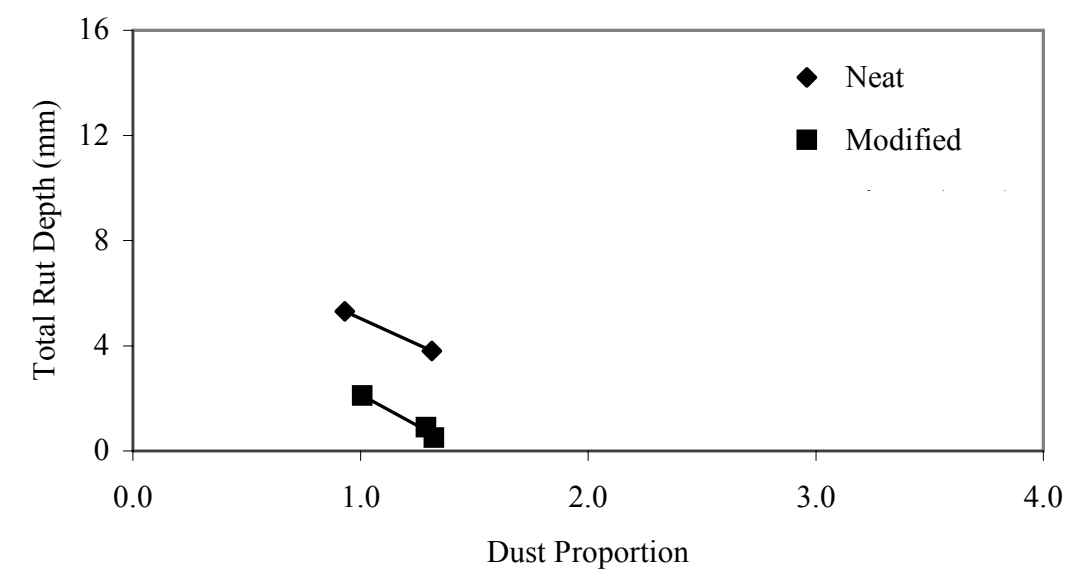

Figure 6-5 Effect of Design Dust Proportion on Rutting Performance (NCAT Track)

\subsubsection{Summary}

The HMA mixtures tested in the study were designed using the Superpave mixture design method and the designed mixtures did satisfy the specifications for VMA, VFA and dust proportion. After the application of 10 million ESAL at the NCAT test track, all of mixtures, even those with higher than optimum binder content, exhibited good rutting resistance (Table 6-1 and Table 6-2) with N3 exhibiting the highest rut depth and N9 the lowest. These findings are consistent with the experimental design where the section N3 was designed to exhibit the highest rutting potential and N9 the lowest. It 
appears that when quality materials and construction techniques are used, the Superpave mixture design method can produce high quality HMA mixtures.

Table 6-2 NCAT Test Track As-Constructed Mixture Properties and Rutting Data

\begin{tabular}{|c|c|c|c|c|c|c|c|}
\hline \multirow[b]{2}{*}{ Section } & \multicolumn{6}{|c|}{ Track } & \multirow{2}{*}{$\begin{array}{c}\text { PURWheel } \\
\text { Total } \\
\text { Rut (mm) }\end{array}$} \\
\hline & $\begin{array}{c}\text { Binder } \\
\text { Content }(\%)\end{array}$ & $\begin{array}{c}\text { Air Voids } \\
(\%)\end{array}$ & $\begin{array}{c}\text { VMA } \\
(\%)\end{array}$ & $\begin{array}{l}\text { VFA } \\
(\%)\end{array}$ & $\begin{array}{c}\text { Dust } \\
\text { Proportion }\end{array}$ & $\begin{array}{c}\text { Total } \\
\text { Rut } \\
\text { (mm) }\end{array}$ & \\
\hline N1 & 7.4 & 4.9 & 17.0 & 71.2 & 1.2 & 2.1 & 2.3 \\
\hline $\mathrm{N} 2$ & 7.8 & 5.3 & 18.2 & 70.8 & 1.3 & 2.0 & 3.0 \\
\hline N3 & 7.6 & 5.9 & 17.9 & 67.0 & 1.2 & 7.3 & 6.2 \\
\hline N4 & 6.8 & 6.6 & 16.7 & 60.6 & 1.3 & 5.3 & 4.4 \\
\hline N5 & 6.8 & 6.2 & 15.2 & 59.3 & 2.0 & 7.1 & 3.5 \\
\hline N6 & 6.8 & 5.6 & 15.0 & 62.5 & 1.9 & 3.8 & 3.4 \\
\hline N7 & 6.9 & 6.1 & 16.1 & 62.2 & 1.7 & 1.6 & 2.3 \\
\hline N8 & 6.6 & 5.3 & 14.4 & 63.2 & 1.8 & 0.9 & 2.4 \\
\hline N9 & 6.7 & 5.5 & 14.6 & 62.2 & 2.1 & 0.5 & -1 \\
\hline N10 & 6.8 & 5.3 & 15.0 & 64.7 & 1.7 & 0.9 & -1 \\
\hline
\end{tabular}

${ }^{1}$ These mixtures were not tested in the PURWheel due to sample degradation.

\section{$\underline{6.2 \text { Effect of As-constructed Volumetrics on Rutting Performance }}$}

\subsubsection{Effect of VMA}

The as-constructed VMA data for the test track and the APT are presented in Tables 6-2 and 6-3, respectively along with the final rutting data for both test venues. Figures 6-6 and 6-7 show the final rut depths as a function of in-place VMA for the NCAT test track and the APT, respectively. It appears that the test track was more sensitive to VMA changes than was the APT. This is certainly true for modified binders. Note that for the modified binders in the APT, the relationship indicates hat rutting decreases with increasing VMA. This is opposite the trend for the neat, and for both the 
neat and modified mixtures on the test track. The reverse slope is likely due to low densities in the APT for the modified mixtures.

Table 6-3 APT As-Constructed Mixture Properties and Rutting Data

\begin{tabular}{|c|c|c|c|c|c|c|c|}
\hline \multirow[b]{2}{*}{ Section } & \multicolumn{6}{|c|}{ APT } & \multirow{2}{*}{\begin{tabular}{|c} 
PURWheel \\
Total \\
Rut (mm) \\
\end{tabular}} \\
\hline & $\begin{array}{c}\text { Binder } \\
\text { Content }(\%)\end{array}$ & $\begin{array}{c}\text { Air Voids } \\
(\%)\end{array}$ & $\begin{array}{c}\text { VMA } \\
(\%)\end{array}$ & $\begin{array}{l}\text { VFA } \\
(\%)\end{array}$ & $\begin{array}{c}\text { Dust } \\
\text { Proportion }\end{array}$ & $\begin{array}{c}\text { Total } \\
\text { Rut }(\mathrm{mm}) \\
\end{array}$ & \\
\hline N1 & 7.4 & 8.3 & 19.3 & 56.9 & 1.7 & 5.4 & 4.5 \\
\hline $\mathrm{N} 2$ & 7.7 & 13.1 & 23.7 & 44.7 & 1.7 & 5.7 & 6.5 \\
\hline N3 & 7.5 & 10.6 & 20.2 & 47.6 & 1.2 & 12.3 & 19 \\
\hline $\mathrm{N} 4$ & 6.9 & 9.9 & 19.3 & 48.8 & 1.5 & 10.8 & 17.5 \\
\hline N5 & 7.0 & 12.7 & 19.8 & 35.9 & 2.0 & 7.3 & 5.6 \\
\hline N6 & 6.5 & 10.7 & 18.0 & 40.6 & 2.4 & 7.7 & 5.6 \\
\hline \multicolumn{8}{|l|}{$\mathrm{N} 7^{1}$} \\
\hline \multicolumn{8}{|l|}{$\mathrm{N} 8^{1}$} \\
\hline N9 & 6.5 & 9.2 & 15.9 & 42.0 & 2.3 & 7.2 & 6.9 \\
\hline N10 & 7.0 & 4.3 & 12.4 & 65.2 & 2.3 & 7.0 & 6 \\
\hline
\end{tabular}

${ }^{1}$ These mixtures were not produced in the APT.

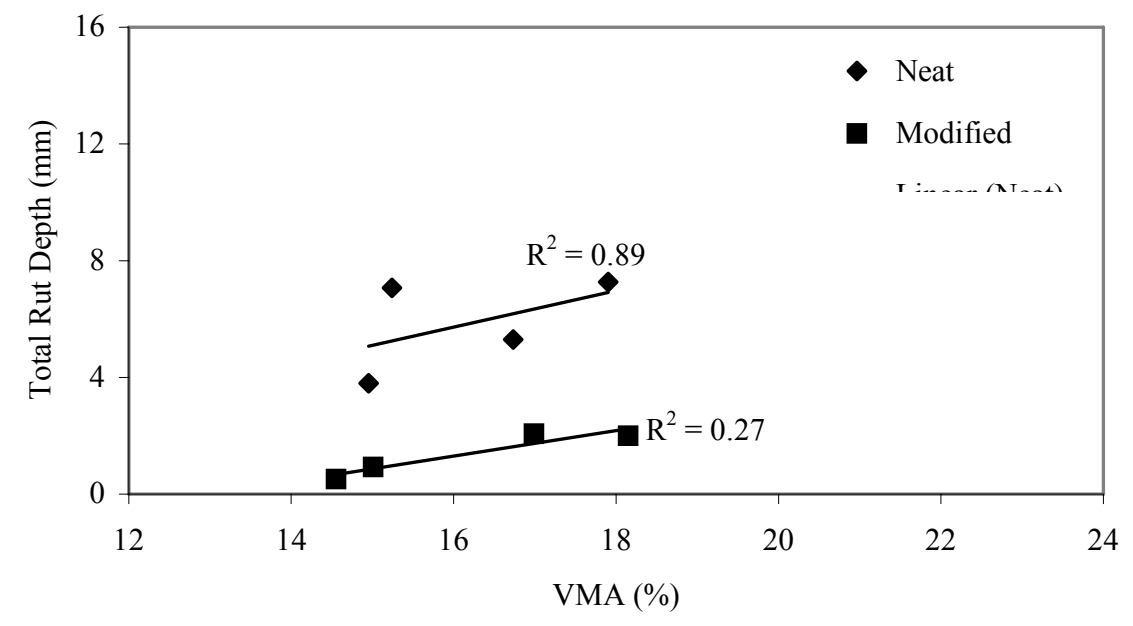

Figure 6-6 Effect of As-Constructed VMA on Rutting Performance (NCAT Track) 


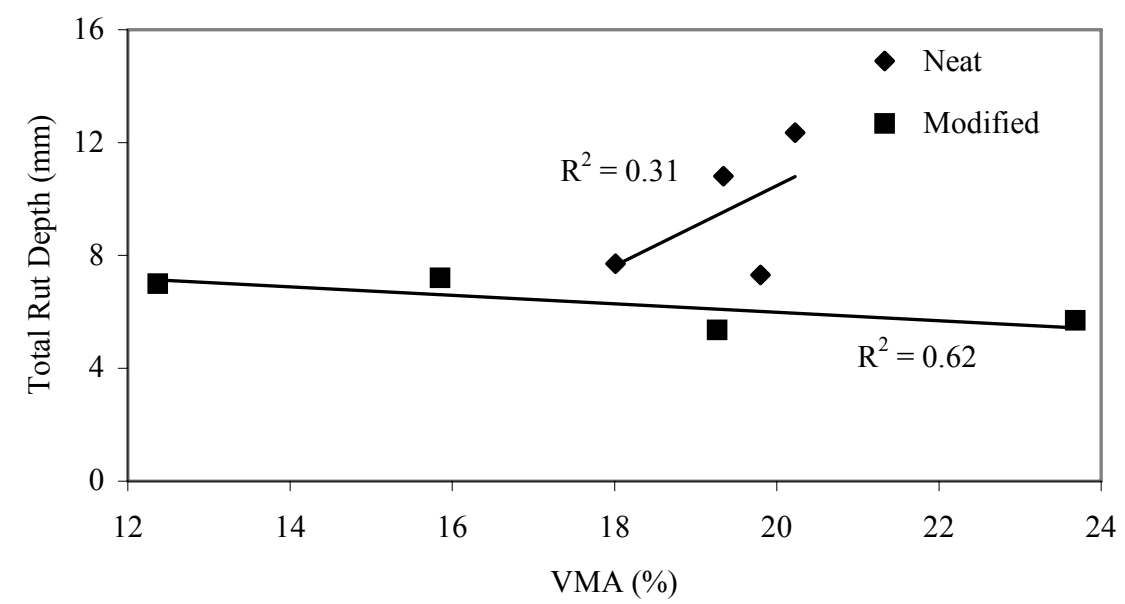

Figure 6-7 Effect of As-Constructed VMA on Rutting Performance (APT)

\subsubsection{Effect of VFA}

Figures 6-8 and 6-9 show the rut depth as a function of the as-constructed VFA for the NCAT test track and the APT, respectively. Again the track appears more sensitive to VFA than the APT for modified binders. The poor data for modified mixtures in the APT is again likely due to the low density problem.

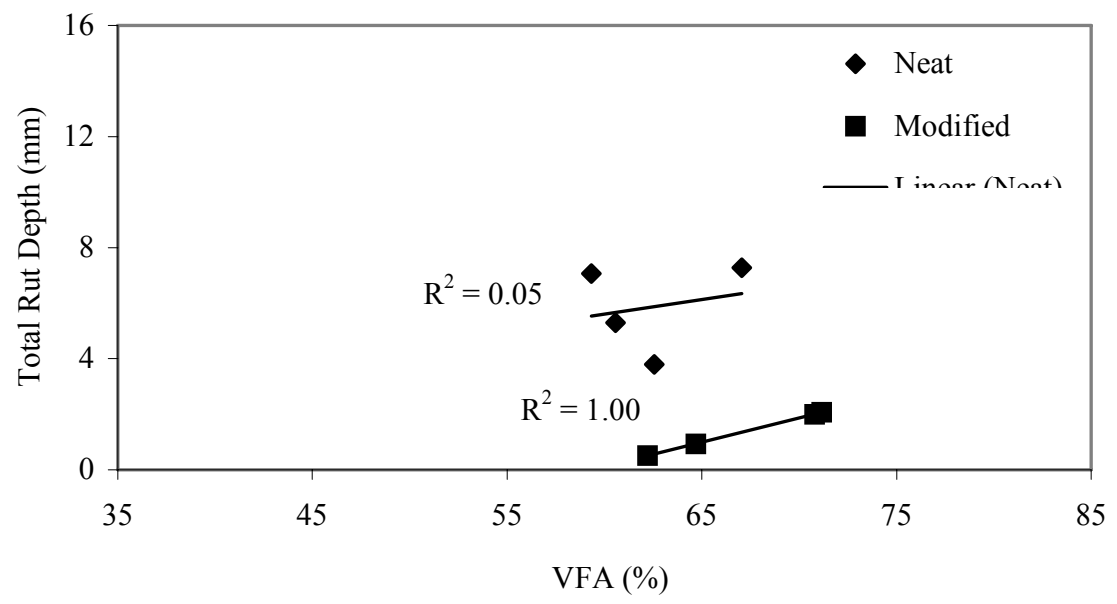

Figure 6-8 Effect of As-Constructed VFA on Rutting Performance (NCAT Track) 


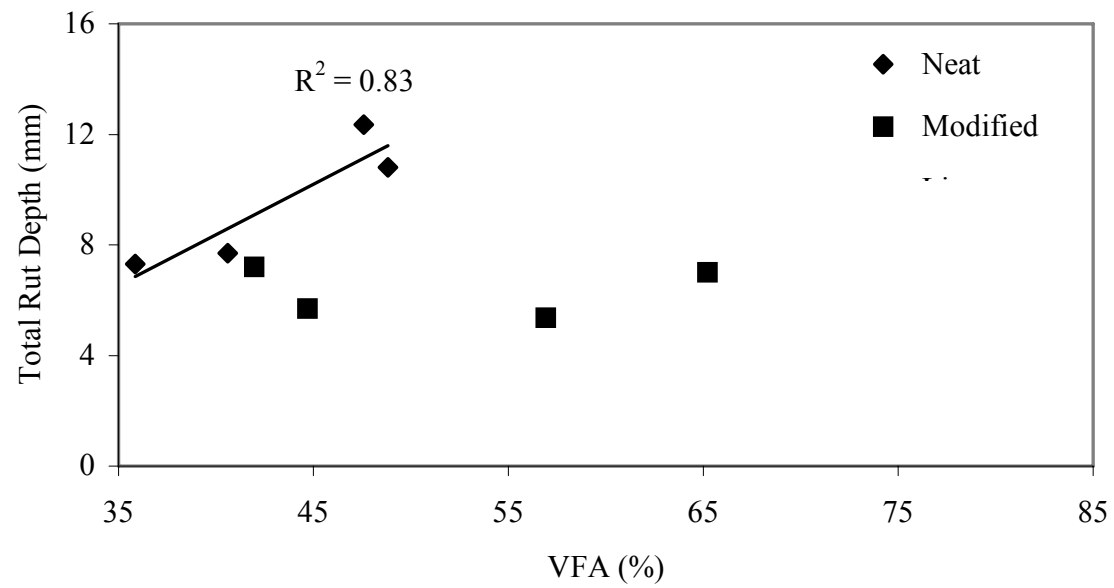

Figure 6-9 Effect of As-Constructed VFA on Rutting Performance (APT)

\subsubsection{Effect of Dust Proportion}

Figures 6-10 and 6-11 show the rut depth as a function of dust proportion. In general, as the dust proportion increases, the rut depths observed at both the test track and in the APT decrease except for the modified binder mixtures in the APT (low densities).

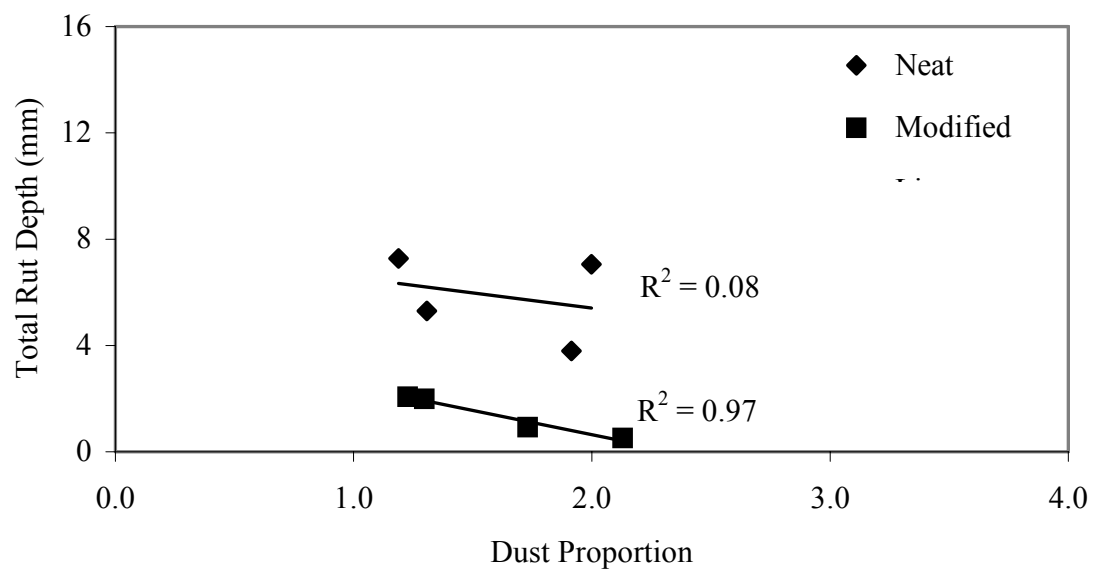

Figure 6-10 Effect of As-Constructed Dust Proportion on Rutting Performance (NCAT Track) 


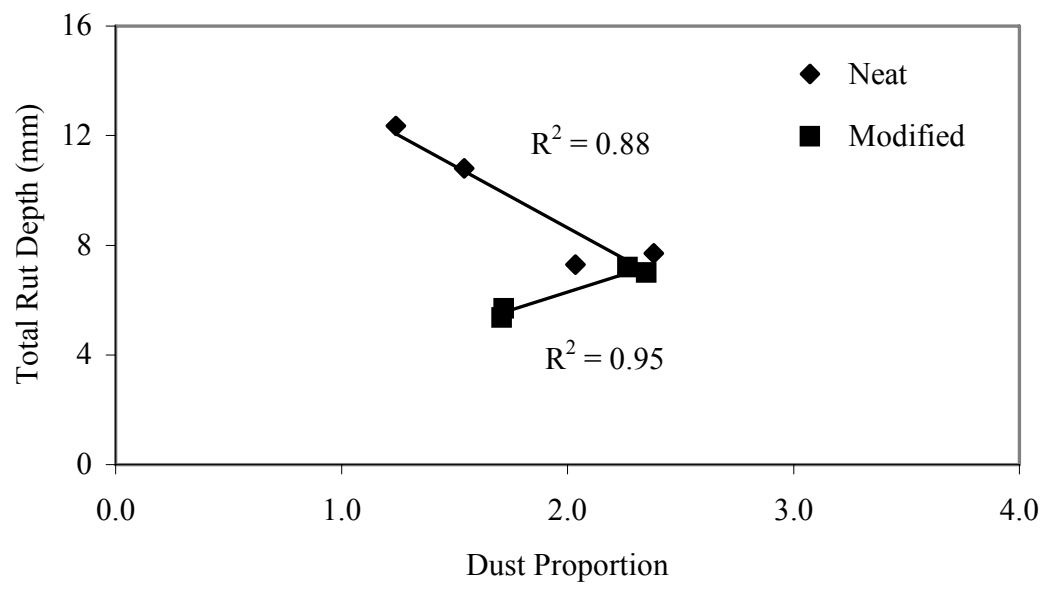

Figure 6-11 Effect of As-Constructed Dust Proportion on Rutting Performance (APT)

\subsubsection{Effect of Binder Film Thickness}

Mixtures with thick binder films can have reduced inter-particle contact and therefore lower internal friction to resist traffic loads and lower rutting resistance. If the film is too thin, the mixture may be prone to moisture damage and/or oxidation and lower shear strength and lower rutting resistance. Stiady (2000) indicated that binder film thickness is a good indicator of an HMA mixture's ability to resist rutting. His work suggests that a binder film thickness of 7 to 9 microns is optimal to improve rutting resistance. Figures 6-12 and 6-13 show the rut depths experienced by the mixtures in this study as a function of their as-constructed binder film thickness for the NCAT test track and APT sections, respectively. The data indicate that rutting increases as binder film thickness increases, but none of the mixture had extremely high binder films. All of the mixtures appear to have given good in-service performance. 


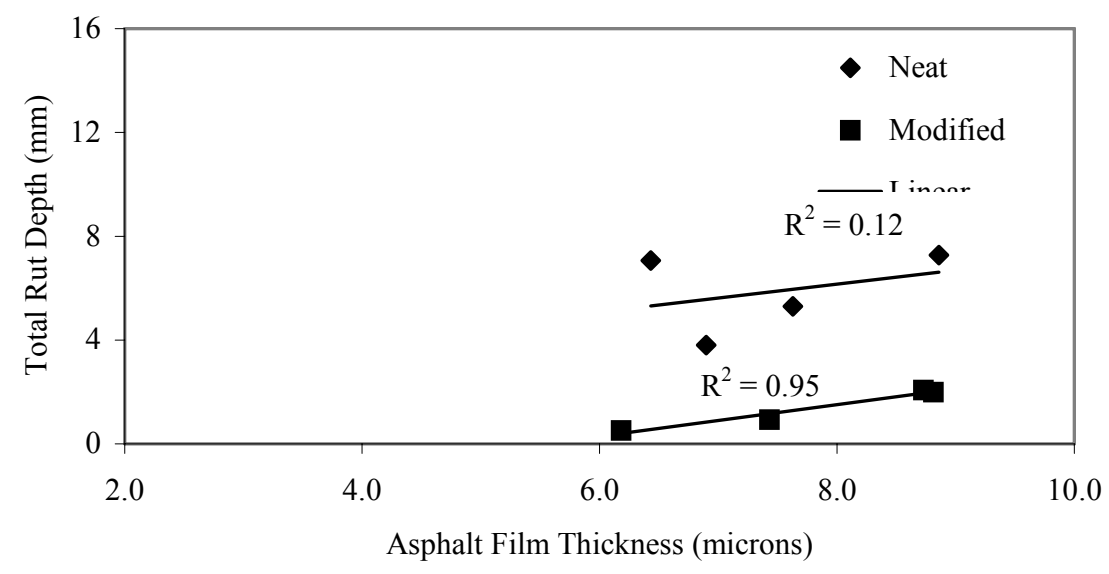

Figure 6-12 Effect of As-Constructed Asphalt Film Thickness on Rutting Performance (NCAT Track)

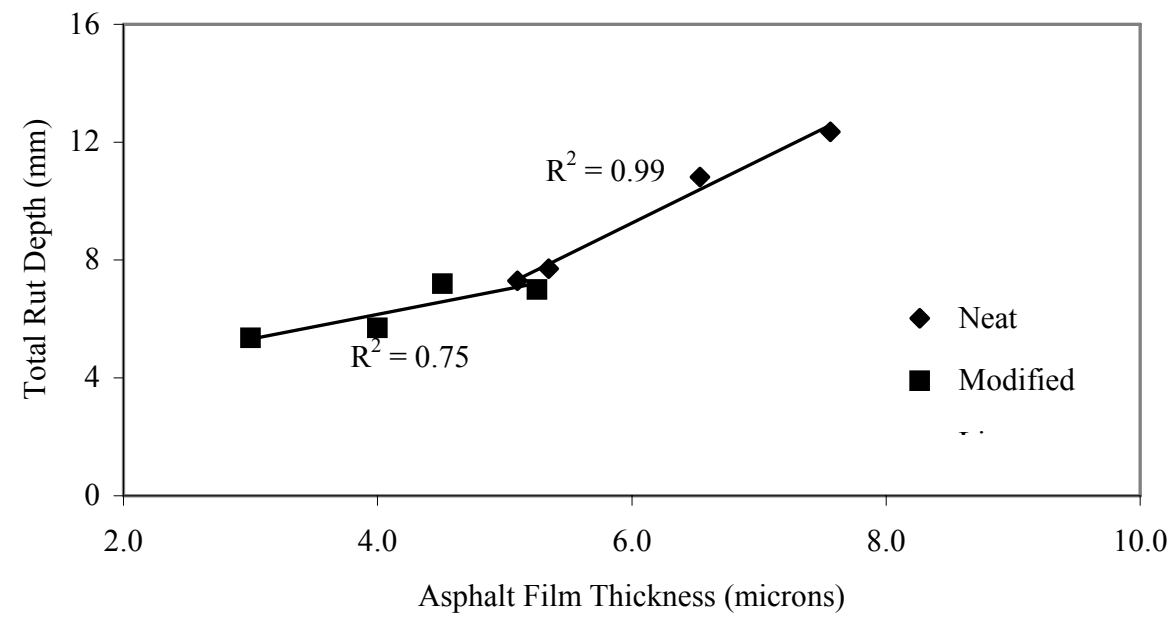

Figure 6-13 Effect of As-Constructed Asphalt Film Thickness on Rutting Performance (APT)

\section{$\underline{6.3 \text { Statistical Analysis of Rutting Data }}$}

A statistical analysis was conducted to study the effect of each experimental factor. The original experimental study included three factors: aggregate gradation, 
binder type, and binder content. A three-way analysis of variance (ANOVA) was

conducted using the Statistical Analysis System (SAS). Each factor had two levels, which resulted in a total of $8(2 \times 2 \times 2)$ factor level combinations. The response variable was total rut depth. An initial linear regression model that included all factor level combinations was assumed in the first model. The results of this first attempt were used to eliminate non-significant term(s) in the model and the refined model was tested for statistical significance. This procedure was repeated until all terms in the model were significant. The results are shown in the upper half of Table 6-4.

Table 6-4 Statistical Analysis of Total Rut Data

\begin{tabular}{|c|c|c|c|}
\hline $\begin{array}{l}\text { Test } \\
\text { Method }\end{array}$ & \multicolumn{3}{|c|}{ Significant Factors (Factors and/or Combinations) } \\
\hline Track & \multicolumn{3}{|c|}{ Gradation, Binder Type, Binder Content, Binder Content $\times$ Binder Type } \\
\hline APT & \multicolumn{3}{|c|}{ Gradation, Binder Type, Gradation $\times$ Binder Type } \\
\hline PURWheel & \multirow{2}{*}{\multicolumn{3}{|c|}{ Gradation, Binder Type, Binder Content, Gradation $\times$ Binder Type }} \\
\hline & & & \\
\hline $\begin{array}{c}\text { Test } \\
\text { Method }\end{array}$ & Fine vs. Coarse & Neat vs. Modified & $\begin{array}{l}\text { Optimum Binder Content vs. } \\
\text { Optimum Plus Binder Content }\end{array}$ \\
\hline Track & Yes $^{1}$ & Yes & Yes \\
\hline APT & Yes & Yes & Yes \\
\hline PURWheel & Yes & Yes & Yes \\
\hline
\end{tabular}

${ }^{1}$ Indicates that the comparison factor levels are significantly different.

The results suggest that the three main factors do indeed have significant effects on the rutting performance of the HMA mixtures tested. However, for the APT, the data indicates that binder content does not significantly affect the rutting performance. This lack of statistical significance could be due to the larger differences in the initial densities; the initial density was not included as a factor in the experimental design. In addition to the main factors, there is one significant interaction effect for each of the three 
test methods. For the test track it is the interaction of binder content and binder type. For the APT and PURWheel it is the interaction of gradation and binder type. The APT data reveals that modified binders improve rutting performance better in fine-graded mixtures than in coarse-graded mixtures. The test track performance data indicate that when modified binders were used, they were able to compensate for over-asphalting of the mixtures.

A mean response comparison study also was conducted for each factor using the SAS program. It was done in order to answer the questions:

1. Do HMA mixtures with optimum and optimum plus one-half percent binder contents have equal rutting resistances?

2. Do HMA mixtures with neat and modified binders have equal rutting resistances?

3. Do fine- and coarse-graded HMA mixtures have equal rutting resistances? The results are presented in the lower half of Table 6-4. They reveal that each factor level of gradation, binder type, and binder content are statistically different. Thus it appears that HMA mixtures with optimum and optimum plus one-half percent do not have equal rutting performance. Likewise, neat and modified binders do not have equal rutting performance nor do fine- and coarse-graded HMA mixtures.

\subsection{Effect of Mixture Properties on Rutting Performance}

As illustrated in Chapter 2, the rutting performance of an HMA mixture is dependent on various mixture properties. This subsection explains the HMA rutting behavior as it is related to the mixture properties. 


\subsubsection{Effect of Binder Content}

Figures 6-14 and Figure 6-15 show the effects of binder content on the rutting performance of the test track and the APT, respectively. It is evident from the test track data that, in general, increasing the binder content resulted in higher rutting potential and that the effect is more pronounced for the neat binder than for the modified binders. This is confirmed through the statistical analysis (section 6.3) with the existence of the interaction between the binder content and type. It appears that slightly higher binder contents can be allowed with modified binders without significantly affecting the rutting performance. This could have the advantage of improving mixture durability.

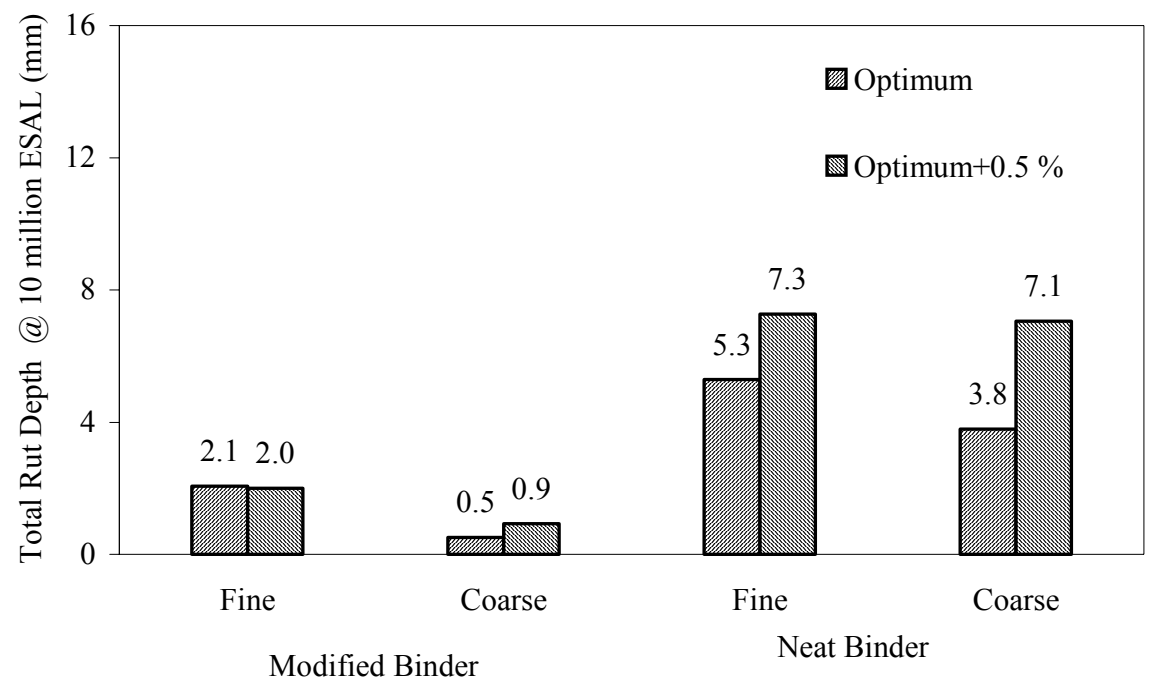

Figure 6-14 Effect of Binder Content on NCAT Track Rutting Performance

Also, for the APT data, note the comparable amounts of rutting in the coarsegraded mixtures having optimum and optimum plus one-half percent binder contents. The initial density of the optimum binder content mixture (N9) was 90.8 percent. The optimum plus one-half percent binder content mixture (N10) had an initial density of 95.7 
percent. The relatively low initial in-place density of the optimum binder content mixture may be the reason the mixture experienced rutting similar to the optimum plus one-half percent binder content mixture.

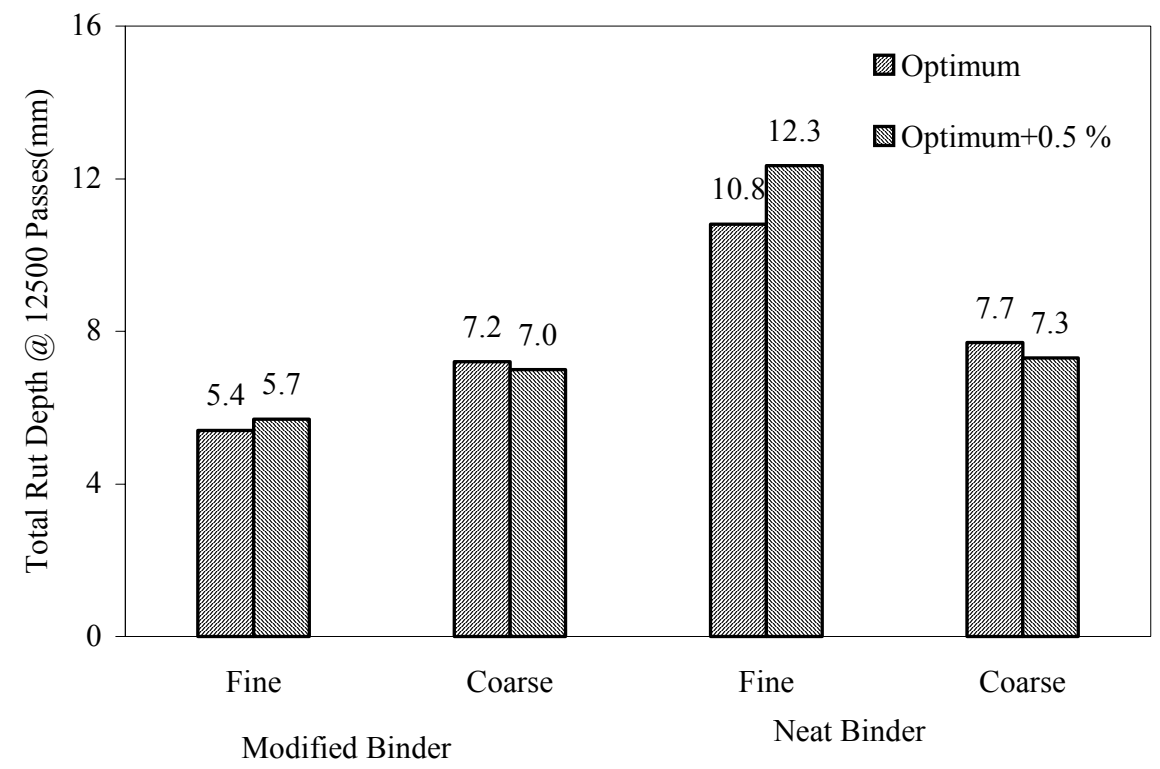

Figure 6-15 Effect of Binder Content on APT Rutting Performance

\subsubsection{Effect of Binder Type}

Figures 6-16 and 6-17 show the effects of the binder type on rutting performance for the test track and APT, respectively. The plots indicate that mixtures with modified binders had improved rutting resistance over the mixtures containing neat binders. This observation confirms the potential gains in using modified binders for a given application. The improved behavior is similar for the test track mixtures regardless of the gradation type. However, the APT data reveals that improvement in rutting performance by the modified binders is higher for the fine-graded mixtures than for the coarse-graded 
mixtures. This is confirmed through the statistical analysis with the existence of the interaction between binder type and gradation for the APT mixtures.

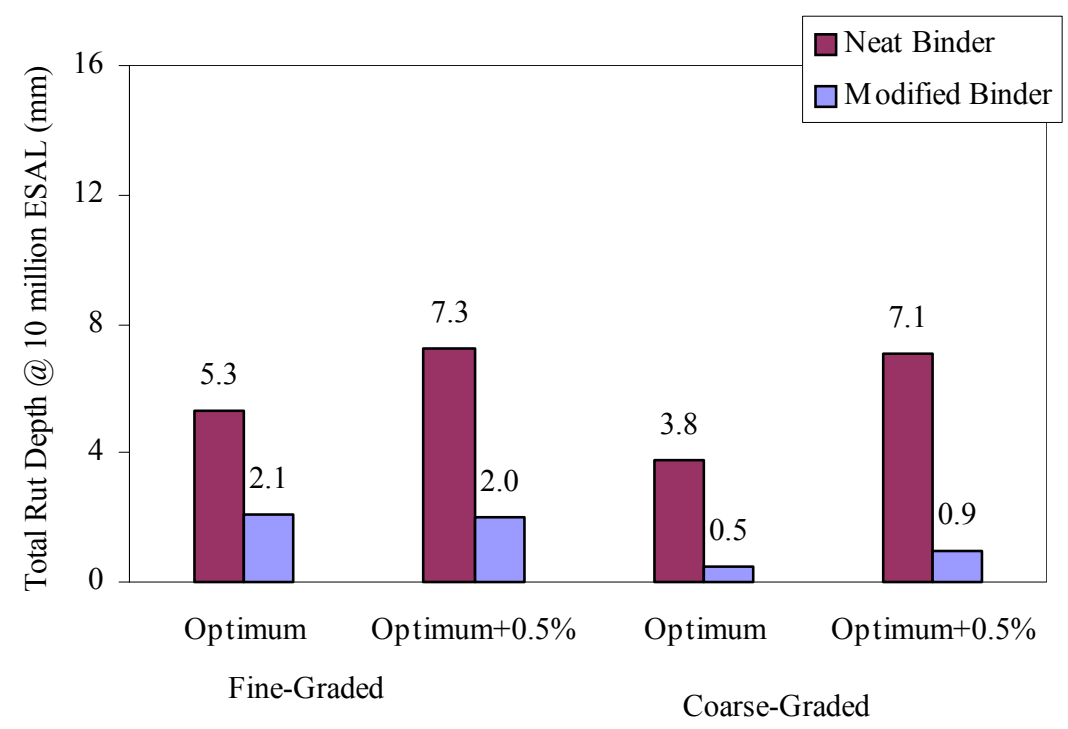

Figure 6-16 Effect of Binder Type on NCAT Track Rutting Performance

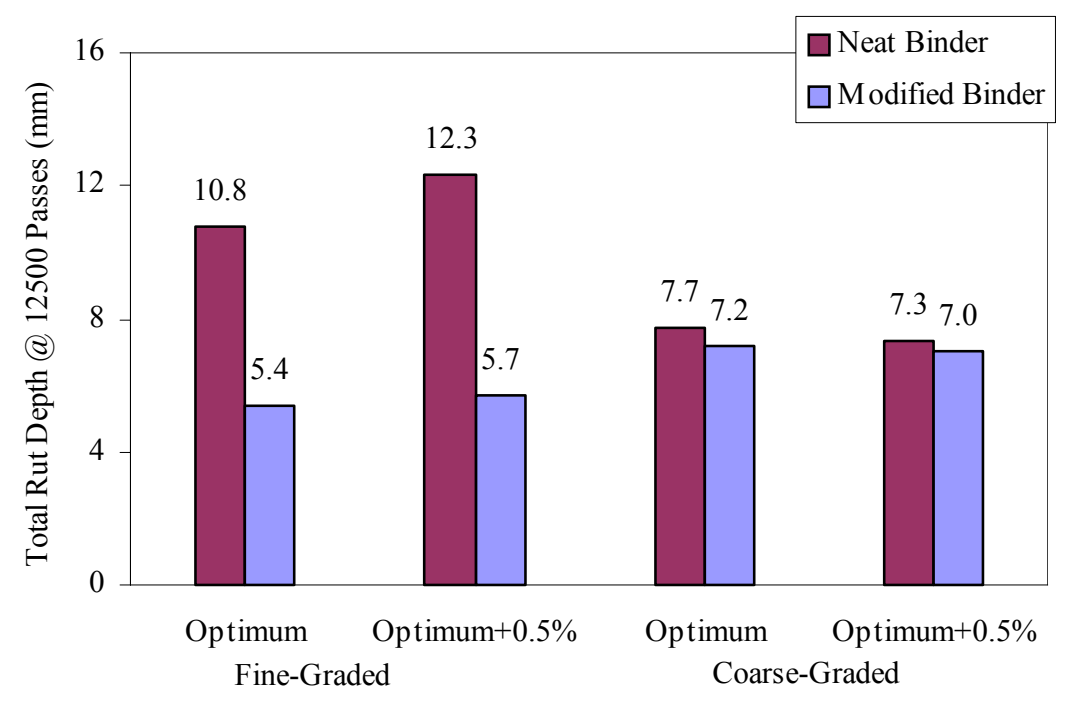

Figure 6-17 Effect of Binder Type on APT Rutting Performance 


\subsubsection{Effect of Aggregate Gradation}

Figures 6-18 and 6-19 illustrate the effects of aggregate gradation on the rutting performance at the test track and in the APT, respectively. The plots show that, in general, coarse-graded mixtures have slightly improved rutting resistance compared to fine-graded mixtures. However, the rutting performance of the fine-graded mixtures appears to be within the acceptable range (less than $6.25 \mathrm{~mm}(0.25 \mathrm{in}$.$) ) except for the$ mixtures containing neat binders at optimum plus one-half percent binder contents (N3 and N5). In the APT, the fine-graded mixtures containing modified binders exhibited slightly better rutting resistance than the coarse-graded mixtures containing modified binders. As the APT tests were conducted at an elevated constant temperature of 50C (122F) and the initial densities were similar (91.7 and 90.8\% for N1, and N9 respectively), the results seem to suggest that fine-graded mixtures can be used for heavy traffic applications if designed and placed correctly.

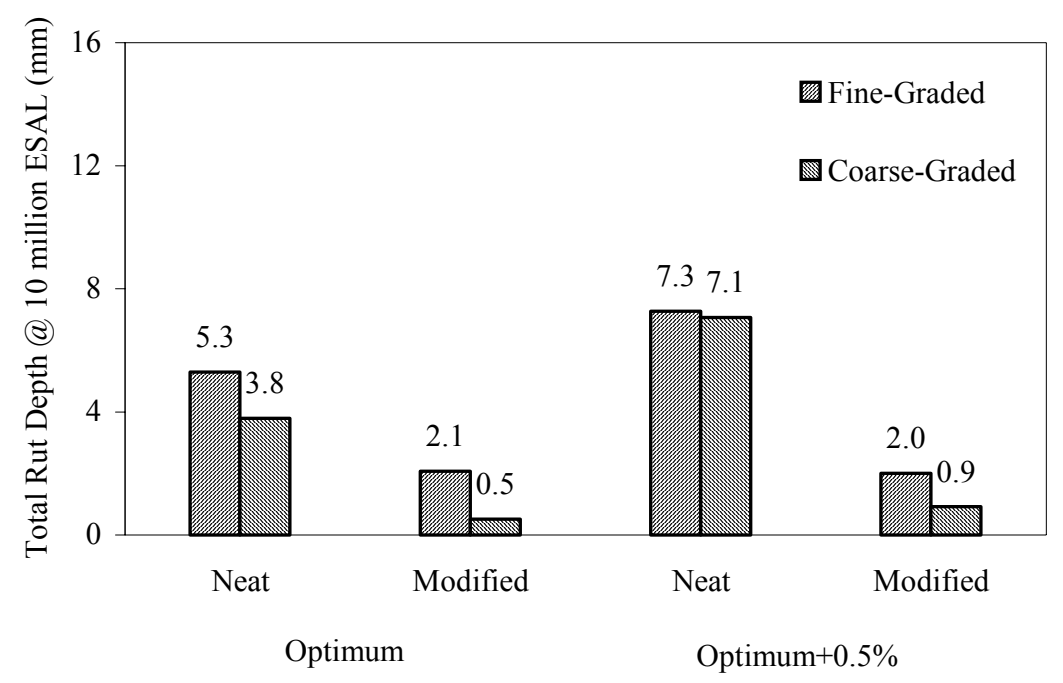

Figure 6-18 Effect of Aggregate Gradation on NCAT Track Rutting Performance 


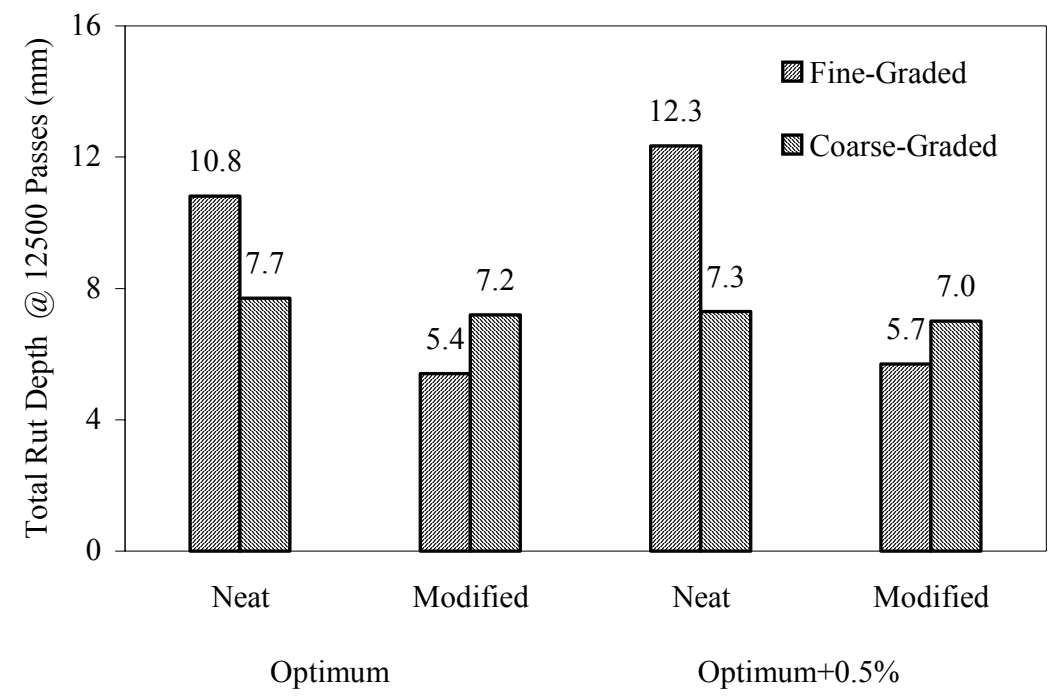

Figure 6-19 Effect of Aggregate Gradation on APT Rutting Performance

\subsubsection{Effect of Initial Density}

Another property that can play an important role in determining the rutting resistance of an HMA mixture is initial density. HMA mixtures that are compacted to low initial densities (typically less than 90 percent for dense-graded mixtures) are more prone to rutting than are adequately compacted mixtures. An attempt was made in this study to relate the HMA rutting performance to initial density. Figure 6-20 shows the variation of rutting as a function of the initial densities for the test track mixtures. Although there are only slight differences in the initial densities of the mixtures, higher initial densities did result in less rutting. The coarse-graded mixtures show a stronger relationship with initial density than do the fine-graded mixtures. Higher initial densities can be achieved by two methods, adding more binder and/or additional compaction. A higher amount of binder is not desired for rutting resistance as inter-particle contact can be reduced. On the other hand, adequate additional compaction can provide higher mixture stability. Since the 
measured as-constructed binder contents of the coarse-graded optimum and optimum plus one-half percent mixtures did not really differ by one-half percent (Table 6-2), the densities must have been achieved by good compaction. This may explain the improved initial density-rutting relationship for coarse-graded mixtures as compared to the finegraded mixtures.

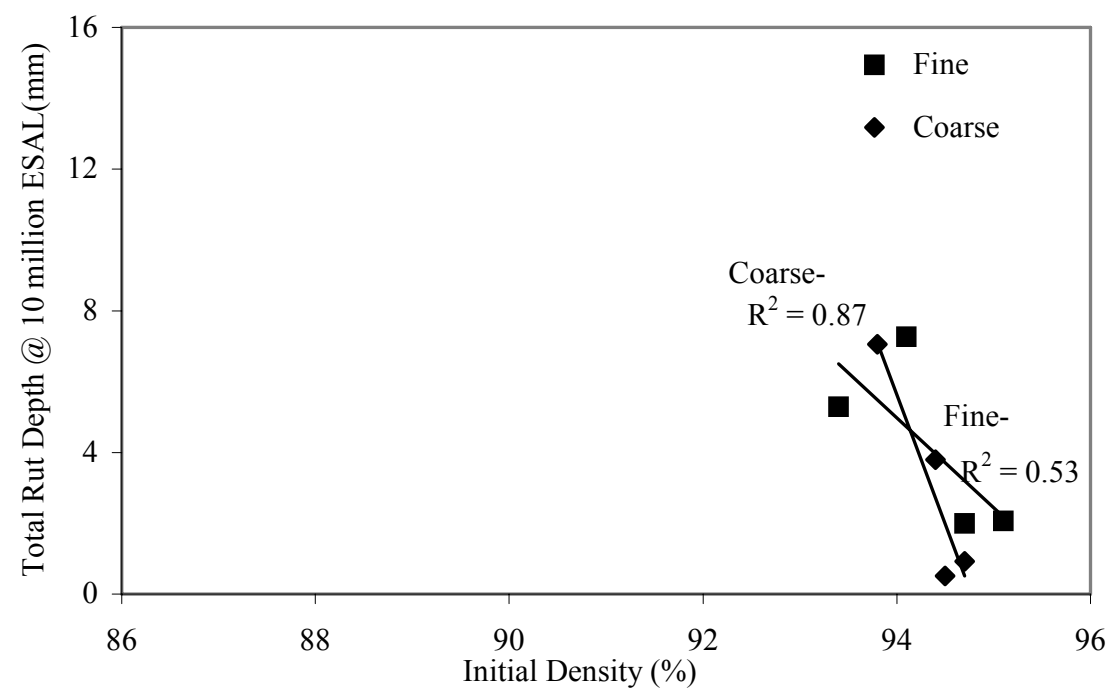

Figure 6-20 Relationship between Initial density and Rutting for the Test Track

Figure 6-21 shows the effect of initial density on the rutting performance of the mixtures tested in the APT. Incremental changes in the initial densities resulted in lower rutting potential and a moderate correlation $\left(\mathrm{R}^{2}=0.36\right)$ for the HMA mixtures tested at optimum binder contents. The mixtures tested at binder contents higher than optimum did not indicate a clear trend (very weak correlation). The initial densities of the mixtures with optimum binder content vary from 89.3 to 91.7 percent. The initial densities of the APT mixtures with one-half percent above optimum binder contents vary from 87.3 to 95.7 percent. According to the results, higher initial densities did show improved rutting 
performance when initial densities were within a normally accepted initial density range, generally recommended to be 7 to 10 percent.

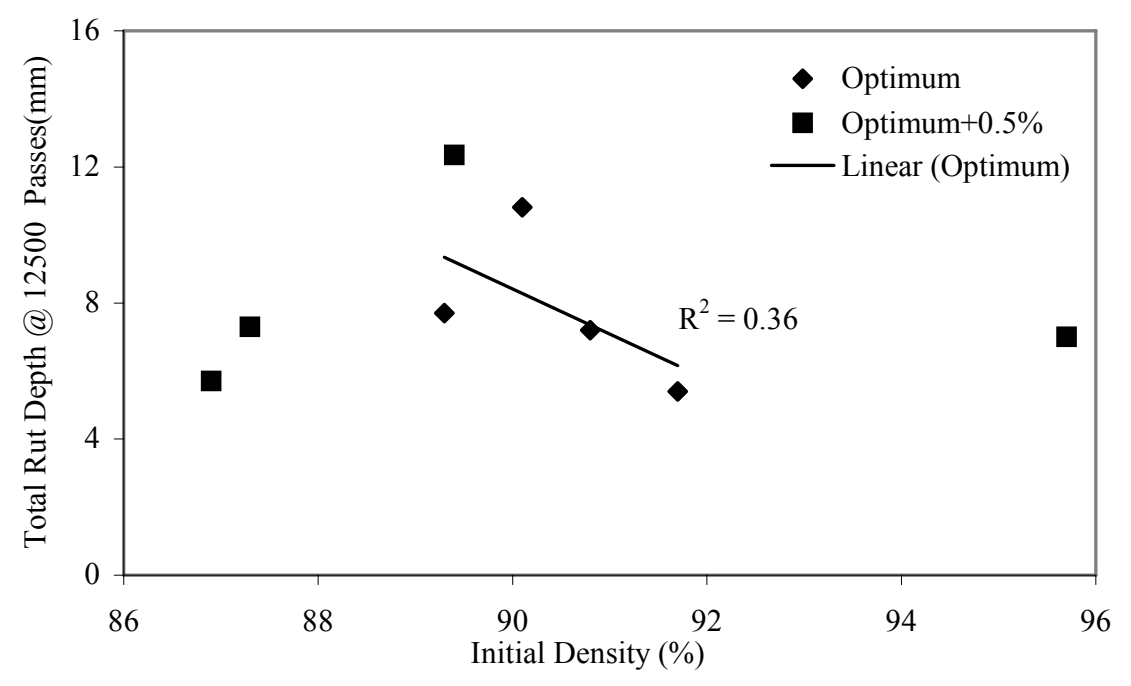

Figure 6-21 Relationship between Initial density and Rutting for the APT

Figure 6-22 shows the variation of total measured rut depth as a function of the initial densities for HMA slab specimens taken for the test track and tested in the PURWheel. Fine-graded mixtures appear to have a moderate correlation $\left(\mathrm{R}^{2}=0.43\right)$ while the coarse-graded mixture data indicate no correlation $\left(\mathrm{R}^{2}=0.07\right)$.

Figure 6-23 shows the measured rut depth as a function of initial density for slab specimens taken from the APT and tested in the PURWheel. A weak correlation $\left(\mathrm{R}^{2}\right.$ $=0.09)$ was observed for the mixtures with the optimum binder content and there appears to be no correlation for the optimum plus one-half percent mixtures. The results for the slab specimens taken from the APT and tested in the PURWheel are very similar to those 
for the APT as shown in Figure 6-21. The discussion presented for the APT rutting data would be applicable here as well.

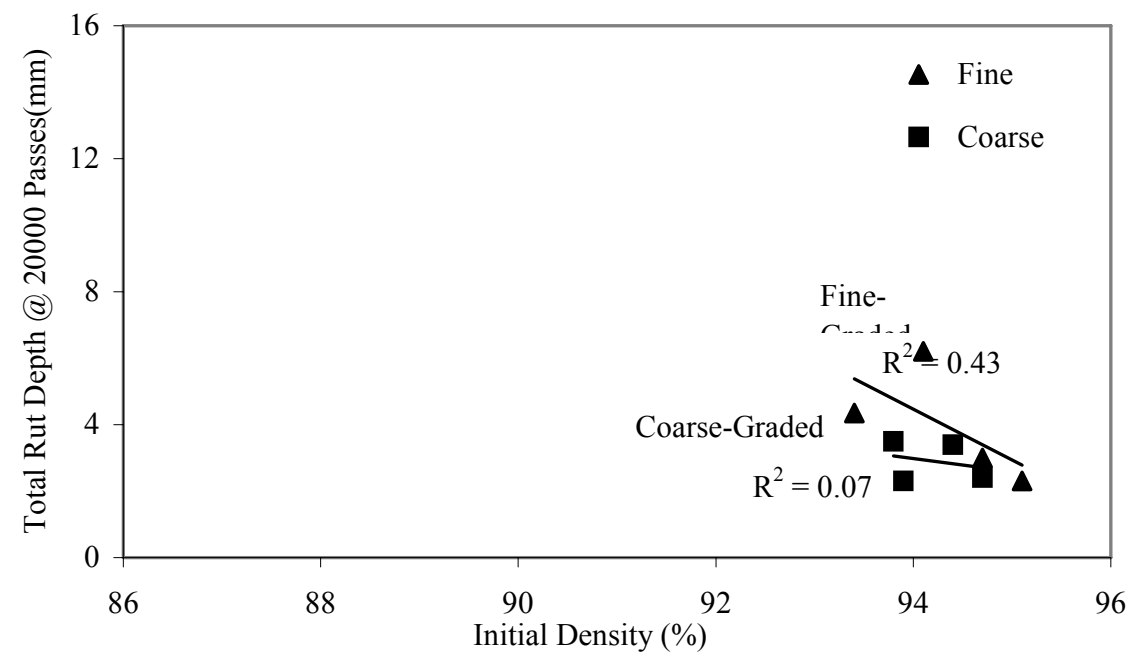

Figure 6-22 Relationship between Initial density and Rutting for the PURWheel (Track Slabs)

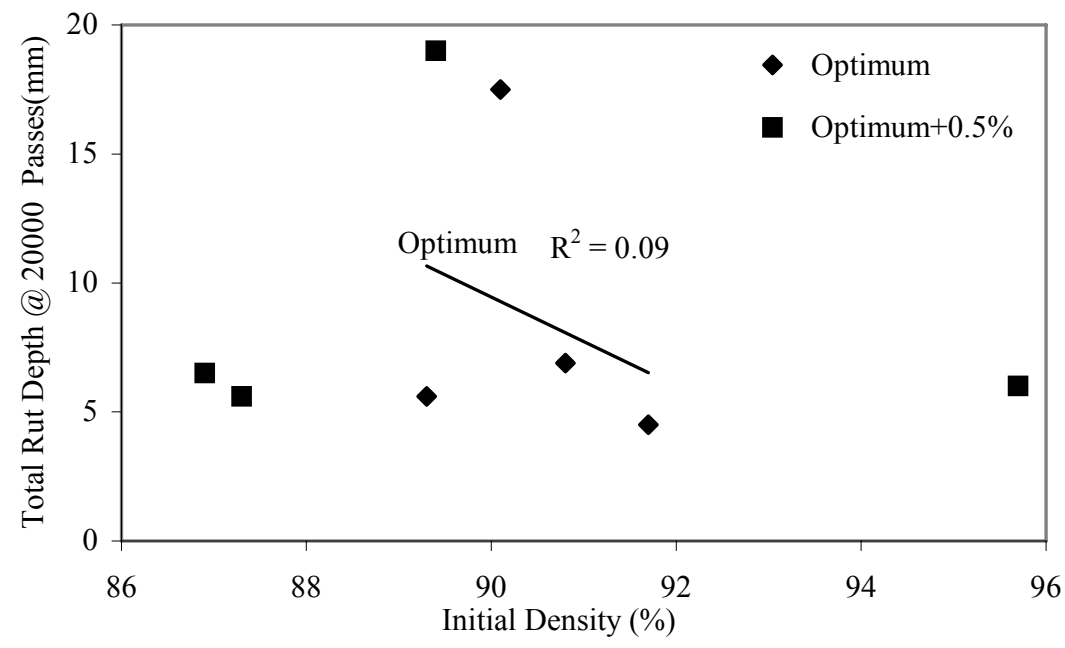

Figure 6-23 Relationship between Initial density and Rutting for the PURWheel (APT Slabs) 


\subsection{Effect of Compaction Method on Rutting Performance}

Haddock (Haddock et al., 1998) reported that PURWheel rutting performance is related to the type of compaction and mixture preparation used to fabricate the test specimens. An analysis was conducted to investigate the effect of the PURWheel linear compactor (laboratory compaction) on the rutting performance of the HMA mixtures.

Figure 6-24 shows the rutting performance relationship between laboratory compaction and field compaction (test track specimens). The data indicate a poor relationship.

Further, the rutting resistance of the laboratory-compacted slab specimens was better than that of the field-compacted slabs. It is possible that compaction in the linear compactor results in a different aggregate orientation and stronger aggregate structure. Additionally, the compaction energy between the laboratory and field methods could be quite different. This result compares well with the earlier study reported by Stiady (2000).

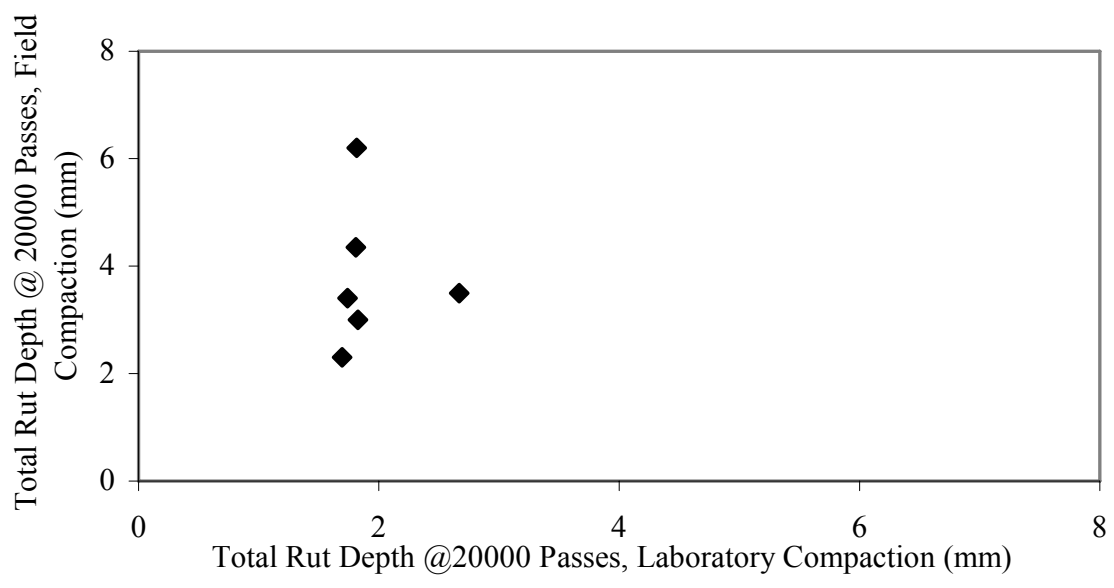

Figure 6-24 Relationship between Test Track and Linear Compaction 


\subsection{Correlation of Direct Rutting Performance}

\subsubsection{PURWheel and APT}

An attempt to directly relate the rutting performances in the APT and the PURWheel was made by comparing the APT total rut depth at 12,500 passes with the PURWheel total rut depth at 20,000 passes, as shown in Figure 6-25. The strong relationship between the APT and PURWheel agrees with a previous study conducted by Stiady (2000). This strong relationship is to be expected since the specimens tested in the PURWheel were extracted from the APT test sections prior to the traffic application and the test temperatures in both the APT and PURWheel was maintained at 50C (122F). It appears that the APT and PURWheel yield similar results when the mixtures tested in each have the same properties and are tested at the same temperatures.

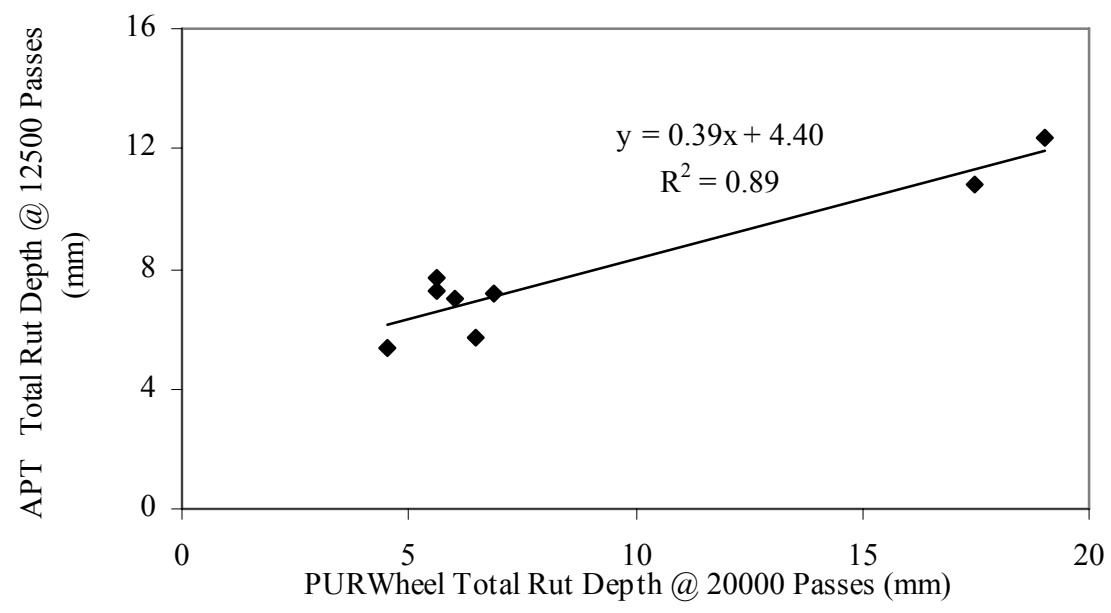

Figure 6-25 Comparison of APT and PURWheel Rutting 


\subsubsection{APT and NCAT Test Track}

The direct relationship between the APT rut depth at 12,500 passes and the test track rut depth at 10 million ESAL is plotted in Figure 6-26. The relationship appears to be fair with an observed correlation coefficient of 0.47. It is believed that this poorer correlation is due in part to differences in test temperatures and initial mixture densities. The test temperature in the APT was maintained at 50C (122F) during testing while the test track experienced daily and seasonal temperature variations. Further, a comparison of the initial densities of the APT test sections and the in-place test track sections shows that the initial densities of the test track sections were in general, higher than those in the APT with a maximum difference of $6.5 \%$ for Section N5. Variations in the temperatures and the large differences in initial in-place densities could be obscuring the actual relationship between the APT and the test track.

Figure 6-26 also shows that when the relationship between the APT and NCAT test track is grouped by binder type, the modified binder mixtures show a strong negative correlation $\left(\mathrm{R}^{2}=0.97\right)$ while the neat binder mixtures have a weak positive correlation $\left(\left(\mathrm{R}^{2}=0.13\right)\right.$. This negative correlation is again an indication of differences between the modified binder mixtures at the test track and in the APT. Had the initial densities of the modified binder test sections in the APT been similar to those of the modified binder test sections at the track, the correlation would likely have been positive. 


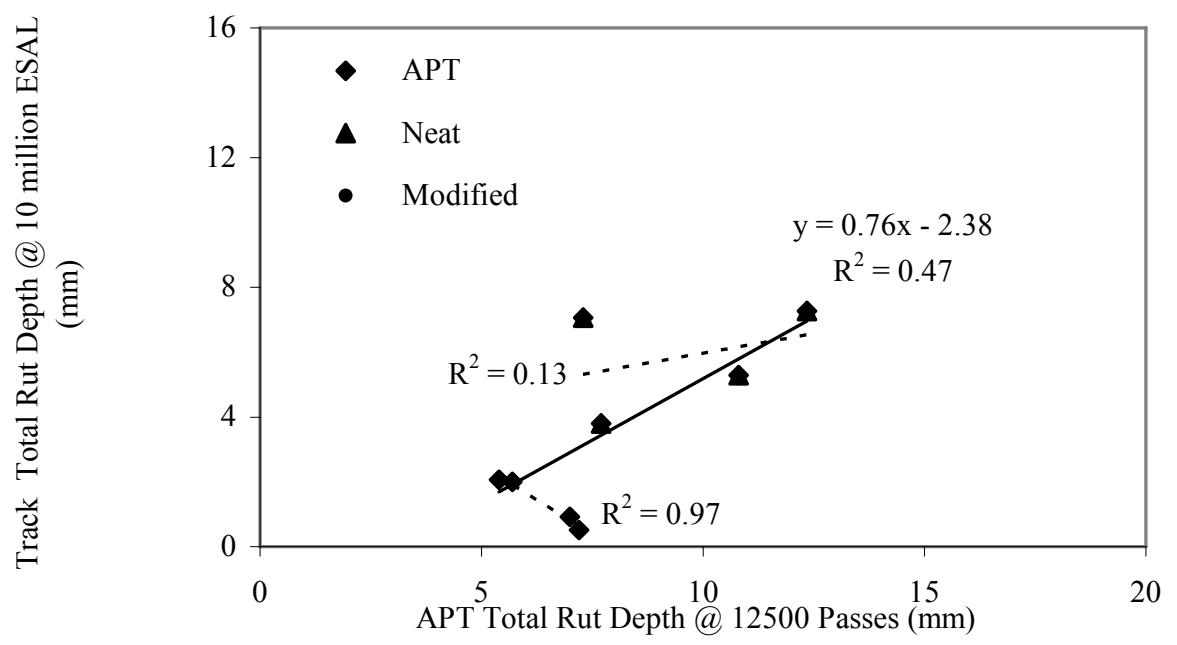

Figure 6-26 Comparison of Track and APT Rutting

\subsubsection{PURWheel and NCAT Test Track}

Figure 6-27 shows the rutting relationship between the PURWheel and the test track. The PURWheel tests were performed on the slabs taken from the APT prior to traffic application. The observed correlation between the PURWheel total rut depth at 20,000 passes and the test track total rut depth after 10 million ESAL applications is again rather weak with a correlation coefficient of 0.35 . As previously noted for the APT-Track relationship, varying temperatures coupled with the different initial densities may have reduced the strength of the relationship. Also the magnitude of the wheel load, the loading configuration, the wheel velocity, the test specimen geometry, and the boundary conditions are different for the PURWheel compared to the test track. These variables certainly must contribute to the weak correlation. Also, the correlation between the test track and APT was stronger than that of the test track and PURWheel indicating 
that the APT loading is more representative of the actual traffic than is the PURWheel loading.

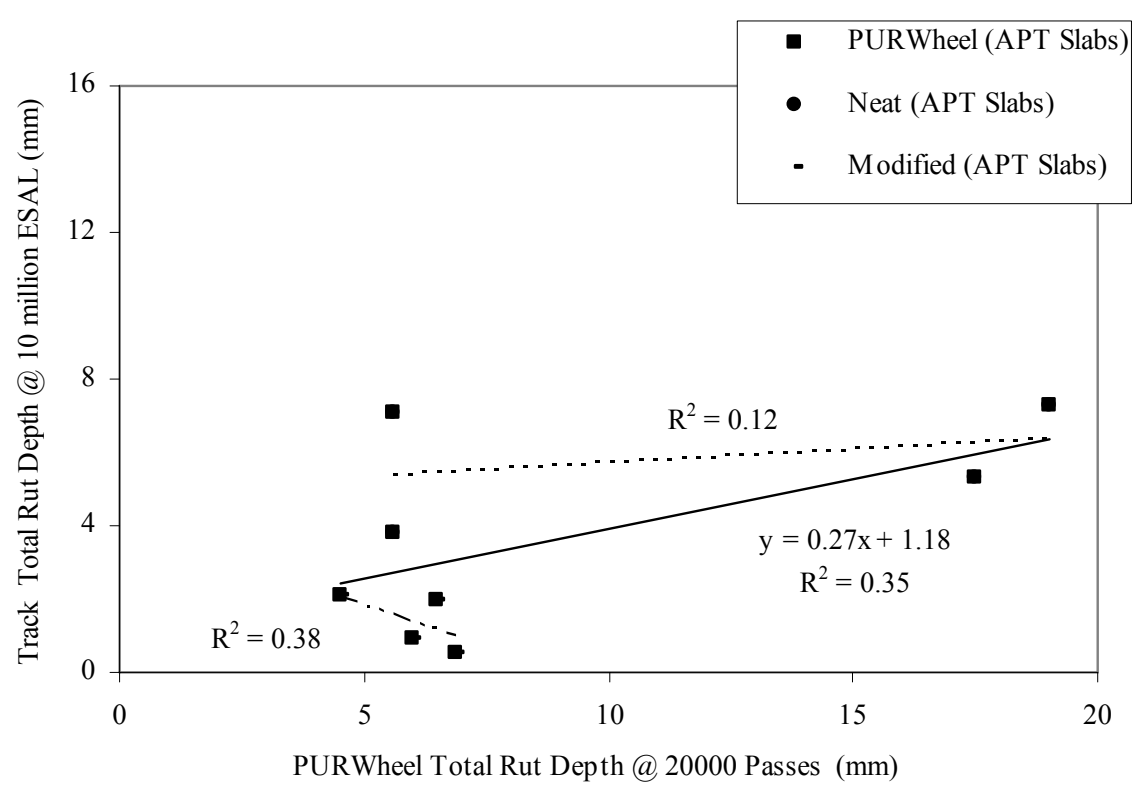

Figure 6-27 Comparison of Track and PURWheel Rutting

Figure 6-28 shows a relationship between the PURWheel rut depth at 20,000 passes and the test track rut depth after 10 million ESAL. This data is for PURWheel slab specimens that were cut from the inside lane of the test track and therefore never received any traffic. In this case, a relatively strong correlation exists with an $\mathrm{R}^{2}$ value of 0.69 . These slab specimens had initial densities similar to the initial in-place densities of the test track sections and were compacted in the same manner. The unexplained variation in the correlation is most likely related to the varying seasonal temperature conditions of the track and the difference in the test conditions as previously discussed. It also shows that 
when the data is grouped among binder types, both modified binders and neat binders showed very weak (positive) correlations with $\mathrm{R}^{2}$ values of 0.11 , and 0.31 respectively.

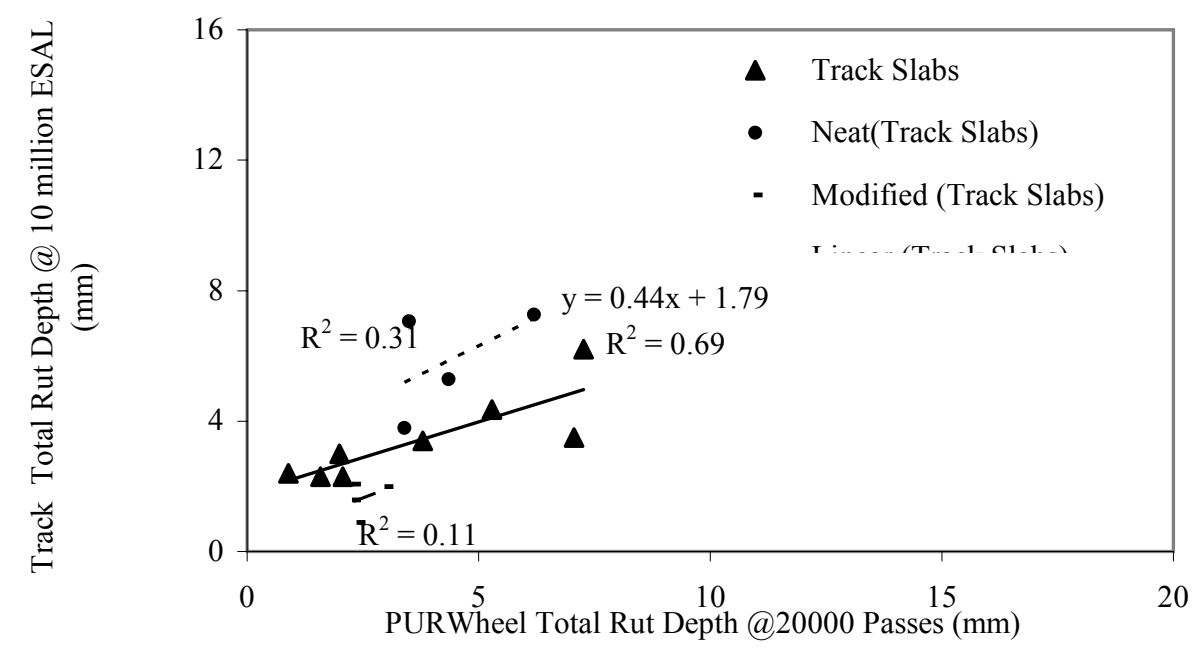

Figure 6-28 Comparison of Track and PURWheel Rutting

It is important to note the significant improvement in the correlation between rutting in the PURWheel and at the test track when the PURWheel specimens were taken from the test track rather than APT. Realizing that the main difference between these two sets of specimens is the initial in-place densities, the existence of a good correlation between the PURWheel and test track most likely does exist provided that the field HMA mixture properties and compaction are duplicated when preparing PURWheel specimens.

\subsubsection{Dynamic Modulus Test and NCAT Track}

Table 6-5 shows the dynamic modulus measured at $10 \mathrm{~Hz}$ for both test track (tested at 64C (148F)) and APT samples (tested at 40C (104F)). The total rut depth at the test track after 10 million ESAL is plotted as a function of the dynamic modulus of test 
track and APT samples as shown in Figure 6-29 and Figure 6-30, respectively. There is very little variation in the dynamic modulus results from the test track mixtures and there does not appear to be any correlation.

Table 6-5 Dynamic Modulus Test Data

\begin{tabular}{|c|c|c|}
\hline \multirow[b]{2}{*}{ Section } & \multicolumn{2}{|c|}{ Dynamic Modulus@10 Hz (x 106 psi) } \\
\hline & $\begin{array}{c}\text { Track Samples } \\
(64 \mathrm{C})\end{array}$ & APT Samples (40C) \\
\hline N1 & 0.196 & 0.183 \\
\hline $\mathrm{N} 2$ & 0.167 & 0.213 \\
\hline N3 & 0.211 & 0.196 \\
\hline N4 & 0.202 & 0.167 \\
\hline N5 & 0.251 & 0.158 \\
\hline N6 & 0.196 & 0.232 \\
\hline N7 & 0.279 & -1 \\
\hline N8 & 0.262 & -1 \\
\hline N9 & 0.310 & 0.451 \\
\hline N10 & 0.175 & 0.226 \\
\hline
\end{tabular}

${ }^{1}$ These sections were not tested in the APT.

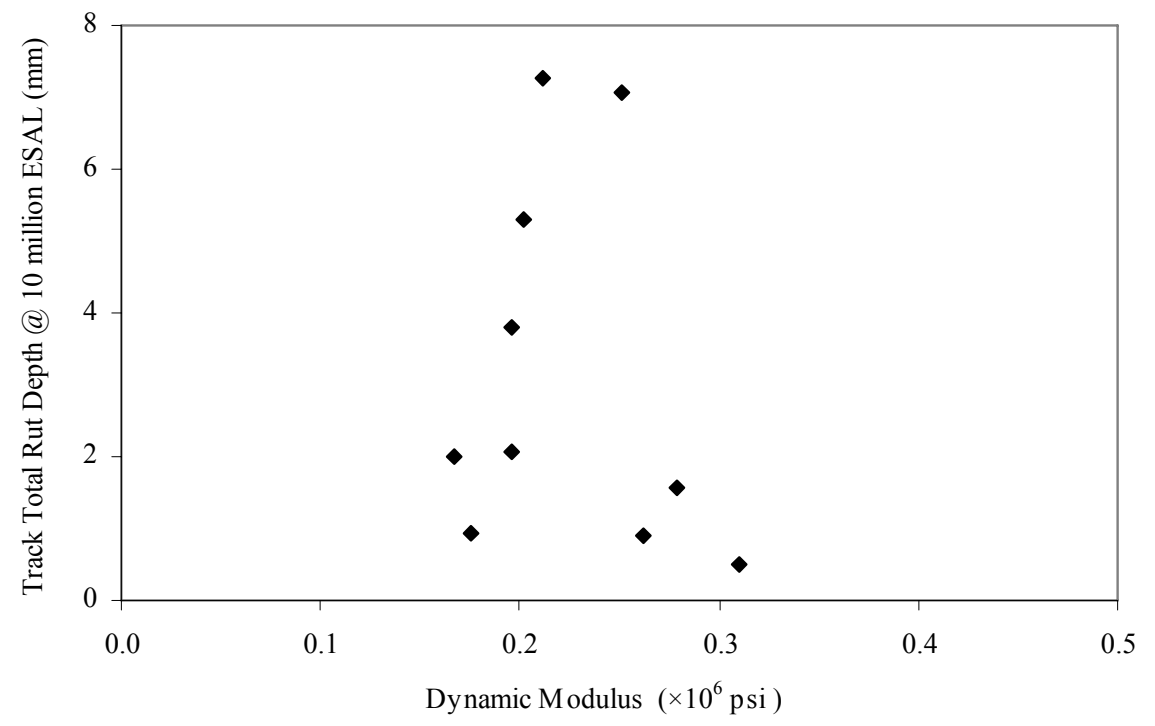

Figure 6-29 Relationship between Dynamic Modulus and Track Rut Depth (Track Samples) 


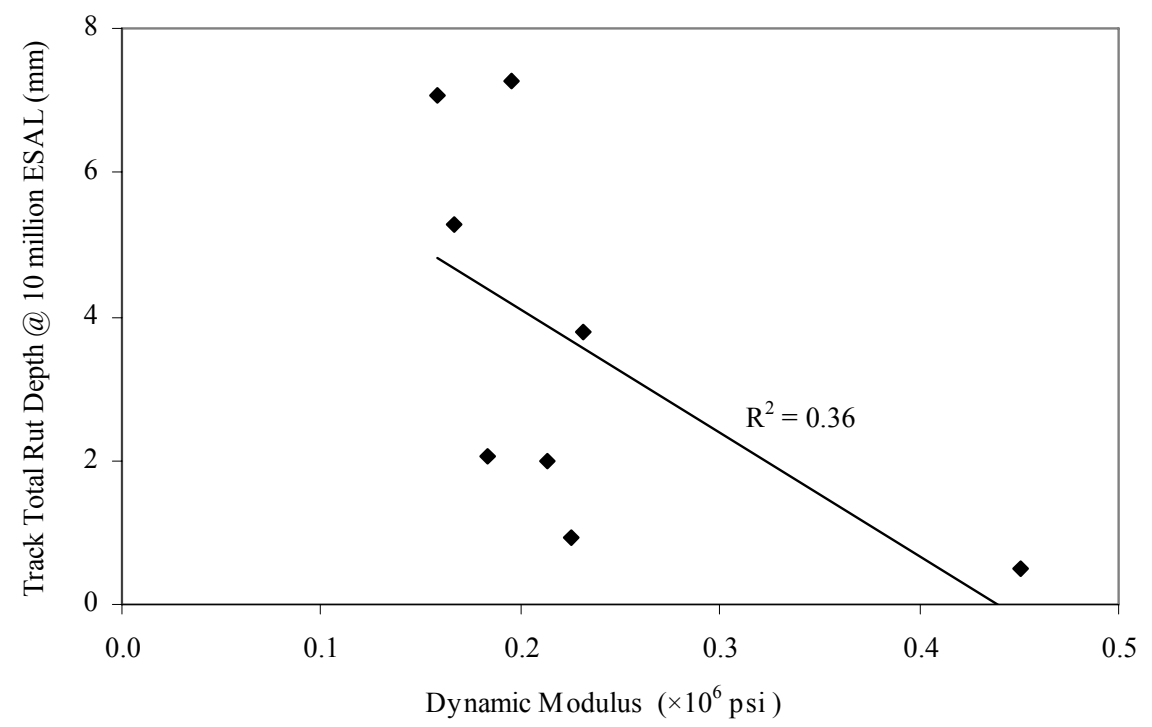

Figure 6-30 Relationship between Dynamic Modulus and Track Rut Depth (APT Samples)

For the results produced using mixtures taken during APT construction, there does appear to be a weak correlation $\left(\mathrm{R}^{2}=0.36\right)$ between the rut depths at the test track and the dynamic modulus results. However, it should be noted that this weak correlation is due to one mixture that shows a much higher dynamic modulus. If this data point were removed, there would again be no correlation between test track rut depths and dynamic modulus results. 


\section{CHAPTER 7 FINITE ELEMENT MODELING}

\section{$\underline{7.1 \text { Overview }}$}

Finite element modeling (FEM) has been used to solve a variety of problems in engineering, mathematics and physics. Typically, this approach is used when obtaining analytical solutions would be difficult or impossible due to the complex nature of the problem. Using the FEM approach can yield approximate solutions or even exact solutions in some cases.

In the FEM technique, the system in question is divided into small units (elements), which are linked through nodes. Individual element properties are then combined to establish global equations. Loads and boundary conditions are defined and a set of unknowns is determined. FEM methods provide many benefits including (Logan, 1993):

1. Applying dynamic loading conditions;

2. Model irregular geometry;

3. Define an infinite number of boundary conditions;

4. Account for non-linear materials;

5. Modify and edit an existing model conveniently; and

6. Apply a variable element size. 


\subsection{Application of Finite Element Modeling}

One of the objectives of this study is to establish a rutting performance relationship between the APT, the PURWheel, and in-service (track) pavement. Rutting is dependent on many test variables, such as wheel load, wheel velocity, pavement geometry, tire contact pressure, boundary conditions, and temperature (Hua, 2000). As described in Chapters 4 and 5, these variables are different for the APT, the PURWheel, and in-service pavements. Therefore, a direct rutting performance relationship between the devices would not fully explain the material performance. For example, the PURWheel velocity is $0.02 \mathrm{kph}(0.012 \mathrm{mph})$ whereas the velocity of the APT carriage is $8 \mathrm{kph}$ (5 mph). Consequently, the rutting performance of a given HMA mixture in the APT and the PURWheel are different. These differences are even greater when one includes track data.

It is also possible that the rut development of two HMA mixtures could be different at a different number of wheel passes. For example, HMA mixture A could have better performance at 5,000 wheel passes compared to HMA mixture B. However, at 10,000 wheel passes, HMA mixture B might have better performance than mixture A. This example illustrates the drawback of simply comparing total rut depth at a given number of wheel passes to evaluate the rutting performances of HMA mixtures.

One way to eliminate the problems of comparing rutting performance of various mixtures tested in different devices is to use fundamental material properties that are independent of the test conditions and test devices (Hua, 2000). Additionally, the material properties estimated from one device can be used as input to predict the rutting performance in another device. 
FEM is capable of capturing the rutting of HMA mixtures (Zaghloul and White, 1993) (Huang, 1995) (Hassan, 1996) (Pan, 1997) (Hua, 2000) (Fang, 2000). Each test variable can be carefully incorporated into the modeling process so that the effect of the test variables on rutting performance is accounted for and understood without performing actual physical testing. For example, HMA material properties can be used to compare the rutting performances of different HMA mixtures when tested in a single device (Hua, 2000). Further, the material properties can be compared for a given mixture, tested in more than one device, instead of the total rut depth, in order to establish rutting performance relationships. The necessary material properties are calculated using the rut development history obtained from performance tests and the predicted rut development from FEM. Further, it is possible to predict rutting performance at different test conditions using the calculated material properties for a given HMA mixture. The significance of each test variable on rutting performance can then be evaluated and used in future experimental designs and improvements to the test devices. A better understanding of HMA pavement rutting can ultimately be achieved.

A general-purpose finite element program known as ABAQUS was used in this study based on its successful application in previous research studies (Zaghloul and White, 1993) (Huang, 1995) (Hassan, 1996) (Pan, 1997) (Hua, 2000) (Fang, 2000). Typically, input files for the analyses are created using the ABAQUS interface ABAQUS/CAE. This interface allows the user to conveniently input appropriate model geometry, material properties, loading and boundary conditions, and mesh and analysis procedures through different modules. 


\section{$\underline{7.3 \text { ABAQUS/CAE Interface }}$}

ABAQUS software is integrated with the ABAQUS/CAE interface to define the various components required to complete a FEM analysis. The interface consists of several modules, eight of which are described here (ABAQUS/CAE, 2001).

1. Part Module: Used to define and generate the individual parts needed for the model geometry. Three types of parts, deformable, analytical rigid, and discrete rigid are available and are built on a base feature. Modifications of the base feature would result in the required geometry of the part. The base feature is defined using shape and type. The shape represents the topology of the feature, i.e., solid, shell, or wire. The type refers to the method that would be used to produce a part. Four methods are available in the ABAQUS/CAE, planar, extrusion, revolution, and sweep.

2. Property Module: This module is used to define materials, sections, and section assignments to the parts generated in the part module. A wide range of material types including plastic and visco-pastic can be defined using the material option. Many types of sections can be defined, including homogeneous solid sections, generalized plain strain sections, and beam sections.

3. Assembly Module: Parts are created with respect to a local coordinate system. Using the assembly module, different parts are positioned to create an assembly of the model in a global coordinate system. Positioning of the parts is performed using constraints, or rotations and translations. 
4. Step Module: This is used to define analysis types, output requirements, and contact controls. Different analysis procedures are available for different material types. For the creep material model, the VISCO procedure was used. This procedure requires the total analysis time, the maximum and minimum time increments, and an error tolerance called CETOL. In general, automatic time increments are preferred when CETOL is defined. This parameter is estimated using the following expression:

$$
C E T O L=\frac{\sigma_{e r r}}{E}
$$

where,

$$
\begin{aligned}
& \sigma_{\text {err }}=\text { acceptable level of stress error; and } \\
& \mathrm{E}=\text { typical elastic modulus. }
\end{aligned}
$$

A value of $10^{-4}$ was used for CETOL in the previous studies (Hua, 2000) (Fang, 2000) as well as this study. This value is based on an $\sigma_{\text {err }}$ of $69 \mathrm{kPa}(10$ psi) and an $\mathrm{E}$ on the order of $690,000 \mathrm{kPa}(100,000 \mathrm{psi})$.

5. Interaction Module: Provided to create the thermal and mechanical interaction between the zones, or regions of a model. Further, it is used to define interactions between the zones and/or regions of the model and the surrounding environment. In the APT and the test track modeling, elastic foundation boundary conditions for the longitudinal pavement edge were defined using the interaction module. An elastic foundation is defined by assigning foundation stiffness.

6. Load Module: Defines the loads, fields, and boundary conditions of the model. Its magnitude, area of application, and time of application define a 
load. The amplitude option helps the user to generate time variation of the load. Appropriate boundary conditions should be applied for realistic modeling of a given problem.

7. Mesh Module: This module is used to generate the appropriate mesh for the assembly of the model. Element types and sizes are defined and applied for the mesh generation to a region of the model or the whole assembly.

8. Job Module: This provides the opportunity to perform an analysis, monitor the progress of the analysis, and if required, discontinue a job while it is in process. The job type includes complete analysis, restart, recover, data check, and continue analysis.

\subsubsection{Quasi-Static Analysis}

In the ABAQUS software, problems involving time independent static loading behavior are analyzed by static analysis, where as time-dependent, static loading behavior such as creep, viscoelasticity, and visco-plasticity are analyzed by quasi-static analysis (ABAQUS/Standard Users' Manual, 2001). Quasi-static analysis can be linear or nonlinear and is applicable when inertia effects (mass) are negligible. The VISCO procedure is called to perform the analysis by selecting that option in the step module or by using the keyword in the input file. Usually, this type of analysis represents a loading application for a longer period of time. 

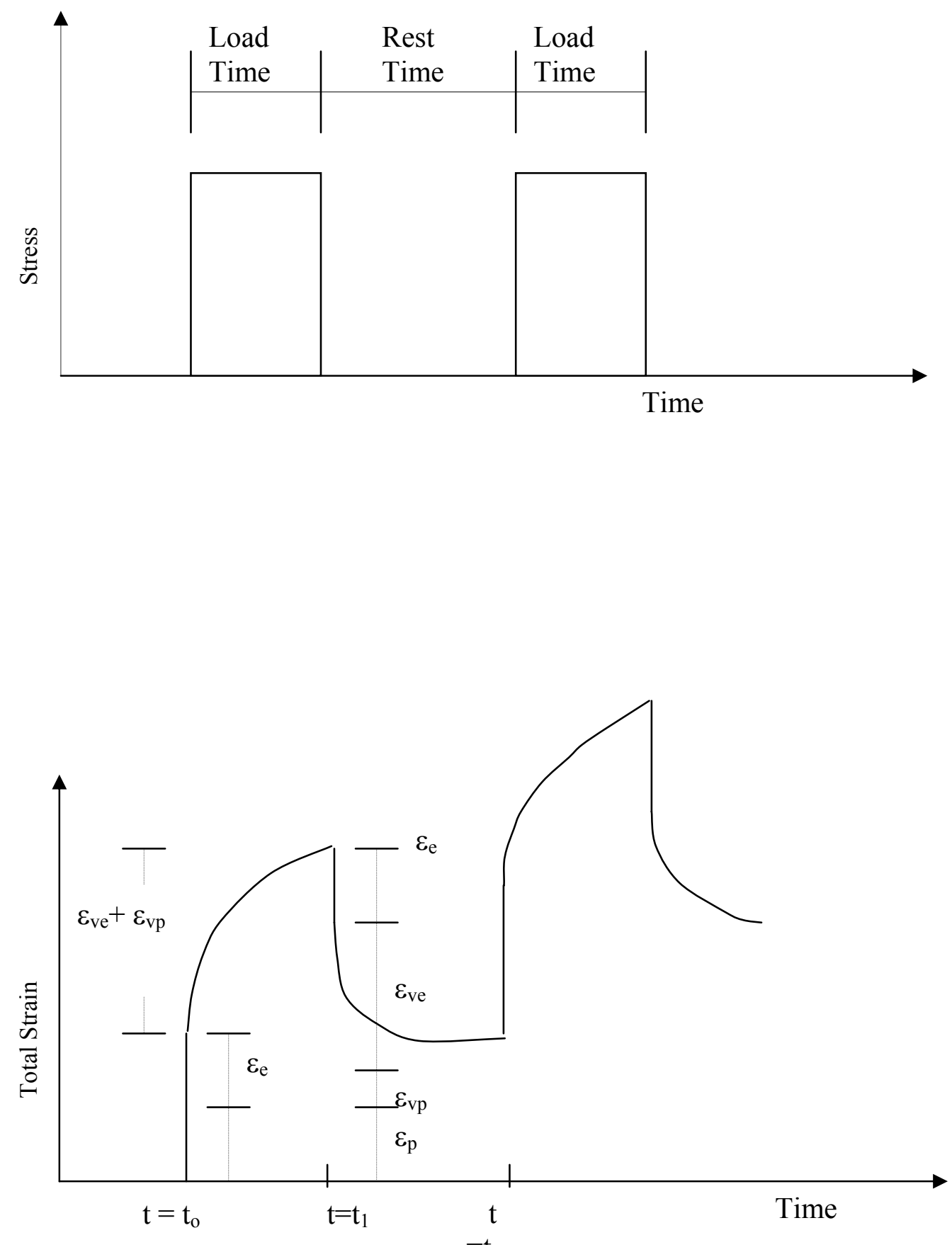

Figure 7-1 Schematic Representation of the Stress and Strain Components (After Pirabarooban, 2002) 


\subsection{Creep Models}

When subjected to repeated loads, cylindrical HMA specimens show elastic $\left(\varepsilon_{\mathrm{e}}\right)$, plastic $\left(\varepsilon_{\mathrm{p}}\right)$, visco-elastic $\left(\varepsilon_{\mathrm{ve}}\right)$ and visco-plastic $\left(\left(\varepsilon_{\mathrm{vp}}\right)\right.$ responses (Perl, et al., 1983) as shown in Figure 7-1. With reference to Figure 7-1, when a load is applied, the total strain at time $t$ equals $t_{0}$ can include elastic and plastic strains depending on whether or not the applied stress exceeds the yield strength of the material or if the stress occurs instantaneously. The stress remains constant for times $t_{0}<t<t_{1}$ and visco-elastic and visco-pastic strains start to develop. During the unloading at $t$ equals $t_{1}$, the elastic strain is recovered. For times $t_{1}<t<t_{2}$, the visco-elastic strain is recovered. Both plastic and visco-plastic strains are irrecoverable and contribute to the permanent deformation observed at time $t_{2}$ (end of the first loading cycle). Plastic stain develops instantaneously while visco-plastic strain develops at a constant stress level with time. A creep material model can characterize this time dependent nature of HMA.

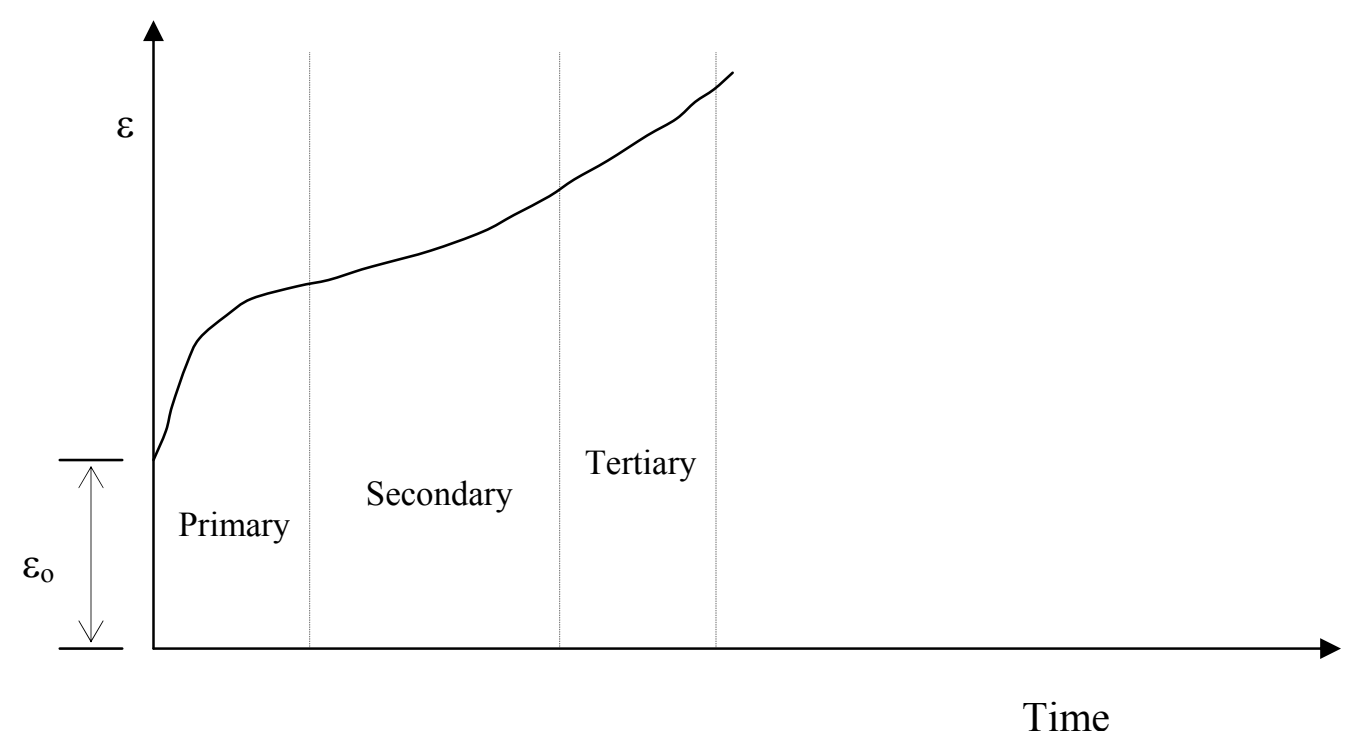

Figure 7-2 Typical Creep Curve (After Pirabarooban, 2002) 
A typical creep curve is presented in Figure 7-2. As illustrated in the figure, there are three regions; primary, secondary and tertiary (Kraus, 1980). The strain at time t equals zero, $\varepsilon_{0}$, could include elastic and/or elasto-plastic responses depending on the applied stress level and the yield strength of the material. This response occurs instantaneously. Generally, as time increases, the creep strain also increases and the material fails (rupture) after exhibiting some deformation in the tertiary region. However, not all materials exhibit all of the three regions. Some do not develop the secondary region while others do not develop the tertiary region.

Within the secondary region, the variation between creep strain and time is linear and can be described as:

$$
\varepsilon_{\mathrm{C}}=\mathrm{f}(\sigma, \mathrm{T}, \mathrm{t})
$$

where,

$$
\begin{aligned}
& \varepsilon_{\mathrm{C}}=\text { creep strain; } \\
& \sigma=\text { stress }(\mathrm{psi}) ; \\
& \mathrm{T}=\text { temperature }(\mathrm{C}) ; \text { and } \\
& \mathrm{t}=\text { time }(\mathrm{s}) .
\end{aligned}
$$

Two different techniques can be used to derive a mathematical formula that relates creep stain to time, temperature, and stress (Kraus, 1980). The first technique assumes that the material behavior depends on the current state while the second considers material behavior to depend on past events. Therefore, the first technique results in the derivation of an equation of state while the second deals with memory theory. The first technique was used to derive the theoretical expressions in this study 
because of prior application and experience, the need for minimal test data, and simplicity.

Using the equation of state method, the relationship between creep strain, stress, temperature, and time can be expressed in the form of Equation 7.3.

$$
\varepsilon_{c}=\frac{A}{m+1} \sigma^{n} t^{m+1}
$$

In this equation, $\mathrm{A}, \mathrm{m}$, and $\mathrm{n}$ are constants that are functions of the temperature. This relationship is known as the Bailey-Norton law and is derived for primary and secondary stages of creep. The constant $\mathrm{m}$ takes values ranging from -1 to 1 and $\mathrm{n}$ is a fraction.

Differentiating equation 7.3 with respect to time yields Equation 7.4, where $\varepsilon_{\mathrm{c}}$ is the creep strain rate and the other variables are as previously defined.

$$
\dot{\varepsilon}_{c}=A \sigma^{n} t^{m}
$$

This equation establishes that the creep strain rate is a function of stress, time and temperature (inclusion of material constants) and is known as the time-hardening formulation. Note the exclusion of the stress variation with time in the derivation process. This is a drawback to the equation of state method. The time-hardening formulation is only applicable for the conditions where changes in stress occur over a long period of time.

Substitution of time in Equation 7.4 results in the strain-hardening formulation. Equation 7.3 can be rearranged to yield Equation 7.5.

$$
t=\left(\frac{\varepsilon_{c}}{(m+1) A \sigma^{n}}\right)^{\frac{1}{m+1}}
$$


Substituting Equation 7.5 into Equation 7.4 yields Equation 7.6, the strain-hardening formulation.

$$
\dot{\varepsilon}_{c}=\left(A \sigma^{n}\left[(m+1) \varepsilon_{c}\right]^{m}\right)^{\frac{1}{m+1}}
$$

where,

$$
\begin{aligned}
& \varepsilon_{\mathrm{c}}=\text { uniaxial equivalent creep strain rate; } \\
& \sigma=\text { uniaxial equivalent deviator stress (psi); } \\
& \varepsilon_{\mathrm{c}}=\text { equivalent creep strain; and } \\
& \mathrm{A}, \mathrm{m}, \mathrm{n}=\text { material constants. }
\end{aligned}
$$

For this formulation, the creep strain rate is related to the stress, creep strain, and temperature (inclusion of material constants). The strain-hardening formulation is considered more accurate than the time-hardening equation and is used for obtaining numerical solutions using computers (Kraus, 1980). However, the time-hardening formulation is simpler to solve. Therefore, the time-hardening formulation is preferred for obtaining analytical solutions. The time-hardening form of the power law is most appropriate for application with a constant stress condition while the strain-hardening is applicable for variable stress conditions. The magnitude of the stress levels should be relatively low for both cases. Due to the reasons cited, the strain-hardening power law was selected and used in the FEM process.

If the conditions of creep and plasticity exist simultaneously, the finite element approximation may generate large errors (ABAQUS/Standard Users Manual, 2001). This situation arises when a load increment is applied over a long-term period. However, short-term loading, which creates plastic yielding followed by creep, does not generate 
large errors. The finite element modeling process of this study used short-term load increments and solutions can be considered to be reasonably accurate. Solutions within 10 percent are considered to be accurate.

\subsection{Loading Methods}

Simulating an actual APT loading sequence can be a difficult task in finite element modeling. It requires the loads to be applied from one end of the pavement test section to the other varying with time over a different set of elements that are in the load path. This procedure must be repeated until the required number of load passes is applied. This explicit load simulation procedure requires a long computational time, since there are many steps. Alternatively, a simplified loading method was introduced and used by Huang (1995) and subsequently by Pan (1997), Hua (2000), and Fang (2000). With this simple approach, the load is first applied over the entire length of the wheel path. The total cumulative loading time is then estimated using the number of wheel passes and the time required for traversing the tire print of the wheel during a single pass. The time for a single pass is estimated based on the tire print length and the wheel velocity. This simpler approach drastically reduces the computational time the load is applied by using a single step.

For illustration purposes, in Figure 7-3, a pavement section is modeled using a coarse mesh consisting of only four element rows. The conversion of multiple-step loadings (four element rows, each loaded to time $\mathrm{T}$, in a total of four steps) to a singlestep loading (all element rows loaded to time $\mathrm{T}$, in one step) applied along the entire wheel path in the longitudinal direction is shown. Note that for a single pass, using 
individual mesh element loading, the number of loading steps is determined by the number of element rows (n) in the longitudinal direction. Therefore, for $\mathrm{N}$ wheel passes a total of $\mathrm{N} \times \mathrm{n}$ loading steps would be required. To apply 10,000 wheel passes to the mesh in Figure 7-3 would therefore require 40,000 load steps $(10,000 \times 4)$. However, using the simplified loading method, only a single loading step is needed. Since the computational time is greatly reduced by using the simplified loading method, a decision was made to use it. If the wheel load duration and magnitude is known using a multiple-step loading method, one could use a single-step load of that duration and magnitude to determine the effects of the loading.

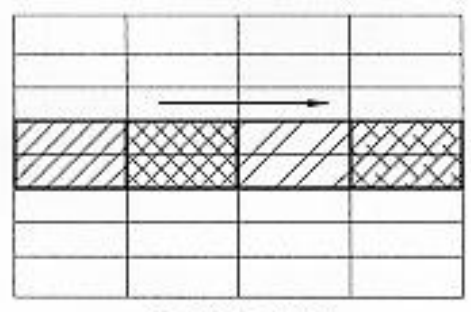

Loading Area

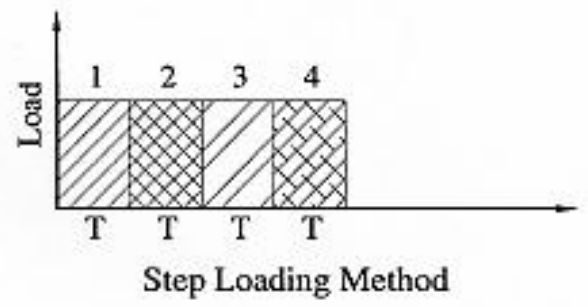

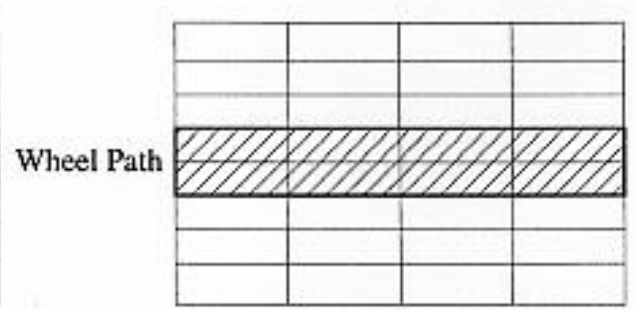

Loading Area

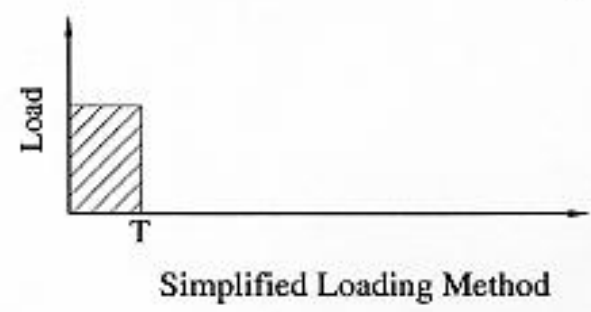

Figure 7-3 Simplified Loading Method (After Hua, 2000)

PURWheel loading consists of back and forth wheel movements over the test slab as described in Chapter 5. Since concrete blocks are used in the sample box to help secure the test specimen, in the forward movement the wheel first travels over the concrete blocks, the test slab, and the concrete blocks on the far end of the box. Then, it 
reverses its movement. It is important to note that this type of loading sequence causes a time interval during which the slab is not loaded. While it is possible that some sample rebounding could occur during this unloading, the PURWheel passes are applied within a short period of time and the relaxation time is therefore small compared to the total loading time. It is therefore reasonable to ignore the relaxation effects (Hua, 2000). It can be assumed that the slab is continuously loaded with the total loading time determined by the number of wheel passes and the time required for traversing the tire print of a single wheel pass. PURWheel loading in the FEM was achieved as described above.

A previous study by Hua (2000) confirmed the validity of the simplified loading. He proved that the total rutting predicted using the simplified loading was equivalent to the multiple load method for the PURWheel. He also proved that the loading time is not dependent on the element size in the finite element mesh.

\subsection{Test Track Modeling}

\subsubsection{Test Track Geometry}

As with the APT, a two-dimensional finite element model was used to model the NCAT test track pavement structure and loading. Figure 7-4 shows the details of the typical pavement cross-section on the test track. It consisted of a 100-mm (4-in.) thick HMA surface course, a 375-mm (15-in.) intermediate course, a 125-mm (5-in.) black base course, a 150-mm (6-in.) open graded aggregate base, and a 300-mm (12-in.) improved subgrade. The pavement cross section was identical for every test section. However, the type of HMA surface mixture varied for each section. 


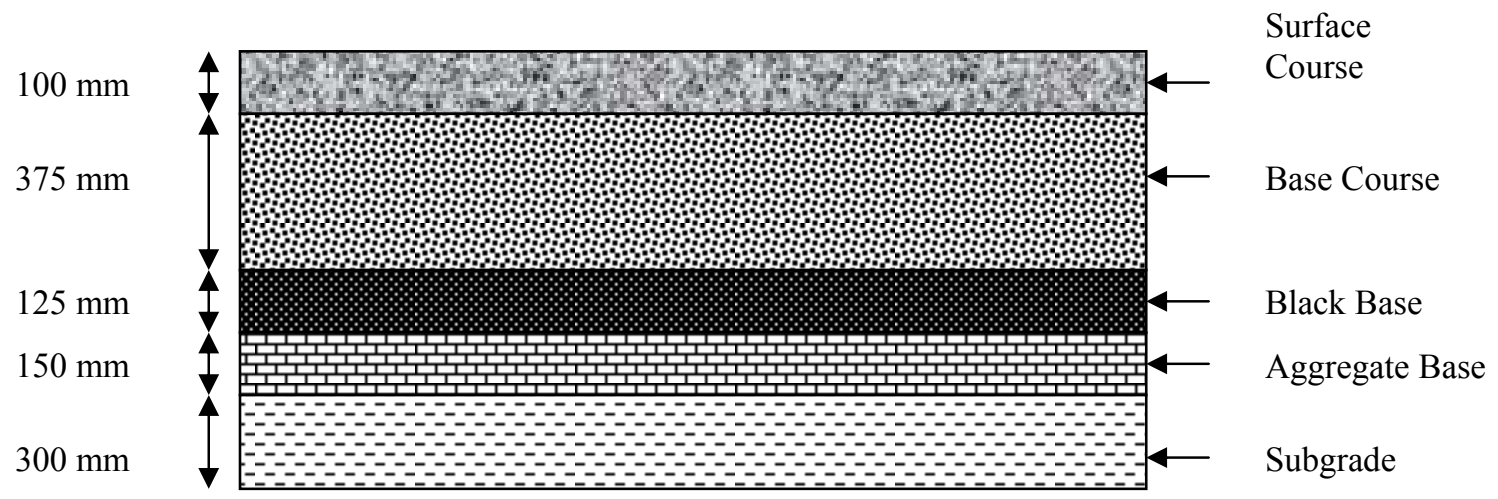

Figure 7-4 NCAT Test Track Cross Section

\subsubsection{Element Type}

Plain strain conditions were assumed in the finite element modeling of the test track. Consequently, a four-node linear plain strain element (CPE4) was selected from the ABAQUS standard library of elements. The node and face ordering is shown in Figure 75.

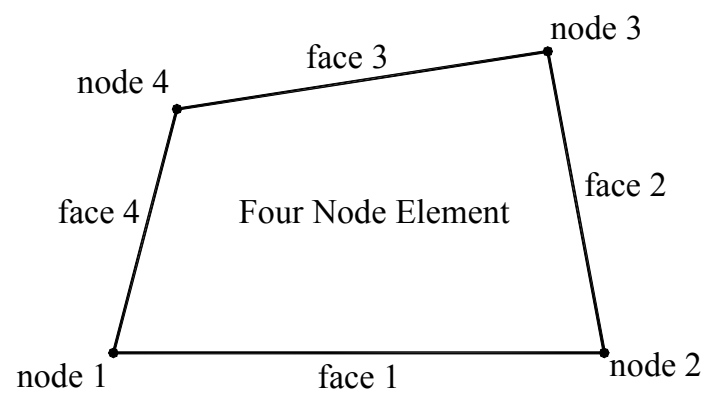

Figure 7-5 Four-node Bilinear Plane Strain Element (CPE4)

\subsubsection{Boundary Conditions}

Realizing that the stresses induced at the subgrade are low and that the pavement was structurally designed to accommodate deformations in the surface layer only, a fixed 
boundary condition was assigned to the bottom of the pavement structure, i.e., the bottom of the subgrade was assumed to be fixed. Surface layer was represented using a creep model, while other layers were represented using a linear elastic model. Representative Young's modulus and Poisson's ratio values were assigned to each layer in the pavement structure. Figure 7-6 depicts the boundary conditions used in the test track modeling.

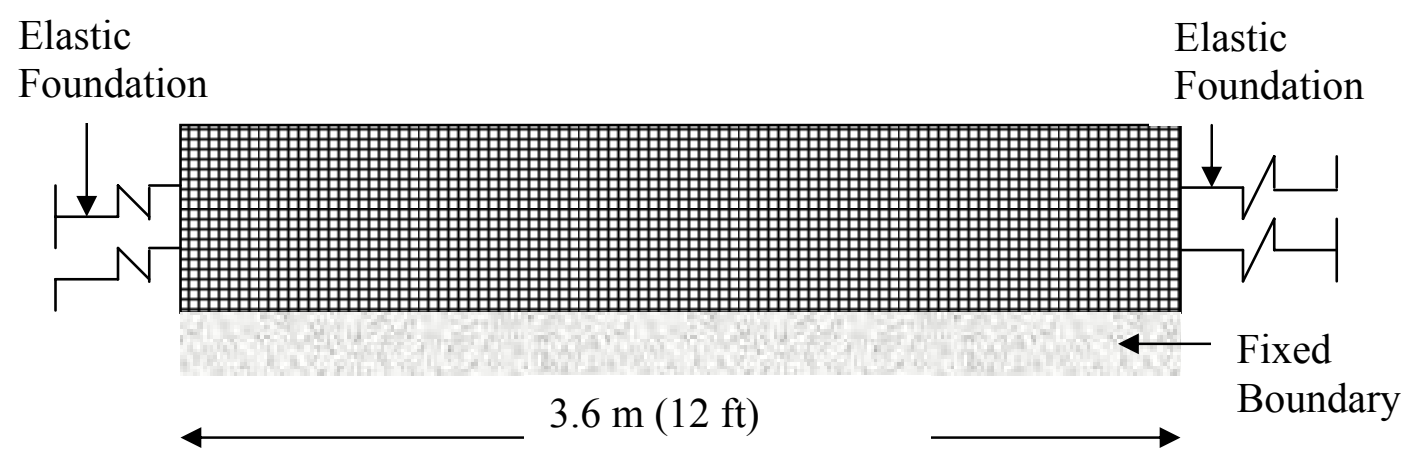

Figure 7-6 NCAT Test Track Boundary Conditions

The actual test lanes at the track were surrounded by paved shoulders on the outside and by a second travel lane on the inside. The lateral movements were resisted by the passive action of the adjoining HMA mixtures. Subsequently, elastic foundation boundary conditions were assigned to the sides of the pavement structure. A sensitivity study was conducted first to determine the effect of elastic foundation stiffness on rutting. Figure 7-7 shows the total rut depth as a function of the foundation stiffness. As shown, the effect of the elastic foundation stiffness on rut depth is negligible and therefore a value of $75.8 \mathrm{kPa} / \mathrm{m}(500 \mathrm{pcf})$ was used in the model. Since wheel wander was incorporated into the modeling, no axis symmetry boundary conditions were applicable. 


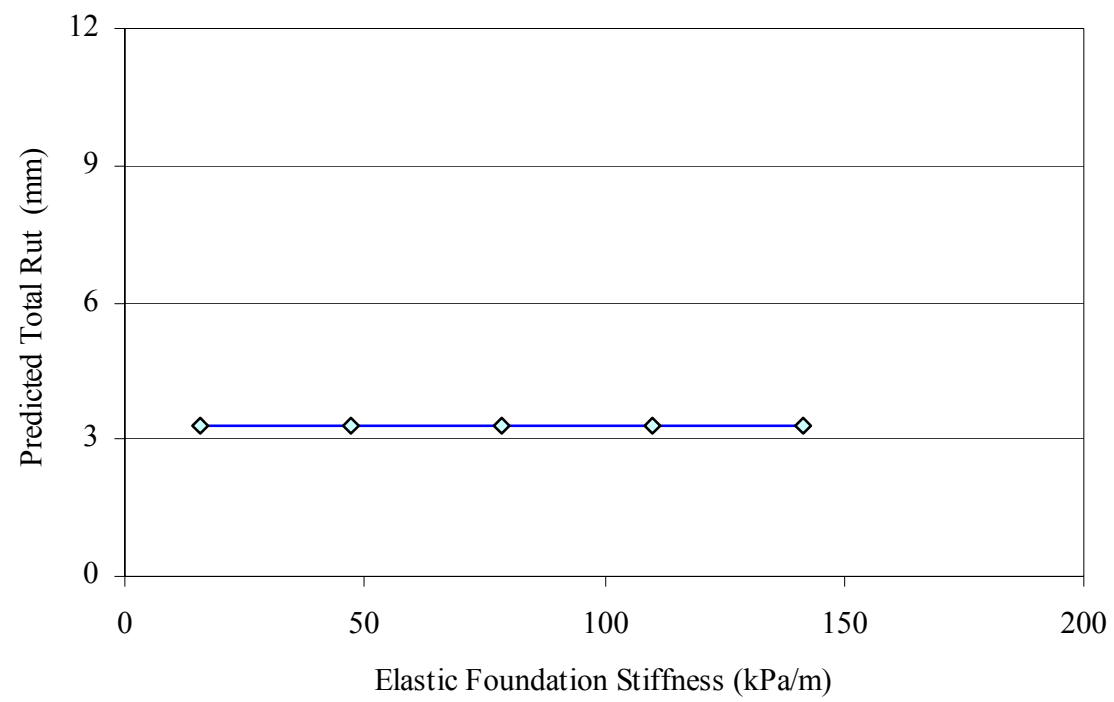

Figure 7-7 Effect of Elastic Foundation Stiffness

\subsubsection{Tire Contact Pressure Distribution}

A decision was made to use uniform tire contact pressure distribution for the loading of the track model based on the fact that the effect of tire contact pressure distribution would be minimized when wheel wander occurs during loading (Hua, 2000). NCAT test track personnel were contacted to obtain the tire print and load data used for the tire contact pressure estimation. They estimated a tire contact pressure of $552 \mathrm{kPa}(80$ psi). This value was subsequently used in the modeling.

An investigation was made to determine the stresses developed due to the loading of the pavement structure. An elastic analysis was conducted using a single wheel assembly. The resulting vertical normal stress is shown as a contour plot in Figure 7-8. The magnitude of the stress gradually decreases down through the pavement. For a 552 $\mathrm{kPa}(80 \mathrm{psi})$ load applied at the pavement surface, the vertical normal stress at the 
subgrade surface level is approximately $69 \mathrm{kPa}(10 \mathrm{psi})$. This low stress level at the subgrade surface is well below the yield strength of the subgrade. Instantaneous plastic strain will therefore not develop in the subgrade under the given loading conditions and the use of an elastic material model for the subgrade is therefore appropriate.

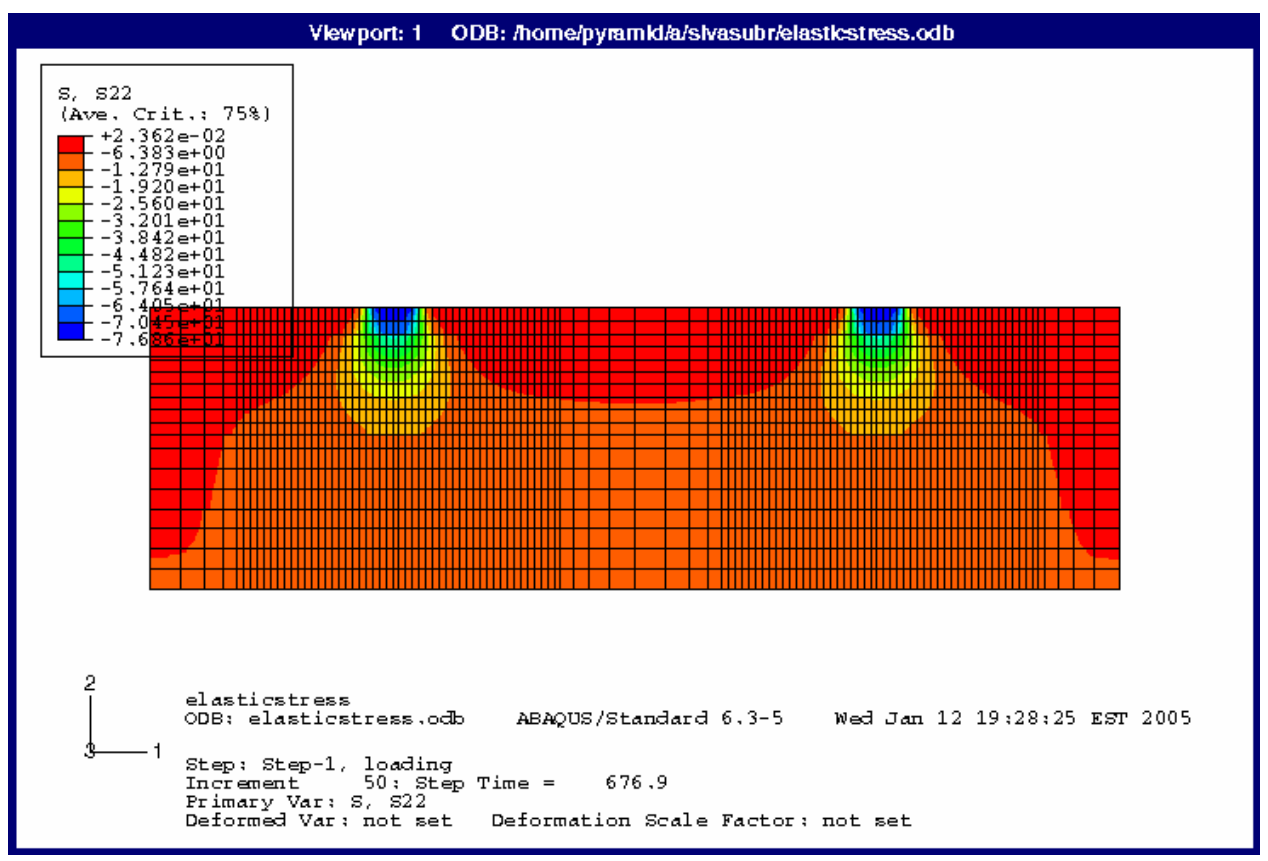

Figure 7-8 Track Pavement Stress Distribution

\subsubsection{Mesh Optimization}

Before any valid conclusions could be drawn, mesh optimization was necessary. A single-axle, single wheel assembly loaded to $552 \mathrm{kPa}$ ( $80 \mathrm{psi}$ ) uniform tire contact pressure as shown in Figure 7-9 was used for the mesh optimization. In this initial analysis, the creep parameters $A$ and $m$ were assigned values of $1.5 \times 10^{-4}$ and -0.84 , respectively. The response was predicted for a loading time of 837 seconds. Four 
different element sizes were investigated to determine the optimal element size in the transverse direction, $100,50,25$, and $12.5 \mathrm{~mm}(4,2,1$, and $0.5 \mathrm{in}$.). The results are shown in Figure 7-10. There was little change in the results as the mesh size changed from 25 to $12.5 \mathrm{~mm}$ (1 to $0.5 \mathrm{in}$.), roughly a one percent change. Considering the required increase in computational time needed for smaller elements, the decision was made to use $25 \mathrm{~mm}$ (1 in.) as the optimal transverse element size.

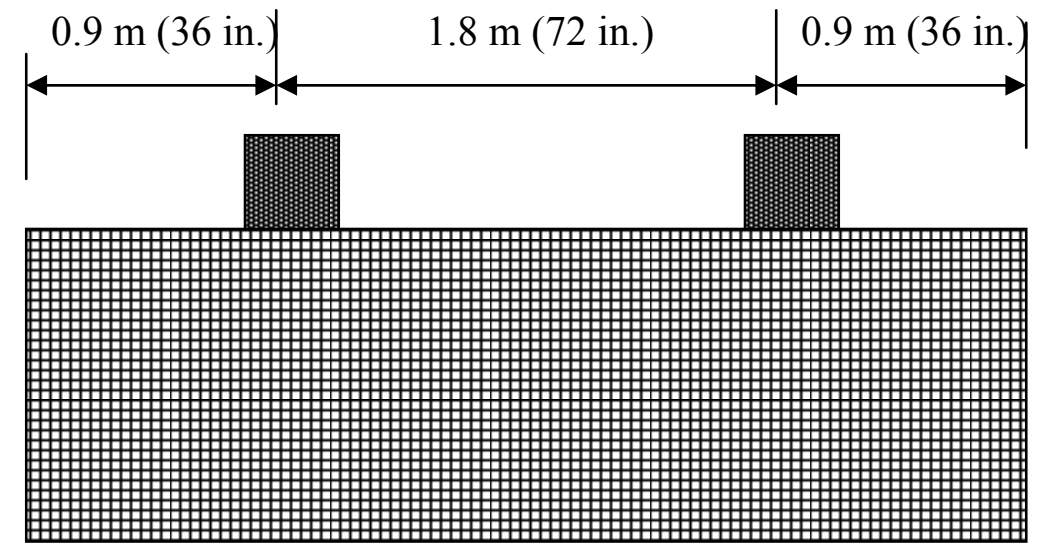

Figure 7-9 Mesh Optimization Loading Configuration

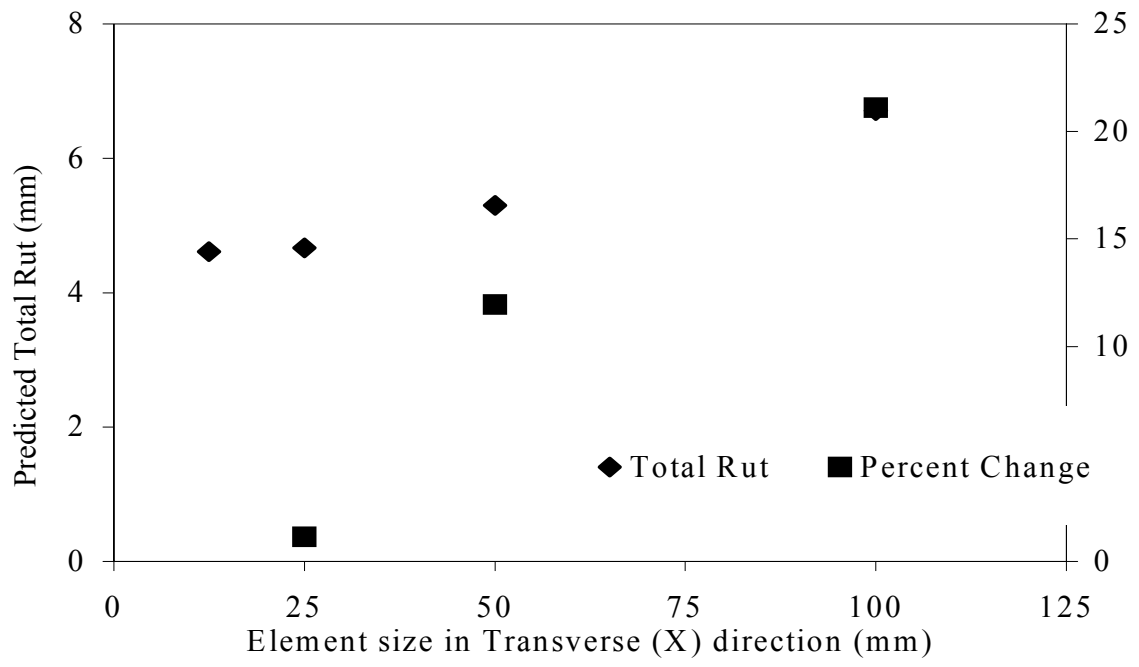

Figure 7-10 Effect of the Transverse Element Size (Test Track) 


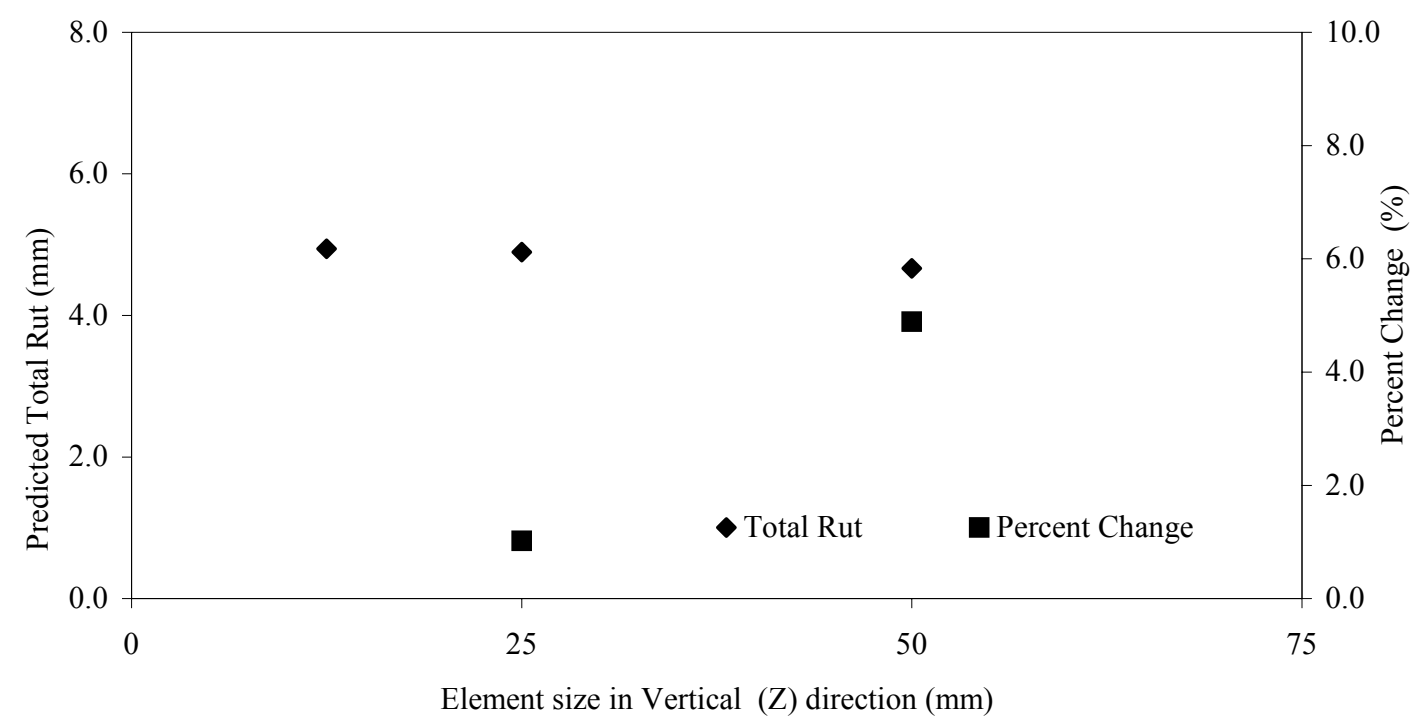

Figure 7-11 Effect of the Vertical Element Size (Test Track)

A similar procedure was repeated to determine the optimum element size in the vertical direction. Figure 7-11 presents the results. As shown, mesh refinement along the y-direction from 25 to $12.5 \mathrm{~mm}$ ( 1 to $0.5 \mathrm{in}$.) again resulted in about a one percent change. The optimal element size was therefore determined to be $25 \times 25 \mathrm{~mm}(1 \times 1 \mathrm{in}$. $)$.

This optimum size was used to model regions where wheel loads were applied. However, away from the loading region, larger elements were used as shown in Figure 712. Smooth element transition was achieved using the bias element sizing option provided in ABAQUS. 

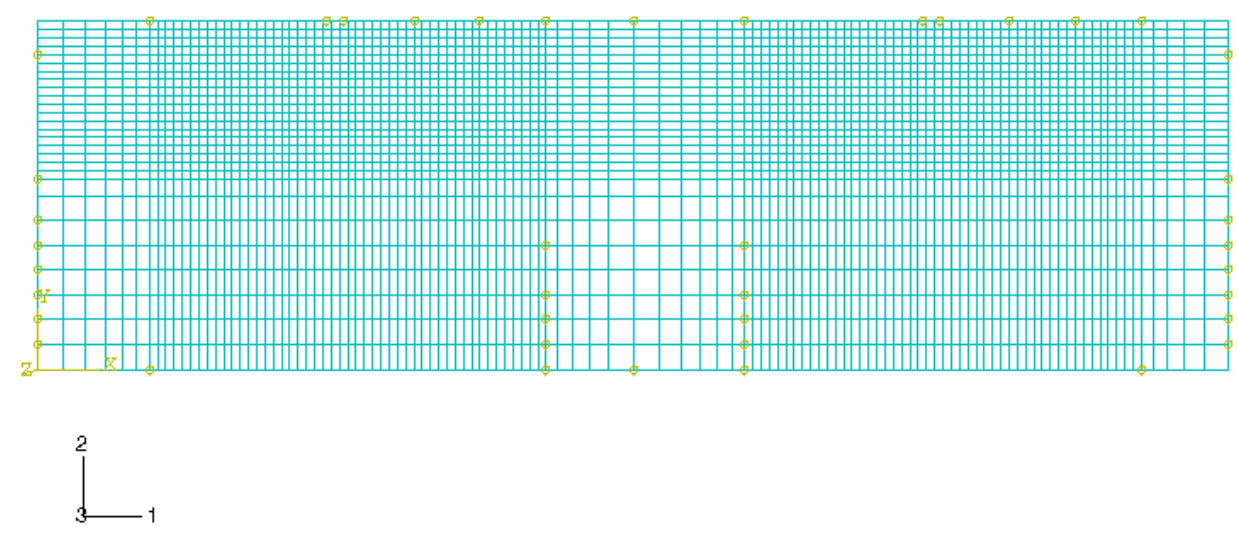

Figure 7-12 Track FEM Mesh

\subsubsection{Modeling Temperature Effects}

Probes installed at different depths in the test track surface layer yielded pavement temperature data and the measurements at the mid-depth of the surface layer $(50 \mathrm{~mm}$ (2 in.) below the surface) were designated as the representative surface layer temperature. These were used as the surface layer temperatures in the FEM work to model the rutting observed at the NCAT test track.

Since rutting is dependent on temperature, the pavement temperature was first superimposed on the track rutting data and is shown in Figures 7-13. The figure indicates that at temperatures below 30C (86F) no rutting occurred. At temperatures between 30 and 40C (86 and 104F) only minimal rutting was observed. A significant amount of rutting occurred when pavement temperatures were above 40C (104F). The data clearly show the effects of temperature on HMA mixture rutting performance. 


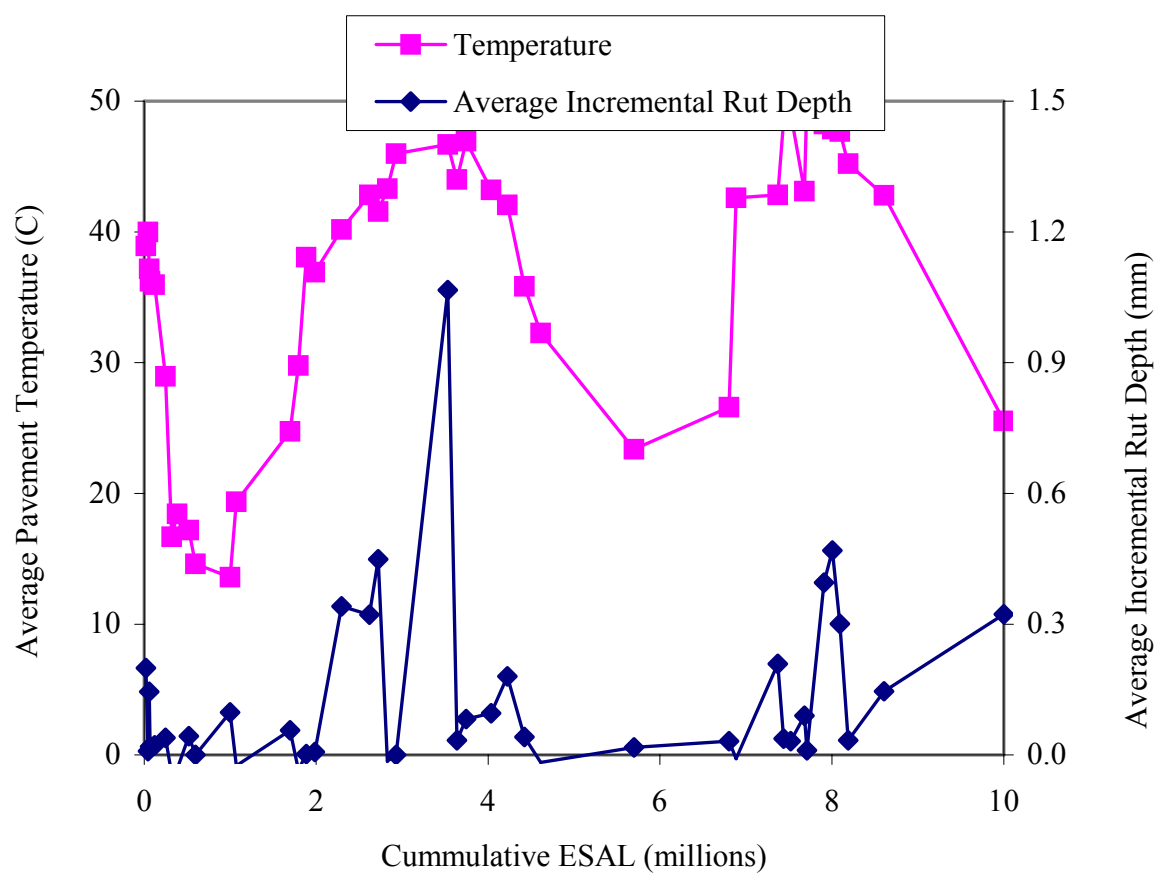

Figure 7-13 Total Rut Development at the NCAT Test Track

In an effort to model the rutting, the total rutting data were separated into seven regions with an average temperature condition assigned to each as illustrated in Figure 714. The analysis regions were designated as:

1. Initial densification (Region I);

2. First summer medium temperature region (Region II);

3. First summer high temperature region (Region III);

4. First winter (Region IV);

5. Second summer medium temperature region (Region V);

6. Second summer high temperature region (Region VI); and

7. Second winter (Region VII). 


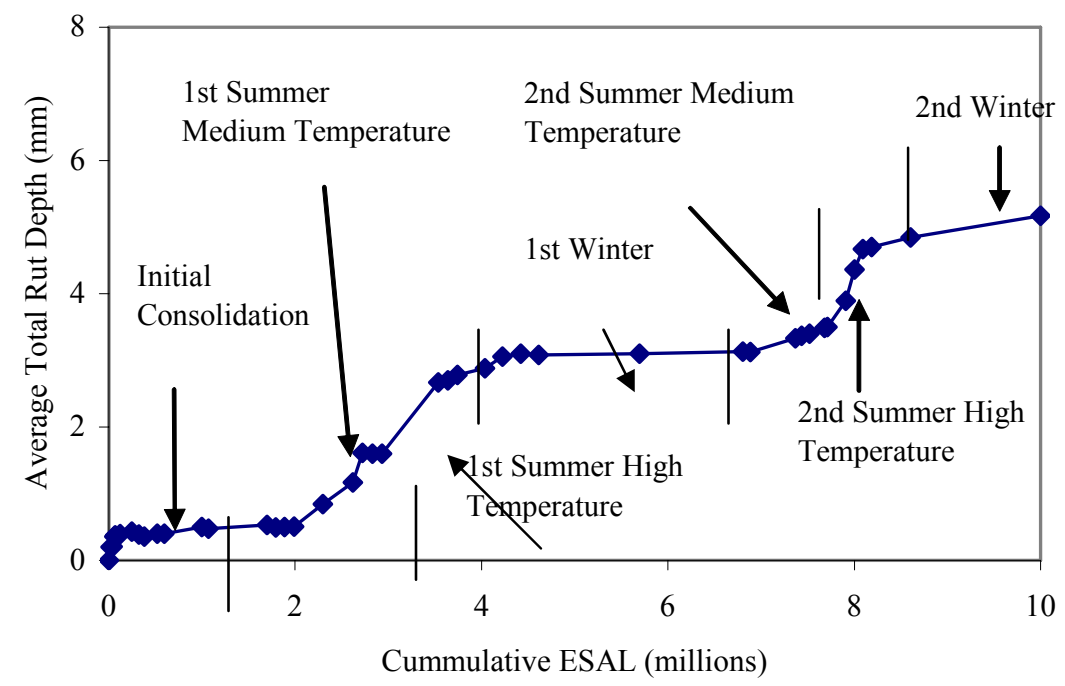

Figure 7-14 Track Rut Depth Development

The initial densification period represents the rutting due to post construction compaction. This rutting is the result of the air voids content reduction in the HMA mixture. The summer rutting development was divided into two separate regions to better simulate the test track temperature conditions and to study the temperature effects on HMA rutting performance. The medium temperature region represents the rutting development during which the average track temperature was 45C (113F). The high temperature region represents the rutting development during which the average track temperature was approximately 50C $(122 \mathrm{~F})$. Two winter periods were also included although little rutting occurred for either period. No efforts were made to model the rutting that developed during the winter periods. This decision was based on the fact that only minimal amounts of rutting developed during the winter and the error in the FEM prediction can be as high as the observed winter rutting. 
FEM techniques were used to model the rutting development for each temperature period at the test track. Since creep material properties are dependent on the pavement temperature, the FEM techniques provided an opportunity to compare the material properties obtained from the APT and PURWheel with the values obtained from the test track model for similar temperatures. APT and PURWheel tests were performed at 50C $(122 \mathrm{~F})$, plus three additional APT test sections were tested at 35C (95F).

\subsubsection{Wheel Wander}

Wheel wander is the distribution of wheel loading in the transverse direction across a pavement. Actual in-service traffic does not follow the same wheel path rather each vehicle can follow a slightly different path. Wander was incorporated into the FEM model using a normal distribution of the total loading time (Kashara, 1982) (Hua, 2000) (Fang, 2000). Normal distribution presents a bell shaped distribution of the loading time with respect to the centerline of the wheel. The cumulative area under the normal distribution function gives an estimate of the total loading time. A normal distribution function has two associated parameters, the average and the standard deviation. In this case, the average of the wheel wander is zero. The standard deviation can be defined by the relationship:

$$
S=\frac{D}{3}
$$

where,

$$
\begin{aligned}
& \mathrm{S}=\text { standard deviation } \text {; and } \\
& \mathrm{D}=\text { maximum wheel wander. }
\end{aligned}
$$


Assuming that the standard deviation is equal to one-third of the maximum wheel wander, $99.74 \%$ of the total loading is included within the area of the normal distribution according to the equation:

$$
2 \times[\Phi([\mathrm{D}-0] /[\mathrm{D} / 3])-0.5]=0.9974
$$

where,

$\Phi(\mathrm{x})=$ value of the normal distribution at $\mathrm{x}$.

Fang (2000) conducted a sensitive study to determine the appropriate maximum wander distance that could be used in FEM analysis. He compared the resulting transverse profiles generated using different wander distances to the transverse profiles obtained from the field and available in the literature. Fang concluded that a maximum wander distance of $0.4 \mathrm{~m}$ (16 in.) was suitable for finite element modeling purposes.

In this project the test track loading was simulated using a single-axle, dual-wheel assembly as shown in Figure 7-15. Hua (2000) developed the following expression to estimate the loading time resulting from the application of dual wheels using superposition principles as shown in Figure 7-16:

$$
\mathrm{T}(\mathrm{X})=[\Phi(\mathrm{X}+(W / 2))-\Phi(\mathrm{X}-(W / 2))] \times \mathrm{T}
$$

where,

$\mathrm{T}(\mathrm{x})=$ loading time for an element located a distance $\mathrm{x}$ from the wheel assembly centerline;

$\Phi(\mathrm{x}+\mathrm{W} / 2), \Phi(\mathrm{x}-\mathrm{W} / 2)=$ value of the normal distribution function;

$\mathrm{W}=$ width of the tire print; and

$\mathrm{T}=$ total loading time. 


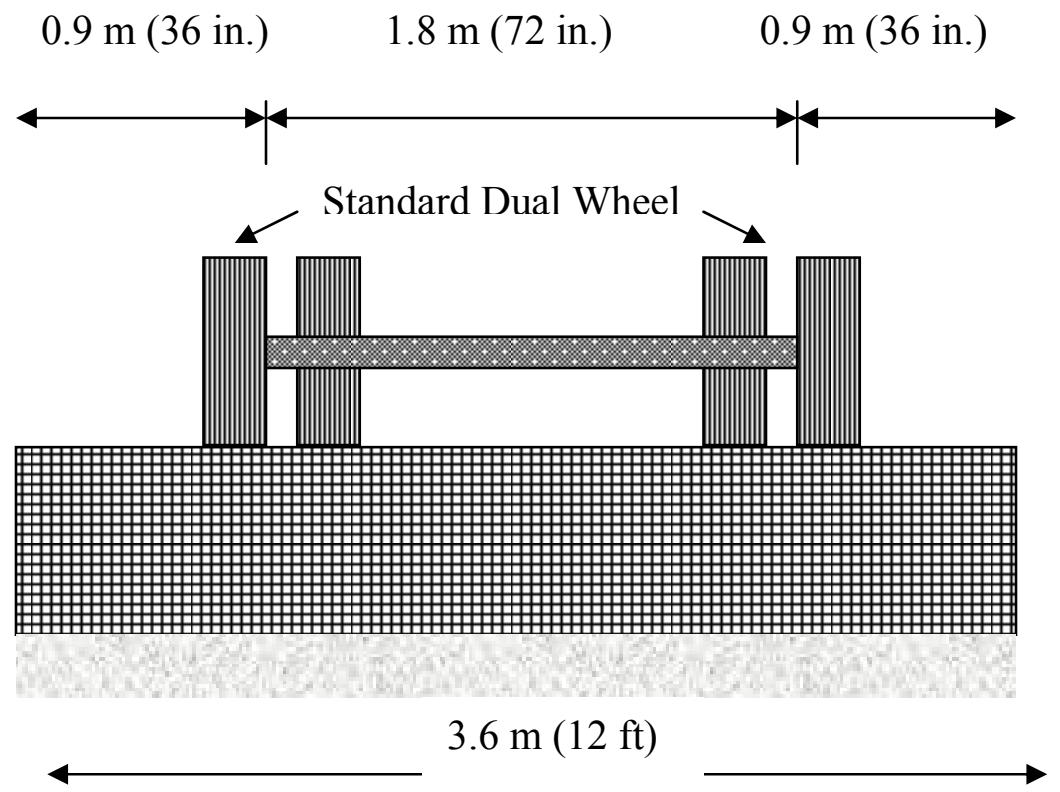

Figure 7-15 Dual-wheel Assembly

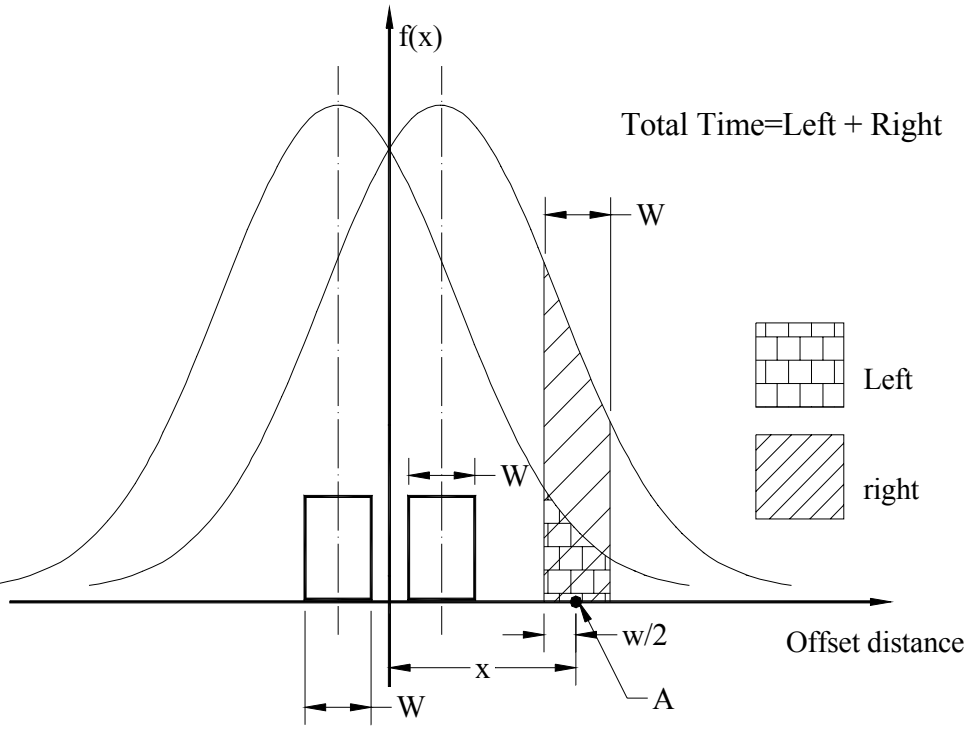

Figure 7-16 Superposition of Dual-wheel Assembly (after Hua, 2000)

The total loading time was estimated based on the tire print length and velocity of the moving traffic along with the number of truck passes. The tire print length used was $0.2 \mathrm{~m}$ ( $8 \mathrm{in}$.) and the speed of the trucks was $72 \mathrm{kph}(45 \mathrm{mph})$. The number of truck 
passes was determined using the fact that a single truck pass applies approximately 10 ESAL. Therefore, to apply 10 million ESAL, one million truck passes would be required. One truck pass would apply approximately 8 wheel passes based on the configuration of the truck-trailer assembly used in the study. Knowing the loading time for a single truck pass, the total loading time was estimated by multiplying it by the number of truck passes to simulate a certain number of ESAL. The distribution of the loading time corresponding to each region is shown in Figure7-17.

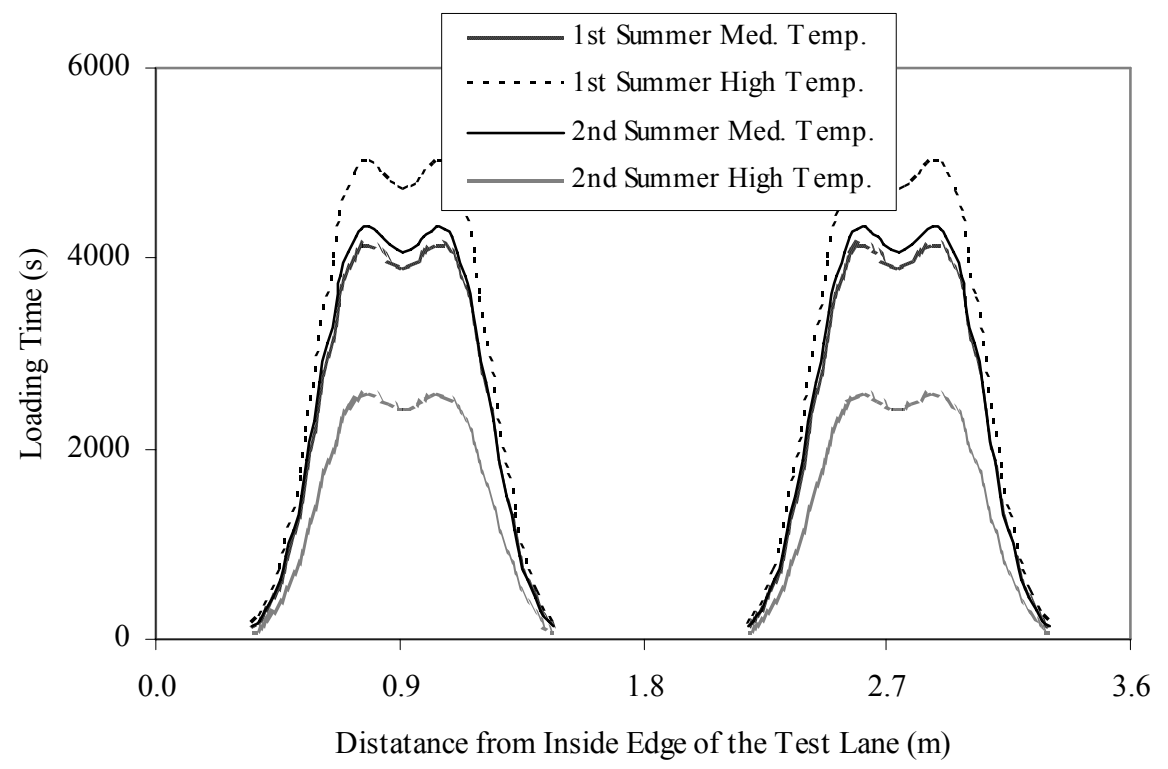

Figure 7-17 Loading Time Distribution for Wheel Wander (Test Track)

\section{$\underline{7.7 \text { APT Modeling }}$}

The APT was also modeled using a two-dimensional model. Hua (2000) developed and compared the responses from two- and three-dimensional modeling of the APT and found that the difference in the predicted response (maximum rut depth) was 
approximately two percent. Two-dimensional modeling was therefore used in this study due to its reasonable accuracy and lower processing time.

\subsubsection{APT Geometry}

As described in Chapter 5, each APT test lanes is $6 \mathrm{~m}(20 \mathrm{ft})$ long by $1.5 \mathrm{~m} \mathrm{(5 \textrm {ft } )}$ wide. Four test lanes were constructed using a single construction cycle. A typical cross section of the APT test pit is shown in Figure 5-1. The traffic application was onedirectional and no wheel wander was applied.

\subsubsection{Element type}

A four-node, bilinear plane strain element (CPE4) was used in the APT modeling. Element nodes and faces are numbered counterclockwise as shown in Figure 7-18. Each nodes is associated with two degrees of freedom, simple translations in the $\mathrm{x}\left(\mathrm{U}_{\mathrm{x}}\right)$ and $\mathrm{y}$ $\left(\mathrm{U}_{\mathrm{y}}\right)$ directions.

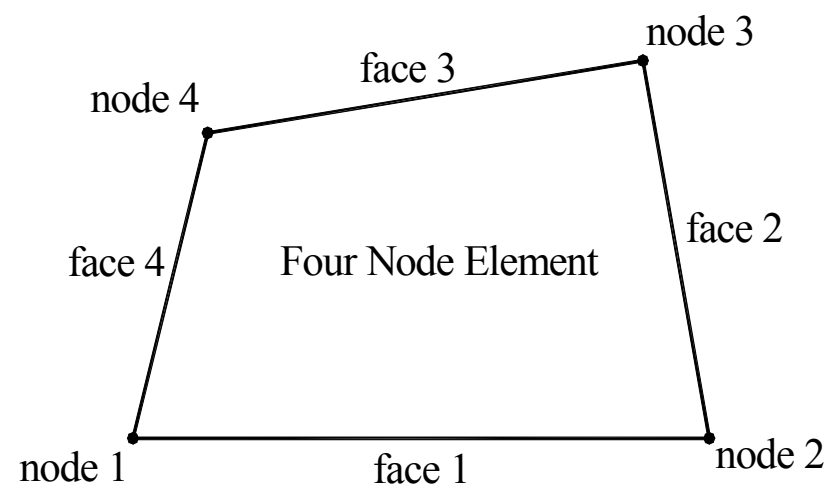

Figure 7-18 Four-node Bilinear Plane Strain Element (CPE4) 


\subsubsection{Boundary Conditions}

Consideration was given to realistic boundary conditions that simulate the actual APT test conditions. Figure 7-19 shows the boundary conditions used in the APT modeling. A fixed boundary condition was assigned at the bottom of the APT test section. The translations in the vertical and transverse direction were restrained.

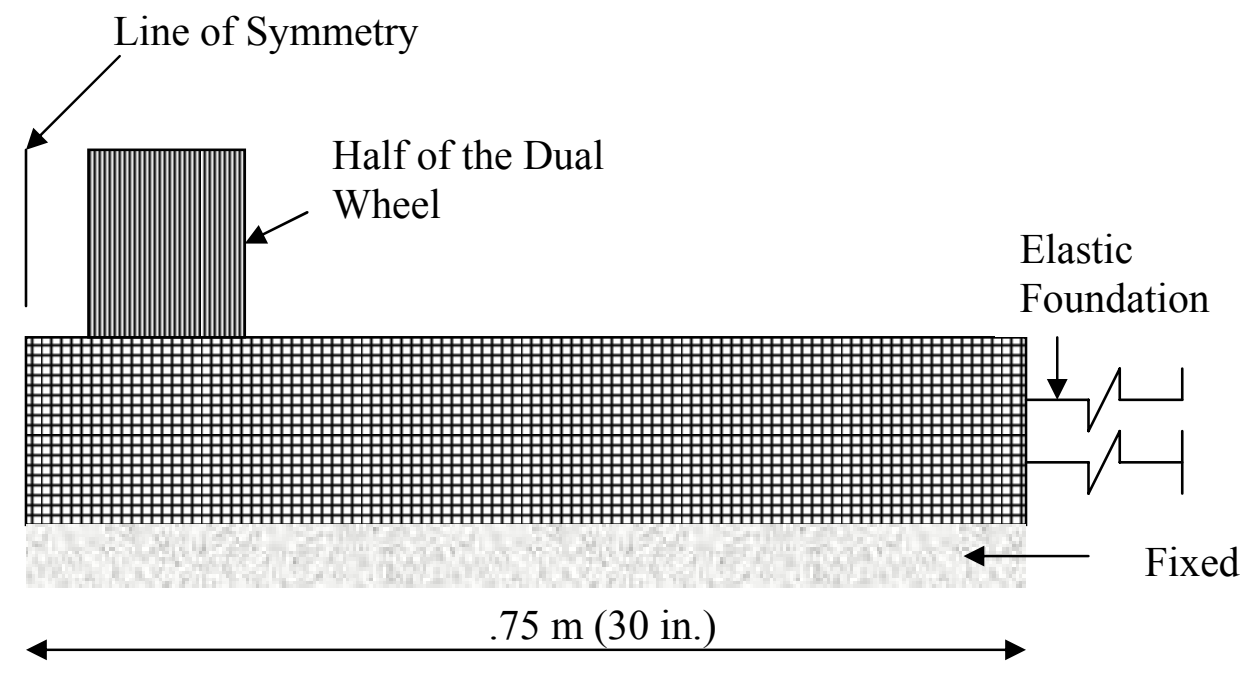

Figure 7-19 APT Boundary Conditions

Realizing that the geometry and the loading are symmetrical about the centerline of the test lane, an axi-symmetric boundary condition was imposed to represent the centerline of the APT test lane and only one-half of the test lane was analyzed. This simplification resulted in less computational time without reduction in the accuracy of the results. To simulate the passive reaction of the adjoining test lane, an elastic foundation was used to represent the common face of the test lane. 
A sensitivity study was conducted to determine the effect of elastic foundation stiffness on rutting performance. Figure 7-20 shows the total rut depth as a function of the elastic foundation stiffness. As shown, the effect of the foundation stiffness on total rut depth is negligible and a $78.5-\mathrm{kPa} / \mathrm{m}(500-\mathrm{pcf})$ foundation stiffness was subsequently used in the model.

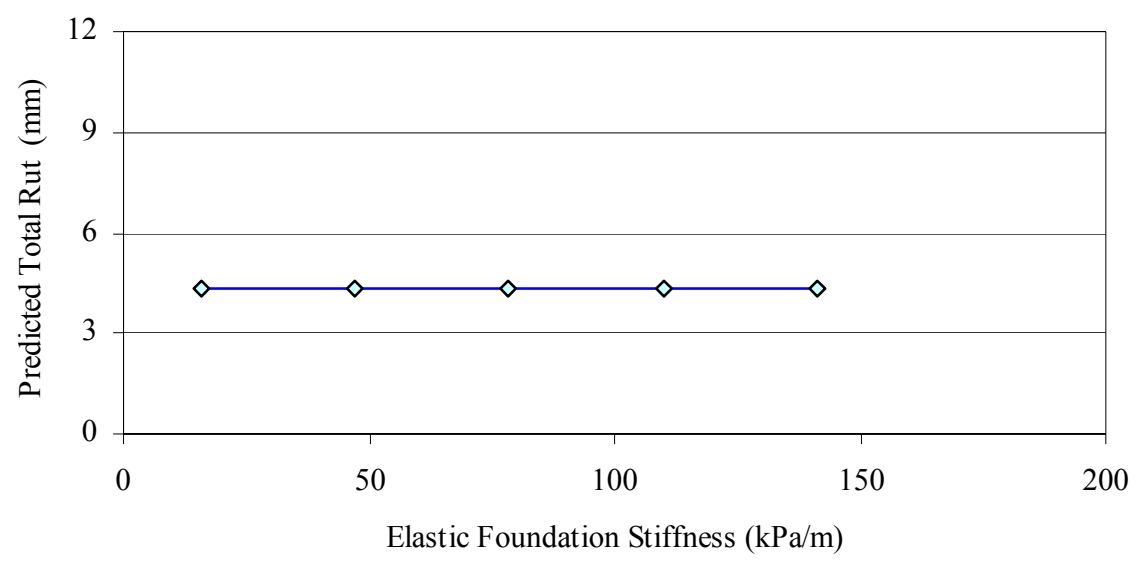

Figure 7-20 Effect of Elastic Foundation Stiffness

\subsubsection{Pressure Distribution}

In order to estimate pavement responses due to traffic loading on an HMA pavement, it is common practice to assume that the vertical stresses induced by the traffic are uniformly distributed over a circular area (Huang, 1993). The magnitude of the stress is assumed to be equal to that of the tire inflation pressure or slightly lower. However, in reality and according to existing literature, the vertical stresses at the tire/pavement interface are not uniformly distributed and shear stresses are also present. Additionally, it 
is difficult to quantify the three-dimensional stresses generated by moving tires on pavements.

Hua (2000) modeled the effect of using different tire pressures and application areas on the rutting performance. He compared a uniform tire pressure applied over a rectangular area, uniform tire pressure applied over an equivalent tire tread, and nonuniform tire pressure applied over an equivalent tire tread area. The equivalent tire tread area was obtained by converting the measured tire print into equivalent rectangles. Based on a comparison between the observed and predicted rutting profiles, he concluded that non-uniform tire pressure with equivalent tire treads gave the best fit. This is the case for the APT loading without wheel wander. Therefore, non-uniform tire pressure and an equivalent tire tread area were used in this study. Non-uniform tire pressure distribution was estimated based on the data from a previous study (Debeer and Fisher, 1997).

\subsubsection{Vehicle-Road Surface Pressure Transducer Array}

The University of California conducted a study at their accelerated pavement testing facility to find the three-dimensional stress distribution under a moving load (Debeer and Fisher, 1997). Measurements were taken using the Vehicle-Road Surface Pressure Transducer Array (VRSPTA) for different types of tires and load combinations. The VRSPTA consists of 1,041 flat-topped, cone-shaped, hollow steel pins mounted on a steel base plate. The steel pins were mounted in 51 rows and the 20 center row pins were instrumented with strain gauges and used as tri-axial load cells. The remaining pins were supporting pins with the same shape, contact area, and stiffness as that of the 
instrumented pins. The VRSPTA surface was flush with the pavement surface. A layout of the VRSPTA is presented in Figure 7-21.
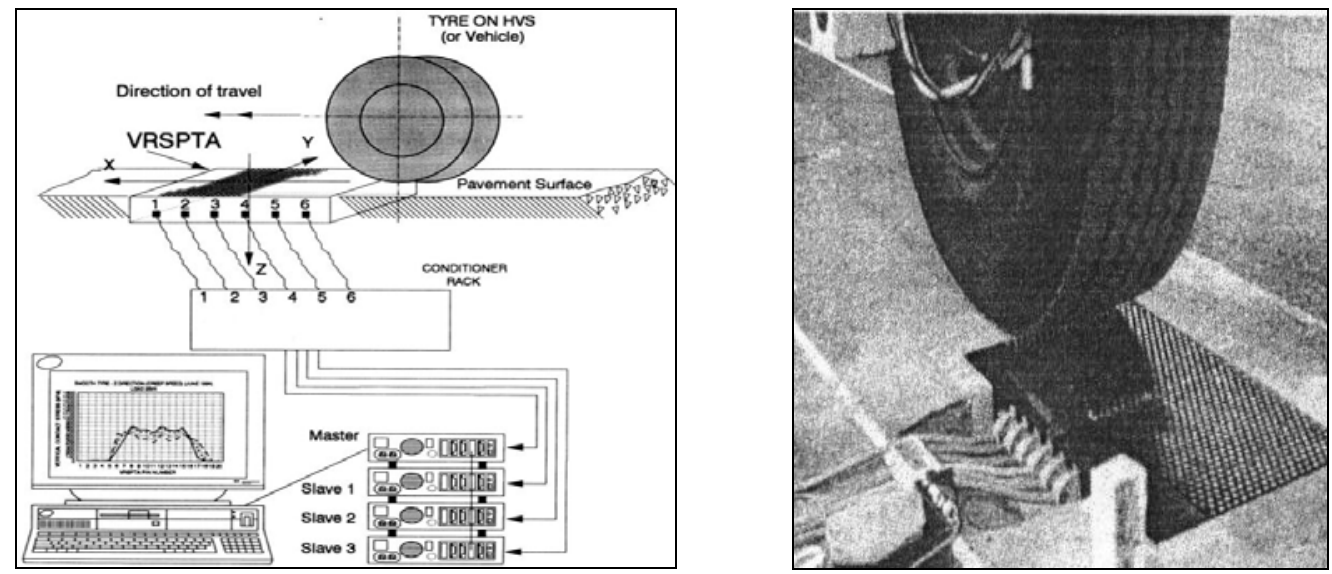

Figure 7-21 Layout of the VRSPTA System (After Hua, 2000)

As the wheel load moves across the steel plate of the VRSPTA, the loads are measured using the load cells in one line across the tire contact patch. Sixty active channels (20 pins $\times 3$ directions) were used to scan strain gauges when the load moved over the instrumented pins and data acquisition was triggered by the coaxial cables and/or by the optical beams located on the approach side of the VRSPTA. The loads were measured in the vertical, transverse, and longitudinal directions. Using the load and the effective area, the stresses were estimated. The effective area for one pin was estimated using a diamond shape as shown in Figure 7-22. Note the diagonal configuration of the instrumented pins and the support pins, which resulted in a diamond-shaped effective area corresponding to a single instrumented pin. Typically, a sampling rate of $150 \mathrm{~Hz}$ was used for each channel. 


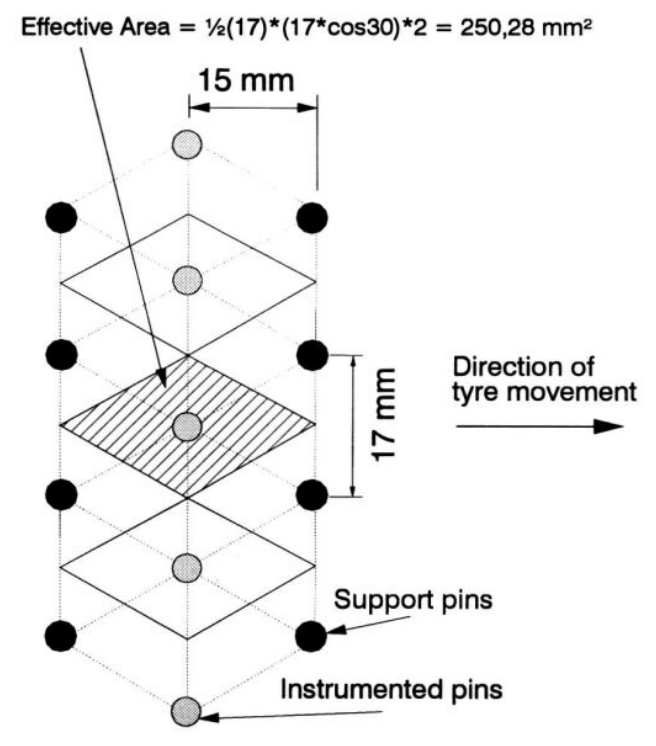

Figure 7-22 VRSPTA Diamond-shaped Effective Area (After Hua, 2000)

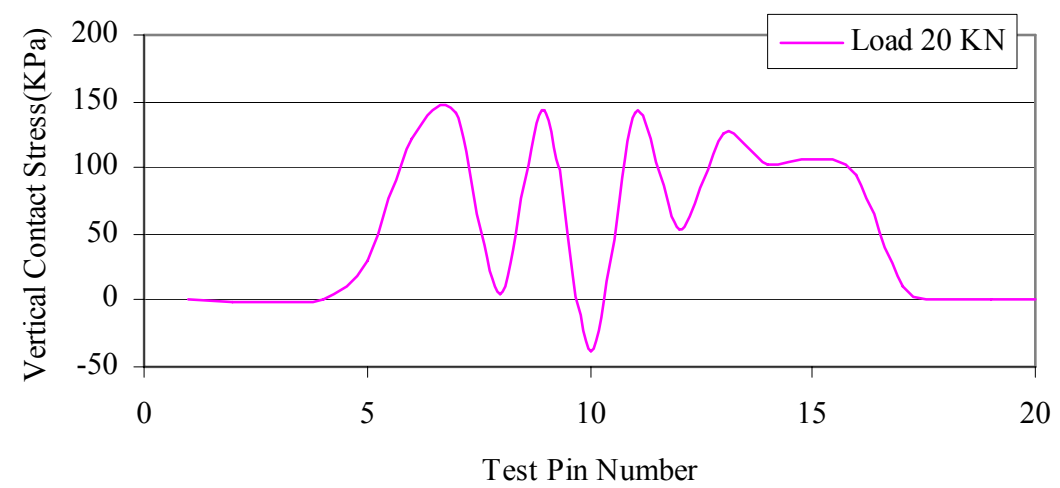

Figure 7-23 Contact Stress Distribution for Goodyear 11R22.5 (Tire Pressure $=620 \mathrm{kPa}$ ) (Debeer and Fisher, 1997)

VRSPTA data were used to estimate the actual pavement/tire interface vertical stresses in the FEM model of the APT by extrapolating data from the University of California study. The APT uses G159 A R11.5 tires so the California data were searched for contact stress measurements for a similar type of tire, tire inflation pressure, and load (20 $\mathrm{kN}$ for this testing). Information on the tire type and inflation pressure were available, 
but data was not available for $20 \mathrm{kN}$ load. The needed information was therefore extrapolated from the available data of 26 and $31 \mathrm{kN}$. The stress ratio was assumed to be equal for the VRSPTA and APT loading. The stress ratio (1.16) was obtained using the three-dimensional stress plot from the VRSPTA data as shown in Figure 7-23. Actual tire print data obtained from the APT is presented in Figure 7-24. This was converted into the equivalent rectangular tire print as shown in Figure 7-25. Knowing the loads, contact area (converted tire print data from Figure 7-24) and the stress ratio, the vertical stresses were estimated as:

$$
\begin{gathered}
\mathrm{P} 2 / \mathrm{P} 1=1.16 \\
20.94 \mathrm{P} 1+27.87 \mathrm{P} 2=4400
\end{gathered}
$$

where P1 is the actual contact stress on the two outside rectangles and P2 is the actual contact stresses on the middle three rectangular of the equivalent APT tire shown in Figure 7-25. Solving for P1 and P2, and converting to $\mathrm{kPa}$ yields $\mathrm{P} 1=570 \mathrm{kPa}(82.6 \mathrm{psi})$ and $\mathrm{P} 2=661 \mathrm{kPa}(95.8 \mathrm{psi})$. These pressures are shown in Figure 7-26 and used in the FEM modeling.

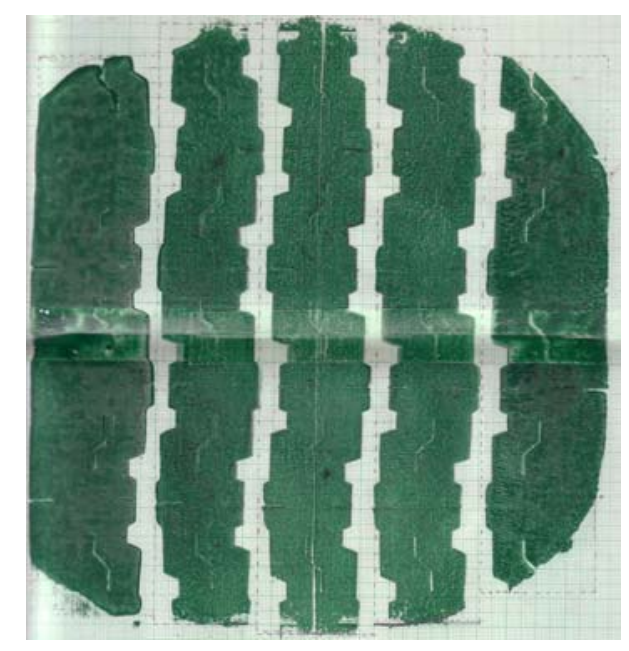

Figure 7-24 APT Tire Print 


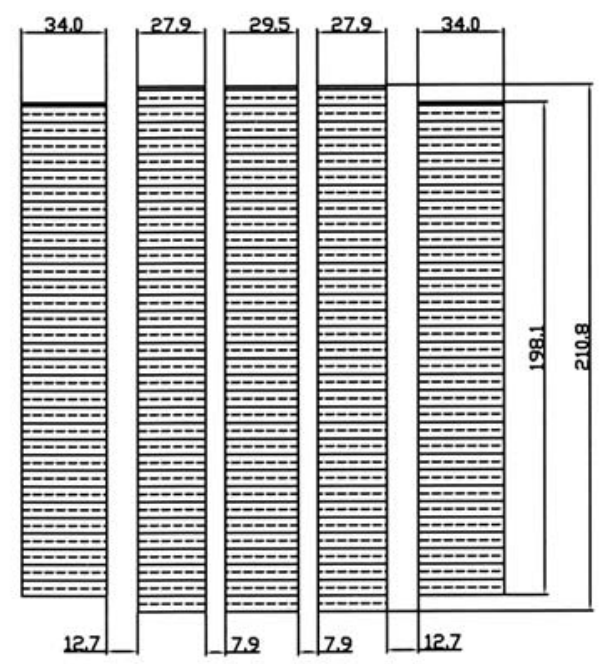

Figure 7-25 Equivalent APT Tire Print

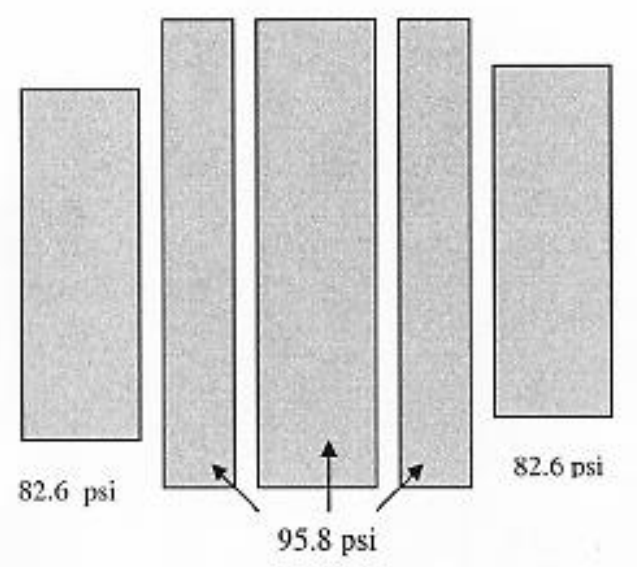

Figure 7-26 APT Contact Pressure Distribution

\subsubsection{Loading Time}

As described in section 7.5, the APT loading was simulated using a simplified loading method with the cumulative loading time estimated using the tire print length, the 
wheel velocity and the number of wheel passes. Since the equivalent tire print lengths of the APT have two measurements as shown in Figure 7-25, two loading times (one each for the middle three and the outer two prints) were estimated and used in the modeling and calculation processes. The average wheel velocity of the APT is $8 \mathrm{kph}(5 \mathrm{mph})$ and the tire print lengths are as shown in Figure 7-25. The loading time inputs corresponding to 10,000 wheel passes were 943 and 886 seconds for the inner and outer prints, respectively.

\subsubsection{Mesh Optimization}

An initial analysis was conducted to determine the optimum size for the elements in the APT FEM modeling. For the initial analysis the creep parameters A and $\mathrm{m}$ were assigned values of $1.5 \times 10^{-4}$ and -0.84 , respectively. The total rut depth was predicted using 10,000 wheels passes on a $75 \mathrm{~mm}$ (3 in.) thick APT test lane. The symmetry of the APT resulted in only one-half of the APT test lane slab needing to be modeled. As an initial attempt, the element with a varying size in the transverse direction (along the $\mathrm{X}$ axis) was investigated while the element size in the vertical direction (along $\mathrm{Z}$ axis) was held fixed. Figure 7-27 shows the variation of the predicted total rut with the size of the element in the transverse direction. Note that reducing the element size from $3.81 \mathrm{~mm}$ (0.15 in.) to $2.54 \mathrm{~mm}(0.1 \mathrm{in}$.) does not result in a substantial change in the total rut depth. Considering the computational time increment and the observed minimal change in the response, a decision was made to use $3.81 \mathrm{~mm}(0.15 \mathrm{in}$.$) as the optimal size in the$ transverse direction. 


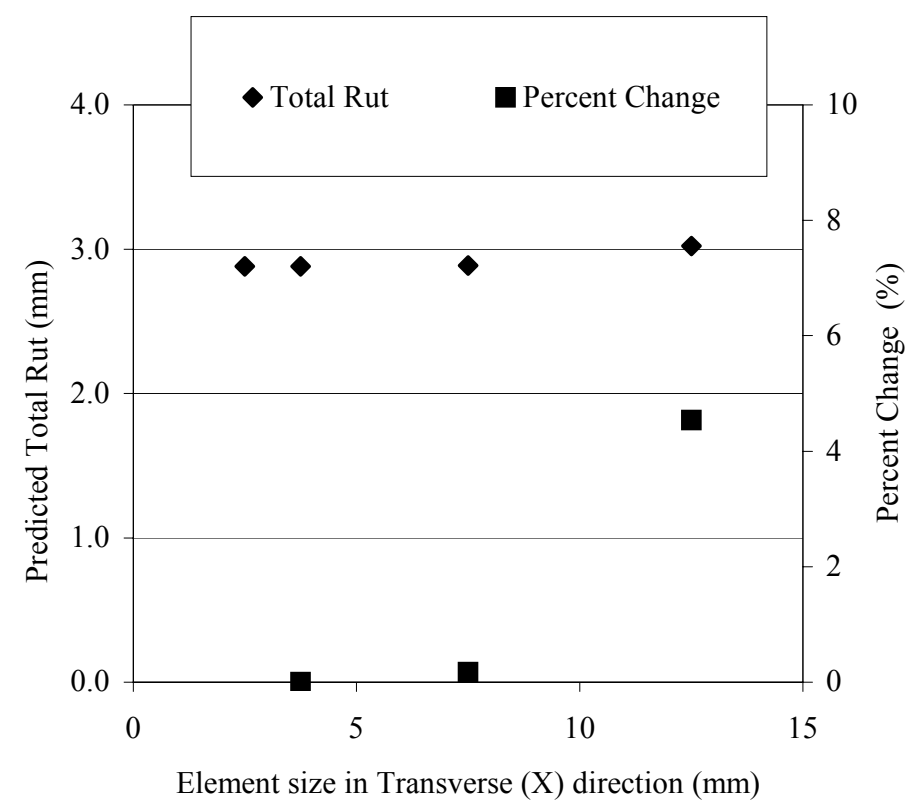

Figure 7-27 Effect of Transverse Direction Element Size (APT)

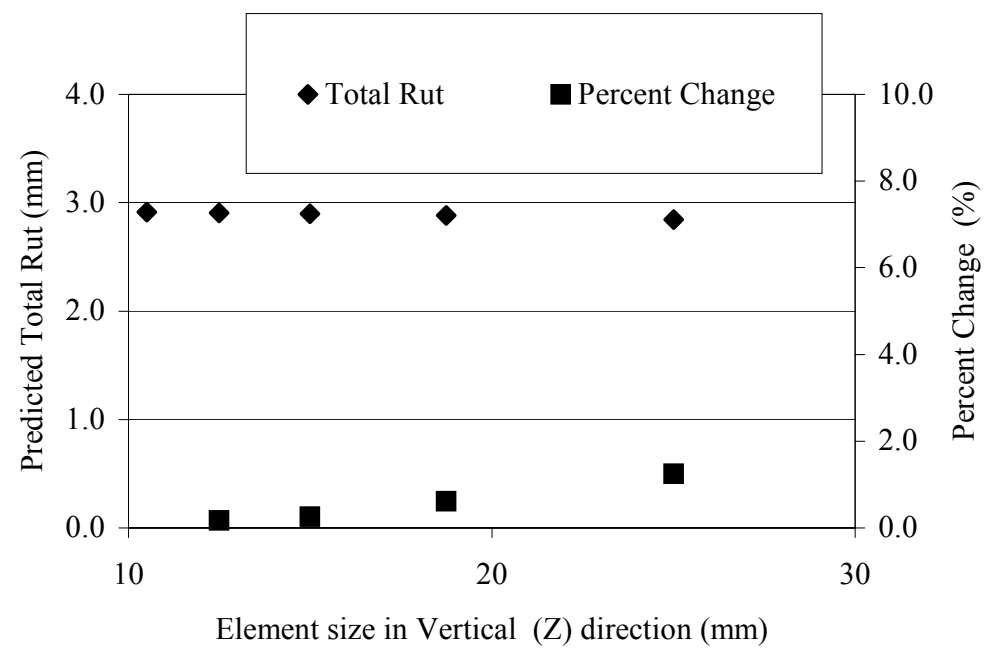

Figure 7-28 Effect of Vertical Direction Element Size (APT) 
A similar procedure was repeated to determine the optimum size along the vertical (Y) direction. Figure 7-28 presents the variation of the total rut with the varying element sizes in the vertical direction. As shown, mesh refinement along the Y-direction from $12.5 \mathrm{~mm}(0.5 \mathrm{in}$.) to $10.7 \mathrm{~mm}$ (0.42 in.) resulted in only about $0.2 \%$ improvement. Combined with the transverse analysis explained above, the optimal element size was determined to be $12.5 \times 3.81 \mathrm{~mm}(0.5 \times 0.15 \mathrm{in}$. $)$.

Figure 7-29 shows the finite element mesh used to model the APT. Smaller elements were used in the high stress areas while larger elements were used away from the high stress areas.
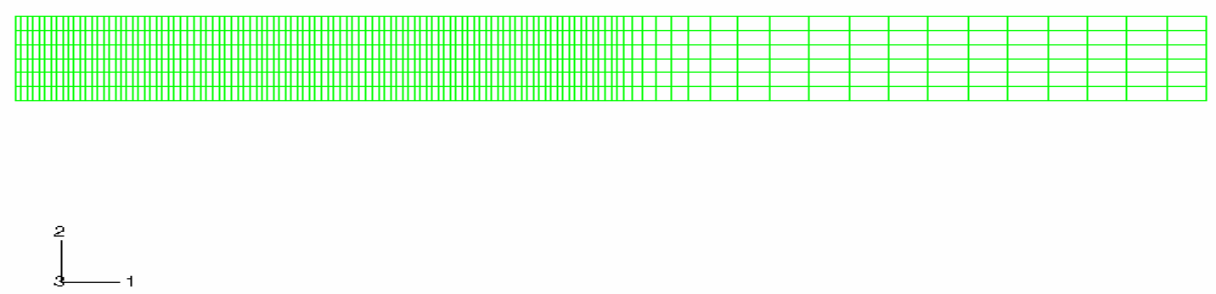

Figure 7-29 APT Finite Element Mesh

\subsection{PURWheel Modeling}

\subsubsection{Test Specimen Geometry}

Figures 7-30 and 7-31 show the plan and cross section details of a PURWheel specimen (slab). The thickness of the slabs varies from 50 to $100 \mathrm{~mm}$ ( 2 to 4 in.). The average slab width is approximately $292 \mathrm{~mm}$ (11.5 in.) while the average slab length is approximately $305 \mathrm{~mm}$ (12 in.). In the modeling, these average dimensions were used for 
the slab width and length. However, the actual thicknesses of the slabs were used to account for the effect of the thickness on rutting. A previous study by Hua (2000) showed that the effect of the slab length on the rutting magnitude is not significant and that the maximum variation of rut depth (compactive rut) is less than one percent.

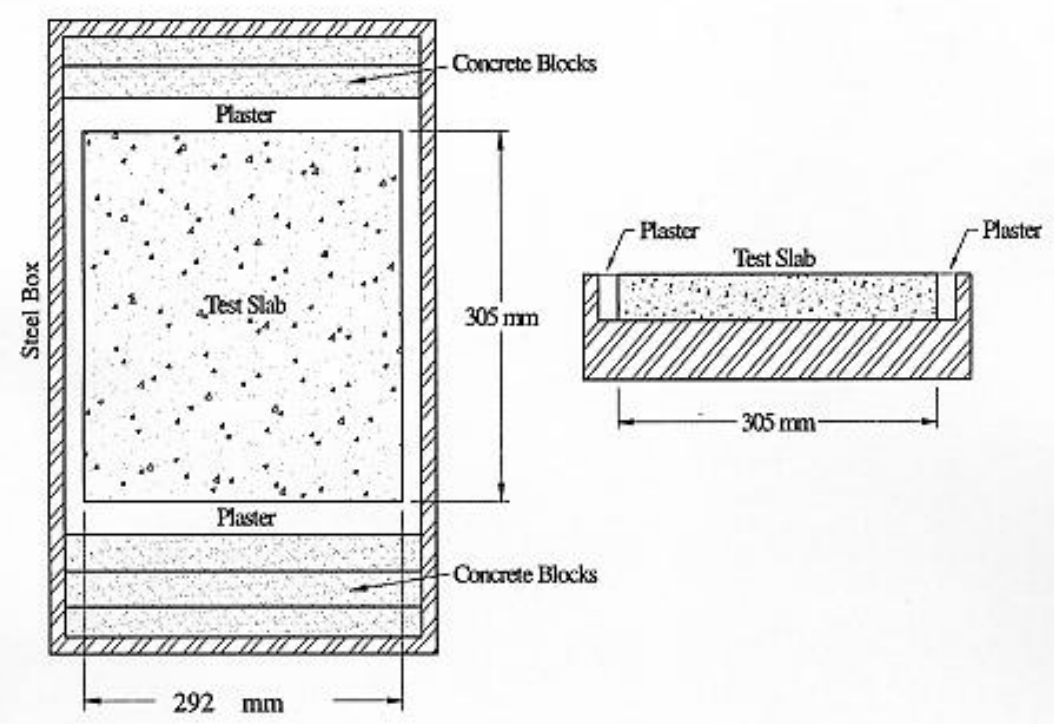

Figure 7-30 PURWheel Specimen Geometry (After Hua, 2000)

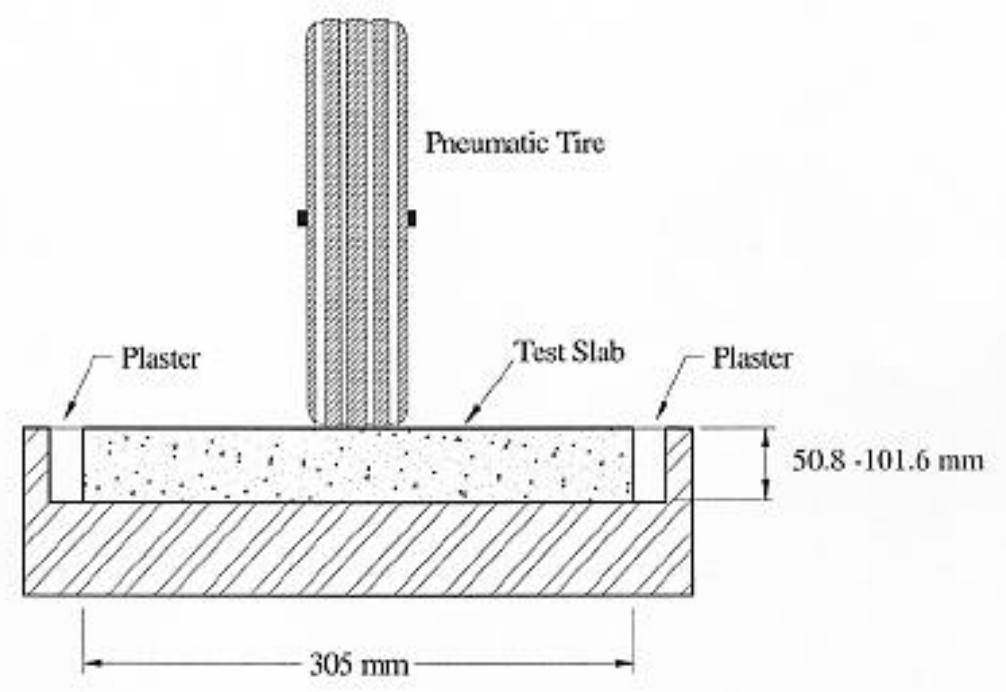

Figure 7-31 PURWheel Loading Configuration (After Hua, 2000) 


\subsubsection{Element Type}

Since the PURWheel slab was modeled as a three-dimensional problem, the ABAQUS solid element type was used. Based on the experience of previous applications by Zaghloul et al. (1993), Huang (1995), Pan (1997), and Hua (2000), an eight-node, linear brick element (reduced integration) (C3D8R) was selected for use. A schematic of the element is shown in Figure 7-32. The nodes are numbered in the counterclockwise direction and each node has three degrees of freedom, translations in the $\mathrm{x}, \mathrm{y}$, and $\mathrm{z}$ directions.

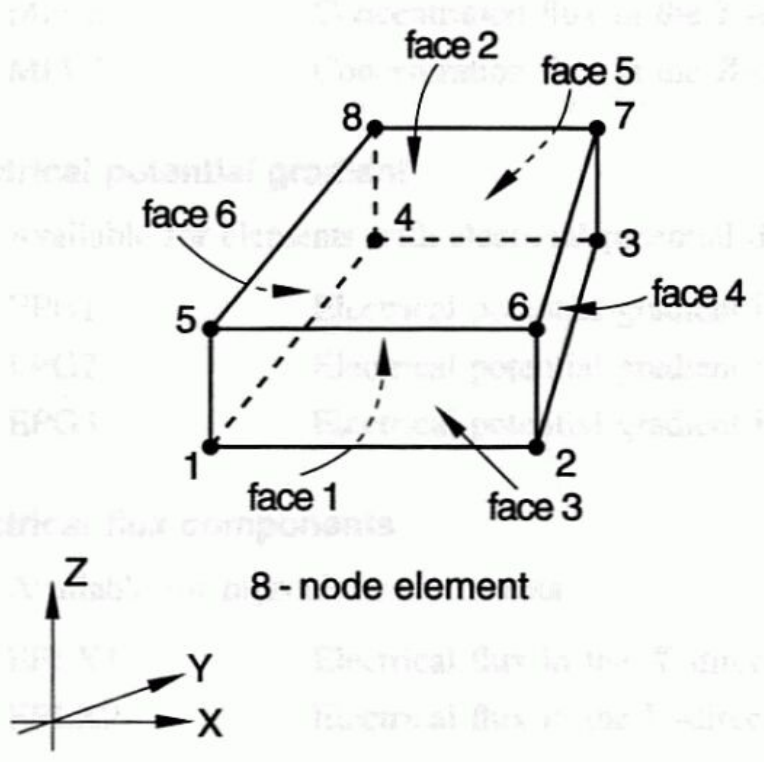

Figure 7-32 Eight-node Linear Brick Element, C3D8R (After Hua, 2000)

\subsubsection{Boundary Conditions}

The PURWheel test specimens are held rigidly in the mounting box and therefore fixed boundary conditions are applicable. 


\subsubsection{Tire Contact Pressure Distribution}

For realistic modeling of the PURWheel loading it was necessary to estimate the actual contact stresses generated by a given load for different tire tread patterns. Unfortunately, no research data of the actual contact stresses is available for the PURWheel loading. However, efforts were made in the modeling process to get a best estimate of the contact stress based on the actual tire print area rather than the gross contact area. The contact pressure for the PURWheel was estimated using the actual tire prints and wheel load.

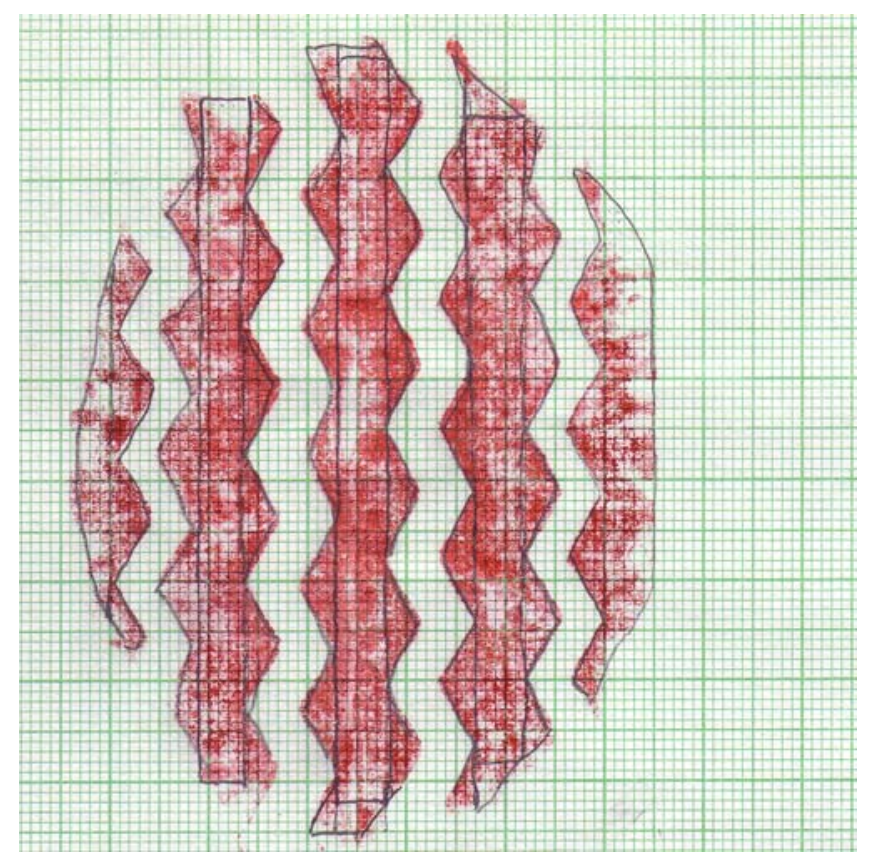

Figure 7-33 PURWheel Tire Print

The actual PURWheel tire print contains a zigzag pattern of tire treads as shown in Figure 7-33. This was transformed into an equivalent rectangular area as shown in Figure 7-34 (Hua, 2000). Since no research data for PURWheel contact stresses were available, a uniformly distributed contact stress was assumed in the modeling with the 
contact area estimated based on the transformed equivalent rectangular area of the PURWheel tire print as indicated in Figure 7-35. The actual contact pressure based on the tire print was $681.7 \mathrm{kPa}(98.8 \mathrm{psi})$ while the target contact pressure on the PURWheel was $690 \mathrm{kPa}(100 \mathrm{psi})$.
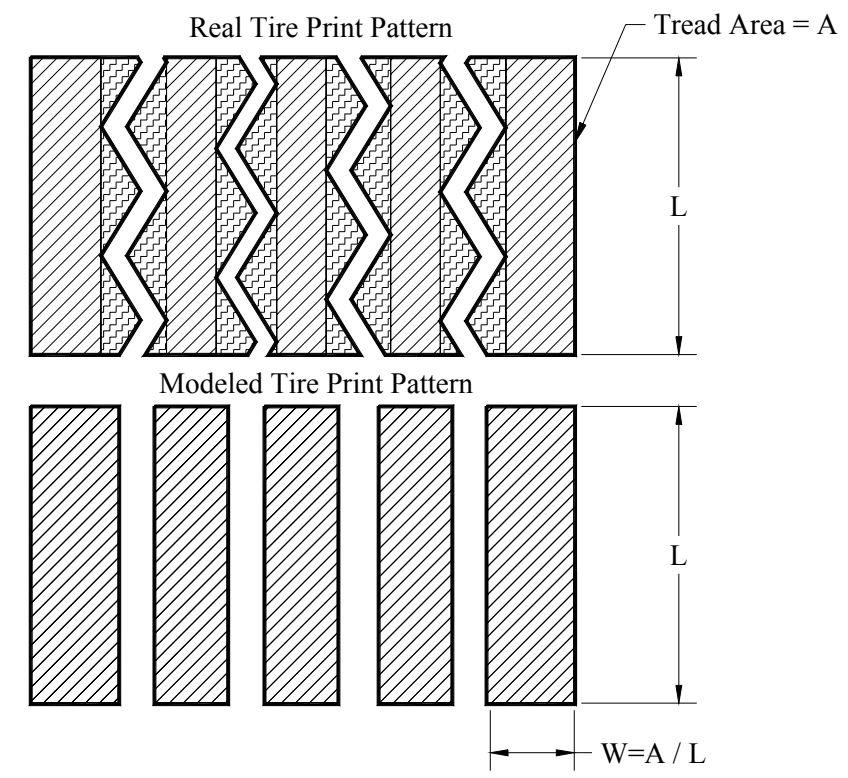

Figure 7-34 PURWheel Tire Print Conversion (After Hua,2000)

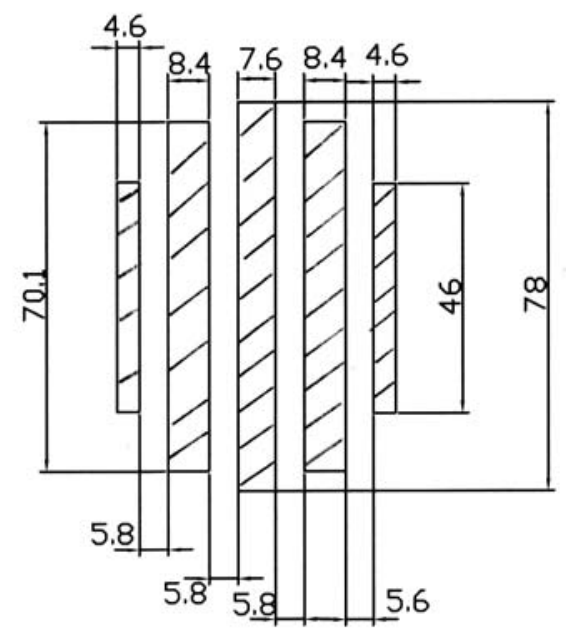

Figure 7-35 PURWheel Equivalent Tire Print Data 


\subsubsection{Loading Time}

The PURWheel loading was simulated using the simplified loading method already described. The length of the tire print was divided by the wheel speed to determine the load time corresponding to a single wheel pass. The individual computed load time was then multiplied by the number of wheel passes to obtain the cumulative loading time. For the PURWheel, the rut development for 10,000 wheel passes was obtained and compared with the experimental data in the calculation procedure. Since the equivalent tire print lengths of the PURWheel have three measurements as shown in Figure 7-35, three loading times were estimated and used in the modeling and calculation process. The average wheel velocity of the PURWheel was $33 \mathrm{~cm} / \mathrm{s}(13 \mathrm{in} . / \mathrm{s})$, and the details of the tire print lengths are shown in Figure 7-35. The estimated loading time inputs in the model corresponding to 10,000 wheel passes were 2,360 (center), 2,120 (between the middle and outside), and 1,390 seconds (outside).

\subsubsection{Mesh Optimization}

For the initial optimization analysis parameters $\mathrm{A}$ and $\mathrm{m}$ were assigned values of $10^{-4}$ and -0.88 , respectively. The total rut depth was predicted for 10,000 wheel passes. The symmetry of the PURWheel loading and geometry resulted in only one-quarter of the slab being modeled with symmetrical boundary conditions assigned on the center of the slab along the transverse and longitudinal directions as shown in Figure 7-36.

Results to determine the optimum element size for three directions are shown in Figures 7-37 through 7-39. Based on the analyses, the optimal element size was determined to be $2.54 \times 10.16 \times 10.16 \mathrm{~mm}(0.1 \times 0.4 \times 0.4$ in. $)$. Figure $7-40$ shows the plan 
view of the finite element mesh used in the analysis while Figure 7-41 shows the elevation view of the mesh. As shown in Figures 7-40 and 7-41, finer elements were used in the potential high stress regions (loading area) and the element sizes were gradually increased away from the high stress regions.

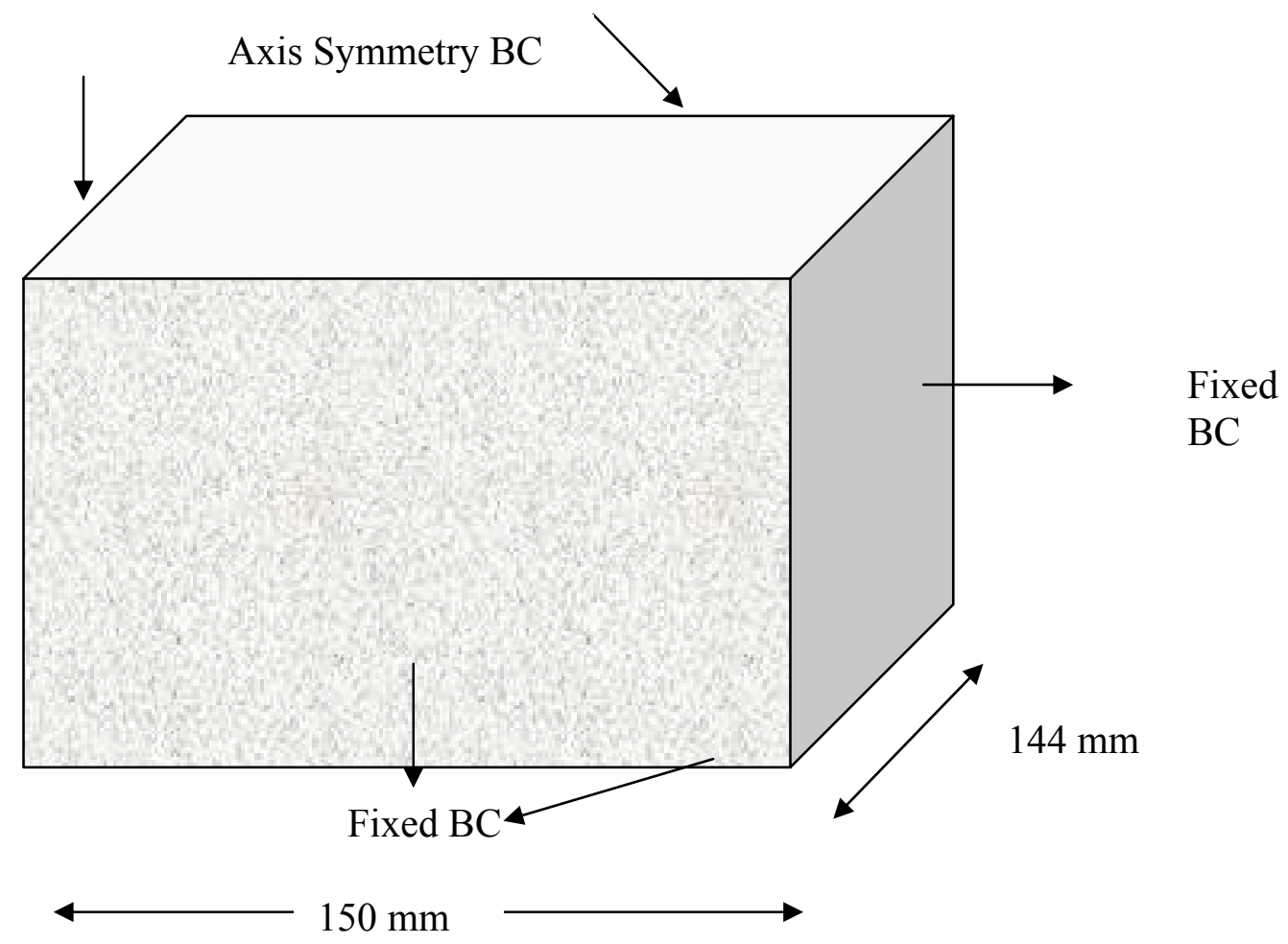

Figure 7-36 PURWheel Slab (quarter size) used in the FEM 


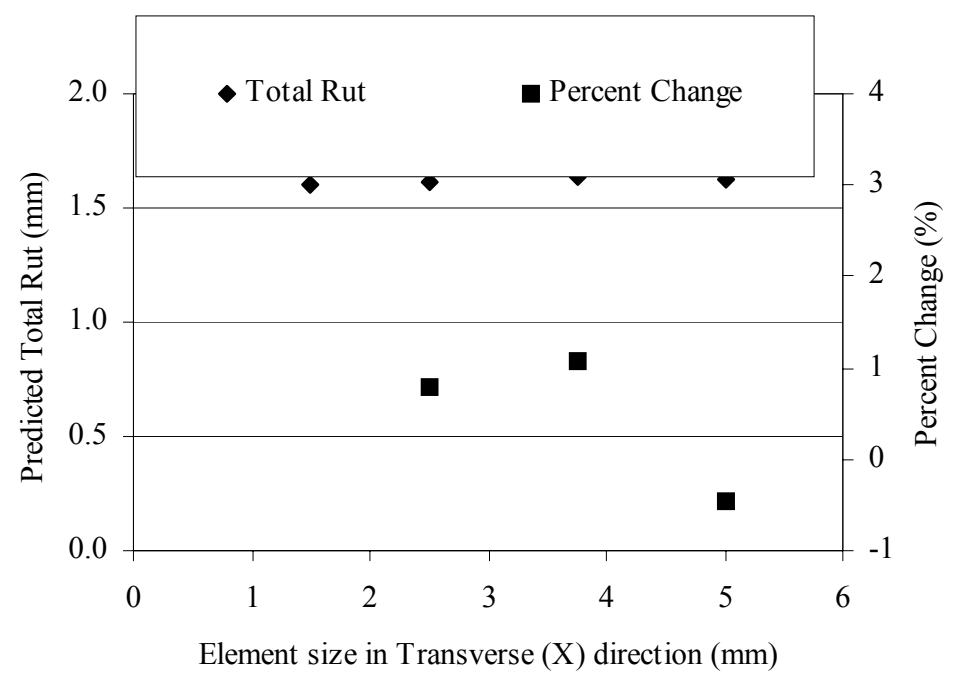

Figure 7-37 Effect of Transverse Direction Element Size (PURWheel)

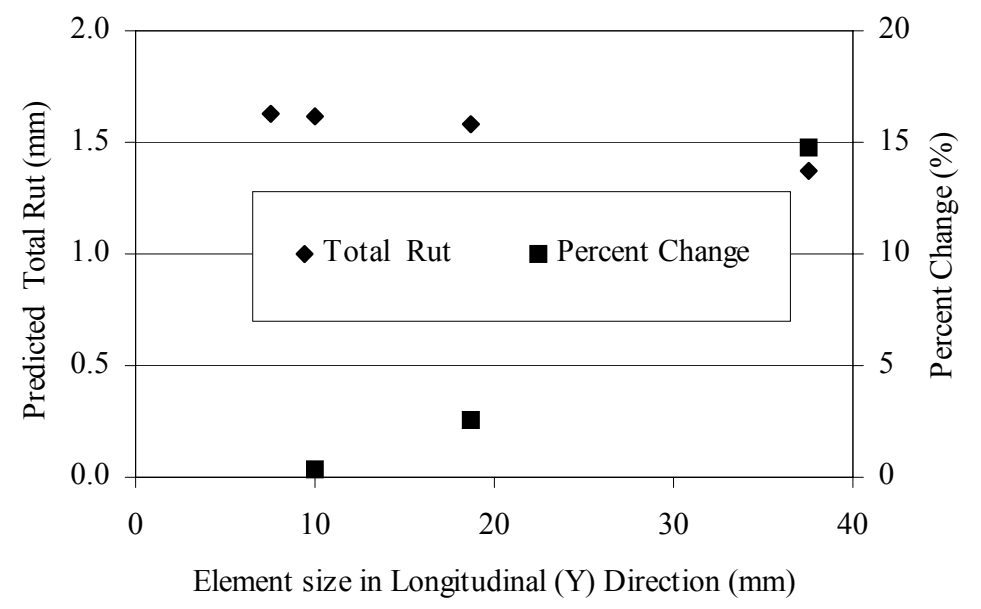

Figure 7-38 Effect of Longitudinal Direction Element Size (PURWheel) 


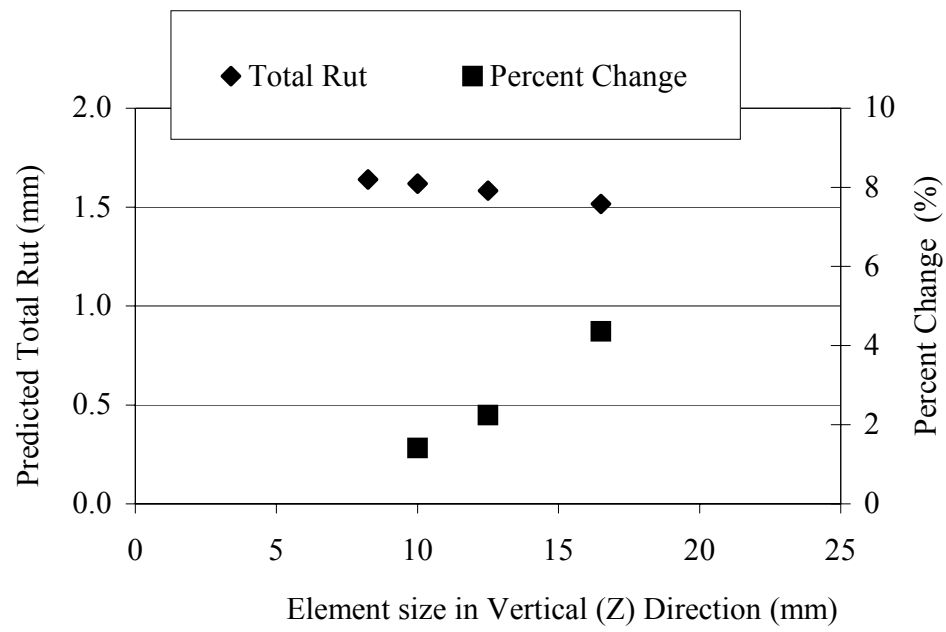

Figure 7-39 Effect of Vertical Direction Element Size (PURWheel)
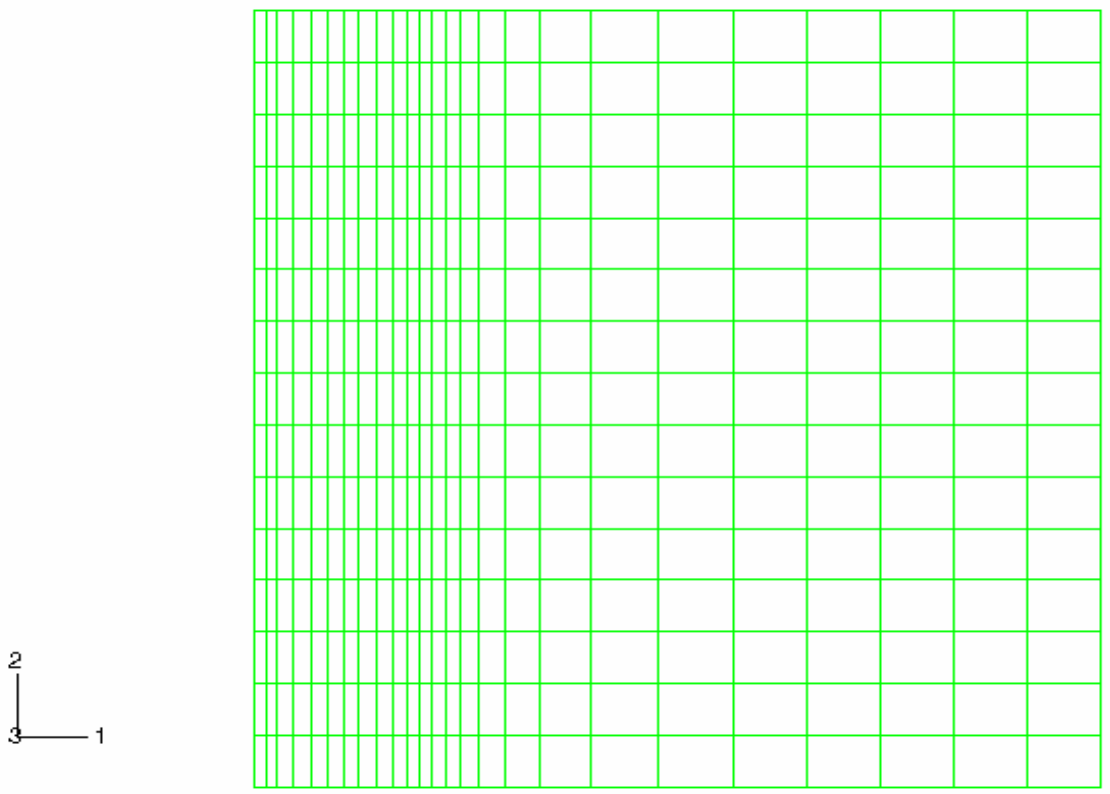

Figure 7-40 Plan View of the PURWheel Mesh 


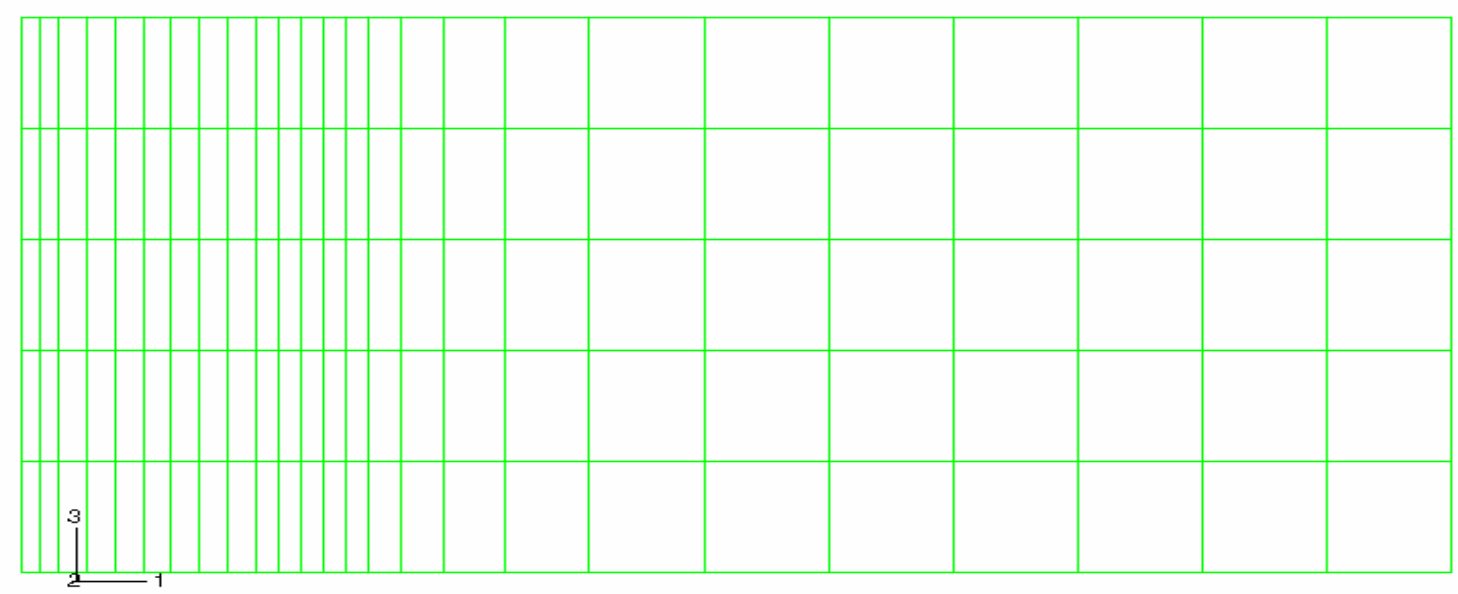

Figure 7-41 Elevation View of the PURWheel Mesh

\subsection{Sensitivity Studies}

The sensitivity of the PURWheel, APT, and test track FEM models with respect to the input variables was conducted. The input variables investigated were the creep model parameters $\mathrm{A}$ and $\mathrm{m}$.

\subsubsection{Effect of Creep Model Parameters}

The creep material model was defined using three material constants $\mathrm{A}, \mathrm{m}$, and $\mathrm{n}$. The rutting performance of the HMA mixtures was compared using calculated material constants A and $m$ while the n parameter was held constant. Figures 7-42 through 7-44 show the responses of the test track, the APT, and the PURWheel models to changes in parameter A. As A increases, the predicted rutting for all devices also increases.

Figures 7-45 through 7-47 show the variations in the rut depth as a function of the creep model parameter $m$ for the test track, the APT, and the PURWheel models. Again, the 
predicted rutting increases as $m$ increases. However, the NCAT test track, the APT and the PURWheel data show that at lower values of $m$ typically determined in this study (0.9 to -0.7$)$, the predicted total rut depth is less sensitive to $m$ than at higher values of $m$.

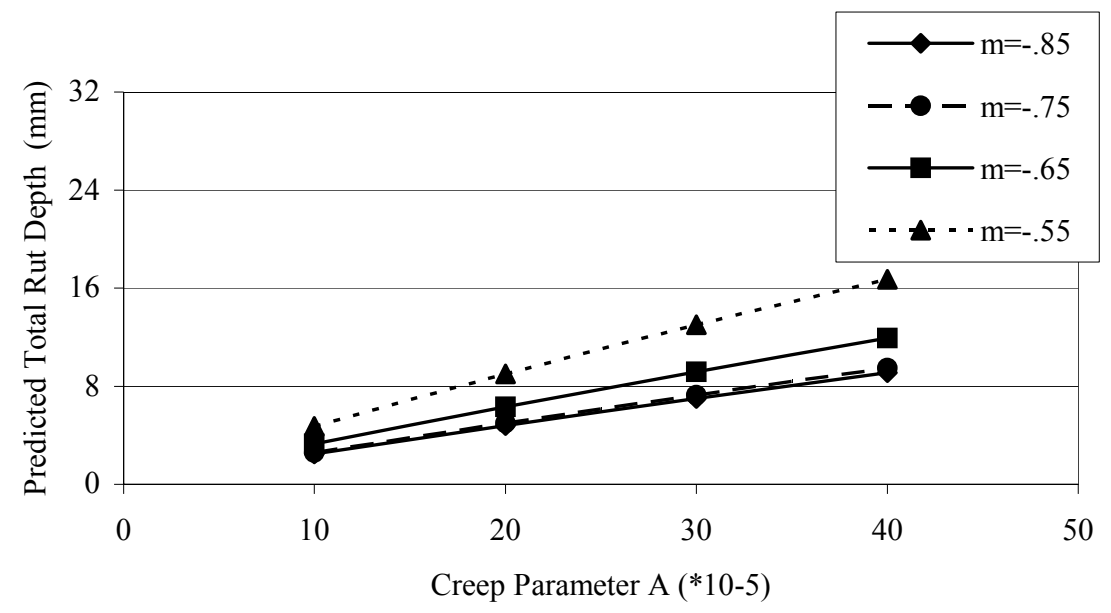

Figure 7-42 Effect of Creep Parameter A on NCAT Test Track Rutting (FEM)

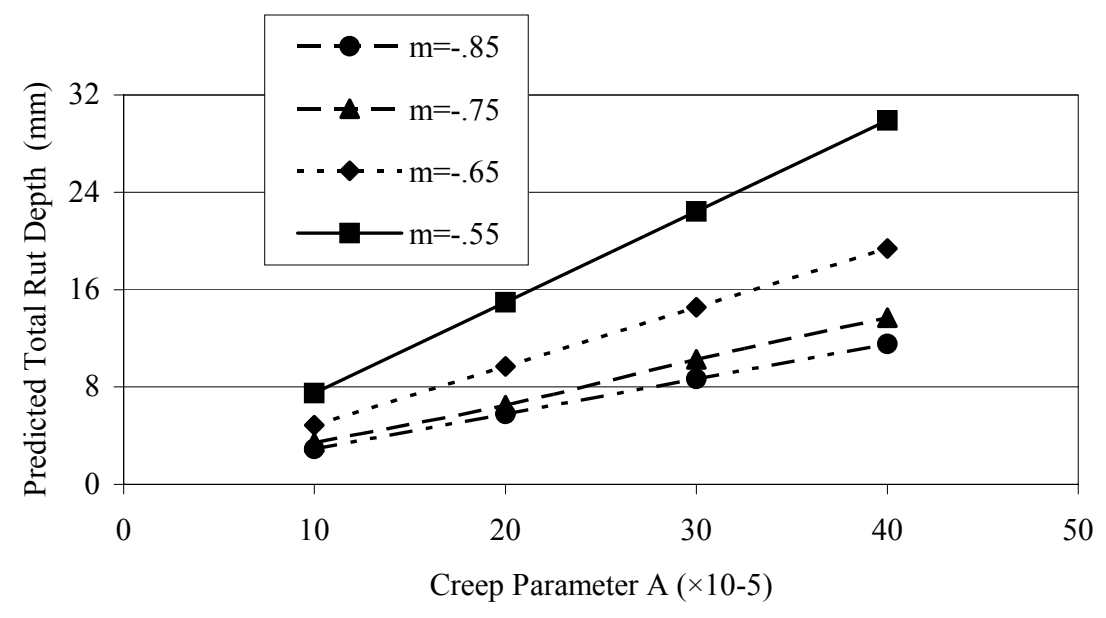

Figure 7-43 Effect of Creep Parameter A on APT Rutting (FEM) 


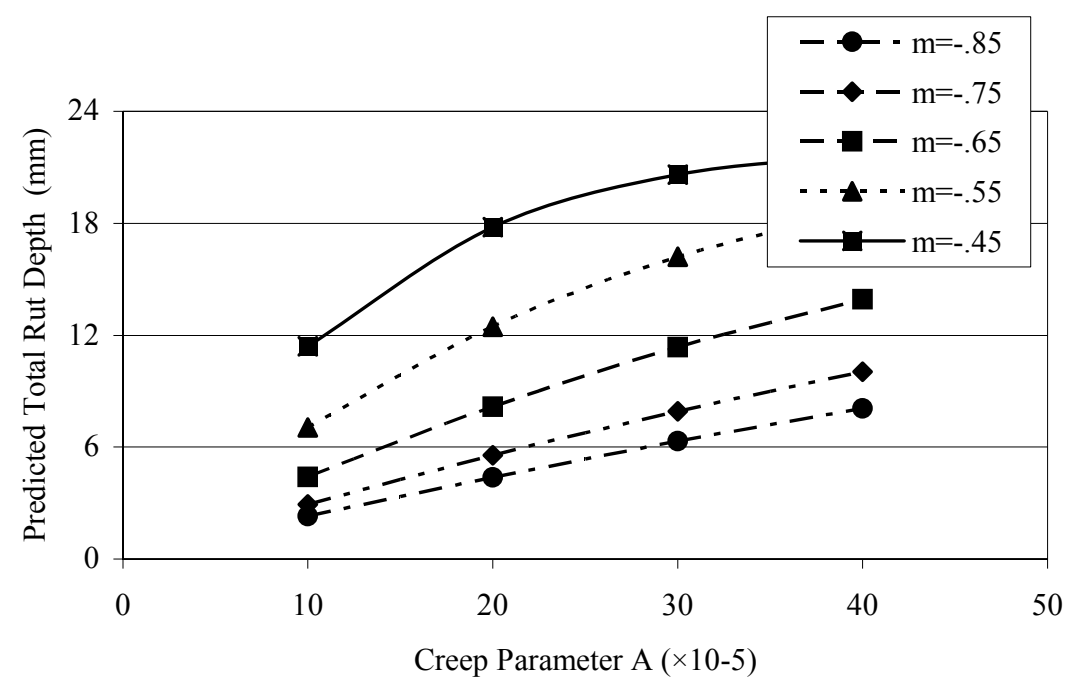

Figure 7-44 Effect of Creep Parameter A on PURWheel Rutting (FEM)

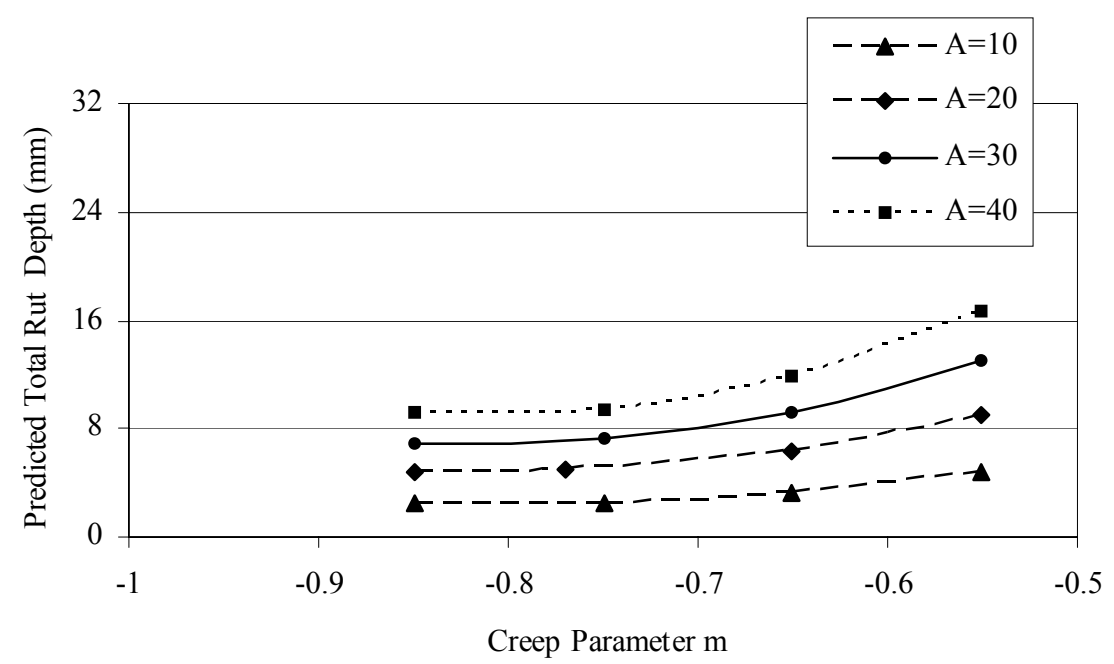

Figure 7-45 Effect of Creep Parameter m on NCAT Test Track Rutting (FEM) 


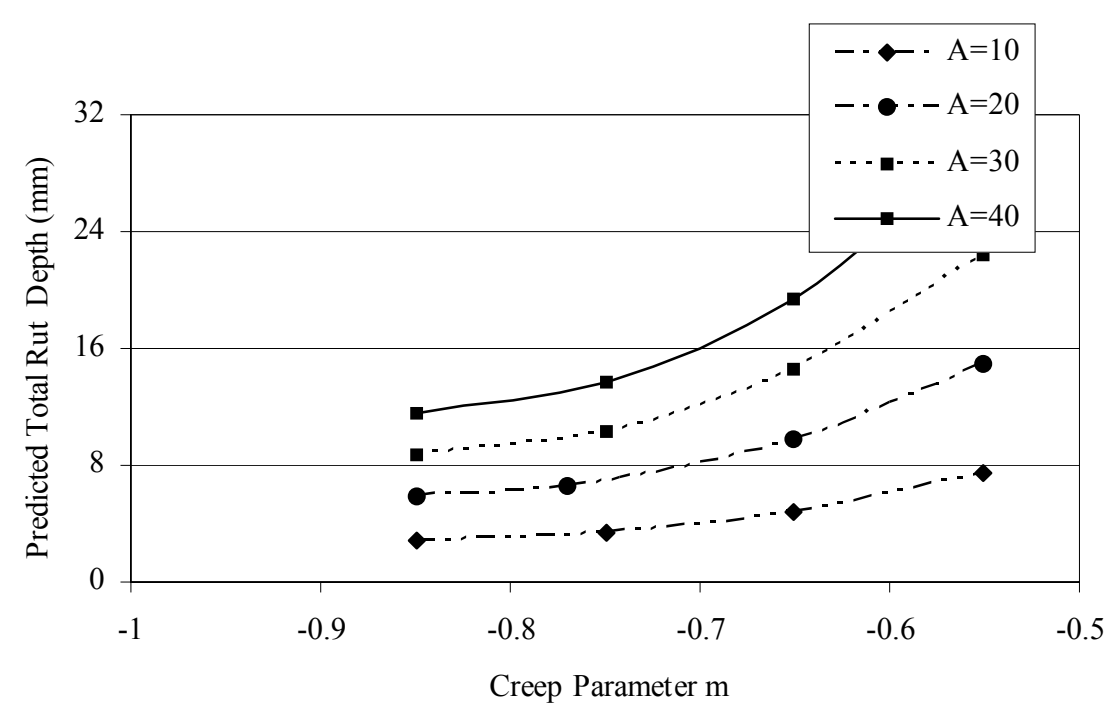

Figure 7-46 Effect of Creep Parameter m on APT Rutting (FEM)

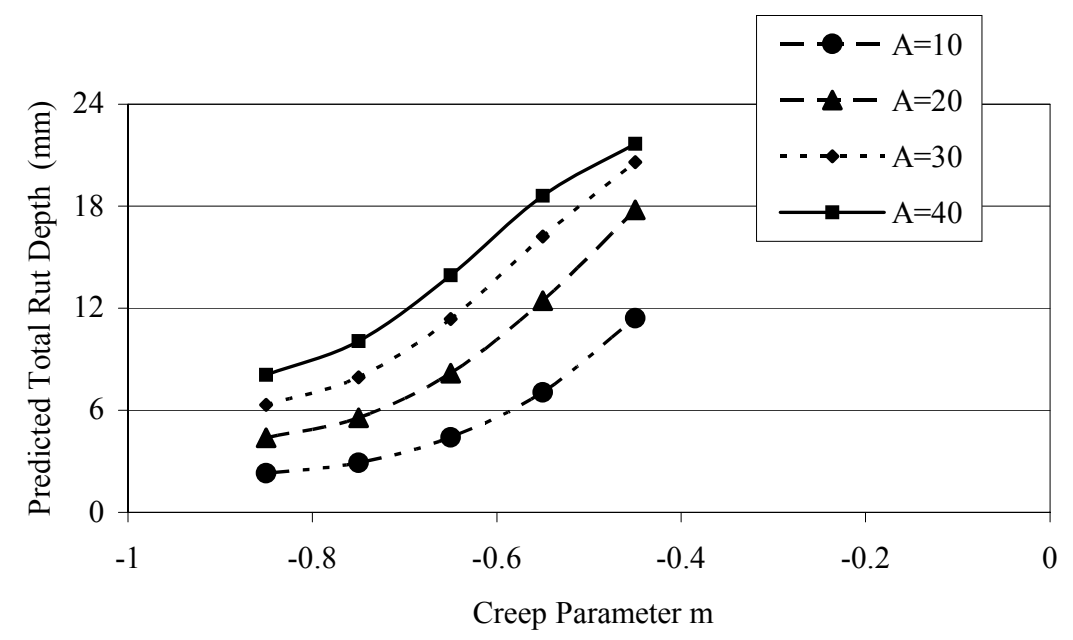

Figure 7-47 Effect of Creep Parameter m on PURWheel Rutting (FEM) 


\section{$\underline{7.10 \text { Determination of Creep Material Parameters }}$}

As discussed previously, an HMA mixture can be characterized using a creep model that is defined using three material constants, $\mathrm{A}, \mathrm{m}$, and $\mathrm{n}$. According to the discussions presented, a best fit procedure was used in conjunction with the rutting data to estimate the $\mathrm{A}$ and $\mathrm{m}$ material constants while the $\mathrm{n}$ parameter was fixed at 0.8 for each of the HMA mixtures tested. This was done by varying A and $m$ in the FEM model, until a reasonable match was observed between the measured and predicted rutting data. The difference was considered reasonable when the error between the two was within 10 to 15 percent. Generally, rutting development occurs at a faster rate for the first few hundred cycles (initial densification) followed by an approximate steady state. Figure 748 shows the typical APT rutting development using a simple regression model. The slope of the straight line is the rutting rate while the intercept can be related to the initial densification.

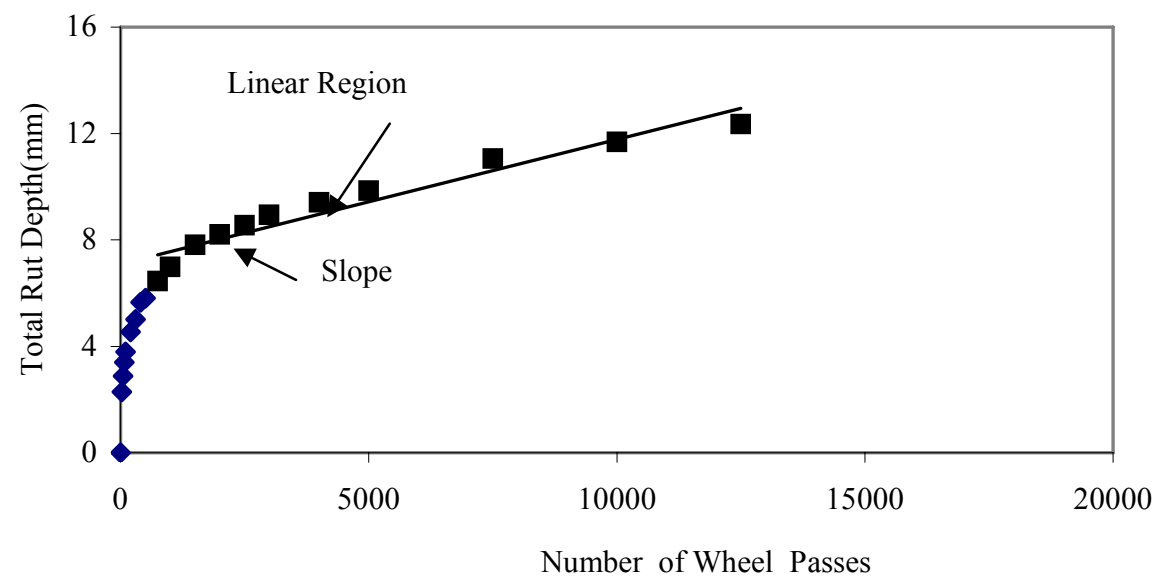

Figure 7-48 APT Rutting Development 


\subsubsection{Determination of NCAT Test Track Creep Parameters}

Materials creep parameters were determined for the test track so they could be compared to those obtained from the APT and PURWheel data. In order to incorporate the environmental temperature effects into the track modeling, the data were divided into seven different regions as previously explained. It was assumed that within a given region, the temperature conditions could be approximated as a constant. For such a condition, the rutting development can be approximated using a steady state, linear relationship as shown in Figure 7-48.

The test track rutting data indicated that rutting develops at a much faster rate for the first few thousand ESAL applications (Region I in Figure 7-14) and that the rate of rutting decreases as more traffic is applied. The data generated from the finite element model shows a similar trend with a higher rutting rate for the initial traffic loading and a decrease in rutting rate as additional traffic is applied. Also, the temperature at the test track was well below 40C (104F) during this time period, indicating that the initial densification occurs even when the temperatures are lower than required to produce rutting.

The determination of parameters for Regions II through VII of the test track had to account for the higher rutting rate that occurs for first few thousand ESAL. This adjustment procedure is shown in Figure 7-49. On each FEM computer run, the origination point of the FEM-generated data was shifted to a point above and to the right of the actual origin to a point where the rutting development occurs at a steady rate (constant rate of rutting). This adjustment is based on the fact that at constant 
temperatures, rutting development can be approximated by a linear relationship, as observed for the APT and the PURWheel data (Figure 7-48).

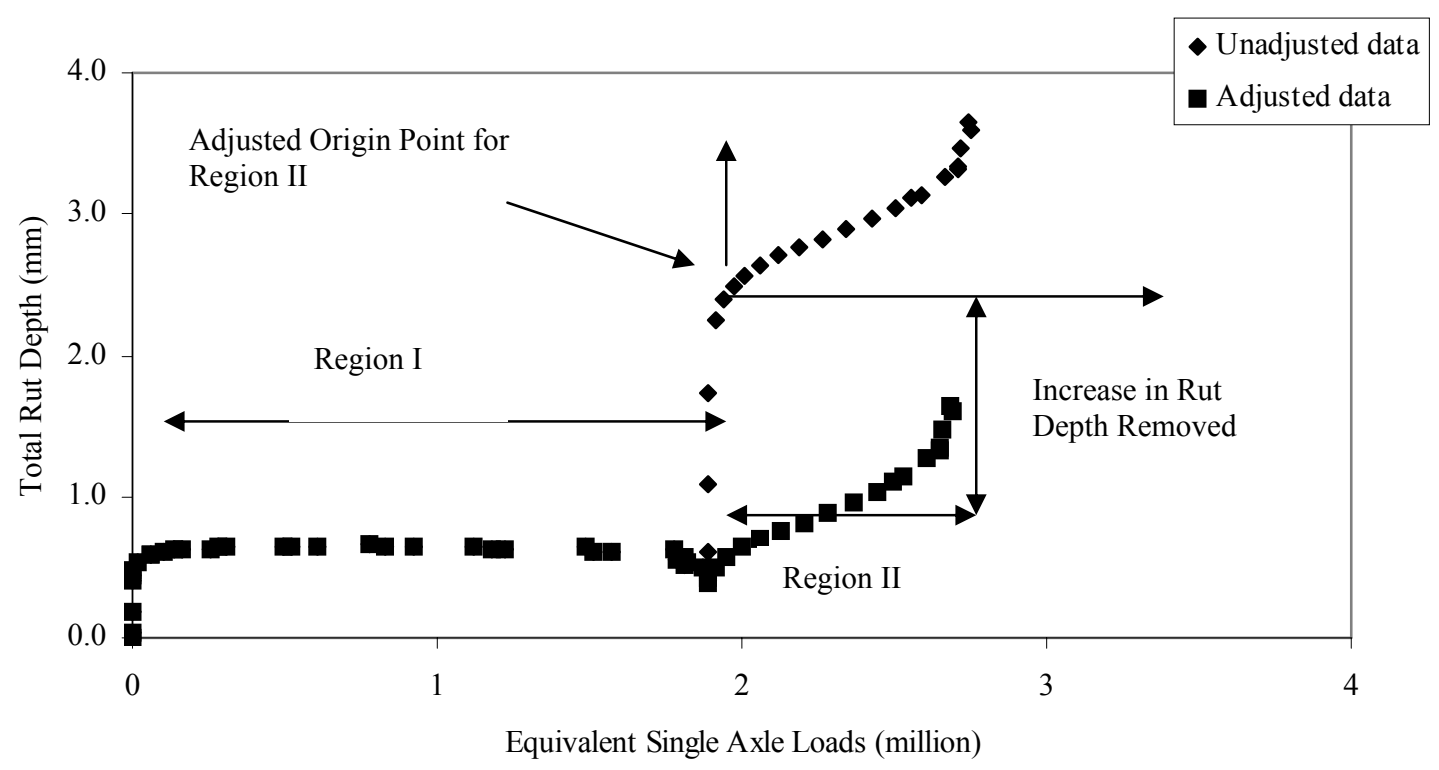

Figure 7-49 FEM Data Adjustment

Table 7-1 Creep Parameters of Test Track Mixtures (Region III)

\begin{tabular}{|c|c|c|c|}
\hline Section & $\mathrm{A}\left(10^{-5}\right)$ & $\mathrm{M}$ & $\mathrm{n}$ \\
\hline $\mathrm{N} 1$ & 9.0 & -0.860 & 0.8 \\
\hline $\mathrm{N} 2$ & 6.8 & -0.820 & 0.8 \\
\hline N3 & 20.0 & -0.800 & 0.8 \\
\hline N4 & 17.5 & -0.793 & 0.8 \\
\hline N5 & 15.0 & -0.850 & 0.8 \\
\hline N6 & 11.5 & -0.860 & 0.8 \\
\hline
\end{tabular}

The total rut depth was used in determining the parameters for the test track rutting. Table 7-1 shows the estimated creep parameters for each HMA mixture for Region III. The remaining data is given in Tables A-1 through A-4. Sections N7, N8, N9 and N10 were not used because actual rutting was minimal. Figure 7-50 shows the 
measured and predicted rut developments for the N4 section of the test track. Figures A-1 through A-5 show the data for the other test track mixtures.

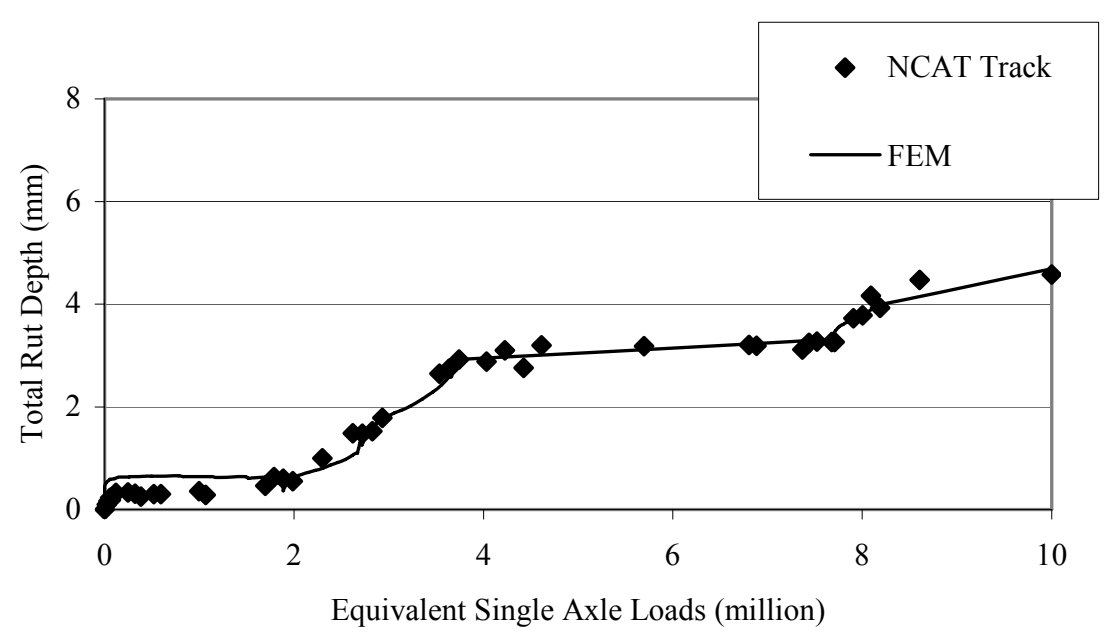

Figure 7-50 Rut Development in the N4 Test Track Section

\subsubsection{Determination of APT Creep Parameters}

The total rut depth development in the APT can be approximated by a linear relationship after the first few hundred passes. Any number of passes in this linear region can be used for the creep parameters determination. Accordingly, the determination of APT creep parameters was based on 10,000 wheel passes. In order to compare measured and predicted rut depth using estimated parameters, the last 2500 passes were not included. To improve consistency, the same number of wheel passes was selected and used with the PURWheel data as well.

For the APT, experimental data includes an uplift component and the compactive rut depth as well as the combination of the two, the total rut depth. However, the 
PURWheel test provides compactive rut data only. To be able to compare and contrast the APT and PURWheel results, the compactive rut depth was used for both the PURWheel and the APT. Table 7-2 presents the creep material property data for each APT mixture tested at 50C $(122 \mathrm{~F})$. Figure 7-51 shows the best fit curve used to determine the parameters of APT section N3 while plots of the remaining APT mixtures are shown in Figures A-6 through A-12. Table 7-3 shows the material data for APT mixtures tested at 35C (95F); only sections N1, N3, and N4 were tested in the APT at 35C (95F). Figures A-13 through A-15 are plots of the best-fit curves for these APT mixtures.

Table 7-2 Creep Parameters of APT Mixtures (50C)

\begin{tabular}{|c|c|c|c|}
\hline Section & $\mathrm{A}\left(10^{-5}\right)$ & $\mathrm{m}$ & $\mathrm{N}$ \\
\hline $\mathrm{N} 1$ & 16.5 & -0.860 & 0.8 \\
\hline N2 & 20.0 & -0.855 & 0.8 \\
\hline N3 & 35.0 & -0.800 & 0.8 \\
\hline N4 & 33.0 & -0.860 & 0.8 \\
\hline N5 & 35.5 & -0.860 & 0.8 \\
\hline N6 & 23.0 & -0.870 & 0.8 \\
\hline N9 & 35.0 & -0.860 & 0.8 \\
\hline N10 & 26.5 & -0.845 & 0.8 \\
\hline
\end{tabular}

Table 7-3 Creep Parameters of APT Mixtures (35C)

\begin{tabular}{|c|c|c|c|}
\hline Section & $\mathrm{A}\left(10^{-5}\right)$ & $\mathrm{m}$ & $\mathrm{N}$ \\
\hline $\mathrm{N} 1$ & 7.0 & -0.830 & 0.8 \\
\hline $\mathrm{N} 3$ & 21.0 & -0.810 & 0.8 \\
\hline $\mathrm{N} 4$ & 21.0 & -0.700 & 0.8 \\
\hline
\end{tabular}




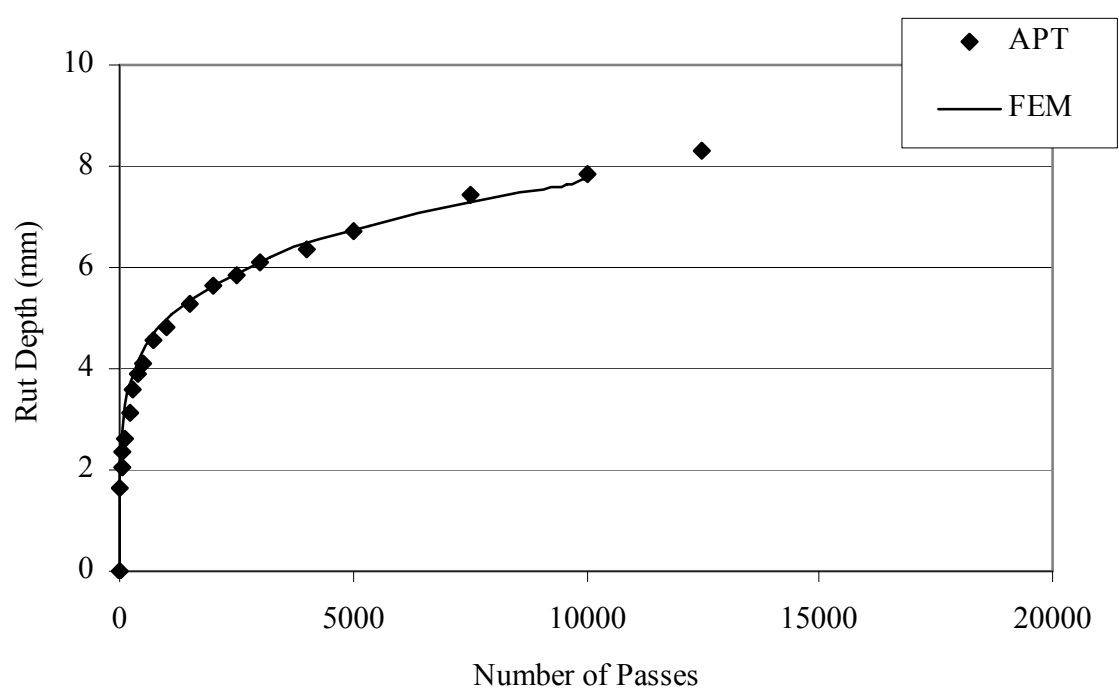

Figure 7-51 Parameter Determination for APT Section N3

\subsubsection{Determination of PURWheel Creep Parameters}

Figure 7-52 shows the best-fit determination for the N3 mixture when subjected to PURWheel testing. Plots for the other track mixtures are shown in Figures A-16 through A-22. Table 7-4 shows the creep parameters calculated for each HMA mixture. These PURWheel tests were conducted using specimens sawn from the test track. Slab specimens from Sections N9 and N10 were not tested in the PURWheel since they were too thick to be accommodated in the PURWheel sample-mounting box. 


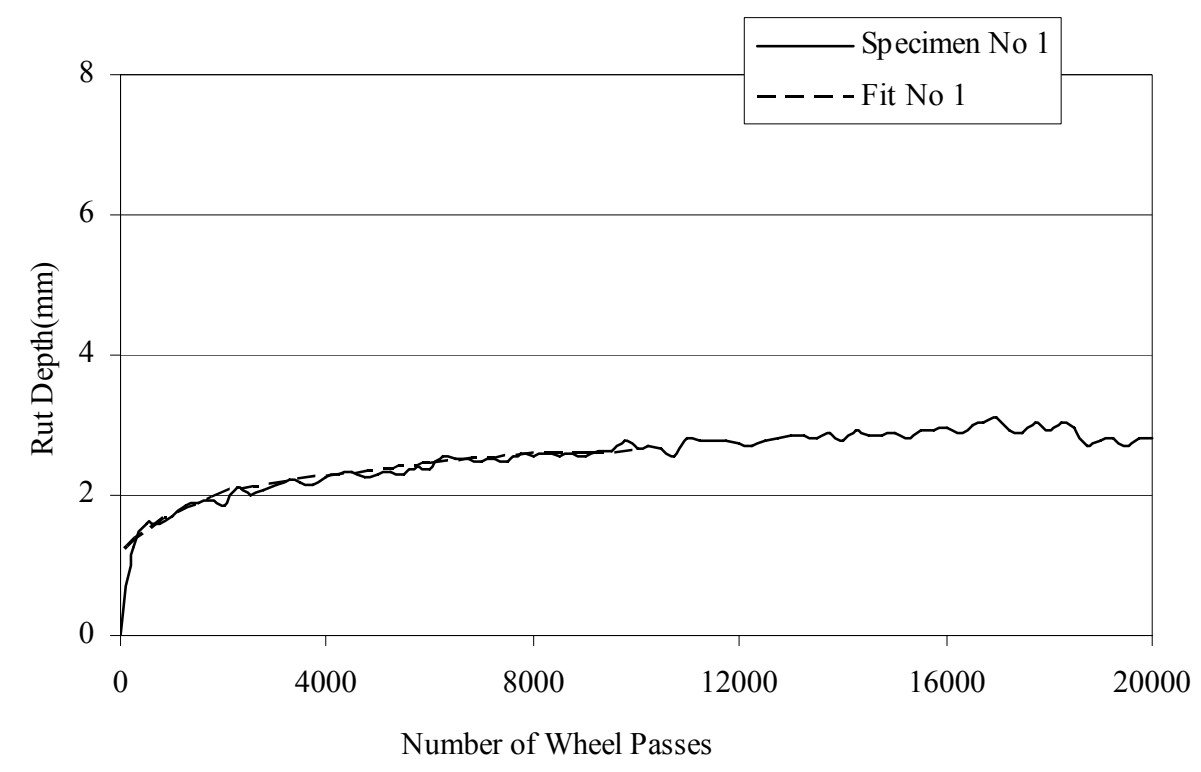

Figure 7-52 Parameter Determination for PURWheel Section N3 (Track Slabs)

Table 7-4 Creep Parameters of PURWheel Mixtures (Track Slabs)

\begin{tabular}{|c|c|c|c|}
\hline Section & $\mathrm{A}\left(10^{-5}\right)$ & $\mathrm{m}$ & $\mathrm{n}$ \\
\hline $\mathrm{N} 1$ & 11.5 & -0.860 & 0.8 \\
\hline $\mathrm{N} 2$ & 13.5 & -0.820 & 0.8 \\
\hline N3 & 20.0 & -0.800 & 0.8 \\
\hline N4 & 14.5 & -0.793 & 0.8 \\
\hline N5 & 19.0 & -0.850 & 0.8 \\
\hline N6 & 15.0 & -0.840 & 0.8 \\
\hline N7 & 14.0 & -0.870 & 0.8 \\
\hline N8 & 13.5 & -0.840 & 0.8 \\
\hline
\end{tabular}

Figures A-23 through A-29 show the plots for slab specimens taken from the APT prior to traffic application and tested in the PURWheel. Table A-5 summarizes creep parameters. Generally two specimens were tested for a given HMA mixture. Parameter calculation was based on the compactive rut depth instead of total rut depth, since the PURWheel rutting history data was available only for compactive rut depth. For each 
specimen, parameter determination was performed individually and the average creep parameters were reported. This is to account for the wheel velocity difference that was noted between the right and the left wheel and hence the total loading time difference. No rutting history data was available for Section N3 and consequently no creep parameters were determined.

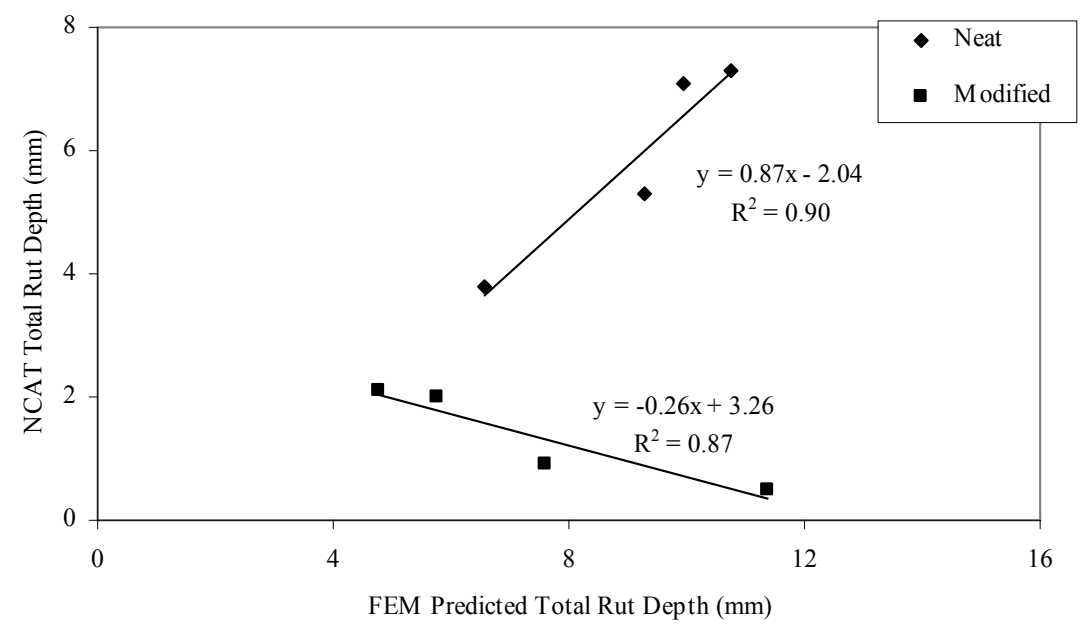

Figure 7-53 Comparison of Measured and Predicted Track Rut Depths (APT Material Properties)

\subsubsection{Rutting Relationships using Determined Creep Parameters}

Figure 7-53 shows a comparison between the measured rut depths at the test track after 10 million ESAL applications and the rut depths predicted by the FEM track model using the APT material properties with the same amount of ESAL applications. An excellent correlation was achieved for the mixtures containing neat binders $\left(\mathrm{R}^{2}=0.9\right)$. Figure 7-53 also shows that the modified binder mixtures exhibited a strong relationship $\left(\mathrm{R}^{2}=0.87\right)$. However, the trend is reversed in that the test track mixtures with the least 
rutting exhibited the higher rutting in the FEM model. This same trend was seen in the direct comparison of the test track and APT rutting data (see Figure 6.26) and is thought to be a result of the differences in initial densities. However, even with this reverse trend, it does appear that the FEM approach does have merit for predicting test track rutting using the APT rutting data.

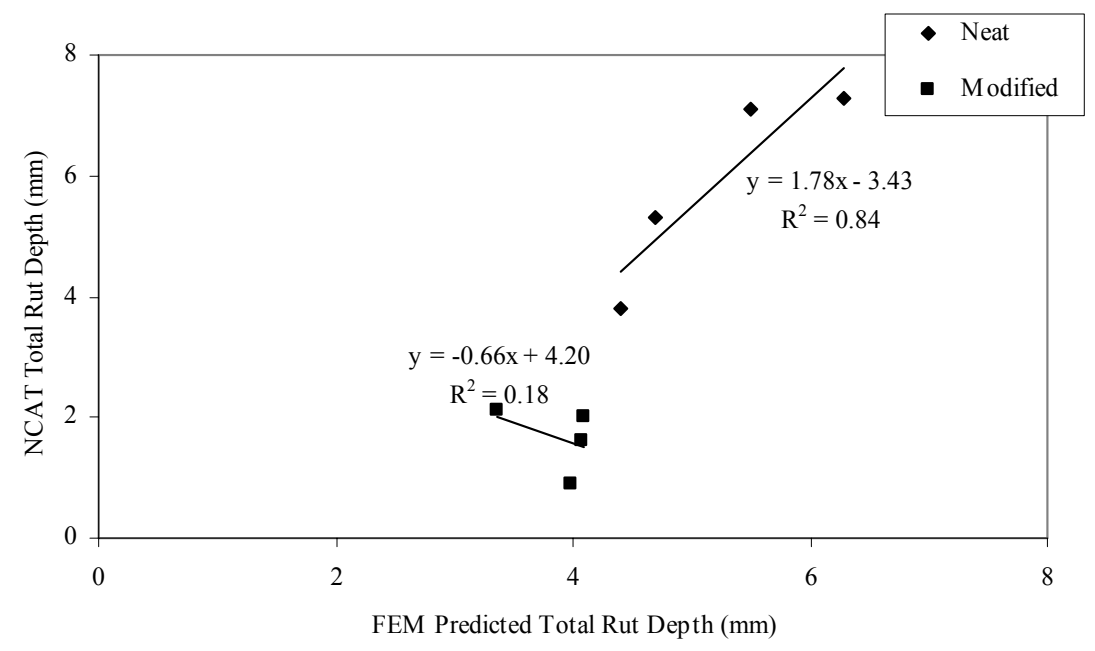

Figure 7-54 Comparison of Measured and Predicted Track Rut Depth (PURWheel Material Properties)

The analogous plot for the PURWheel data is shown in Figure 7-54. Here the PURWheel data obtained using slab specimens taken from the test track was used to determine the material properties of the mixtures. These parameters were then used in the FEM track model to predict test track rutting after 10 million ESAL applications. The neat binder mixtures show an excellent correlation $\left(\mathrm{R}^{2}=0.84\right)$ while the modified binder mixtures do not show a clear trend. The direct comparison between the test track and PURWheel rutting data likewise showed no clear trend (see Figure 6.27). It appears that 
the PURWheel may not be sensitive enough for mixtures that exhibit very little rutting in the field. However, with some further work, it may be possible to use PURWheel data and FEM to predict in-service rutting.

\subsubsection{Densification Effects on the Rutting Performance (FEM)}

The amount of rutting that developed during the first (Region IV) and second (Region VII) winters was slight because the average temperatures of the test track were well below the temperatures necessary for rutting to occur. Since the developed rutting is so small during these periods, they were not included in the FEM rutting development model. However, some amount of rutting was developed during the initial consolidation period even when the temperatures were lower than those normally required for rutting development.

The material properties data from Region III (first summer, high temperature) was compared with that of the Region VI (second summer, high temperature). The results suggest that the A parameter of Region VI is lower than that of Region III as indicated in Figure 7-55 (most of the data points are located below the line of equality). This may confirm the effect of densification on rutting performance. During the first summer, when the mixtures were subjected to higher temperatures, they were more susceptible to rutting. When the same mixtures were subjected to high temperatures a second time, less rutting occur. This is likely due to the initial densification during the first summer; the mixtures had less rutting potential the second summer. It is also important to note that the m parameter in Region VI is higher for some mixtures than for Region III as shown in 
Figure 7-56. This is probably due to the fact that the second summer was comparatively warmer than the first.

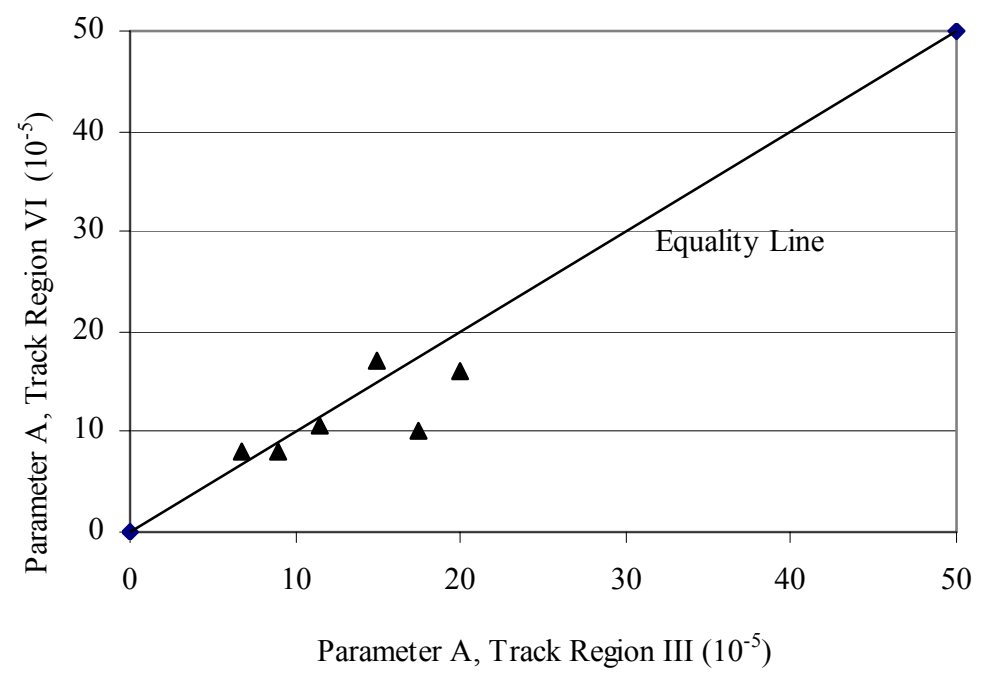

Figure 7-55 Comparison of Rutting Performance, Regions III and VI

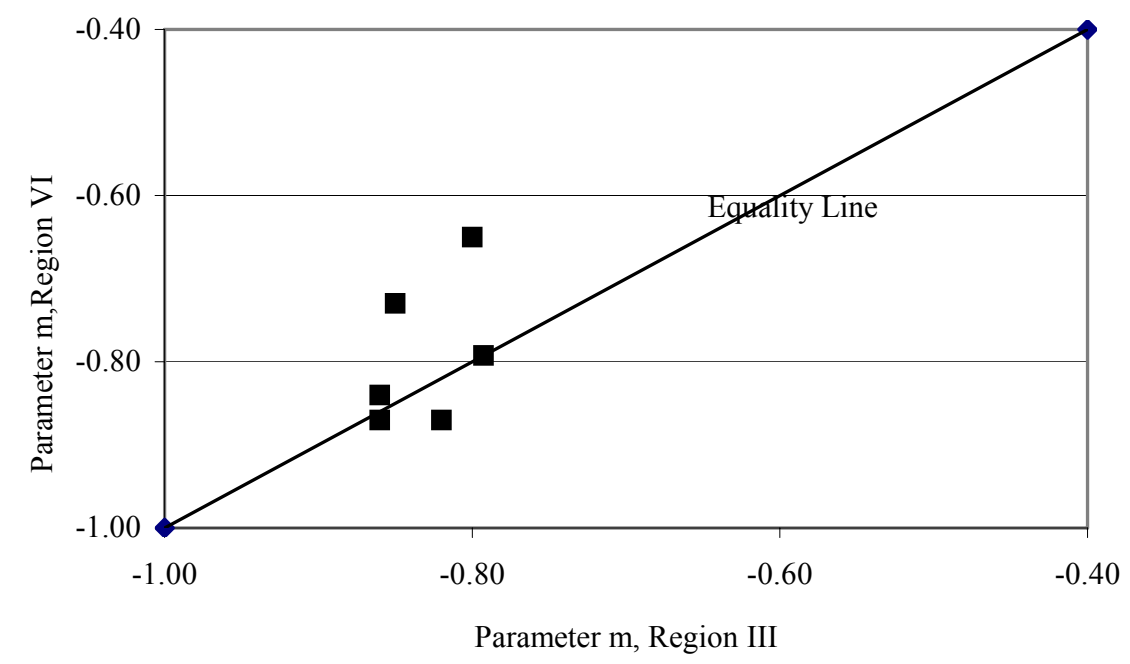

Figure 7-56 Comparison of Rutting Performance, Regions III and VI 


\subsection{Temperature Effects on Rutting Performance (FEM)}

The rutting performance of a given HMA mixture is a high temperature phenomenon and attempts were made to compare rutting performance at different temperatures in the APT and/or test track. Three HMA mixtures (N1, N2, and N3) were tested at both 35 and 50C ( 95 and 122F) in the APT. This data provided a basis to study temperature effects in the APT. Table 7- 3 presents the material property data for the mixtures tested at $35 \mathrm{C}$ in the APT.

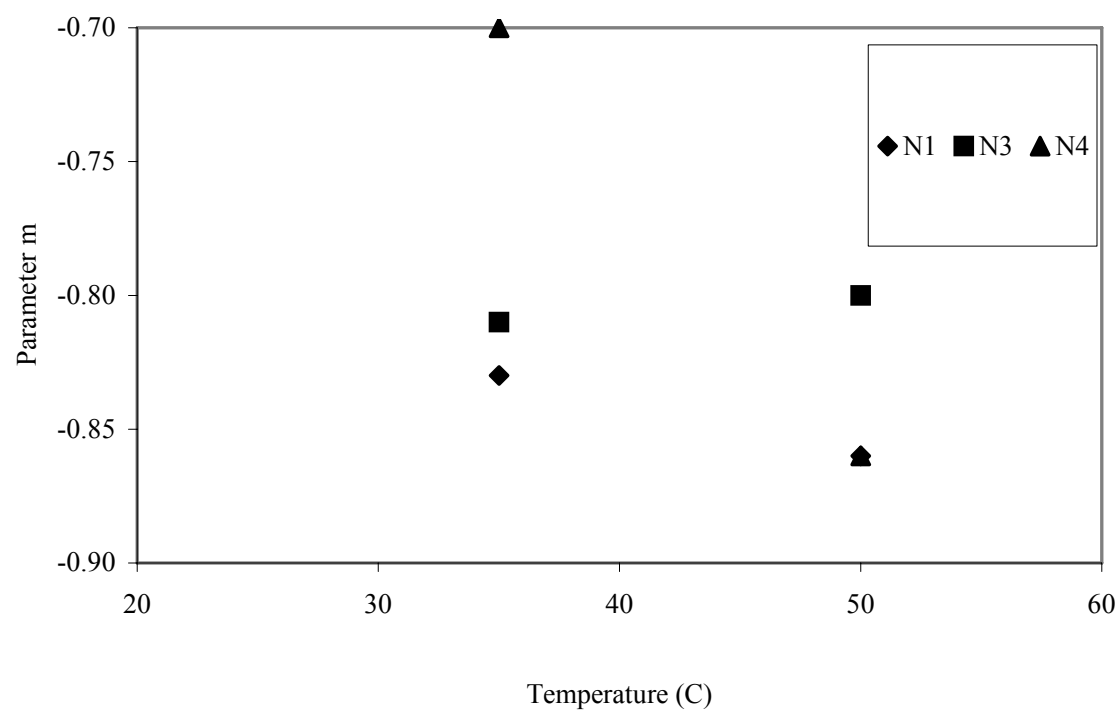

Figure 7-57 Effect of Temperature on Parameter m (APT)

Figure 7-57 shows the variation of the m parameter with temperature for the APT data. No clear trend is evident. Figure 7-58 is a plot of the variation of the A parameter (which is more sensitive to rutting for the typical values of $m$ determined on this study) with the temperature. The figure shows that increasing temperature also increases the value of A. However, the rate of variation is different. In other words, the temperature 
sensitivity of each HMA mixture to is not equal. Comparing the slopes of the curves presented in Figure 7-58 indicates that the modified binder mixture (N1) shows minimum change (through minimal change in A) while neat binder mixture (N3) exhibited maximum change in parameter $\mathrm{A}$.

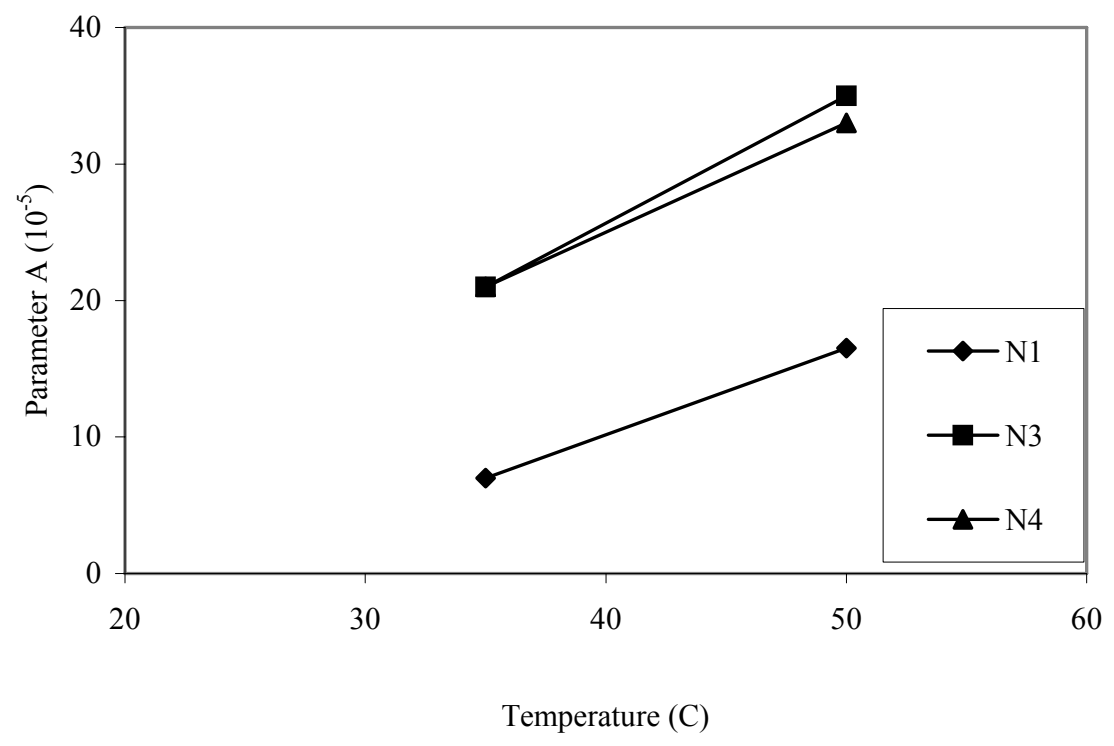

Figure 7-58 Effect of Temperature on Parameter A (APT)

Figure 7-59 shows how the m parameter varies with initial pavement density. As expected, an increase in the initial density results in a lower $\mathrm{m}$ value. However, a better correlation was observed for tests conducted at $50 \mathrm{C}\left(\mathrm{R}^{2}=0.54\right)$ than for tests conducted at $35 \mathrm{C}\left(\mathrm{R}^{2}=0.13\right)$. This indicates that the initial density effects on parameter $\mathrm{m}$ are more pronounced at higher temperatures.

The variation of parameter A as a function of initial pavement density is shown in Figure 7-60. A stronger relationship exists between parameter A and initial density as 
compared to the parameter $\mathrm{m}$, regardless of the temperature. This indicates that parameter $\mathrm{A}$ is more related to initial density than is the $\mathrm{m}$ parameter.

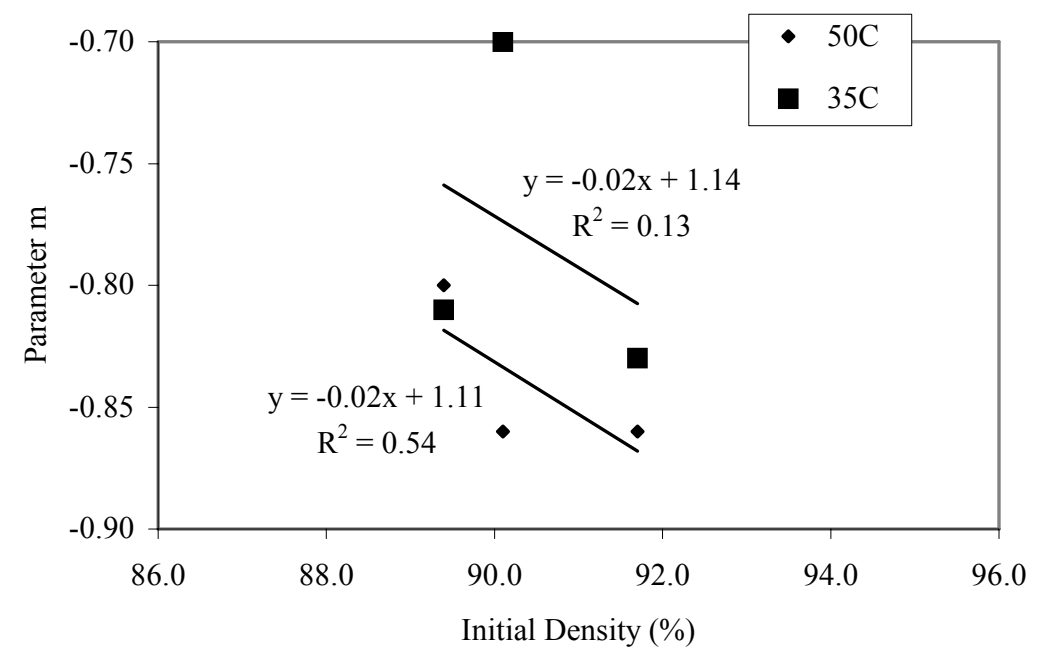

Figure 7-59 Effect of Initial Density on Parameter m at Different Temperatures (APT)

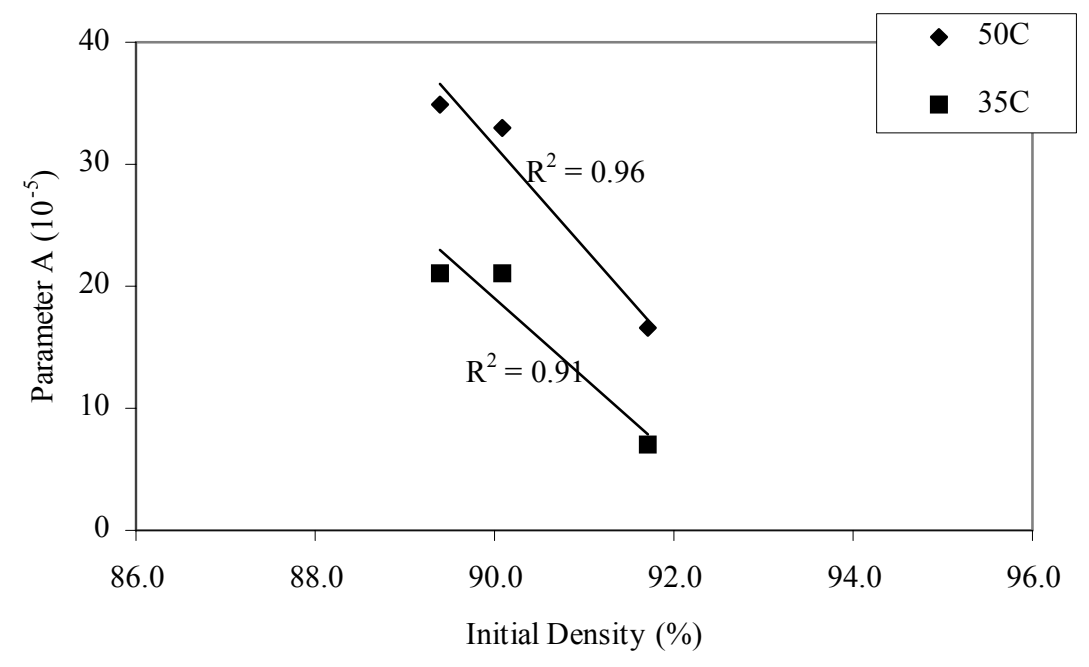

Figure 7-60 Effect of Initial Density on Parameter A at Different Temperatures (APT) 
The test track rutting was divided into seven different regions to account for the temperature effects on rutting performance. This approach presented the opportunity to compare the creep material parameters obtained at 40 (Region II) and 50C (Region III) (104 and 122F). The creep parameters were not calculated for some mixtures since very little rutting occurred during the time period of Regions II and III. Figure 7-61 shows the effect of the temperature on the m parameter while Figure 7-62 shows the effect on parameter A. As expected, increasing the temperature increases both parameters; however, the rate of change was not equal. For the m parameter, Section N3 exhibited the highest rate of change with temperature; Section N5 exhibited the highest rate of change with temperature for parameter A. Sections N3 and N5 were the neat binder mixtures with optimum plus one-half percent binder content. N3 was fine-graded while N5 was coarse-graded. The results compare well with the expected results; mixtures containing neat binders at higher than optimum binder contents are more sensitive to temperature changes.

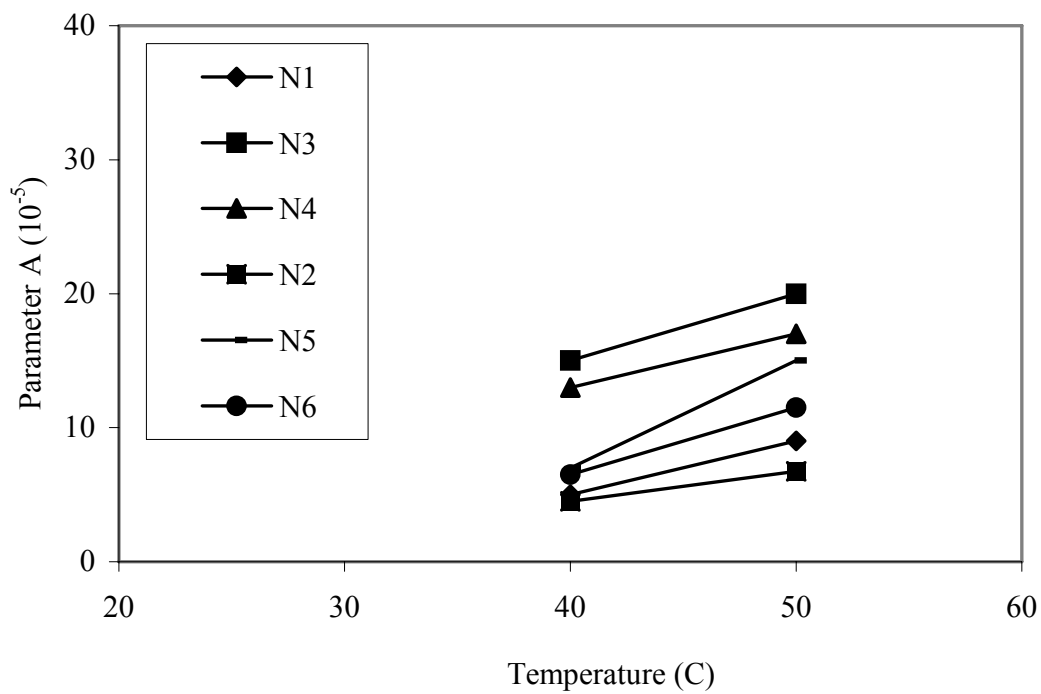

Figure 7-61 Variation of Parameter A with Temperature (Track, $1^{\text {st }}$ Summer) 


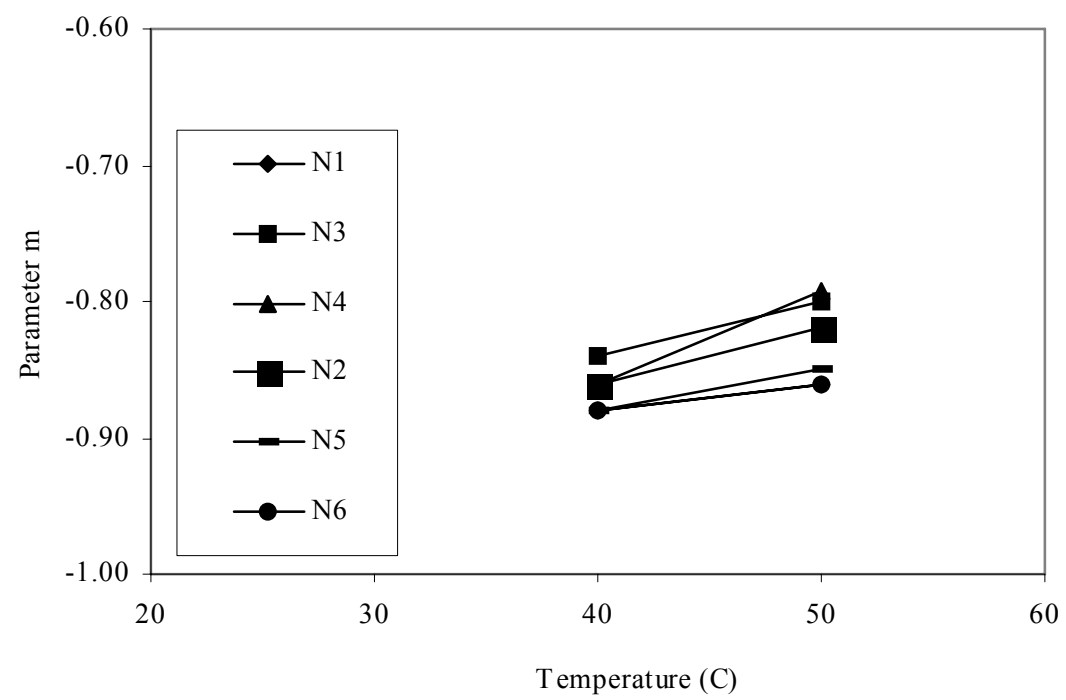

Figure 7-62 Variation of Parameter $\mathrm{m}$ with Temperatures (Track, $1^{\text {st }}$ Summer) 


\section{CHAPTER 8 CONCLUSIONS AND RECOMMENDATIONS}

\section{$\underline{8.1 \text { Conclusions }}$}

The INDOT adopted the Superpave mixture design method and specifications with the expectation of HMA performance benefits. However, since no long-term (20 years) performance data then existed, INDOT decided to participate in the NCAT fullscale test track as a way to validate the Superpave design method and specifications. Additionally, the INDOT was interested in establishing rutting performance relationships between the APT, the PURWheel, and in-service HMA pavements as represented by the NCAT test track.

The objectives of the project were completed using a coordinated effort between INDOT, ALDOT, and the FHWA. This test plan placed ten HMA mixtures on the NCAT test track and included three binder types (one neat and two modified), two gradations (fine- and coarse-graded), and two binder contents (optimum and optimum plus one-half percent). Ten million ESAL were applied to these test sections over a two-year period. In addition, eight of the ten track mixtures were duplicated in the APT using the same materials as used for the track mixtures. Slab specimens were cut from both the APT and the test track before the application of traffic and these were tested in the PURWheel. 


\subsubsection{Superpave Mixture Design Method Validation}

The volumetric properties of each of the ten mixtures was computed and statistically related to HMA rutting performance after 10 million ESAL applications on the test track. The following conclusions are drawn from this effort:

1. The Superpave mixture design method and specifications provide wellperforming HMA mixtures with respect to permanent deformation. As with all HMA projects, quality materials should be used and sound construction practices followed;

2. For the Superpave designed mixtures in this study, the use of modified binders provided higher rutting resistance than did the unmodified binders. Further, the modified binders allowed for larger binder content variation without compromising rutting performance; and

3. The fine-graded Superpave mixtures used in this study showed good performance under the heavy traffic applications at the test track.

\subsubsection{Establishment of Rutting Performance Relationships}

Efforts to establish rutting performance relationships among the APT, PURWheel, and NCAT test track consisted of two approaches. The first directly related HMA mixture rutting performance from the PURWheel, APT, and test track. The second used finite element modeling to predict test track rutting based on PURWheel and APT test results. The direct relationship effort resulted in the following findings:

1. The direct rutting performance relationship between the APT and the PURWheel is very strong when the PURWheel specimens are taken directly 
from the APT test sections. The correlation is weaker when PURWheel specimens are laboratory prepared;

2. There is a fair correlation between the PURWheel and test track when PURWheel specimens are taken from the test track. Again, when laboratory prepared PURWheel specimens are used, the relationship is significantly weaker;

3. The APT and test track show a fair correlation in rutting performance. This correlation could likely be improved with better initial density control during APT test section construction;

4. Given the relationships established in this research, it should be possible for INDOT to place and test various HMA mixtures in the APT and/or PURWheel and use the results to predict in-service performance of the mixtures;

5. The PURWheel appears to be sensitive to the sample preparation (compaction) method. In order for the PURWheel test to better predict inservice performance, PURWheel test specimens must be prepared so as to duplicate the in-service compaction of HMA.

6. The APT appears to be less sensitive to binder content and type than is the test track; and

7. Duplication of in-service mixtures (including initial density) in the APT appears to be a critical element in performing accelerated testing.

The finite element approach for establishing relationships among the test track, APT, and PURWheel first determined creep material parameters using APT and 
PURWheel data and used these as inputs into a finite element model of the NCAT test track. The track rutting predicted by this approach was then compared to the actual rutting experienced at the test track. The results suggest the following:

1. NCAT test track rutting primarily occurred when the HMA surface course temperature reached approximately 50C (122F). Between 40 and 50C (104 and $122 \mathrm{~F}$ ) only a minimal amount of rutting developed. Below 40C (104) no rutting developed;

2. Using creep material parameters derived from PURWheel tests (specimens taken from the test track) as inputs in the test track FEM model resulted in reasonably close predictions of test track rutting for HMA mixtures containing neat binders. The predictions were not as accurate for HMA mixtures containing modified binders;

3. Using creep material parameters derived from the APT data as inputs in the track FEM model resulted in slight over predictions of test track rutting performance for both neat and modified binder mixtures. The over prediction is thought to be caused by the poor initial densities in some of the APT test sections; and

4. Finite element modeling techniques appear to be capable of predicting inservice rutting if all variables, such as temperatures and compaction, are properly accounted for in the modeling. 


\subsection{Recommendations}

Based on the study results that indicate both APT and the PURWheel data can be related to in-service HMA mixture performance, a dual methodology is proposed that makes use of both the APT and PURWheel. The first involves using the APT to test the stability of HMA mixtures that are being proposed for use in Indiana. Due to the cost of using the APT facility, this option is not seen as a daily test to determine whether any given mixture design will provide adequate performance, but rather the APT can be used in a research capacity to test new types of HMA mixtures and new mixture technology. For example, the APT could be used to quantify the benefits of a new mixture type thus giving INDOT the quantitative data needed to modify specifications and practices. In order to use the APT in this manner, the following should be completed:

1. Validate the rutting performance relationships between the APT and inservice mixtures over a wider range of materials;

2. Develop and codify a standard protocol for experimental design, construction methods, and testing in the APT; and

3. Consider building an additional APT facility in order to accommodate the amount of work that will be required.

The PURWheel also appears to be able to predict in-service rutting of HMA mixtures. However, it suffers from two deficiencies: there is only one PURWheel currently in operation and there is no company that manufactures them. These limitations make the daily use of the PURWheel problematic. If INODT desires to have a laboratory wheel tracking test that can be used for daily mixture design and field production testing, it is recommended that the PURWheel be used as a research tool to investigate the use of 
various other laboratory wheel tracking devices such as the Asphalt Pavement Analyzer and the Hamburg machine that are more suited to such operation. However, before such a scheme can be fully implemented, additional information is required. The following are recommended:

1. Develop a better method for the laboratory compaction of PURWheel samples. Such a method should produce test specimens having densities and aggregate orientations comparable to those found in in-service pavements; and

2. Validate the rutting performance relationships between the PURWheel and inservice mixtures over a wider range of materials.

Finally, it is recommended that INDOT consider further refinement of the finite element methodology completed in this research. The approach could use either APT or PURWheel data for predicting in-service rutting. APT or PURWheel testing would be performed 50C (122F) on HMA mixture specimens that duplicate initial in-service mixture properties. The material creep parameters determined from these tests would then be used in the finite element model to predict the in-service rutting performance for a given HMA mixture. Additionally, it may be possible to incorporate the fatigue testing results of the second NCAT test track experiment into the model. The rutting and fatigue results of the finite element modeling might then be used in conjunction with the pavement performance modeling currently used by the INDOT to determine the performance of any given HMA pavement in order to schedule needed maintenance activities in a timely fashion. 


\subsection{Implementation}

The research reported herein clearly demonstrates that the Superpave mixture design method and specifications do provide well-performing HMA mixtures when quality materials are used and sound construction practices followed. The following implementation item is suggested:

1. INDOT should fully adopt the Superpave mixture design method and specifications. Further, the INDOT should continue to monitor the performance of Superpave mixtures and make changes to the method and specifications as necessary.

Relationships among in-service pavements (as represented by the test track), APT, and PURWheel were developed in this research. The findings indicate that if APT and PURWheel specimens are correctly produced and compacted, good correlations between these and in-service pavements do exist. The following implementation items are recommended:

1. Establish a standard method for using the APT to test HMA mixture stability for new types of mixtures, mixture design approaches, and new mixture technologies; and

2. Consider completing a research program that uses the PURWheel to determine if another, more common laboratory wheel track test machine can be used for daily mixture design and field production testing. 


\section{REFERENCES}

ABAQUS/Standard Users’ Manual, Version 6.3, Hibbit, Karlson \& Soren, 2001

ABAQUS/Theory Manual, Version 6.3, Hibbit, Karlson \& Soren, 2001

ABAQUS/CAE, Standard Users’ Manual, Version 6.3, Hibbit, Karlson \& Soren, 2001

Archilla, A.R. and S. Madanat, "Development of pavement Rutting Model from Experimental Data,” Journal of Transportation Engineering, Vol.126, No 4, 2000, pp.291-299.

Badaruddin, S., and T.D. White, “Asphalt Mix Design and Performance,” Report No. FHWA/IN/JHRP-94/03, 1995.

Bonaquist, R.J. and W.S. Mogawer, "Analysis of Pavement Rutting Data from FHWA Pavement Testing Facility Superpave Validation Study,” Transportation Research Record 1590, Transportation Research Board, National Research Council, Washington, D.C., 1997.

Bonaquist, R., J. Sherwood, and K. Stuart, "Accelerated Pavement Testing at the Federal Highway Administration Pavement Testing Facility,” Journal of the Association of Asphalt Paving Technologists, Vol. 67, St. Paul, MN, 1998.

Bouldin, M.G., G. Row, J. Souse, and J.Sharmock, "Mix Rheology-a tool for predicting the high performance of hot mix asphalt," ,Journal of the Association of Asphalt Paving Technologists, Vol. 63, pp. 1-32, 1994.

Brosseaud. Y., Jean-Luc Delmore, and R. Hiernaux, “ Use of LPC Wheel-Tracking Rutting Tester to Select Asphalt Pavements Resistant to Rutting,” Transportation Research Record 1384, pp 59-68, Washington D.C., 1993.

Brown, E.R. and S.A. Cross, "A National Study of Rutting in Hot Mix Asphalt (HMA) Pavements,” NCAT Report No. 92-5, National Center for Asphalt Technology, Auburn, AL, 1992.

Brown, E.R., "Draft Proposal for Accelerated Loading Pavement Study,” Auburn University, Auburn, AL, March 1998. 
Brown, E.R., V.P. Erv Dukatz, G. Huber, L. Micheal, J. Scherrocman, and R. Sines, "Performance of coarse graded Mixes at the WesTrack- Premature Rutting ," Final Report prepared for Federal Highway Administration, June 1998.

Brown, E.R., and R.B. Powell, “A General Overview of Research Efforts at the NCAT Pavement Test Track,” Paper 01-149, Proceedings of the Second International Symposium on Maintenance and Rehabilitation of Pavements and Technological Control, Auburn, Alabama, July 29-Aug. 1, 2001

Brown, E.R., P.S. Kandhal, and J. Zhang, “ Performance Testing for Hot Mix Asphalt,” NCAT Report No. 2001-05A, November 2001.

Brown, E.R., A. Cooley, D. Hanson, C. Lynn, , Buzz Powell, Brian Powell, and D. Watson, "NCAT Test Track Design Construction and Performance,” Report, published for National Transportation Symposium, November 13-14 2002, Auburn, Alabama

Brown, S.F., and M.S. Sniath, “The permanent Deformation Characteristics of a Dense Bitumen Macadam subjected to Repeated Loading,” Journal of the Association of Asphalt Paving Technologists, Volume 43, Association of Asphalt Paving Technologists, pp 224-252, 1974.

Brown, S.F., and J.M. Gibb, "Validation Experiments for Permanent deformation Testing of Bituminous Mixtures”, Journal of the Association of Asphalt Paving Technologists, Volume 65, Association of Asphalt Paving Technologists, St Paul, Minnesota, 1996.

Chadbourn, B.A., D.E. Newcomb, and D.H. Timm, “ Measured and Theoretical Comparisons of Traffic loads and Pavement Response Distributions,” Paper presented at the Eighth International Conference on Asphalt Pavements, Seattle, Washington, August 1997.

Chen, D.H., J. Bilyeu, D. Walker, and M. Murphy, "Study of Rut-Depth Measurements," Paper No. 01-0139, Paper submitted for the $80^{\text {th }}$ Annual Meeting, Transportation Research Board, Washington, D.C., 2001

Chen, Dar-Hao, and H. Lin, "Development of an equation to predict permanent deformation,” Paper presented for the TRB Annual Meeting, 2001, Washington D.C.

Christensen, D.W., and R. Bonaquist, "Rut resistance and Volumetric Composition of Asphalt Concrete Mixtures,” Association of Asphalt Paving Technologists, Proceedings of the Technical Sessions, Vol. 74, 2005, pp. 410-439.

Davis, R., “Large Stone Matrix: An Historical Insight,” National Asphalt Paving Association, IS-103, 1988. 
Dawley, C.B., B.L Hogewiede, and K.O. Anderson,"Mitigation of Instability Rutting of Asphalt Concrete Pavement in Leth Bridge,” Association of Asphalt Paving Technologists, Vol 59, pp.481- 508, 1990.

De Beer, M. and C.Fisher, , "Contact Stresses of pneumatic tires measured with the Vechile-Road Surface Pressure Transducer Array (VRSPTA) system for the University of California Berkeley (UCB) and the Nevada Automotive Test Center (NATC)," Vol. 1, Confidential/Contract Research Report CR-97/053, CSIR, Pretoria, South Africa, June 1997.

Epps, J.A., and V.Quintus, "Highway Materials Eengineering, Module V: Asphalt Materials and Paving Mixtures,” Report No: FHWA-HI-90-008, February 1990.

Epps, J.A., C.L.Monismith, S.B.Seeds, S.H.Alavi, S.C.Ashmore, R.B.Leahy, and T.M.Mitchell, "WesTrack Performance- Interim Findings," Journal of the Association of Asphalt Paving Technologists, Vol. 67, St. Paul, MN, 1998, pp. 738-782.

Epps, J.A., R.B. Leahy, T. Mitchell, C. Ashmore, S. Seeds, S. Alavi, and C.L. Monismith, "WesTrack- The Road to Performance-Related Specifications,” Proceedings of the First International Conference on Accelerated Pavement Testing, Reno, Nevada, Oct 18-20, 1999.

Fang, H., “ Layers contribution to the Rutting,” PhD Thesis Submitted to the Faculty, Purdue University, West Lafayette, IN, August, 2000.

FHWA, “Predictive Design Procedures, VESYS,” Users Manual, FHWA Report No. FHWA-RD-77-154, 1978

Finn, F., C. L. Saraf, R. Kulkarni, K. Nair, W. Smith, A. Abdullah, "Development of Pavement Structural Subsystems,” National Cooperative Highway Research Program Report 291, National Research Council, Washington D.C., December 1986

Galal, K.A and T.D. White, “INDOT-APT Facility Experience,” Paper submitted for the International Conference on Accelerated Pavement Testing, Reno, NV, October 1999.

Galal, K. and T.White, "Second Phase Study of Change in In-Service Asphalt (Vol. 1of 2),” Joint Transportation Research Program Report No: FHWA/IN/JTRP-99/07-I, June, 2000

Gardner, M.S., Investigation of Hot Mix Asphalt Mmixtures at Mn/Road, Final Report, Minnesota Department of Transportation, St paul, Minnesota, February 1997.

Habermann, J.A,. "Design Features and a Preliminary Study of Purdue Linear Compactor of the PURWheel Tracking Device,” M.S.Thesis, Purdue University, 1994. 
Haddock, J.E, C.Pan, A.Feng, K.Galal, and T.D.White, "National pooled Fund Study No. 176 - Validation of SHRP Asphalt Mixture Specifications Using Accelerated Testing, ” Interim Report, Indiana Department of Transportation, Division of Research, West Lafayette, IN, December 1998.

Haddock, J.E, and D.R. Ward, "Performance of a Warranted Hot Mix Asphalt Pavement in Indiana, ” Journal of the Association of Asphalt Paving Technologists, Vol. 67, St. Paul, MN, 1998.

Hand, A.J.T., "Relationships between Laboratory Measured HMA material and Mixture Properties and Pavement Performance at the WesTrack," Ph.D. Dissertation, University of Nevada, Reno, NV, August 1998, 805 pp.

Hand, Adam J.T., Lee Gallivan, G. Huber, and Bill Pine, NCAT Test Track Construction Visit, SPR 2199, "Validation of Superpave Mixture Design and Analysis Procedures Using the NCAT Test Track,” Joint Transportation Research Program, West Lafayette, IN, May 31, 2000.

Hand, Adam J.T., J.L Stiady, T.D.White, A.S. Noureldin, and K. Galal, "Gradation Effects on HMA Rutting performance,", Report prepared and submitted for the $80^{\text {th }}$ Annual Transportation Research Board Meeting, November 17, 2000.

Harrigan, E.T., "Significance of As-constructed of HMA air voids to Pavement Perforamcne from an analysis of LTTP Data,” Research Results Digest No. 269, National Cooperative Highway Research Program, September 2002.

Harvey, J., and L. Popescu, “Accelerated Pavement Testing of Rutting Performance of Two Caltrans Overlay Strategies,” Transportation Research Record 1716, Transportation Research Board, National Research Council, Washington, D.C., 2000, pp. 116-125.

Hassan, H.F., and T.D. White, “Locating Drainage Layer for Flexible Pavements,” Final Report, FHWA/IN/JHRP-96/14, Dec., 1996.

Highway Research Board, “The AASHO Road Test- Report 5- Pavement Research,” Publication No. 954, National Research Council, Washington D.C., 1962.

Hofstra, A., and A.J.G. Klomp, "Permanent deformation of the Flexible Pavements Under Simulated Road Traffic Conditions, Preceedings, Third international conference on the structural design of Asphalt Pavements, Vol. I., London, United Kingdom, 1972,pp 613-621 
Hua, J., "Finite Element Modeling and Analysis of Accelerated Pavement Testing Devices and Rutting Phenomenon,” Ph.D. Thesis, Purdue University, West Lafayette, IN University, August 2000.

Huang, H.M., "Analysis of Accelerated Pavement Tests and Finite Element Modeling of Rutting Phenomenon,” Ph.D. Thesis, Purdue University, West Lafayette, IN, August 1995

Huang, H.Y., "Pavement Analysis and Design,” Prentice Hall, Englewood, Cliffs, NJ, 1993.

Huang, B., L.N. Mohammad, and M. Rasoulian, “3-D Numerical Simulation of Asphalt Pavement At Louisiana Accelerated Loading Facility (ALF), CD-ROM print of the paper submitted for the Transportation Research Board Annual Meeting, 2000.

Hugo, F., and A.M. Epps, "Significant Findings from Full-Scale Accelerated Pavement Testing, NCHRP Synthesis 235, Transportation Research Board, Washington D.C., 2004.

Jackson, N. M., and D. C. Baldwin, "Evaluation of Asphalt Pavement Analyzer to Predict the Relative Rutting Susceptibility of HMA in Tennessee," Proceedings of the International Conference on Accelerated Pavement Testing, Paper Number CS6-3, The University of Nevada, Reno, Nevada, October 18-20, 1999, 15 pages.

Kasahara, A., "Wheel Path Distribution of Vehicles on Highway," Proceedings of the International Symposium on Bearing Capacity of Roads and Airfields, Volume I, pp. 413-420, Trondheim, Norway, June 1982.

Kandhal, P.S., and L.A. Cooley, "Evaluation of Permanent Deformation of Asphalt Mixtures using Loaded Wheel Tester,” NCAT Report No. 2002-08

Kandhal, P.S., and R.B. Mallick, "Evaluation of Asphalt Pavement Analyzer for HMA Mixture Design,” NCAT Report No. 1999-04, June 1999.

Kandhal, P.S., and L.A. Cooley,Jr., "Accelerated Laboratory Rutting Tests: Evaluation of the Asphalt Pavement Analyzer," National Co-operative Highway Research Program Report No 508, Washington D.C., 2003.

Kirkner, D.J., W. Shen, M.I. Hammons, and M.D. Smith,"Numerical Simulation of Permanent Deformation in Flexible Pavement Systems Subjected to Moving Loads," Proceedings of the $11^{\text {th }}$ Conference on Engineering Mechanics, American Society of Civil Engineers, Fort Lauderdale, Florida, May 19-22,1996, pp. 430-433.

Kopperman, S., G. Tiller, and M. Tseng, "ELSYM5, Interactive Microcomputer Version, User’s Manual, Report No. FHWA-TS-87-206, 1986. 
Kraus, H., “Creep Analysis,” John Wiley \& Sons, New York, NY, 1980.

Leahy, R.B., E.T. Harrigan, and H.V. Quintus, "Validation of Relationships Between Specification Properties and Performance,” Strategic Highway Research Program, Report No. SHRP-A-409, National Research Council, Washington, D.C., August 1994.

Logan, D.L., “A First Course in the Finite Element Method,” PWS Publishing Company, 1993.

Ljung, E., and J. Sjoberg, “ Non-linear-FE-Analysis of Pavement Deformations in Road Pavements,” Master theis, Chalmers University of Technology, 2000.

Mates, "Premature Rutting Can be Prevented" Technical Paper, No. 19, Michigan Department of Transportation, March 1989, 2 pages.

McGennis, R.B., R.M. Anderson, T.W. Kennedy, and M. Solaimanian, "Background of Superpave Asphalt Mixture Design \& Analysis System,” FHWA Report No. FHWASA-95-003, February 1995.

Metcalf, J.B., “Application of Full-Scale Accelerated Pavement Testing,” NCHRP Synthesis 235, National Academic Press, Washington D.C., 1996.

Mohammad, L.N., B.Huang, F.Roberts and M. Rasoulian, “Accelerated Loading Performance of Crumb Rubber Asphalt Pavements, International Journal of Road Materials and Pavement Design, Vol.1, 2000, pp. 467-493.

Monismith, C.L., J.A. Epps, and F.N. Finn, “Improved Asphalt Mmixture Design,” Journal of the Association of Asphalt Paving Technologists, Vol. 54, pp 347-406, 1985.

Monismith, C.L., K. Inkabi, C.R. Freeme, and D.B.Mclean, “A Subsystem to predict Rutting in Asphalt Concrete Pavement Structures,” Proceedings, Fourth Iinternational Conference on Structural Design of Asphalt Pavements, Vol 1. Ann Arbor, pp. 529-539, 1977.

Monismith, C.L. and A.A. Tayebeli, "Permanent Deformation considerations in Asphalt Pavement Sections," Journal of the Association of Asphalt Paving Technologists, Vol. 57, pp 414-463, 1988.

Moseley, H.L., G.C.Page, J.A. Musselman, G.A. Scholar, and P.B.Upshaw, "Laboratory Mixtures and Binder Rutting Study” Research Report FL/DOT/SMO/03-465, August 2003, Gainesville,FL. 
Mulvaney, R. and B. Worel, “MnROAD Mainline Rutting Forensic Investigation,” Final Report, Minnesota Department of Transportation, Office of Materials and Road Research, St Paul, Minnesota, October 2002.

Newcomb, D.E., G. Engstrom, D.A. Van Deusen, J.A. Siekmeier, and D.H. Timm, "Minnesota Road Research Project: A Five- Year Review of Accomplishments," Proceedings of the First International Conference on Accelerated Pavement Testing, Reno, Nevada, Oct. 18-20, 1999.

Olsson, J., L. Zeng, and N.E. Wiberg, "Finite Element Analysis of Road Rutting," European Congress on Computational Methods in Applied Sciences and Engineering, Barcelona, Spain, September 11-14, 2000, 15 pages.

Pan, C.L.,” Analysis of Bituminous Mixtures Stripping/Rutting Potential,” Ph.D. Thesis, Purdue University, West Lafayette, IN, August 1997.

Perl, Mordechi, J. Uzan, and A. Sides, “ Visco-Elasto-Plastic Constitutive Law for a Bituminous Mixture Under Repeated Loading,” Transportation Research Record 911, National Research Council, Washington, D.C., pp. 20-27, 1983.

Pirabarooban, S., Modeling of Rutting in Asphalt Mixes Using ABAQUS, M. S. Thesis, University of Oklahoma, Norman, 2002.

Powell, R.B., "As-Built Properties of Experimental Sections on the 2000 NCAT Pavement Test Track,” April 2001, NCAT, Auburn, Alabama.

Powell, R.B., "Laboratory Performance Testing for the NCAT Pavement Test Track,” A paper prepared for the $82^{\text {nd }}$ Annual Transportation Research Board Meeting, CD-ROM print, July 2002.

Roberts, F.L., P.S. Kandhal, , R.E. Brown, , D-Y Lee, , T.W. Kennedy, "Hot Mix Aspahlt Matrials,Mixture Design and Construction,” NAPA Education Foundation Incorporated, Lanham, Maryland, $2^{\text {nd }}$ Edition, 1996.

Seibi, A.C., M.G. Sharma, G.A. Ali and W.J. Kenis, "Constitutive Relationships for Asphalt Concrete Under Hhigh Rates of Loading.” Paper offered for presentation and publication at the Transportation Research Board, $80^{\text {th }}$ Annual Meeting, Washington D.C., November 2000.

Sharp, K.G., J.R. Johnson-Clarke, and D.W.Fossey, "A Review of the Australian ALF Program," Proceedings of $1^{\text {st }}$ International Conference on Accelerated Pavement Testing, Reno, NV, October 1999. 
Sivasubramaniam, S. and J.E.Haddock, “Validation of Superpave Mixture Design and Analysis Procedures Using the NCAT Test Track,” Report prepared and submitted for the $82^{\text {nd }}$ Annual Transportation Research Board Meeting, August 2002.

Sivasubramaniam, S., K.A. Galal, A.S. Noureldin, T.D. White, and J.E. Haddock, “ Modleing permanent Deformation Using Laboratory, Proto-type, and In-service Accelerated Pavement Testing," Report prepared and submitted for the $83^{\text {rd }}$ Annual Transportation Research Board Meeting, August 2003.

Sousa, J.B., S.L. Weissman, J. L. Sackman, and C.L. Monismith, "Nonlinear Elastic Viscous with Damage Model to Predict Permanent Deformation of Asphalt Concrete Mixes,” Transportation Research Record 1384, National Research Council, Washington D.C., 1993.

Stewart, K.D., W.S. Mogawer, and P. Romero, "Validation of Asphalt Binder and Mixture Tests that Measure Rutting Susceptibility Using Accelerated Loading Facility," FHWA Report No. FHWA-RD-99-204, November 2000.

Stiady, J.L., T.D. White, and C.R. Reck, “Rut 3.3”, A computer Program to Process APT Data, Users Manual, Purdue University, West Lafayette, IN, June 1998.

Stiady, J.L., "Validation of SHRP Asphalt Mixture Specifications Using Accelerated Testing,” Ph.D. Thesis, Purdue University, West Lafayette, IN, May 2000.

Stiady, J.L., A.J.T. Hand, A.S. Noureldin, K. Galal, J. Hua, and T.D. White, "Validation of SHRP Asphalt Mixture Specifications Using Accelerated Testing,” Final Report, FHWA National Pooled Fund Study No. 176, Report No: FHWA/IN/JTRP2003/02,February 2003.

Superpave Mixture Design, Training course Manual, 1996.

Uge, P. and P.J. Van de Loo, "Permanent Deformation of the Asphalt Mmixes," Shell Laboratoriam, Amsterdam, November 1974.

White, T.D., "Research Proposal for Validation of Superpave Mixture Design and Analysis Procedures Using the NCAT Test Track,” Purdue University, West Lafayette, IN, 1998.

White, T.D., J. Hua, and K.A. Galal, “Analysis of Accelerated Pavement Tests,” Paper submitted for the International Conference on Accelerated Pavement Testing, Reno, NV, October 1999. 
White, T.D., J.E. Haddock, A.J.T. Hand, and H. Fang, "Contributions to Pavement Structural Pavement Layers to Rutting of Hot Mix Asphalt Pavements," NCHRP Report 468, National Academic Press, Washington D.C., 2002.

Williams, R.C., P. Romero, and K.D. Stewart, "Comparison of Superpave Shear Tester Results to WesTrack Performance, Proceeding of the International conference of the Accelerated Pavement Testing, Reno, NV, October 1999.

Witczak, M.W., K. Kaloush, T. Pellinen, El-Basyouny, and H. Von Quintus, , "Simple Performance Test for Superpave Mix Design,” NCHRP Report 465, National Academic Press, Washington D.C.2002.

Witczak, M.W., S.H. Cardoso, and K. Kaloush, “ Triaxial Strength Test,” National Co operative Highway Research Program Project 9-19, Task C, Team Report SPTALF2(D), ALF experiment, Arizona State University, Tempe, AZ, 2000.

Wu, Z., M. Hossain, and A.J. Gisi, "Performance of Superpave mixtures Under Accelerated Load Testing”, Paper presented for the 79 th Annual Meeting of the Transportation Board, Preprint CD Rom, Washington D.C., 2000.

Yandell, W.O., "The Prediction of the Behavior of Elastoplastic Roads During Repeated Rolling Using Mechano-Lattice Analog,” Highway Research Record 374, Highway Research Board, National Research Council, Washington D.C., 1971, pp. 29-41.

Yandell, W.O., "Mechano-Lattice Prediction of Pavement Performance," Proceedings, CAPSA, Capetown, South Africa, March 12-16, 1984, pp. 201-215.

Yandell, W.O., and G. Behzadi, "Rutting Prediction of Twelve Accelerated Loading Facility (ALF) Trials,” Proceeding of the International Conference on Accelerated Pavement Testing, Reno, Nevada, October 1999.

Zaghloul, S.M., and T.D. White, " Use of Three dimensional Finite Element Program for Flexible Pavement,” Transportation Research Record, No.1388, TRB, National Research Council, Washington D.C., 1993, pp. 60-69

Zerfas, W.J. , 2002 Superpave Report, Mn/Dot office of Materials and Road Research, May 19, 2003.

Zhang, J., L.A. Cooley, and P.S. Kandhal "Comparison of Fundamental and Simulative Test Methods for Evaluating Permanent Deformation of Hot Mix Asphalt," NCAT Report No. 02-07, October 2002. 
www.pavetrack.com, Official website of the NCAT Test Track.

www.engg.ksu.edu/CEDEPT/research/resatlfaci_bd.htm, Kansas ALF webpage. 